\title{
Catalytic Asymmetric Hydrogenation of 3-Ethoxycarbonyl Quinolin-2-ones and Coumarins
} Qian-Kun Zhao ${ }^{a}$, Xiong Wu ${ }^{a}$, Fan Yang ${ }^{a}$, Pu-Cha Yan ${ }^{b, *}$, Jian-Hua Xie ${ }^{a, *}$, Qi-Lin hou $^{a}$

\author{
${ }^{a}$ State Key Laboratory and Institute of Elemento-organic Chemistry, College of Chemistry, Nankai \\ University, Tianjin 300071, China \\ ${ }^{b}$ Raybow (Hangzhou) Pharmaceutical Science \& Technology CO., Ltd., Hangzhou 310018, China
}

\section{Contents}

General: . $\mathrm{S1}$

(A) Preparation of 3-Ethoxycarbonyl Quinolin-2-ones and Coumarins.............................S2

(B) Asymmetric Hydrogenation of 3-Ethoxycarbonyl Quinolin-2-ones and Coumarins............S11

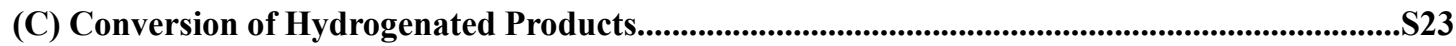

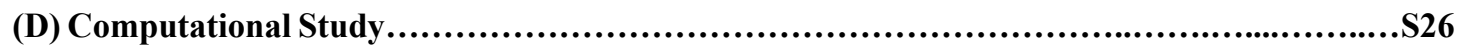

(E) NMR Spectra of New Compounds.........................................................S30

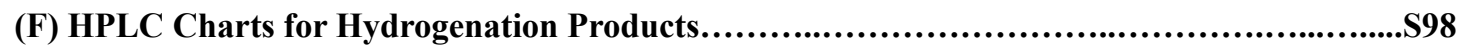

(G) Cartesian Coordinates and Energies of the Optimized structures.............................S129

\section{General:}

All reactions and manipulations which are sensitive to moisture or air were performed under an inert atmosphere of nitrogen. All chemicals were purchased from J\&K, Acros and Aldrich and were used as received. Hydrogen gas (99.999\%) was purchased from Boc Gas Inc., Tianjin. Anhydrous THF was distilled from sodium benzophenone ketyl. Anhydrous $\mathrm{CH}_{2} \mathrm{Cl}_{2}$ and $\mathrm{Et}_{3} \mathrm{~N}$ were freshly distilled from calcium hydride. Anhydrous EtOH was freshly distilled from magnesium. Melting points were measured on a RY-I apparatus and uncorrected. ${ }^{1} \mathrm{H}$ NMR spectra were recorded at $400 \mathrm{MHz}$ on Bruker AV 400 spectrometer. ${ }^{13} \mathrm{C}$ NMR spectra were recorded at $100 \mathrm{MHz}$ on Bruker AV 400 spectrometer. NMR spectra were recorded in deuterated chloroform $\left(\mathrm{CDCl}_{3}\right)$ as a solvent, with residual chloroform $(\delta: 7.26$ ppm. for ${ }^{1} \mathrm{H}$ NMR and $\delta: 77.00 \mathrm{ppm}$. for ${ }^{13} \mathrm{C}$ NMR) or tetramethylsilane (TMS, $\delta: 0.00 \mathrm{ppm}$. for ${ }^{1} \mathrm{H}$ NMR) taken as the inert standard, and were reported in ppm. Abbreviations for signal coupling are as follows: s, singlet; d, doublet; $t$, triplet; q, quartet; m, multiplet. Coupling constants were taken from the spectra directly and are uncorrected. Optical rotations were determined using a Perkin Elmer 341 polarimeter. Highresolution mass spectra (HRMS) were acquired using Varian 7.0T FTMS or Agilent 6520 Q-TOF LC/MS with electrospray ionization (ESI) source. HPLC analyses were performed using Hewlett Packard Model HP1100 instruments with Chiralcel AD-H, AS-H, OD-H, OJ-H and OD-3 column. 


\section{(A) Preparation of 3-Ethoxycarbonyl Quinolin-2-ones and Coumarins}

\section{Preparation of 4-substituted 3-ethoxycarbonyl Quinolin-2-ones 2}

4-Substituted 3-ethoxycarbonyl quinolin-2-ones $\mathbf{2 a - m}$ were conveniently synthesized from the known starting material $o$-aminoaryl ketone according to the known literature ${ }^{1}$ followed by $N$-alkylation. ${ }^{2}$

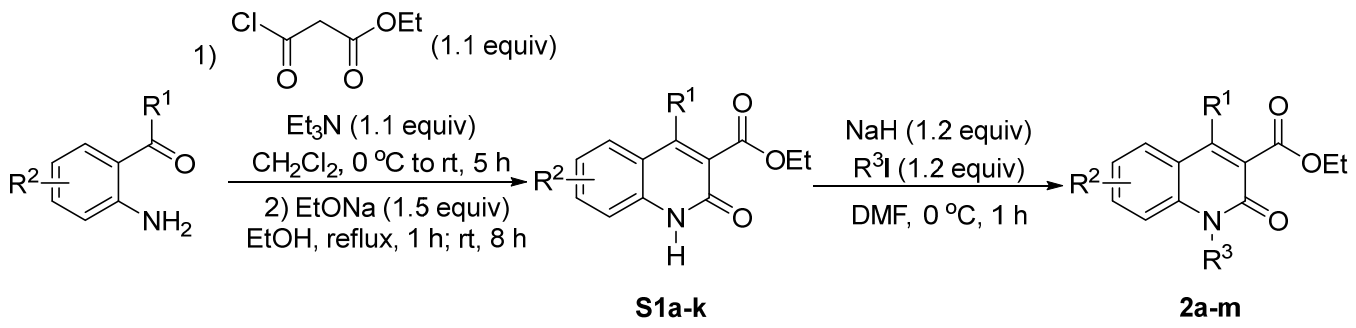

Preparation of ethyl 4-substituted-2-oxo-1,2-dihydroquinoline-3-carboxylate S1a-k

General procedure: ${ }^{1}$ To a solution of $o$-aminoaryl ketone $(5.0 \mathrm{mmol})$ and $\mathrm{Et}_{3} \mathrm{~N}(0.8 \mathrm{~mL}, 5.5 \mathrm{mmol})$ in $\mathrm{CH}_{2} \mathrm{Cl}_{2}(10 \mathrm{~mL})$ was added ethyl malonyl chloride $(0.7 \mathrm{~mL}, 5.5 \mathrm{mmol})$ dropwise with stirring at $0{ }^{\circ} \mathrm{C}$. The resulting reaction mixture was then stirred at room temperature for $5 \mathrm{~h}$ and diluted with water (10 $\mathrm{mL}$ ). The organic layer was separated, dried over anhydrous $\mathrm{Na}_{2} \mathrm{SO}_{4}$, and concentrated in vacuo. The residue was then redissolved with a solution of EtONa $(510 \mathrm{mg}, 7.5 \mathrm{mmol})$ in absolute EtOH $(15 \mathrm{~mL})$. The resulting solution was then heated to reflux in an oil bath for $1 \mathrm{~h}$ and stirred at room temperature for $8 \mathrm{~h}$. The reaction mixture was diluted with water $(10 \mathrm{~mL})$ and acidified with $2 \mathrm{~N} \mathrm{HCl}(10 \mathrm{~mL})$ to $\mathrm{pH}<4$. The resulting precipitate was filtered, washed with cold water $(3 \times 20 \mathrm{~mL})$, and then dried to afford the corresponding product as a white or light yellow solid.

\section{Ethyl 4-methyl-2-oxo-1,2-dihydroquinoline-3-carboxylate (S1a)}<smiles>CCOC(=O)c1c(C)c2ccccc2[nH]c1=O</smiles>

Prepared according to the general procedure from 1-(2-aminophenyl)ethan-1-one (675 mg, $5.0 \mathrm{mmol}$ ), to provide the title compound as white solid, m.p. $245-246^{\circ} \mathrm{C}$, $1.00 \mathrm{~g}, 86 \%$ yield. ${ }^{1} \mathrm{H}$ NMR $\left(400 \mathrm{MHz}, \mathrm{CDCl}_{3}\right) \delta: 11.71(\mathrm{~s}, 1 \mathrm{H}), 7.74(\mathrm{dd}, J=8.0$, $1.2 \mathrm{~Hz}, 1 \mathrm{H}), 7.54$ (ddd, $J=8.4,7.2,1.6 \mathrm{~Hz}, 1 \mathrm{H}), 7.36$ (dd, $J=8.0,1.2 \mathrm{~Hz}, 1 \mathrm{H}), 7.30$ $7.21(\mathrm{~m}, 2 \mathrm{H}), 4.50(\mathrm{q}, J=7.2 \mathrm{~Hz}, 2 \mathrm{H}), 2.52(\mathrm{~s}, 3 \mathrm{H}), 1.45(\mathrm{t}, J=7.2 \mathrm{~Hz}, 3 \mathrm{H})$ (the spectra are in accordance with those of the compound reported in the literature).

\section{Ethyl 4-ethyl-2-oxo-1,2-dihydroquinoline-3-carboxylate (S1b)}<smiles>CCOC(=O)c1c(CC)c2ccccc2[nH]c1=O</smiles>

Prepared according to the general procedure from 1-(2-aminophenyl)propan-1-one (745 mg, $5.0 \mathrm{mmol}$ ), to provide the title compound as white solid, m.p. $174-175^{\circ} \mathrm{C}$, $1.10 \mathrm{~g}, 90 \%$ yield. ${ }^{1} \mathrm{H}$ NMR (400 MHz, $\left.\mathrm{CDCl}_{3}\right) \delta: 12.93(\mathrm{~s}, 1 \mathrm{H}), 7.74(\mathrm{~d}, J=8.1 \mathrm{~Hz}$, $1 \mathrm{H}), 7.63-7.41(\mathrm{~m}, 2 \mathrm{H}), 7.32-7.16(\mathrm{~m}, 1 \mathrm{H}), 4.51$ (q, $J=7.1 \mathrm{~Hz}, 2 \mathrm{H}), 2.88$ (q, $J=7.6$ $\mathrm{Hz}, 2 \mathrm{H}), 1.45(\mathrm{t}, J=7.1 \mathrm{~Hz}, 3 \mathrm{H}), 1.34(\mathrm{t}, J=7.5 \mathrm{~Hz}, 3 \mathrm{H}) ;{ }^{13} \mathrm{C} \mathrm{NMR}\left(101 \mathrm{MHz}, \mathrm{CDCl}_{3}\right) \delta: 166.6,161.4$, $151.7,138.5,131.2,125.4,124.8,122.9,118.3,117.4,61.6,23.8,14.5,14.3$. HRMS (ESI) m/z: $[\mathrm{M}+\mathrm{Na}]^{+}$ calcd for $\mathrm{C}_{14} \mathrm{H}_{15} \mathrm{NO}_{3} \mathrm{Na} 268.0944$; Found 268.0948.

\section{Ethyl 2-oxo-4-propyl-1,2-dihydroquinoline-3-carboxylate (S1c)}

Prepared according to the general procedure from 1-(2-aminophenyl)butan-1-one (815 mg, $5.0 \mathrm{mmol})$, 
<smiles>CCOC(=O)c1c(C(C)C)c2ccccc2[nH]c1=O</smiles>

to provide the title compound as white solid, m.p. $157-158{ }^{\circ} \mathrm{C}, 1.20 \mathrm{~g}, 93 \%$ yield. ${ }^{1} \mathrm{H}$ NMR $\left(400 \mathrm{MHz}, \mathrm{CDCl}_{3}\right) \delta: 12.83(\mathrm{~s}, 1 \mathrm{H}), 7.72(\mathrm{~d}, J=8.1 \mathrm{~Hz}, 1 \mathrm{H}), 7.62-7.42(\mathrm{~m}$, 2H), 7.24 (t, $J=7.6 \mathrm{~Hz}, 1 \mathrm{H}), 4.51$ (q, $J=7.1 \mathrm{~Hz}, 2 \mathrm{H}), 2.83(\mathrm{t}, J=8.1 \mathrm{~Hz}, 2 \mathrm{H}), 1.75$ (q, $J=7.8 \mathrm{~Hz}, 2 \mathrm{H}), 1.45(\mathrm{t}, J=7.1 \mathrm{~Hz}, 3 \mathrm{H}), 1.07(\mathrm{t}, J=7.3 \mathrm{~Hz}, 3 \mathrm{H}) ;{ }^{13} \mathrm{C}$ NMR $(101$ $\left.\mathrm{MHz}, \mathrm{CDCl}_{3}\right) \delta: 166.7,161.4,150.4,138.4,131.1,125.8,124.9,122.8,118.7,117.3,61.6,32.5,23.5$, 14.5, 14.3. HRMS (ESI) m/z: [M+Na $]^{+}$calcd for $\mathrm{C}_{15} \mathrm{H}_{17} \mathrm{NO}_{3} \mathrm{Na} 282.1101$; Found 282.1105.

\section{Ethyl 4-isobutyl-2-oxo-1,2-dihydroquinoline-3-carboxylate (S1d)}<smiles>CCOC(=O)c1c(CC)c2ccccc2[nH]c1=O</smiles>

Prepared according to the general procedure from 1-(2-aminophenyl)-3methylbutan-1-one $(885 \mathrm{mg}, 5.0 \mathrm{mmol})$, to provide the title compound as white solid, m.p. $183-184{ }^{\circ} \mathrm{C}, 1.13 \mathrm{~g}, 83 \%$ yield. ${ }^{1} \mathrm{H}$ NMR $\left(400 \mathrm{MHz}, \mathrm{CDCl}_{3}\right) \delta: 12.90$ (s, $\left.1 \mathrm{H}\right)$, $7.74(\mathrm{~d}, J=8.0 \mathrm{~Hz}, 1 \mathrm{H}), 7.52(\mathrm{t}, J=7.6 \mathrm{~Hz}, 1 \mathrm{H}), 7.47(\mathrm{~d}, J=7.6 \mathrm{~Hz}, 1 \mathrm{H}), 7.29-7.18$ (m, 1H), 4.50 (q, J=7.2 Hz, 2H), $2.81(\mathrm{~d}, J=7.6 \mathrm{~Hz}, 2 \mathrm{H}), 2.20-2.04(\mathrm{~m}, 1 \mathrm{H}), 1.45$ (t, J=7.2 Hz, 3H), $0.99(\mathrm{~d}, J=6.4 \mathrm{~Hz}, 6 \mathrm{H}) ;{ }^{13} \mathrm{C}$ NMR $\left(101 \mathrm{MHz} \mathrm{CDCl}_{3}\right) \delta: 166.8,161.4,149.6,138.3,131.1,126.6,125.4$, 122.7, 119.1, 117.3, 61.6, 38.8, 29.4, 22.8, 14.3. HRMS (ESI) m/z: $[\mathrm{M}+\mathrm{H}]^{+}$calcd for $\mathrm{C}_{16} \mathrm{H}_{20} \mathrm{NO}_{3}$ 274.1438; Found 274.1439.

\section{Ethyl 4-benzyl-2-oxo-1,2-dihydroquinoline-3-carboxylate (S1e $)^{3}$}<smiles>CCOC(=O)c1c(Cc2ccccc2)c2ccccc2[nH]c1=O</smiles>
Prepared according to the general procedure from 1-(2-aminophenyl)-2phenylethan-1-one (1.06 g, $5.0 \mathrm{mmol})$, to provide the title compound as white solid, m.p. $206-207{ }^{\circ} \mathrm{C}, 0.90 \mathrm{~g}, 59 \%$ yield. ${ }^{1} \mathrm{H}$ NMR $\left(400 \mathrm{MHz}, \mathrm{CDCl}_{3}\right) \delta: 12.60(\mathrm{~s}, 1 \mathrm{H})$, $7.65(\mathrm{~d}, J=8.4 \mathrm{~Hz}, 1 \mathrm{H}), 7.51-7.39(\mathrm{~m}, 2 \mathrm{H}), 7.31-7.24(\mathrm{~m}, 4 \mathrm{H}), 7.24-7.16(\mathrm{~m}, 1 \mathrm{H})$, 7.12 (ddd, $J=8.4,6.8,1.6 \mathrm{~Hz}, 1 \mathrm{H}), 4.44$ (q, $J=7.2 \mathrm{~Hz}, 2 \mathrm{H}), 4.27$ (s, 2H), 1.32 (t, $J=7.2 \mathrm{~Hz}, 3 \mathrm{H})$ (the spectra are in accordance with those of the compound reported in the literature).

\section{Ethyl 2-oxo-4-phenethyl-1,2-dihydroquinoline-3-carboxylate (S1f)}<smiles>CCOC(=O)c1c(CCc2ccccc2)c2ccccc2[nH]c1=O</smiles>
Prepared according to the general procedure from 1-(2-aminophenyl)-3phenylpropan-1-one $(1.12 \mathrm{~g}, 5.0 \mathrm{mmol})$, to provide the title compound as light yellow solid, m.p. $186-187{ }^{\circ} \mathrm{C}, 0.79 \mathrm{~g}, 49 \%$ yield. ${ }^{1} \mathrm{H}$ NMR $\left(400 \mathrm{MHz}, \mathrm{CDCl}_{3}\right) \delta$ : $13.08(\mathrm{~s}, 1 \mathrm{H}), 7.78(\mathrm{~d}, J=8.2 \mathrm{~Hz}, 1 \mathrm{H}), 7.56-7.49$ (m, 2H), 7.36-7.19 (m, 6H), 4.52 (q, $J=7.1 \mathrm{~Hz}, 2 \mathrm{H}), 3.27-3.11(\mathrm{~m}, 2 \mathrm{H}), 3.06-2.91(\mathrm{~m}, 2 \mathrm{H}), 1.45(\mathrm{t}, J=7.2 \mathrm{~Hz}, 3 \mathrm{H}) ;{ }^{13} \mathrm{C} \mathrm{NMR}(101 \mathrm{MHz}$, $\left.\mathrm{CDCl}_{3}\right) \delta: 166.6,161.5,149.5,140.8,138.6,131.4,128.7,128.2,126.5,126.0,124.8,123.1,118.5,117.5$, 61.8, 36.2, 32.6, 14.4. HRMS (ESI) m/z: $[\mathrm{M}+\mathrm{H}]^{+}$calcd for $\mathrm{C}_{20} \mathrm{H}_{20} \mathrm{NO}_{3}$ 322.1438; Found 322.1439.

\section{Ethyl 2-oxo-4-pentyl-1,2-dihydroquinoline-3-carboxylate (S1g)}<smiles>CCOC(=O)c1c(C=O)c2ccccc2[nH]c1=O</smiles>

Prepared according to the general procedure from 1-(2-aminophenyl)hexan-1-one (955 mg, $5.0 \mathrm{mmol})$, to provide the title compound as white solid, m.p. $138-139^{\circ} \mathrm{C}$, $1.21 \mathrm{~g}, 84 \%$ yield. ${ }^{1} \mathrm{H}$ NMR $\left(400 \mathrm{MHz}, \mathrm{CDCl}_{3}\right) \delta: 12.90(\mathrm{~s}, 1 \mathrm{H}), 7.72(\mathrm{~d}, J=8.1 \mathrm{~Hz}$, $1 \mathrm{H}), 7.56-7.43(\mathrm{~m}, 2 \mathrm{H}), 7.25(\mathrm{t}, J=7.6 \mathrm{~Hz}, 1 \mathrm{H}), 4.51(\mathrm{q}, J=7.1 \mathrm{~Hz}, 2 \mathrm{H}), 2.92-2.77$ (m, 2H), 1.78-1.65 (m, 2H), $1.41(\mathrm{~d}, J=51.1 \mathrm{~Hz}, 7 \mathrm{H}), 0.92(\mathrm{t}, J=7.1 \mathrm{~Hz}, 3 \mathrm{H}) ;{ }^{13} \mathrm{C}$ NMR $(101 \mathrm{MHz}$, $\left.\mathrm{CDCl}_{3}\right) \delta: 166.7,161.4,150.6,138.5,131.1,125.7,124.9,122.8,118.7,117.3,61.6,32.2,30.5,29.9$, 22.4, 14.3, 14.0. HRMS (ESI) m/z: $[\mathrm{M}+\mathrm{H}]^{+}$calcd for $\mathrm{C}_{17} \mathrm{H}_{22} \mathrm{NO}_{3} 288.1594$; Found 288.1595.

Ethyl 4-cyclopropyl-2-oxo-1,2-dihydroquinoline-3-carboxylate (S1h) 
<smiles>CCOC(=O)c1c(C2CC2)c2ccccc2[nH]c1=O</smiles>

Prepared according to the general procedure from (2aminophenyl)(cyclopropyl)methanone $(805 \mathrm{mg}, 5.0 \mathrm{mmol})$, to provide the title compound as white solid, m.p. $175-176{ }^{\circ} \mathrm{C}, 1.05 \mathrm{~g}, 82 \%$ yield. ${ }^{1} \mathrm{H}$ NMR (400 MHz, $\left.\mathrm{CDCl}_{3}\right) \delta: 12.87(\mathrm{~s}, 1 \mathrm{H}), 8.17(\mathrm{~d}, J=8.1 \mathrm{~Hz}, 1 \mathrm{H}), 7.51(\mathrm{t}, J=8.2 \mathrm{~Hz}, 1 \mathrm{H}), 7.44(\mathrm{~d}, J$ $=8.1 \mathrm{~Hz}, 1 \mathrm{H}), 7.25(\mathrm{q}, J=8.2,7.5 \mathrm{~Hz}, 1 \mathrm{H}), 4.51(\mathrm{q}, J=7.2 \mathrm{~Hz}, 2 \mathrm{H}), 2.20-2.05(\mathrm{~m}, 1 \mathrm{H}), 1.46(\mathrm{t}, J=7.1$ $\mathrm{Hz}, 3 \mathrm{H}), 1.18-1.05$ (m, 2H), 0.86-0.72 (m, 2H); ${ }^{13} \mathrm{C}$ NMR (101 MHz, $\left.\mathrm{CDCl}_{3}\right) \delta: 166.5,161.6,149.9$, 138.0, 131.1, 127.2, 126.0, 122.7, 120.4, 116.9, 61.6, 14.2, 12.4, 6.6. HRMS (ESI) m/z: $[\mathrm{M}+\mathrm{H}]^{+}$calcd for $\mathrm{C}_{15} \mathrm{H}_{16} \mathrm{NO}_{3} 258.1125$; Found 258.1125.

\section{Ethyl 2-oxo-4-phenyl-1,2-dihydroquinoline-3-carboxylate (S1i) ${ }^{4}$}<smiles>CCOC(=O)c1c(-c2ccccc2)c2ccccc2[nH]c1=O</smiles>

Prepared according to the general procedure from (2aminophenyl)(phenyl)methanone $(985 \mathrm{mg}, 5.0 \mathrm{mmol})$, to provide the title compound as white solid, m.p. $174-175^{\circ} \mathrm{C}, 1.32 \mathrm{~g}, 90 \%$ yield. ${ }^{1} \mathrm{H}$ NMR $\left(400 \mathrm{MHz}, \mathrm{CDCl}_{3}\right) \delta$ : $12.63(\mathrm{~s}, 1 \mathrm{H}), 7.62-7.44(\mathrm{~m}, 5 \mathrm{H}), 7.43-7.35(\mathrm{~m}, 2 \mathrm{H}), 7.30$ (d, $J=8.0 \mathrm{~Hz}, 1 \mathrm{H}), 7.14$ $(\mathrm{t}, J=7.4 \mathrm{~Hz}, 1 \mathrm{H}), 4.13(\mathrm{q}, J=7.2 \mathrm{~Hz}, 2 \mathrm{H}), 0.97(\mathrm{t}, J=7.2 \mathrm{~Hz}, 3 \mathrm{H})$ (the spectra are in accordance with those of the compound reported in the literature).

\section{Ethyl 6-bromo-4-methyl-2-oxo-1,2-dihydroquinoline-3-carboxylate (S1j) ${ }^{5}$}<smiles>CCOC(=O)c1c(C)c2cc(Br)ccc2[nH]c1=O</smiles>

Prepared according to the general procedure from 1-(2-amino-5bromophenyl)ethan-1-one $(1.06 \mathrm{~g}, 5.0 \mathrm{mmol})$, to provide the title compound as white solid, m.p. $225-226{ }^{\circ} \mathrm{C}, 1.34 \mathrm{~g}, 87 \%$ yield. ${ }^{1} \mathrm{H}$ NMR $\left(400 \mathrm{MHz}, \mathrm{CDCl}_{3}\right)$ $\delta: 12.64(\mathrm{~s}, 1 \mathrm{H}), 7.85(\mathrm{~d}, J=2.0 \mathrm{~Hz}, 1 \mathrm{H}), 7.62(\mathrm{dd}, J=8.8,2.0 \mathrm{~Hz}, 1 \mathrm{H}), 7.31(\mathrm{~d}$, $J=8.8 \mathrm{~Hz}, 1 \mathrm{H}), 4.50(\mathrm{q}, J=7.2 \mathrm{~Hz}, 2 \mathrm{H}), 2.48(\mathrm{~s}, 3 \mathrm{H}), 1.45(\mathrm{t}, J=7.2 \mathrm{~Hz}, 3 \mathrm{H})$ (the spectra are in accordance with those of the compound reported in the literature).

\section{Ethyl 6-methoxy-4-methyl-2-oxo-1,2-dihydroquinoline-3-carboxylate (S1k)}<smiles>CCOC(=O)c1c(C)c2cc(OC)ccc2[nH]c1=O</smiles>

Prepared according to the general procedure from 1-(2-amino-5methoxyphenyl)ethan-1-one (825 $\mathrm{mg}, 5.0 \mathrm{mmol})$, to provide the title compound as white solid, m.p. $190-191{ }^{\circ} \mathrm{C}, 1.03 \mathrm{~g}, 79 \%$ yield. ${ }^{1} \mathrm{H}$ NMR (400 $\left.\mathrm{MHz} \mathrm{CDCl}_{3}\right) \delta: 12.85(\mathrm{~s}, 1 \mathrm{H}), 7.39(\mathrm{~d}, J=8.8 \mathrm{~Hz}, 1 \mathrm{H}), 7.18(\mathrm{dd}, J=9.0,2.6$ $\mathrm{Hz}, 1 \mathrm{H}), 7.09$ (d, $J=2.8 \mathrm{~Hz}, 1 \mathrm{H}), 4.51$ (q, $J=7.2 \mathrm{~Hz}, 2 \mathrm{H}), 3.87$ (s, 3H), 2.49 $(\mathrm{s}, 3 \mathrm{H}), 1.45$ (t, $J=7.2 \mathrm{~Hz}, 3 \mathrm{H}) ;{ }^{13} \mathrm{C} \mathrm{NMR}\left(101 \mathrm{MHz}, \mathrm{CDCl}_{3}\right) \delta: 166.8,160.7,155.3,145.4,132.6,126.5$, $120.4,120.3,118.3,106.6,61.7,55.7,16.4,14.3$. HRMS (ESI) m/z: $[\mathrm{M}+\mathrm{H}]^{+}$calcd for $\mathrm{C}_{14} \mathrm{H}_{16} \mathrm{NO}_{4}$ 262.1074; Found 262.1075.

\section{Preparation of ethyl 4-substituted 1-methyl-2-oxo-1,2-dihydroquinoline-3-carboxylate 2a-m}

General procedure: ${ }^{2}$ To a stirring solution of S1a-k $(3.0 \mathrm{mmol})$ in a cold DMF $(5 \mathrm{~mL}), 60 \% \mathrm{NaH}(144$ $\mathrm{mg}, 3.6 \mathrm{mmol}$ ) was added slowly at $0{ }^{\circ} \mathrm{C}$ for $30 \mathrm{~min}$. The resulting reaction mixture was then added alkyl iodide $(3.6 \mathrm{mmol})$ at $0{ }^{\circ} \mathrm{C}$ and continued to stir at the same temperature for $30 \mathrm{~min}$. The reaction mixture was quenched with saturated $\mathrm{NH}_{4} \mathrm{Cl}$ solution $(10 \mathrm{~mL})$, extracted with ethyl acetate $(3 \times 20 \mathrm{~mL})$. The combined extracts were washed with brine, dried over $\mathrm{MgSO}_{4}$, and concentrated in vacuo. The residue was purified by chromatography on silica gel with petroleum ether/ethyl acetate (5:1 to $2: 1)$ as eluent to afford the corresponding product $\mathbf{2} \mathbf{a}-\mathbf{m}$ as a colorless oil or a white solid. 


\section{Ethyl 1,4-dimethyl-2-oxo-1,2-dihydroquinoline-3-carboxylate (2a) ${ }^{6}$}

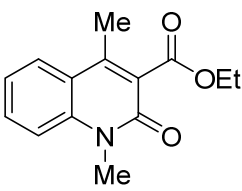

Prepared according to the general procedure from S1a (693 mg, $3.0 \mathrm{mmol}), \mathrm{MeI}$ (511 $\mathrm{mg}, 3.6 \mathrm{mmol}$ ) to provide the title compound as white solid (eluent: petroleum ether/ethyl acetate $=5: 1$ to $2: 1$ ), m.p. $94-95{ }^{\circ} \mathrm{C}, 0.68 \mathrm{~g}, 93 \%$ yield, $R_{\mathrm{f}}=0.7$ (petroleum ether/ethyl acetate $=2: 1) .{ }^{1} \mathrm{H}$ NMR $\left(400 \mathrm{MHz}, \mathrm{CDCl}_{3}\right) \delta: 7.79(\mathrm{dd}, J=$ 8.0, 1.6 Hz, 1H), 7.62 (ddd, $J=8.6,7.2,1.4 \mathrm{~Hz}, 1 \mathrm{H}), 7.39$ (d, $J=8.8 \mathrm{~Hz}, 1 \mathrm{H}), 7.34-7.27$ (m, 1H), 4.46 (q, $J=7.2 \mathrm{~Hz}, 2 \mathrm{H}), 3.73(\mathrm{~s}, 3 \mathrm{H}), 2.48(\mathrm{~s}, 3 \mathrm{H}), 1.42(\mathrm{t}, J=7.2 \mathrm{~Hz}, 3 \mathrm{H})$ (the spectra are in accordance with those of the compound reported in the literature).

\section{Ethyl 4-ethyl-1-methyl-2-oxo-1,2-dihydroquinoline-3-carboxylate (2b)}<smiles>CCOC(=O)c1c(CC)c2ccccc2n([N+](=O)[O-])c1=O</smiles>
Prepared according to the general procedure from S1b ( $735 \mathrm{mg}, 3.0 \mathrm{mmol})$, MeI ( 511 $\mathrm{mg}, 3.6 \mathrm{mmol}$ ) to provide the title compound as colorless oil (eluent: petroleum ether/ethyl acetate $=5: 1$ to $2: 1$ ), $0.70 \mathrm{~g}, 90 \%$ yield, $R_{\mathrm{f}}=0.4$ (petroleum ether/ethyl acetate $=5: 1) .{ }^{1} \mathrm{H}$ NMR $\left(400 \mathrm{MHz}, \mathrm{CDCl}_{3}\right) \delta: 7.78(\mathrm{~d}, J=8.0 \mathrm{~Hz}, 1 \mathrm{H}), 7.58(\mathrm{t}, J=$ $7.8 \mathrm{~Hz}, 1 \mathrm{H}), 7.37$ (d, $J=8.5 \mathrm{~Hz}, 1 \mathrm{H}), 7.28(\mathrm{t}, J=7.6 \mathrm{~Hz}, 1 \mathrm{H}), 4.46$ (q, $J=7.1 \mathrm{~Hz}, 2 \mathrm{H}), 3.69$ (s, 3H), 2.83 $(\mathrm{q}, J=7.5 \mathrm{~Hz}, 2 \mathrm{H}), 1.41(\mathrm{t}, J=7.1 \mathrm{~Hz}, 3 \mathrm{H}), 1.32(\mathrm{t}, J=7.6 \mathrm{~Hz}, 3 \mathrm{H}) ;{ }^{13} \mathrm{C}$ NMR $\left(101 \mathrm{MHz}, \mathrm{CDCl}_{3}\right) \delta$ : 166.6, 159.2, 148.7, 139.8, 131.3, 125.9, 125.7, 122.4, 119.0, 114.7, 61.6, 29.4, 23.6, 14.4, 14.2. HRMS (ESI) $\mathrm{m} / \mathrm{z}:[\mathrm{M}+\mathrm{H}]^{+}$calcd for $\mathrm{C}_{15} \mathrm{H}_{18} \mathrm{NO}_{3} 260.1281$; Found 260.1288 .

\section{Ethyl 1-methyl-2-oxo-4-propyl-1,2-dihydroquinoline-3-carboxylate (2c)}

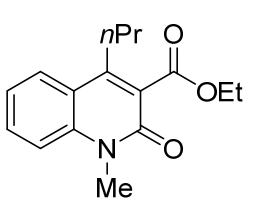

Prepared according to the general procedure from S1c ( $777 \mathrm{mg}, 3.0 \mathrm{mmol})$, MeI (511 $\mathrm{mg}, 3.6 \mathrm{mmol}$ ) to provide the title compound as white solid (eluent: petroleum ether/ethyl acetate $=5: 1$ to $2: 1)$, m.p. $77-78{ }^{\circ} \mathrm{C}, 0.69 \mathrm{~g}, 84 \%$ yield, $R_{\mathrm{f}}=0.4$ (petroleum ether/ethyl acetate $=5: 1) .{ }^{1} \mathrm{H}$ NMR $\left(400 \mathrm{MHz}, \mathrm{CDCl}_{3}\right) \delta: 7.78(\mathrm{~d}, J=8.1$ $\mathrm{Hz}, 1 \mathrm{H}), 7.60$ (t, $J=7.8 \mathrm{~Hz}, 1 \mathrm{H}), 7.38(\mathrm{~d}, J=8.5 \mathrm{~Hz}, 1 \mathrm{H}), 7.28(\mathrm{t}, J=7.6 \mathrm{~Hz}, 1 \mathrm{H}), 4.45$ (q, $J=7.1 \mathrm{~Hz}$, $1 \mathrm{H}), 3.72(\mathrm{~s}, 3 \mathrm{H}), 2.89-2.70(\mathrm{~m}, 2 \mathrm{H}), 1.80-1.66(\mathrm{~m}, 2 \mathrm{H}), 1.41(\mathrm{t}, J=7.1 \mathrm{~Hz}, 3 \mathrm{H}), 1.06(\mathrm{t}, J=7.4 \mathrm{~Hz}$, $3 \mathrm{H}) ;{ }^{13} \mathrm{C}$ NMR $\left(101 \mathrm{MHz}, \mathrm{CDCl}_{3}\right) \delta: 166.7,159.2,147.4,139.8,131.2,126.3,125.9,122.3,119.4,114.7$, 61.6, 32.3, 29.5, 23.5, 14.5, 14.3. HRMS (ESI) m/z: $[\mathrm{M}+\mathrm{H}]^{+}$calcd for $\mathrm{C}_{16} \mathrm{H}_{20} \mathrm{NO}_{3}$ 274.1438; Found 274.1441 .

\section{Ethyl 4-isobutyl-1-methyl-2-oxo-1,2-dihydroquinoline-3-carboxylate (2d)}

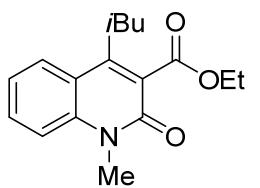

Prepared according to the general procedure from S1d $(819 \mathrm{mg}, 3.0 \mathrm{mmol}), \mathrm{MeI}$ (511 mg, $3.6 \mathrm{mmol}$ ) to provide the title compound as white solid (eluent: petroleum ether/ethyl acetate $=5: 1$ to $2: 1$ ), m.p. $60-61{ }^{\circ} \mathrm{C}, 0.80$ g, $93 \%$ yield, $R_{\mathrm{f}}=0.5$ (petroleum ether/ethyl acetate $=2: 1) .{ }^{1} \mathrm{H}$ NMR $\left(400 \mathrm{MHz}, \mathrm{CDCl}_{3}\right) \delta: 7.75(\mathrm{~d}, J=8.4$

$\mathrm{Hz}, 1 \mathrm{H}), 7.56$ (ddd, $J=8.4,7.2,1.2 \mathrm{~Hz}, 1 \mathrm{H}), 7.35(\mathrm{~d}, J=8.4 \mathrm{~Hz}, 1 \mathrm{H}), 7.25(\mathrm{t}, J=7.6 \mathrm{~Hz}, 1 \mathrm{H}), 4.45(\mathrm{q}$, $J=7.2 \mathrm{~Hz}, 2 \mathrm{H}), 3.68$ (s, 3H), 2.74 (d, $J=7.6 \mathrm{~Hz}, 2 \mathrm{H}), 2.17-1.99$ (m, 1H), $1.42(\mathrm{t}, J=7.2 \mathrm{~Hz}, 3 \mathrm{H}), 0.98$ (d, $J=6.8 \mathrm{~Hz}, 6 \mathrm{H}) ;{ }^{13} \mathrm{C}$ NMR $\left(101 \mathrm{MHz}, \mathrm{CDCl}_{3}\right) \delta: 166.6,159.0,146.4,139.4,131.1,126.9,126.1$, 122.1, 119.5, 114.6, 61.4, 38.5, 29.3, 29.2, 22.7, 14.1. HRMS (ESI) m/z: $[\mathrm{M}+\mathrm{H}]^{+}$calcd for $\mathrm{C}_{17} \mathrm{H}_{22} \mathrm{NO}_{3}$ 288.1594; Found 288.1596.

\section{Ethyl 4-benzyl-1-methyl-2-oxo-1,2-dihydroquinoline-3-carboxylate (2e)}

Prepared according to the general procedure from S1e (921 mg, $3.0 \mathrm{mmol})$, MeI (511 mg, $3.6 \mathrm{mmol})$ to 


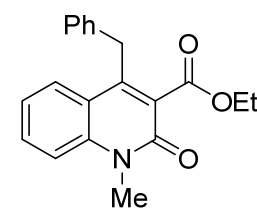

provide the title compound as white solid (eluent: petroleum ether/ethyl acetate $=$ 5:1 to $2: 1$ ), m.p. $169-170{ }^{\circ} \mathrm{C}, 0.88 \mathrm{~g}, 91 \%$ yield, $R_{\mathrm{f}}=0.3$ (petroleum ether/ethyl acetate $=2: 1) .{ }^{1} \mathrm{H}$ NMR $\left(400 \mathrm{MHz} \mathrm{CDCl}_{3}\right) \delta: 7.70(\mathrm{~d}, J=8.1 \mathrm{~Hz}, 1 \mathrm{H}), 7.54(\mathrm{t}, J=$ $7.9 \mathrm{~Hz}, 1 \mathrm{H}), 7.37$ (d, $J=8.4 \mathrm{~Hz}, 1 \mathrm{H}), 7.32-7.22(\mathrm{~m}, 4 \mathrm{H}), 7.23-7.10$ (m, 2H), 4.40 $(\mathrm{q}, J=7.1 \mathrm{~Hz}, 2 \mathrm{H}), 4.22(\mathrm{~s}, 2 \mathrm{H}), 3.75(\mathrm{~s}, 3 \mathrm{H}), 1.30(\mathrm{t}, J=7.1 \mathrm{~Hz}, 3 \mathrm{H}) ;{ }^{13} \mathrm{C} \mathrm{NMR}\left(101 \mathrm{MHz}, \mathrm{CDCl}_{3}\right) \delta$ : $166.6,159.2$, 144.6, 139.9, 137.5, 131.2, 128.7, 128.3, 128.0, 126.9, 126.7, 122.5, 119.6, 114.6, 61.8, 36.2, 29.6, 14.1. HRMS (ESI) m/z: $[\mathrm{M}+\mathrm{Na}]^{+}$calcd for $\mathrm{C}_{20} \mathrm{H}_{19} \mathrm{NO}_{3} \mathrm{Na} 344.1257$; Found 344.1263.

\section{Ethyl 1-methyl-2-oxo-4-phenethyl-1,2-dihydroquinoline-3-carboxylate (2f)}<smiles>CCOC(=O)c1c(CCc2ccccc2)c2ccccc2n(C)c1=O</smiles>

Prepared according to the general procedure from S1f (963 mg, $3.0 \mathrm{mmol})$, MeI (511 $\mathrm{mg}, 3.6 \mathrm{mmol}$ ) to provide the title compound as white solid (eluent: petroleum ether/ethyl acetate $=5: 1$ to 2:1), m.p. $96-97{ }^{\circ} \mathrm{C}, 0.98 \mathrm{~g}, 97 \%$ yield, $R_{\mathrm{f}}=0.5$ (petroleum ether/ethyl acetate $=2: 1) .{ }^{1} \mathrm{H}$ NMR $\left(400 \mathrm{MHz}, \mathrm{CDCl}_{3}\right) \delta: 7.84(\mathrm{~d}, J=8.1$ $\mathrm{Hz}, 1 \mathrm{H}), 7.61(\mathrm{t}, J=7.8 \mathrm{~Hz}, 1 \mathrm{H}), 7.40$ (d, $J=8.5 \mathrm{~Hz}, 1 \mathrm{H}), 7.36-7.19(\mathrm{~m}, 6 \mathrm{H}), 4.46(\mathrm{q}, J=7.1 \mathrm{~Hz}, 2 \mathrm{H})$, $3.72(\mathrm{~s}, 3 \mathrm{H}), 3.10(\mathrm{dd}, J=11.6,5.2 \mathrm{~Hz}, 2 \mathrm{H}), 2.98(\mathrm{dd}, J=11.2,5.3 \mathrm{~Hz}, 2 \mathrm{H}), 1.41(\mathrm{t}, J=7.1 \mathrm{~Hz}, 3 \mathrm{H}) ;{ }^{13} \mathrm{C}$ $\operatorname{NMR}\left(101 \mathrm{MHz}, \mathrm{CDCl}_{3}\right) \delta: 166.6,159.2,146.5,140.8,139.9,131.5,128.7,128.2,126.5,125.7,122.6$, $119.2,114.9,100.0,61.8,36.2,32.5,29.6,14.3$. HRMS (ESI) $\mathrm{m} / \mathrm{z}:[\mathrm{M}+\mathrm{H}]^{+}$calcd for $\mathrm{C}_{21} \mathrm{H}_{22} \mathrm{NO}_{3}$ 336.1594; Found 336.1599.

\section{Ethyl 1-methyl-2-oxo-4-pentyl-1,2-dihydroquinoline-3-carboxylate (2g)}<smiles>CCOC(=O)c1c(C=O)c2ccccc2n(C)c1=O</smiles>

Prepared according to the general procedure from S1g (861 mg, $3.0 \mathrm{mmol})$, MeI (511 $\mathrm{mg}, 3.6 \mathrm{mmol}$ ) to provide the title compound as white solid (eluent: petroleum ether/ethyl acetate $=5: 1$ to $2: 1$ ), m.p. $46-47{ }^{\circ} \mathrm{C}, 0.67 \mathrm{~g}, 74 \%$ yield, $R_{\mathrm{f}}=0.3$ (petroleum ether/ethyl acetate $=2: 1) .{ }^{1} \mathrm{H}$ NMR $\left(400 \mathrm{MHz}, \mathrm{CDCl}_{3}\right) \delta: 7.78(\mathrm{~d}, J=8.1$ $\mathrm{Hz}, 1 \mathrm{H}), 7.60$ (t, $J=7.8 \mathrm{~Hz}, 1 \mathrm{H}), 7.40$ (s, 1H), 7.29 (t, $J=7.6 \mathrm{~Hz}, 1 \mathrm{H}), 4.45$ (q, $J=8.2,7.7 \mathrm{~Hz}, 2 \mathrm{H})$, $3.71(\mathrm{~s}, 3 \mathrm{H}), 2.87-2.75(\mathrm{~m}, 2 \mathrm{H}), 1.76-1.62(\mathrm{~m}, 2 \mathrm{H}), 1.50-1.30(\mathrm{~m}, 7 \mathrm{H}), 0.92(\mathrm{t}, J=6.7 \mathrm{~Hz}, 3 \mathrm{H}) ;{ }^{13} \mathrm{C}$ NMR (101 MHz, $\left.\mathrm{CDCl}_{3}\right) \delta: 166.6,159.1,147.6,139.7,131.2,126.1,125.8,122.3,119.3,114.7,61.5$, 32.1, 30.3, 29.8, 29.4, 22.3, 14.2, 13.9. HRMS (ESI) m/z: $[\mathrm{M}+\mathrm{Na}]^{+}$calcd for $\mathrm{C}_{18} \mathrm{H}_{23} \mathrm{NO}_{3} \mathrm{Na} 324.1570$; Found 324.1576 .

\section{Ethyl 4-cyclopropyl-1-methyl-2-oxo-1,2-dihydroquinoline-3-carboxylate (2h)}<smiles>CCOC(=O)c1c(C2CC2)c2ccccc2n(C)c1=O</smiles>

Prepared according to the general procedure from $\mathbf{S 1 h}(771 \mathrm{mg}, 3.0 \mathrm{mmol})$, MeI (511 $\mathrm{mg}, 3.6 \mathrm{mmol}$ ) to provide the title compound as white solid (eluent: petroleum ether/ethyl acetate $=5: 1$ to $2: 1$ ), m.p. $80-81{ }^{\circ} \mathrm{C}, 0.61 \mathrm{~g}, 75 \%$ yield, $R_{\mathrm{f}}=0.3$ (petroleum ether/ethyl acetate $=2: 1) .{ }^{1} \mathrm{H}$ NMR $\left(400 \mathrm{MHz}, \mathrm{CDCl}_{3}\right) \delta: 8.25(\mathrm{dd}, J=$ 8.1, 1.2 Hz, 1H), 7.59 (t, $J=8.6 \mathrm{~Hz}, 1 \mathrm{H}), 7.35$ (d, $J=8.3 \mathrm{~Hz}, 1 \mathrm{H}), 7.28$ (t, $J=7.6 \mathrm{~Hz}, 1 \mathrm{H}), 4.46$ (q, $J=$ $7.2 \mathrm{~Hz}, 2 \mathrm{H}), 3.69$ (s, 3H), 2.13-2.02 (m, 1H), $1.43(\mathrm{t}, J=7.2 \mathrm{~Hz}, 3 \mathrm{H}), 1.15-1.07$ (m, 2H), 0.81-0.72 (m, $2 \mathrm{H}) ;{ }^{13} \mathrm{C} \mathrm{NMR}\left(101 \mathrm{MHz}, \mathrm{CDCl}_{3}\right) \delta: 166.4,159.4,146.9,139.2,131.2,127.5,126.8,122.2,121.0,114.3$, 61.5, 29.4, 14.2, 12.1, 6.6. HRMS (ESI) m/z: $[\mathrm{M}+\mathrm{H}]^{+}$calcd for $\mathrm{C}_{16} \mathrm{H}_{18} \mathrm{NO}_{3}$ 272.1281; Found 272.1282.

\section{Ethyl 1-methyl-2-oxo-4-phenyl-1,2-dihydroquinoline-3-carboxylate (2i) ${ }^{6}$}

Prepared according to the general procedure from S1i $(879 \mathrm{mg}, 3.0 \mathrm{mmol}), \mathrm{MeI}(511 \mathrm{mg}, 3.6 \mathrm{mmol})$ to 
<smiles>CCOC(=O)c1c(-c2ccccc2)c2ccccc2n(C)c1=O</smiles>

provide the title compound as white solid (eluent: petroleum ether/ethyl acetate $=$ 5:1 to $2: 1$ ), m.p. $120-121{ }^{\circ} \mathrm{C}, 0.77 \mathrm{~g}, 84 \%$ yield, $R_{\mathrm{f}}=0.2$ (petroleum ether/ethyl acetate $=2: 1) .{ }^{1} \mathrm{H}$ NMR $\left(400 \mathrm{MHz}, \mathrm{CDCl}_{3}\right) \delta: 7.62(\mathrm{ddd}, J=8.8,7.2,1.6 \mathrm{~Hz}, 1 \mathrm{H})$, $7.53-7.42(\mathrm{~m}, 4 \mathrm{H}), 7.41-7.28(\mathrm{~m}, 3 \mathrm{H}), 7.16$ (ddt, $J=7.2,6.4,0.8 \mathrm{~Hz}, 1 \mathrm{H}), 4.09$ (q, $J=7.2 \mathrm{~Hz}, 2 \mathrm{H}), 3.80(\mathrm{~s}, 3 \mathrm{H}), 0.97(\mathrm{t}, J=7.2 \mathrm{~Hz}, 3 \mathrm{H})$ (the spectra are in accordance with those of the compound reported in the literature).

\section{Ethyl 6-bromo-1,4-dimethyl-2-oxo-1,2-dihydroquinoline-3-carboxylate (2j)}<smiles>CCOC(=O)c1c(C)c2cc(Br)ccc2n(C)c1=O</smiles>
Prepared according to the general procedure from $\mathbf{S 1 j}$ ( $927 \mathrm{mg}, 3.0 \mathrm{mmol}$ ), MeI (511 mg, $3.6 \mathrm{mmol}$ ) to provide the title compound as white solid (eluent: petroleum ether/ethyl acetate $=5: 1)$, m.p. $115-116^{\circ} \mathrm{C}, 0.78 \mathrm{~g}, 81 \%$ yield, $R_{\mathrm{f}}=$ 0.6 (petroleum ether/ethyl acetate $=2: 1) .{ }^{1} \mathrm{H} \mathrm{NMR}\left(400 \mathrm{MHz}, \mathrm{CDCl}_{3}\right) \delta: 7.80$ $(\mathrm{d}, J=2.0 \mathrm{~Hz}, 1 \mathrm{H}), 7.65(\mathrm{dd}, J=9.0,2.0 \mathrm{~Hz}, 1 \mathrm{H}), 7.23(\mathrm{~d}, J=8.8 \mathrm{~Hz}, 1 \mathrm{H}), 4.45$ (q, $J=7.2 \mathrm{~Hz}, 2 \mathrm{H})$, $3.67(\mathrm{~s}, 3 \mathrm{H}), 2.40(\mathrm{~s}, 3 \mathrm{H}), 1.42$ (t, $J=7.2 \mathrm{~Hz}, 3 \mathrm{H}) ;{ }^{13} \mathrm{C} \mathrm{NMR}\left(101 \mathrm{MHz}, \mathrm{CDCl}_{3}\right) \delta: 166.2,158.6,142.1$, 138.2, 134.0, 128.2, 127.6, 121.9, 116.2, 115.4, 61.9, 29.6, 16.2, 14.2. HRMS (ESI) m/z: $[\mathrm{M}+\mathrm{H}]^{+}$calcd for $\mathrm{C}_{14} \mathrm{H}_{15} \mathrm{BrNO}_{3} 324.0230$; Found 324.0227.

\section{Ethyl 6-methoxy-1,4-dimethyl-2-oxo-1,2-dihydroquinoline-3-carboxylate (2k)}<smiles>CCOC(=O)c1c(C)c2cc(OC)ccc2n(C)c1=O</smiles>

Prepared according to the general procedure from S1k (783 mg, $3.0 \mathrm{mmol})$, MeI (511 mg, $3.6 \mathrm{mmol})$ to provide the title compound as light yellow solid (eluent: petroleum ether/ethyl acetate $=5: 1$ to 2:1), m.p. $119-120{ }^{\circ} \mathrm{C}, 0.63 \mathrm{~g}$, $76 \%$ yield, $R_{\mathrm{f}}=0.3$ (petroleum ether/ethyl acetate $\left.=2: 1\right) .{ }^{1} \mathrm{H}$ NMR $(400 \mathrm{MHz}$, $\left.\mathrm{CDCl}_{3}\right) \delta: 7.33(\mathrm{~d}, J=9.2 \mathrm{~Hz}, 1 \mathrm{H}), 7.26-7.16(\mathrm{~m}, 2 \mathrm{H}), 4.46(\mathrm{q}, J=7.2 \mathrm{~Hz}, 2 \mathrm{H}), 3.89(\mathrm{~s}, 3 \mathrm{H}), 3.71(\mathrm{~s}$, 3H), $2.45(\mathrm{~s}, 3 \mathrm{H}), 1.42\left(\mathrm{t}, J=7.2 \mathrm{~Hz}, 3 \mathrm{H} ;{ }^{13} \mathrm{C} \mathrm{NMR}\left(101 \mathrm{MHz}, \mathrm{CDCl}_{3}\right) \delta: 166.8,158.6,154.8,142.5\right.$, 133.9, 127.2, 121.2, 119.2, 115.7, 108.4, 61.7, 55.7, 29.5, 16.3, 14.2. HRMS (ESI) m/z: $[\mathrm{M}+\mathrm{H}]^{+}$calcd for $\mathrm{C}_{15} \mathrm{H}_{18} \mathrm{NO}_{4} 276.1230$; Found 276.1236.

\section{Ethyl 1-ethyl-4-methyl-2-oxo-1,2-dihydroquinoline-3-carboxylate (2l) ${ }^{7}$}<smiles>CCOC(=O)c1c(C)c2ccccc2n(CC)c1=O</smiles>

Prepared according to the general procedure from S1a (693 mg, $3.0 \mathrm{mmol})$, EtI (562 $\mathrm{mg}, 3.6 \mathrm{mmol}$ ) to provide the title compound as colorless oil (eluent: petroleum ether/ethyl acetate $=5: 1), 0.47 \mathrm{~g}, 60 \%$ yield, $R_{\mathrm{f}}=0.4$ (petroleum ether/ethyl acetate =2:1). ${ }^{1} \mathrm{H}$ NMR $\left(400 \mathrm{MHz}, \mathrm{CDCl}_{3}\right) \delta: 7.77(\mathrm{~d}, J=8.1 \mathrm{~Hz}, 1 \mathrm{H}), 7.60$ (t, $J=7.9 \mathrm{~Hz}$, 1H), 7.39 (d, $J=8.6 \mathrm{~Hz}, 1 \mathrm{H}), 7.33-7.21(\mathrm{~m}, 1 \mathrm{H}), 4.46$ (q, J=7.2 Hz, 2H), 4.36 (q, J=7.2 Hz, 2H), 2.46 $(\mathrm{s}, 3 \mathrm{H}), 1.42(\mathrm{t}, J=7.6 \mathrm{~Hz}, 3 \mathrm{H}), 1.35(\mathrm{t}, J=7.2 \mathrm{~Hz}, 3 \mathrm{H})$ (the spectra are in accordance with those of the compound reported in the literature).

\section{Ethyl 1-benzyl-4-methyl-2-oxo-1,2-dihydroquinoline-3-carboxylate (2m)}

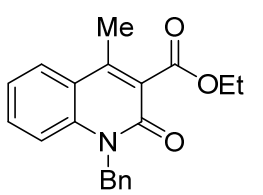

Prepared according to the general procedure from S1a (693 mg, $3.0 \mathrm{mmol}), \mathrm{BnBr}$ $(616 \mathrm{mg}, 3.6 \mathrm{mmol})$ to provide the title compound as white solid (eluent: petroleum ether/ethyl acetate $=5: 1$ ), m.p. $101-102{ }^{\circ} \mathrm{C}, 0.78 \mathrm{~g}, 72 \%$ yield, $R_{\mathrm{f}}=0.4$ (petroleum ether/ethyl acetate $=2: 1) .{ }^{1} \mathrm{H} \mathrm{NMR}\left(400 \mathrm{MHz}, \mathrm{CDCl}_{3}\right) \delta: 7.77(\mathrm{dd}, J=8.2,1.4 \mathrm{~Hz}$,

1H), 7.46 (ddd, $J=8.8,7.2,1.6 \mathrm{~Hz}, 1 \mathrm{H}), 7.33-7.17$ (m, 7H), 5.55 (s, 2H), 4.47 (q, $J=7.2 \mathrm{~Hz}, 2 \mathrm{H}), 2.51$ $(\mathrm{s}, 3 \mathrm{H}), 1.43$ (t, $J=7.2 \mathrm{~Hz}, 3 \mathrm{H}) ;{ }^{13} \mathrm{C} \mathrm{NMR}\left(101 \mathrm{MHz}, \mathrm{CDCl}_{3}\right) \delta: 166.8,159.3,144.0,138.8,136.1,131.4$, $128.8,127.4,126.7,126.7,125.9,122.5,120.6,115.4,61.8,46.0,16.4,14.3$. HRMS (ESI) m/z: $[\mathrm{M}+\mathrm{H}]^{+}$ 
calcd for $\mathrm{C}_{20} \mathrm{H}_{20} \mathrm{NO}_{3}$ 322.1438; Found 322.1443.

4-Substituted 3-ethoxycarbonyl quinolin-2-ones $\mathbf{2 n - 0}$ were synthesized from the known starting material $N$-phenyl $o$-aminoaryl ketones according to the known literature. ${ }^{8}$

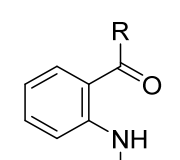

$\mathrm{R}=\mathrm{Me}, \mathrm{Et}$

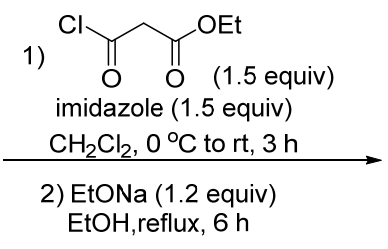

EtOH,reflux, $\mathrm{h}$

EtOH, reflux, 6 h

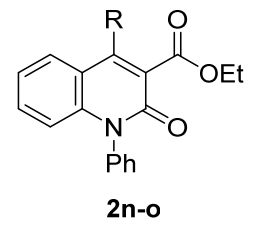

General procedure: To a solution of $N$-phenyl $o$-aminoaryl ketone $(3.0 \mathrm{mmol})$ and imidazole (306 $\mathrm{mg}$, $4.5 \mathrm{mmol})$ in $\mathrm{CH}_{2} \mathrm{Cl}_{2}(20 \mathrm{~mL})$ was added ethyl malonyl chloride $(560 \mu \mathrm{L}, 4.5 \mathrm{mmol})$ dropwise with stirring at $0{ }^{\circ} \mathrm{C}$. The resulting reaction mixture was stirred at room temperature for $3 \mathrm{~h}$. After filtering off the light yellow precipitate, the filtrate was concentrated in vacuo. The residue was then redissolved with a solution of EtONa $(245 \mathrm{mg}, 3.6 \mathrm{mmol})$ in absolute $\mathrm{EtOH}(15 \mathrm{~mL})$ and the the resulting reaction mixture was heated to reflux in an oil bath for $6 \mathrm{~h}$. The reaction mixture was then diluted with water $(10 \mathrm{~mL})$, acidified with $2 \mathrm{~N} \mathrm{HCl}(10 \mathrm{~mL})$, and extracted with $\mathrm{CH}_{2} \mathrm{Cl}_{2}(3 \times 20 \mathrm{~mL})$. The combined extracts were washed with brine $(20 \mathrm{~mL})$, dried over $\mathrm{MgSO}_{4}$, concentrated in vacuo. The residue was purified by chromatography on silica gel with petroleum ether/ethyl acetate (5:1 to 2:1) as eluent to afford corresponding product $\mathbf{2 n - 0}$ as white solid.

\section{Ethyl 4-methyl-2-oxo-1-phenyl-1,2-dihydroquinoline-3-carboxylate (2n)}<smiles>CCOC(=O)c1c(N)c2ccccc2[nH]c1=O</smiles>

Prepared according to the general procedure from 1-(2-(phenylamino)phenyl)ethan1-one (633 mg, $3.0 \mathrm{mmol}$ ), to provide the title compound as white solid (eluent: petroleum ether/ethyl acetate $=5: 1$ to 2:1), m.p. $113-114{ }^{\circ} \mathrm{C}, 0.69 \mathrm{~g}, 75 \%$ yield, $R_{\mathrm{f}}$ $=0.4$ (petroleum ether/ethyl acetate $=2: 1) .{ }^{1} \mathrm{H}$ NMR $\left(400 \mathrm{MHz}, \mathrm{CDCl}_{3}\right) \delta: 7.79(\mathrm{~d}$, $J=8.0 \mathrm{~Hz}, 1 \mathrm{H}), 7.57(\mathrm{t}, J=7.6 \mathrm{~Hz}, 2 \mathrm{H}), 7.49(\mathrm{t}, J=7.6 \mathrm{~Hz}, 1 \mathrm{H}), 7.36(\mathrm{t}, J=7.8 \mathrm{~Hz}, 1 \mathrm{H}), 7.31-7.20(\mathrm{~m}$, 3H), $6.69(\mathrm{~d}, J=8.4 \mathrm{~Hz}, 1 \mathrm{H}), 4.43$ (q, $J=7.2 \mathrm{~Hz}, 2 \mathrm{H}), 2.55$ (s, $3 \mathrm{H}), 1.40(\mathrm{t}, J=7.2 \mathrm{~Hz}, 3 \mathrm{H}) ;{ }^{13} \mathrm{C}$ NMR (101 MHz, $\left.\mathrm{CDCl}_{3}\right) \delta: 166.5,159.0,144.4,140.5,137.1,131.0,130.1,129.0,128.9,127.0,125.5,122.6$, 120.1, 116.4, 61.8, 16.5, 14.2. HRMS (ESI) m/z: $[\mathrm{M}+\mathrm{H}]^{+}$calcd for $\mathrm{C}_{19} \mathrm{H}_{18} \mathrm{NO}_{3} 308.1281$; Found 308.1284 .

\section{Ethyl 4-ethyl-2-oxo-1-phenyl-1,2-dihydroquinoline-3-carboxylate (2o)}<smiles>CCOC(=O)c1c(CC)c2ccccc2[nH]c1=O</smiles>

Prepared according to the general procedure from 1-(2(phenylamino)phenyl)propan-1-one $(675 \mathrm{mg}, 3.0 \mathrm{mmol})$, to provide the title compound as white solid (eluent: petroleum ether/ethyl acetate $=5: 1$ ), m.p. 117-118 ${ }^{\circ} \mathrm{C}, 0.75 \mathrm{~g}, 78 \%$ yield, $R_{\mathrm{f}}=0.6$ (petroleum ether/ethyl acetate $\left.=2: 1\right) .{ }^{1} \mathrm{H}$ NMR $(400$ $\left.\mathrm{MHz}, \mathrm{CDCl}_{3}\right) \delta: 7.82(\mathrm{dd}, J=8.2,1.4 \mathrm{~Hz}, 1 \mathrm{H}), 7.62-7.54(\mathrm{~m}, 2 \mathrm{H}), 7.54-7.46(\mathrm{~m}, 1 \mathrm{H}), 7.36(\mathrm{ddd}, J=$ 8.6, 7.2, 1.6 Hz, 1H), 7.31-7.21 (m, 3H), 6.72 (dd, $J=8.4,1.2 \mathrm{~Hz}, 1 \mathrm{H}$ ), 4.43 (q, $J=7.2 \mathrm{~Hz}, 2 \mathrm{H}$ ), 2.92 (q, $J=7.6 \mathrm{~Hz}, 2 \mathrm{H}), 1.46-1.31(\mathrm{~m}, 6 \mathrm{H}) ;{ }^{13} \mathrm{C} \mathrm{NMR}\left(101 \mathrm{MHz}, \mathrm{CDCl}_{3}\right) \delta: 166.4,159.2,149.8,141.0,137.2$, 130.8, 130.1, 129.0, 128.9, 126.4, 125.4, 122.6, 118.9, 116.7, 61.7, 23.8, 14.4, 14.2. HRMS (ESI) m/z: $[\mathrm{M}+\mathrm{H}]^{+}$calcd for $\mathrm{C}_{20} \mathrm{H}_{20} \mathrm{NO}_{3} 322.1438$; Found 322.1439.

\section{Preparation of 4-substituted 3-ethoxycarbonyl coumarins 4}


4-Substituted 3-ethoxycarbonyl coumarins 4a-o were prepared by Knoevenagel condensation of $o$ hydroxyaryl ketone with diethyl malonate according to literature method. ${ }^{6}$

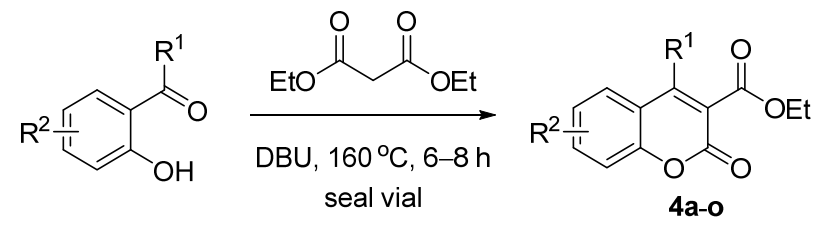

General procedure: A mixture of o-hydroxyaryl ketone $(5.0 \mathrm{mmol})$, diethyl malonate $(2.40 \mathrm{~g}, 15.0$ $\mathrm{mmol})$ and DBU $(75 \mu \mathrm{L}, 0.5 \mathrm{mmol})$ in a sealed vial was stirred at $160^{\circ} \mathrm{C}$ in an oil bath for $6-8 \mathrm{~h}$. After removal of diethyl malonate under reduced pressure, the residue was purified by flash chromatography on a silica gel with petroleum ether /ethyl acetate (10:1 to 4:1) as an eluent to afford the desired product 4a-o as a colorless oil or a white solid.

\section{Ethyl 4-methyl-2-oxo-2H-chromene-3-carboxylate (4a) ${ }^{9}$}<smiles>CCOC(=O)c1c(OC)c2ccccc2oc1=O</smiles>

Prepared according to the general procedure from 1-(2-hydroxyphenyl)ethan-1-one (680 mg, $5.0 \mathrm{mmol}$ ), to provide the title compound as white solid (eluent: petroleum ether/ethyl acetate $=10: 1$ to $4: 1)$, m.p. $86-87^{\circ} \mathrm{C}, 6 \mathrm{~h}, 0.63 \mathrm{~g}, 54 \%$ yield, $R_{\mathrm{f}}=0.4$ (petroleum ether/ethyl acetate $=5: 1) .{ }^{1} \mathrm{H}$ NMR $\left(400 \mathrm{MHz}, \mathrm{CDCl}_{3}\right) \delta: 7.2-7.65(\mathrm{~m}, 1 \mathrm{H}), 7.59(\mathrm{ddd}, J=$ 8.6, 7.2, 1.6 Hz, 1H), 7.39-7.31 (m, 2H), 4.44 (q, J=7.2 Hz, 2H), 2.49 (s, 3H), 1.41 (t, $J=7.2 \mathrm{~Hz}, 3 \mathrm{H})$ (the spectra are in accordance with those of the compound reported in the literature).

\section{Ethyl 4-ethyl-2-oxo-2H-chromene-3-carboxylate (4b) ${ }^{10}$}<smiles>CCOC(=O)c1c(OCC)c2ccccc2oc1=O</smiles>

Prepared according to the general procedure from 1-(2-hydroxyphenyl)propan-1-one (750 mg, $5.0 \mathrm{mmol}$ ), to provide the title compound as white solid (eluent: petroleum ether/ethyl acetate $=10: 1$ to $4: 1)$, m.p. $42-43{ }^{\circ} \mathrm{C}, 6 \mathrm{~h}, 0.38 \mathrm{~g}, 31 \%$ yield, $R_{\mathrm{f}}=0.5$ (petroleum ether/ethyl acetate = 3:1). ${ }^{1} \mathrm{H}$ NMR $\left(400 \mathrm{MHz}, \mathrm{CDCl}_{3}\right)$ ): 7.74-7.67 (m, 1H), 7.62-7.54 (m, $1 \mathrm{H}), 7.40-7.30(\mathrm{~m}, 2 \mathrm{H}), 4.48-4.39(\mathrm{~m}, 2 \mathrm{H}), 2.88-2.77(\mathrm{~m}, 2 \mathrm{H}), 1.44-1.30(\mathrm{~m}, 6 \mathrm{H})$ (the spectra are in accordance with those of the compound reported in the literature).

\section{Ethyl 2-oxo-4-propyl-2H-chromene-3-carboxylate (4c) ${ }^{6}$}<smiles>CCOC(=O)c1c(OCC)c2ccccc2oc1=O</smiles>

Prepared according to the general procedure from 1-(2-hydroxyphenyl)butan-1-one ( $820 \mathrm{mg}, 5.0 \mathrm{mmol}$ ), to provide the title compound as white solid (eluent: petroleum ether/ethyl acetate $=10: 1$ to $6: 1)$, m.p. $58-59^{\circ} \mathrm{C}, 6 \mathrm{~h}, 0.46 \mathrm{~g}, 35 \%$ yield, $R_{\mathrm{f}}=0.6$ (petroleum ether/ethyl acetate $=3: 1) .{ }^{1} \mathrm{H}$ NMR $\left(400 \mathrm{MHz}, \mathrm{CDCl}_{3}\right) \delta: 7.68(\mathrm{dt}, J=8.0,1.8 \mathrm{~Hz}, 1 \mathrm{H}), 7.57$ (ddt, $J=8.4,7.2,1.5 \mathrm{~Hz}, 1 \mathrm{H}), 7.40-7.29(\mathrm{~m}, 2 \mathrm{H}), 4.44$ (q, $J=7.2 \mathrm{~Hz}, 2 \mathrm{H}), 2.82-2.72(\mathrm{~m}, 2 \mathrm{H}), 1.82$ $1.67(\mathrm{~m}, 2 \mathrm{H}), 1.41(\mathrm{t}, J=7.2 \mathrm{~Hz}, 3 \mathrm{H}), 1.07(\mathrm{t}, J=7.4 \mathrm{~Hz}, 3 \mathrm{H})$ (the spectra are in accordance with those of the compound reported in the literature).

\section{Ethyl 4-isobutyl-2-oxo-2H-chromene-3-carboxylate (4d)}<smiles>CCOC(=O)c1c(Br)c2ccccc2oc1=O</smiles>

Prepared according to the general procedure from 1-(2-hydroxyphenyl)-3methylbutan-1-one ( $890 \mathrm{mg}, 5.0 \mathrm{mmol})$, to provide the title compound as colorless oil (eluent: petroleum ether/ethyl acetate $=10: 1$ to $4: 1), 8 \mathrm{~h}, 0.53 \mathrm{~g}, 39 \%$ yield, $R_{\mathrm{f}}=$ $0.4($ petroleum ether/ethyl acetate $=5: 1) .{ }^{1} \mathrm{H}$ NMR $\left(400 \mathrm{MHz}, \mathrm{CDCl}_{3}\right) \delta: 7.69(\mathrm{dd}, J=8.0,1.6 \mathrm{~Hz}, 1 \mathrm{H})$, 7.57 (ddd, $J=8.4,7.2,1.6 \mathrm{~Hz}, 1 \mathrm{H}), 7.41-7.30$ (m, 2H), 4.43 (q, $J=7.2 \mathrm{~Hz}, 2 \mathrm{H}), 2.77$ (d, $J=7.2 \mathrm{~Hz}$, 
2H), 2.17-2.00 (m, 1H), 1.40 (t, $J=7.2 \mathrm{~Hz}, 3 \mathrm{H}), 1.01(\mathrm{~d}, J=6.4 \mathrm{~Hz}, 6 \mathrm{H}) ;{ }^{13} \mathrm{C} \mathrm{NMR}\left(101 \mathrm{MHz}, \mathrm{CDCl}_{3}\right)$

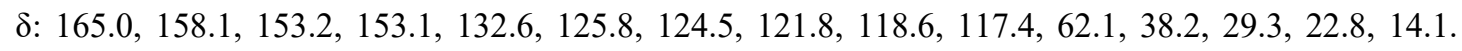
HRMS (ESI) m/z: [M+Na] $]^{+}$calcd for $\mathrm{C}_{16} \mathrm{H}_{18} \mathrm{O}_{4} \mathrm{Na} \quad$ 297.1097; Found 297.1102.

\section{Ethyl 4-isopropyl-2-oxo-2H-chromene-3-carboxylate (4e)}

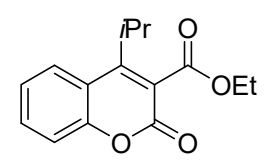

Prepared according to the general procedure from 1-(2-hydroxyphenyl)-2methylpropan-1-one $(820 \mathrm{mg}, 5.0 \mathrm{mmol})$, to provide the title compound as white solid (eluent: petroleum ether/ethyl acetate $=10: 1$ to $4: 1$ ), m.p. $74-75^{\circ} \mathrm{C} .7 \mathrm{~h}, 0.66$ g, $51 \%$ yield, $R_{\mathrm{f}}=0.6$ (petroleum ether/ethyl acetate $\left.=2: 1\right) .{ }^{1} \mathrm{H}$ NMR $\left(400 \mathrm{MHz}, \mathrm{CDCl}_{3}\right) \delta: 7.88(\mathrm{~d}, J=$ $7.6 \mathrm{~Hz}, 1 \mathrm{H}), 7.61-7.51(\mathrm{~m}, 1 \mathrm{H}), 7.40-7.27(\mathrm{~m}, 2 \mathrm{H}), 4.43(\mathrm{q}, J=7.2 \mathrm{~Hz}, 2 \mathrm{H}), 3.50-3.26(\mathrm{~m}, 1 \mathrm{H}), 1.47$ $(\mathrm{d}, J=7.2 \mathrm{~Hz}, 6 \mathrm{H}), 1.41(\mathrm{t}, J=7.2 \mathrm{~Hz}, 3 \mathrm{H}) ;{ }^{13} \mathrm{C} \mathrm{NMR}\left(101 \mathrm{MHz}, \mathrm{CDCl}_{3}\right) \delta: 165.5,158.6,157.0,153.2$, 132.1, 125.8, 124.4, 120.6, 117.7, 117.6, 62.0, 31.0, 20.8, 14.0. HRMS (ESI) $\mathrm{m} / \mathrm{z}:[\mathrm{M}+\mathrm{Na}]^{+}$calcd for $\mathrm{C}_{15} \mathrm{H}_{16} \mathrm{O}_{4} \mathrm{Na} 283.0941$; Found 283.0944.

\section{Ethyl 4-cyclohexyl-2-oxo-2H-chromene-3-carboxylate (4f)}

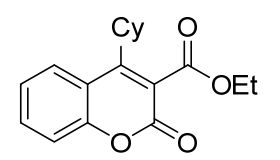

Prepared according to the general procedure from cyclohexyl(2hydroxyphenyl)methanone $(1.02 \mathrm{~g}, 5.0 \mathrm{mmol})$, to provide the title compound as white solid (eluent: petroleum ether/ethyl acetate $=10: 1$ to $6: 1$ ), m.p. $124-125^{\circ} \mathrm{C} .8$ h, $0.79 \mathrm{~g}, 53 \%$ yield, $R_{\mathrm{f}}=0.7$ (petroleum ether/ethyl acetate $\left.=3: 1\right) .{ }^{1} \mathrm{H}$ NMR $\left(400 \mathrm{MHz}, \mathrm{CDCl}_{3}\right) \delta: 7.89$ (s, 1H), 7.55 (t, $J=7.6 \mathrm{~Hz}, 1 \mathrm{H}), 7.43-7.15(\mathrm{~m}, 2 \mathrm{H}), 4.44(\mathrm{q}, J=7.2 \mathrm{~Hz}, 2 \mathrm{H}), 3.00(\mathrm{~s}, 1 \mathrm{H}), 2.09-1.61(\mathrm{~m}$, 7H), 1.53-1.17 (m, 6H); ${ }^{13} \mathrm{C}$ NMR (101 MHz, $\left.\mathrm{CDCl}_{3}\right) \delta: 165.5,158.6,155.5,153.0,132.1,125.0,124.4$, 120.6, 117.9, 117.3, 61.9, 53.6, 30.6, 26.7, 25.7, 14.0. HRMS (ESI) m/z: $[\mathrm{M}+\mathrm{Na}]^{+}$calcd for $\mathrm{C}_{18} \mathrm{H}_{20} \mathrm{O}_{4} \mathrm{Na}$ 323.1254; Found 323.1257.

\section{Ethyl 4-benzyl-2-oxo-2H-chromene-3-carboxylate (4g)}<smiles>CCOC(=O)c1c(Cc2ccccc2)c2ccccc2oc1=O</smiles>

Prepared according to the general procedure from 1-(2-hydroxyphenyl)-2phenylethan-1-one (1.06 g, $5.0 \mathrm{mmol})$, to provide the title compound as white solid (eluent: petroleum ether/ethyl acetate $=10: 1$ to 4:1), m.p. $101-102{ }^{\circ} \mathrm{C} .6 \mathrm{~h}, 0.55 \mathrm{~g}$, $36 \%$ yield, $R_{\mathrm{f}}=0.6$ (petroleum ether/ethyl acetate $\left.=2: 1\right)$. ${ }^{1} \mathrm{H} \mathrm{NMR}\left(400 \mathrm{MHz}, \mathrm{CDCl}_{3}\right)$ $\delta: 7.60(\mathrm{dd}, J=8.0,1.6 \mathrm{~Hz}, 1 \mathrm{H}), 7.52-7.46(\mathrm{~m}, 1 \mathrm{H}), 7.34-7.26(\mathrm{~m}, 5 \mathrm{H}), 7.25-7.16(\mathrm{~m}, 2 \mathrm{H}), 4.38$ (q, $J=$ $7.2 \mathrm{~Hz}, 2 \mathrm{H}), 4.20$ (s, 2H), 1.30 (t, J=7.2 Hz, 3H); ${ }^{13} \mathrm{C} \mathrm{NMR}\left(101 \mathrm{MHz}, \mathrm{CDCl}_{3}\right) \delta: 164.8,158.0,153.4$, 151.0, 136.3, 132.6, 129.0, 128.4, 127.1, 126.4, 124.7, 122.7, 118.3, 117.2, 62.3, 35.8, 14.0. HRMS (ESI) $\mathrm{m} / \mathrm{z}:[\mathrm{M}+\mathrm{Na}]^{+}$calcd for $\mathrm{C}_{19} \mathrm{H}_{16} \mathrm{O}_{4} \mathrm{Na} 331.0941$; Found 331.0944 .

\section{Ethyl 2-oxo-4-phenethyl-2H-chromene-3-carboxylate (4h)}

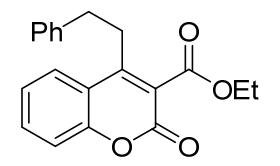

Prepared according to the general procedure from 1-(2-hydroxyphenyl)-3phenylpropan-1-one $(1.13 \mathrm{~g}, 5.0 \mathrm{mmol})$, to provide the title compound as white solid (eluent: petroleum ether/ethyl acetate $=10: 1$ to $4: 1$ ), m.p. $118-119^{\circ} \mathrm{C} .6 \mathrm{~h}, 1.03 \mathrm{~g}$, $64 \%$ yield, $R_{\mathrm{f}}=0.6$ (petroleum ether/ethyl acetate $\left.=2: 1\right) .{ }^{1} \mathrm{H}$ NMR $\left(400 \mathrm{MHz}, \mathrm{CDCl}_{3}\right) \delta: 7.73(\mathrm{~d}, J=8.0$ $\mathrm{Hz}, 1 \mathrm{H}), 7.60$ (t, $J=7.8 \mathrm{~Hz}, 1 \mathrm{H}), 7.47-7.30(\mathrm{~m}, 4 \mathrm{H}), 7.25$ (d, $J=7.8 \mathrm{~Hz}, 3 \mathrm{H}), 4.42$ (q, $J=7.2 \mathrm{~Hz}, 2 \mathrm{H}$ ), 3.16-3.07 (m, 2H), 3.04-2.94 (m, 2H), $1.39(\mathrm{t}, J=7.2 \mathrm{~Hz}, 3 \mathrm{H}) ;{ }^{13} \mathrm{C}$ NMR $\left(101 \mathrm{MHz}, \mathrm{CDCl}_{3}\right) \delta: 164.8$, 157.9, 153.5, 153.0, 140.1, 132.8, 128.8, 128.2, 126.7, 125.3, 124.8, 121.2, 118.1, 117.5, 62.2, 35.7, 32.1, 14.2. HRMS (ESI) m/z: [M+Na] $]^{+}$calcd for $\mathrm{C}_{20} \mathrm{H}_{18} \mathrm{O}_{4} \mathrm{Na}$ 345.1097; Found 345.1101. 


\section{Ethyl 4-(4-ethoxy-4-oxobutyl)-2-oxo-2H-chromene-3-carboxylate (4i)}

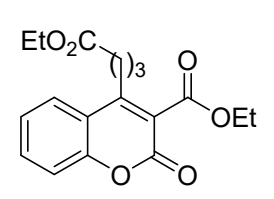

Prepared according to the general procedure from ethyl 5-(2-hydroxyphenyl)-5oxopentanoate $(1.18 \mathrm{~g}, 5.0 \mathrm{mmol})$, to provide the title compound as colorless oil (eluent: petroleum ether/ethyl acetate $=10: 1$ to $4: 1), 6 \mathrm{~h}, 0.55 \mathrm{~g}, 33 \%$ yield, $R_{\mathrm{f}}=0.4$ (petroleum ether/ethyl acetate $=2: 1) .{ }^{1} \mathrm{H}$ NMR $\left(400 \mathrm{MHz}, \mathrm{CDCl}_{3}\right) \delta: 7.88(\mathrm{~d}, J=8.0$ $\mathrm{Hz}, 1 \mathrm{H}), 7.63-7.54$ (m, 1H), 7.40-7.33 (m, 2H), 4.44 (q, J=7.2 Hz, 2H), 4.18 (q, J= 7.2 Hz, 2H), 2.92$2.82(\mathrm{~m}, 2 \mathrm{H}), 2.49(\mathrm{t}, J=6.8 \mathrm{~Hz}, 3 \mathrm{H}), 2.08-1.96(\mathrm{~m}, 2 \mathrm{H}), 1.41(\mathrm{t}, J=7.2 \mathrm{~Hz}, 3 \mathrm{H}), 1.30$ (t, $J=7.2 \mathrm{~Hz}$, $3 \mathrm{H}) ;{ }^{13} \mathrm{C}$ NMR (101 MHz, $\left.\mathrm{CDCl}_{3}\right) \delta: 172.8,164.7,157.9,153.3,153.2,132.7,125.7,124.9,121.2,118.1$, $117.3,62.1,60.6,33.5,29.4,24.5,14.2,14.1$. HRMS (ESI) $\mathrm{m} / \mathrm{z}$ : $[\mathrm{M}+\mathrm{Na}]^{+}$calcd for $\mathrm{C}_{18} \mathrm{H}_{20} \mathrm{O}_{6} \mathrm{Na}$ 355.1152; Found 355.1156.

\section{Ethyl 4-(2-((tert-butoxycarbonyl)(methyl)amino)ethyl)-2-oxo-2H-chromene-3-carboxylate (4j)}<smiles>CCOC(=O)c1c(CN(C)C(=O)OC)c2ccccc2oc1=O</smiles>

Prepared according to the general procedure from tert-butyl (3-(2-hydroxyphenyl)3-oxopropyl)(methyl)carbamate $(1.40 \mathrm{~g}, 5.0 \mathrm{mmol})$, to provide the title compound as white solid (eluent: petroleum ether/ethyl acetate $=10: 1$ to 4:1), m.p. $68-69^{\circ} \mathrm{C} .6$ h, $0.90 \mathrm{~g}, 48 \%$ yield, $R_{\mathrm{f}}=0.4$ (petroleum ether/ethyl acetate $\left.=2: 1\right) .{ }^{1} \mathrm{H}$ NMR $(400$ $\left.\mathrm{MHz}, \mathrm{CDCl}_{3}\right) \delta: 8.03(\mathrm{~d}, J=7.4 \mathrm{~Hz}, 0.5 \mathrm{H}), 7.80(\mathrm{~d}, J=7.4 \mathrm{~Hz}, 0.5 \mathrm{H}), 7.60(\mathrm{t}, J=7.4 \mathrm{~Hz}, 1 \mathrm{H}), 7.46-$ $7.28(\mathrm{~m}, 2 \mathrm{H}), 4.45$ (q, $J=7.2 \mathrm{~Hz}, 2 \mathrm{H}), 3.56(\mathrm{q}, J=7.2 \mathrm{~Hz}, 2 \mathrm{H}), 3.07$ (q, J=7.2 Hz, 2H), 2.92 (s, $1.5 \mathrm{H}$ ), $2.88(\mathrm{~s}, 1.5 \mathrm{H}), 1.56-1.36(\mathrm{~m}, 12 \mathrm{H})$ (mixture of rotamers); ${ }^{13} \mathrm{C} \mathrm{NMR}\left(101 \mathrm{MHz}, \mathrm{CDCl}_{3}\right)$ 8: 164.2, 156.9, 155.2, 154.4, 152.8, 150.7, 132.5, 125.6, 125.1, 124.7, 124.4, 121.4, 121.3, 117.9, 116.8, 116.6, 79.3, 79.1, 61.7, 48.4, 48.1, 34.8, 33.9, 27.9, 13.8 (mixture of rotamers). HRMS (ESI) m/z: [M+Na] ${ }^{+}$calcd for $\mathrm{C}_{20} \mathrm{H}_{25} \mathrm{NO}_{6} \mathrm{Na} 398.1574$; Found 398.1578.

\section{Ethyl 7-methoxy-2-oxo-4-pentyl-2H-chromene-3-carboxylate (4k)}<smiles>CCOC(=O)c1c(C=O)c2ccc(OC)cc2oc1=O</smiles>
Prepared according to the general procedure from 1-(2-hydroxy-4methoxyphenyl)hexan-1-one $(1.11 \mathrm{~g}, 5.0 \mathrm{mmol})$, to provide the title compound as white solid (eluent: petroleum ether/ethyl acetate $=10: 1$ to 6:1), m.p. 53-54 ${ }^{\circ} \mathrm{C} .6 \mathrm{~h}, 1.19 \mathrm{~g}, 75 \%$ yield, $R_{\mathrm{f}}=0.7$ (petroleum ether/ethyl acetate $\left.=2: 1\right) .{ }^{1} \mathrm{H}$ NMR $\left(400 \mathrm{MHz}, \mathrm{CDCl}_{3}\right) \delta: 7.56(\mathrm{~d}, J=8.8 \mathrm{~Hz}, 1 \mathrm{H}), 6.90(\mathrm{dd}, J=8.8,2.4 \mathrm{~Hz}, 1 \mathrm{H}), 6.83(\mathrm{~d}, J=2.4 \mathrm{~Hz}$, $1 \mathrm{H}), 4.41$ (q, $J=7.2 \mathrm{~Hz}, 2 \mathrm{H}), 3.89$ (s, 3H), 2.79-2.73 (m, 2H), 1.72-1.64 (m, 2H), 1.48-1.32 (m, 7H), $0.92(\mathrm{t}, J=7.2 \mathrm{~Hz}, 3 \mathrm{H}) ;{ }^{13} \mathrm{C} \mathrm{NMR}\left(101 \mathrm{MHz}, \mathrm{CDCl}_{3}\right) \delta: 165.2,163.3,158.5,155.3,154.9,126.5,117.5$, 113.0, 111.6, 100.9, 61.9, 55.8, 32.1, 30.1, 29.6, 22.3, 14.2, 13.9. HRMS (ESI) m/z: $[\mathrm{M}+\mathrm{Na}]^{+}$calcd for $\mathrm{C}_{18} \mathrm{H}_{22} \mathrm{O}_{5} \mathrm{Na}$ 341.1359; Found 341.1362.

\section{Ethyl 4-cyclopropyl-2-oxo-2H-chromene-3-carboxylate (4I)}<smiles>CCOC(=O)c1c(C2CC2)c2ccccc2oc1=O</smiles>

Prepared according to the general procedure from cyclopropyl(2hydroxyphenyl)methanone $(810 \mathrm{mg}, 5.0 \mathrm{mmol})$, to provide the title compound as white solid (eluent: petroleum ether/ethyl acetate $=10: 1$ to $4: 1$ ), m.p. $73-74^{\circ} \mathrm{C} .8 \mathrm{~h}$, $0.79 \mathrm{~g}, 61 \%$ yield, $R_{\mathrm{f}}=0.3$ (petroleum ether/ethyl acetate $\left.=5: 1\right) .{ }^{1} \mathrm{H} \mathrm{NMR}(400 \mathrm{MHz}$, $\left.\mathrm{CDCl}_{3}\right) \delta: 8.09$ (d, $\left.J=7.7 \mathrm{~Hz}, 1 \mathrm{H}\right), 7.57$ (t, $\left.J=7.4 \mathrm{~Hz}, 1 \mathrm{H}\right), 7.41-7.22(\mathrm{~m}, 2 \mathrm{H}), 4.44$ (q, $\left.J=7.2 \mathrm{~Hz}, 2 \mathrm{H}\right)$, 2.14-1.99 (m, 1H), 1.42 (t, $J=7.2 \mathrm{~Hz}, 3 \mathrm{H}), 1.16$ (q, $J=5.6 \mathrm{~Hz}, 2 \mathrm{H}), 0.78$ (q, $J=5.6 \mathrm{~Hz}, 2 \mathrm{H}) ;{ }^{13} \mathrm{C}$ NMR $\left(101 \mathrm{MHz}, \mathrm{CDCl}_{3}\right) \delta: 164.7,158.3,153.1,152.8,132.5,126.4,124.5,122.4,119.8,116.9,62.0,14.1$, 12.1, 6.7. HRMS (ESI) m/z: [M+Na] $]^{+}$calcd for $\mathrm{C}_{15} \mathrm{H}_{14} \mathrm{O}_{4} \mathrm{Na} 281.0784$; Found 281.0788. 
Ethyl 4-cyclopropyl-7-methoxy-2-oxo-2H-chromene-3-carboxylate (4m)<smiles>CCOC(=O)c1c(C2CC2)c2ccc(OC)cc2oc1=O</smiles>

Prepared according to the general procedure from cyclopropyl(2-hydroxy-4methoxyphenyl)methanone ( $960 \mathrm{mg}, 5.0 \mathrm{mmol}$ ), to provide the title compound as colorless oil (eluent: petroleum ether/ethyl acetate $=10: 1$ to $4: 1$ ), $8 \mathrm{~h}, 0.62$ g, $43 \%$ yield, $R_{\mathrm{f}}=0.6$ (petroleum ether/ethyl acetate $\left.=2: 1\right) .{ }^{1} \mathrm{H} \mathrm{NMR}(400 \mathrm{MHz}$, $\left.\mathrm{CDCl}_{3}\right) \delta: 7.98(\mathrm{~d}, J=8.8 \mathrm{~Hz}, 1 \mathrm{H}), 6.90(\mathrm{dd}, J=8.8,2.4 \mathrm{~Hz}, 1 \mathrm{H}), 6.76(\mathrm{~d}, J=2.4 \mathrm{~Hz}, 1 \mathrm{H}), 4.42(\mathrm{q}, J=$ $7.2 \mathrm{~Hz}, 2 \mathrm{H}), 3.88$ (s, 3H), 2.09-2.00 (m, 1H), 1.41 (t, J=7.2 Hz, 3H), 1.18-1.10 (m, 2H), 0.79-0.69 (m, $2 \mathrm{H}) ;{ }^{13} \mathrm{C} \mathrm{NMR}\left(101 \mathrm{MHz}, \mathrm{CDCl}_{3}\right) \delta: 165.1,163.2,158.8,154.6,153.8,127.5,119.2,113.2,112.7,100.6$, 61.9, 55.8, 14.1, 12.2, 6.6. HRMS (ESI) m/z: [M+Na] calcd for $\mathrm{C}_{16} \mathrm{H}_{16} \mathrm{O}_{5} \mathrm{Na}$ 311.0890; Found 311.0895.

\section{Ethyl 4-cyclopropyl-6-methoxy-2-oxo-2H-chromene-3-carboxylate (4n)}<smiles>CCOC(=O)c1c(C2CC2)c2cc(OC)ccc2oc1=O</smiles>

Prepared according to the general procedure from cyclopropyl(2-hydroxy-5methoxyphenyl)methanone ( $960 \mathrm{mg}, 5.0 \mathrm{mmol}$ ), to provide the title compound as light yellow solid (eluent: petroleum ether/ethyl acetate $=10: 1$ to $4: 1$ ), m.p. $67-68{ }^{\circ} \mathrm{C} .8 \mathrm{~h}, 0.69 \mathrm{~g}, 48 \%$ yield, $R_{\mathrm{f}}=0.4$ (petroleum ether/ethyl acetate $=2: 1$ ). ${ }^{1} \mathrm{H}$ NMR (400 MHz, $\left.\mathrm{CDCl}_{3}\right) \delta: 7.47(\mathrm{~d}, J=2.8 \mathrm{~Hz}, 1 \mathrm{H}), 7.18(\mathrm{~d}, J=8.8 \mathrm{~Hz}, 1 \mathrm{H}), 7.11(\mathrm{dd}, J=8.8,2.8$ $\mathrm{Hz}, 1 \mathrm{H}), 4.44$ (q, J = 7.2 Hz, 2H), $3.87(\mathrm{~s}, 3 \mathrm{H}), 2.11-2.00(\mathrm{~m}, 1 \mathrm{H}), 1.43(\mathrm{t}, J=7.2 \mathrm{~Hz}, 3 \mathrm{H}), 1.21-1.14$ $(\mathrm{m}, 2 \mathrm{H}), 0.81-0.74(\mathrm{~m}, 2 \mathrm{H}) ;{ }^{13} \mathrm{C} \mathrm{NMR}\left(101 \mathrm{MHz}, \mathrm{CDCl}_{3}\right) \delta: 164.6,158.2,155.9,152.6,146.8,122.4$, 120.0, 119.5, 117.5, 108.9, 61.8, 55.6, 14.0, 12.0, 6.5. HRMS (ESI) m/z: $[\mathrm{M}+\mathrm{Na}]^{+}$calcd for $\mathrm{C}_{16} \mathrm{H}_{16} \mathrm{O}_{5} \mathrm{Na}$ 311.0890 ; Found 311.0895 .

\section{Ethyl 2-oxo-4-phenyl-2H-chromene-3-carboxylate (4o) ${ }^{11}$}<smiles>CCOC(=O)c1c(-c2ccccc2)c2ccccc2oc1=O</smiles>

Prepared according to the general procedure from (2hydroxyphenyl)(phenyl)methanone (990 $\mathrm{mg}, 5.0 \mathrm{mmol}$ ), to provide the title compound as white solid (eluent: petroleum ether/ethyl acetate $=10: 1$ to 4:1), m.p. $110-111^{\circ} \mathrm{C}, 8 \mathrm{~h}, 1.01 \mathrm{~g}, 69 \%$ yield, $R_{\mathrm{f}}=0.7$ (petroleum ether/ethyl acetate $\left.=2: 1\right) .{ }^{1} \mathrm{H}$ NMR $(400 \mathrm{MHz}$, $\left.\mathrm{CDCl}_{3}\right) \delta: 7.59$ (ddt, $\left.J=8.4,6.8,1.5 \mathrm{~Hz}, 1 \mathrm{H}\right), 7.56-7.48(\mathrm{~m}, 3 \mathrm{H}), 7.45-7.34(\mathrm{~m}, 3 \mathrm{H}), 7.29-7.20$ (m, 2H), $4.08(\mathrm{q}, J=7.2 \mathrm{~Hz}, 2 \mathrm{H}), 0.97(\mathrm{t}, J=7.2 \mathrm{~Hz}, 3 \mathrm{H})$ (the spectra are in accordance with those of the compound reported in the literature).

\section{(B) Asymmetric Hydrogenation of 3-Ethoxycarbonyl Quinolin-2-ones and Coumarins}

\section{Asymmetric hydrogenation of 4-substituted 3-ethoxycarbonyl quinolinones 2}
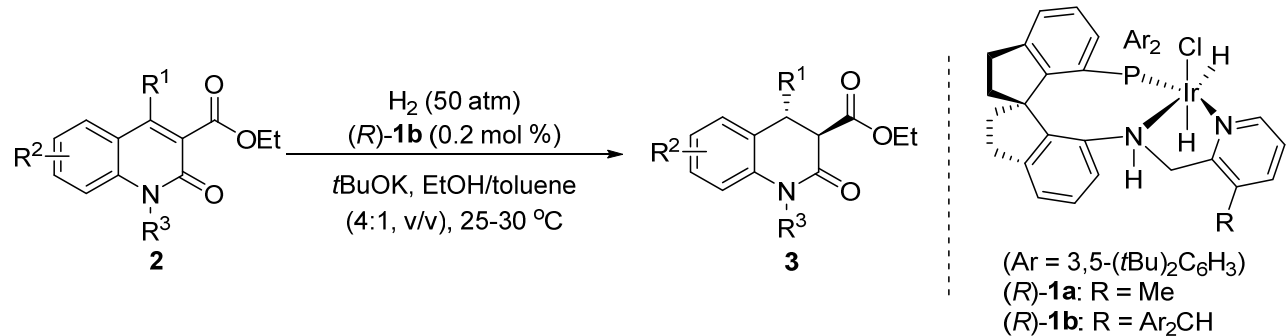

General procedure for $\mathbf{S} / \mathbf{C}=\mathbf{5 0 0}$ : To a $20 \mathrm{~mL}$ hydrogenation vessel was added the 4-substituted 3ethoxycarbonyl quinolinones $\mathbf{2}(1.0 \mathrm{mmol})$, a solution of iridium catalyst $(R)-\mathbf{1 b}(2.7 \mathrm{mg}, 0.002 \mathrm{mmol})$ or $(R)-1 \mathbf{a}(2.0 \mathrm{mg}, 0.002 \mathrm{mmol})$ in $\mathrm{EtOH}(1 \mathrm{~mL})$, and a solution of $t \mathrm{BuOK}(44.8 \mathrm{mg}, 0.4 \mathrm{mmol})$ in EtOH $(1.0 \mathrm{~mL})$ and toluene $(0.5 \mathrm{~mL})$ as co-solvent under nitrogen atmosphere. The autoclave was purged with 
hydrogen by pressurizing to $5 \mathrm{~atm}$ and releasing the pressure. This procedure was repeated three times and then pressurized to $50 \mathrm{~atm}$ of $\mathrm{H}_{2}$. The reaction mixture was stirred at room temperature $\left(25-30^{\circ} \mathrm{C}\right)$ until no obvious hydrogen pressure drop was observed. After releasing the hydrogen pressure, the reaction mixture was filtered through a short silica gel column, and the filtrate was concentrated in vacuo. The residue was purified by chromatography on silica gel with petroleum ether/ethyl acetate (10:1 to 5:1) as eluent to afford the corresponding product $\mathbf{3}$ as a colorless oil or white solid. The enantiomeric excess of the product 3 was determined by chiral HPLC.

(+)-Ethyl 1,4-dimethyl-2-oxo-1,2,3,4-tetrahydroquinoline-3-carboxylate (3a)<smiles>CCOC(=O)C1C(=O)N([N+](=O)[O-])c2ccccc2C1C</smiles>

Prepared according to the general procedure from $2 \mathbf{a}(245 \mathrm{mg}, 1.0 \mathrm{mmol})$, to provide the title compound as colorless oil (eluent: petroleum ether/ethyl acetate $=10: 1$ to $5: 1), 22 \mathrm{~h}, 235 \mathrm{mg}, 95 \%$ yield, $R_{\mathrm{f}}=0.8$ (petroleum ether/ethyl acetate $=2: 1$ ), 99\% ee, $7: 1$ trans/cis. $[\alpha]_{\mathrm{D}}^{24}+47.2\left(c 1.0, \mathrm{CHCl}_{3}\right) .{ }^{1} \mathrm{H}$ NMR $\left(400 \mathrm{MHz}, \mathrm{CDCl}_{3}\right) \delta: 7.26(\mathrm{td}, J=7.8,1.6 \mathrm{~Hz}$, $1 \mathrm{H}), 7.24-7.14(\mathrm{~m}, 1 \mathrm{H}), 7.03(\mathrm{~d}, J=23.4 \mathrm{~Hz}, 2 \mathrm{H}), 4.22-3.99(\mathrm{~m}, 2 \mathrm{H}), 3.66-3.28(\mathrm{~m}, 5 \mathrm{H}), 1.30(\mathrm{~d}, J=$ $6.7 \mathrm{~Hz}, 3 \mathrm{H}), 1.14(\mathrm{t}, J=7.1 \mathrm{~Hz}, 3 \mathrm{H}) ;{ }^{13} \mathrm{C}$ NMR $\left(101 \mathrm{MHz}, \mathrm{CDCl}_{3}\right) \delta: 169.1,166.0,138.7,128.6,127.8$, 126.7, 123.4, 114.9, 61.3, 55.1, 33.9, 29.7, 18.5, 14.0. HRMS (ESI) m/z: $[\mathrm{M}+\mathrm{H}]^{+}$calcd for $\mathrm{C}_{14} \mathrm{H}_{18} \mathrm{NO}_{3}$ 248.1281; Found 248.1286. HPLC analysis (Chiralpak OD-H column, hexane: isopropanol = 90:10; flow rate $=1 \mathrm{~mL} / \mathrm{min}$; UV detection at $254 \mathrm{~nm}$ ): $t_{R}($ minor $)=6.8 \mathrm{~min} ; t_{R}$ (major) $=7.7 \mathrm{~min}$ (ee value was determined by decarboxylation to 1-methyl-4-phenyl-3,4-dihydroquinolin-2(1H)-one).

\section{(-)-Ethyl 4-ethyl-1-methyl-2-oxo-1,2,3,4-tetrahydroquinoline-3-carboxylate (3b)}

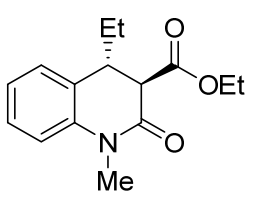

Prepared according to the general procedure from $2 \mathbf{b}(259 \mathrm{mg}, 1.0 \mathrm{mmol})$, to provide the title compound as colorless oil (eluent: petroleum ether/ethyl acetate $=10: 1$ to 5:1), $22 \mathrm{~h}, 245 \mathrm{mg}, 94 \%$ yield, $R_{\mathrm{f}}=0.4$ (petroleum ether/ethyl acetate $=5: 1$ ), $99 \%$ ee, 11:1 trans/cis. $[\alpha]_{\mathrm{D}}^{25}-5.6\left(c 1.0, \mathrm{CHCl}_{3}\right) .{ }^{1} \mathrm{H} \mathrm{NMR}\left(400 \mathrm{MHz}, \mathrm{CDCl}_{3}\right) \delta: 7.36-$ $7.22(\mathrm{~m}, 1 \mathrm{H}), 7.21-7.10(\mathrm{~m}, 1 \mathrm{H}), 7.09-6.74(\mathrm{~m}, 2 \mathrm{H}), 4.21-3.83(\mathrm{~m}, 2 \mathrm{H}), 3.62(\mathrm{~d}, J=2.4 \mathrm{~Hz}, 1 \mathrm{H}), 3.41$ (s, 3H), 3.14 (ddd, $J=8.8,6.8,2.6 \mathrm{~Hz}, 1 \mathrm{H}), 2.10-1.41(\mathrm{~m}, 2 \mathrm{H}), 1.03$ (t, $J=7.2 \mathrm{~Hz}, 3 \mathrm{H}), 0.94$ (t, $J=7.4$ $\mathrm{Hz}, 3 \mathrm{H}) ;{ }^{13} \mathrm{C}$ NMR (101 MHz, $\left.\mathrm{CDCl}_{3}\right) \delta: 169.1,165.8,138.9,128.7,127.9,127.0,123.0,115.0,61.4$, 53.3, 42.0, 29.7, 26.6, 13.9, 11.4; HRMS (ESI) m/z: $[\mathrm{M}+\mathrm{Na}]^{+}$calcd for $\mathrm{C}_{15} \mathrm{H}_{19} \mathrm{NO}_{3} \mathrm{Na}$ 284.1257; Found 284.1262. HPLC analysis (Chiralpak AD-H column, hexane: isopropanol $=95: 5$; flow rate $=1 \mathrm{~mL} / \mathrm{min}$; UV detection at $210 \mathrm{~nm}$ ): $t_{1}=9.1 \mathrm{~min}$ (minor); $t_{2}=9.9 \mathrm{~min}$ (major); $t_{3}=10.9 \mathrm{~min}$ (minor); $t_{4}=11.5 \mathrm{~min}$ (minor).

(+)-Ethyl 1-methyl-2-oxo-4-propyl-1,2,3,4-tetrahydroquinoline-3-carboxylate (3c)

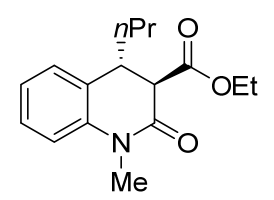

Prepared according to the general procedure from $2 \mathrm{c}$ (273 $\mathrm{mg}, 1.0 \mathrm{mmol})$, to provide the title compound as colorless oil (eluent: petroleum ether/ethyl acetate $=10: 1$ to 5:1), $22 \mathrm{~h}, 258 \mathrm{mg}, 94 \%$ yield, $R_{\mathrm{f}}=0.5$ (petroleum ether/ethyl acetate $=5: 1$ ), $99 \%$ ee, $12: 1$ trans/cis. $[\alpha]_{\mathrm{D}}^{25}+1.4\left(c 1.0, \mathrm{CHCl}_{3}\right)$. ${ }^{1} \mathrm{H} \mathrm{NMR}\left(400 \mathrm{MHz}, \mathrm{CDCl}_{3}\right) \delta: 7.34$ $7.20(\mathrm{~m}, 1 \mathrm{H}), 7.21-7.09(\mathrm{~m}, 1 \mathrm{H}), 7.09-6.77(\mathrm{~m}, 2 \mathrm{H}), 4.25-3.85(\mathrm{~m}, 2 \mathrm{H}), 3.60(\mathrm{~d}, J=2.0 \mathrm{~Hz}, 1 \mathrm{H}), 3.42$ (s, 3H), 3.29-3.03 (m, 1H), 1.74-1.45 (m, 2H), 1.45-1.22 (m, 2H), 1.03 (t, $J=7.0 \mathrm{~Hz}, 3 \mathrm{H}), 0.90$ (t, $J=$ $7.2 \mathrm{~Hz}, 3 \mathrm{H}) ;{ }^{13} \mathrm{C} \mathrm{NMR}\left(101 \mathrm{MHz}, \mathrm{CDCl}_{3}\right) \delta: 169.0,165.7,138.8,128.5,127.8,127.2,123.0,115.0,61.3$, 53.4, 40.2, 35.7, 29.6, 20.0, 13.8, 13.8; HRMS (ESI) m/z: [M+H] $]^{+}$calcd for $\mathrm{C}_{16} \mathrm{H}_{22} \mathrm{NO}_{3} 276.1594$; Found 276.1599. HPLC analysis (Chiralpak AS-H column, hexane: isopropanol $=90: 10$; flow rate $=1 \mathrm{~mL} / \mathrm{min}$; 
UV detection at $210 \mathrm{~nm}): t_{1}=8.7 \min ($ major $) ; t_{2}=13.5 \mathrm{~min}($ minor $) ; t_{3}=15.3 \mathrm{~min}(\operatorname{minor}) ; t_{4}=16.6$ $\min$ (minor).

\section{(+)-Ethyl 4-isobutyl-1-methyl-2-oxo-1,2,3,4-tetrahydroquinoline-3-carboxylate (3d)}

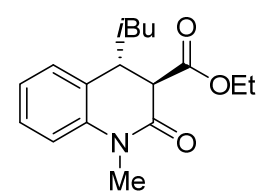

Prepared according to the general procedure from $\mathbf{2 d}(287 \mathrm{mg}, 1.0 \mathrm{mmol})$, to provide the title compound as colorless oil (eluent: petroleum ether/ethyl acetate $=10: 1$ to $5: 1$ ), $24 \mathrm{~h}, 271 \mathrm{mg}, 94 \%$ yield, $R_{\mathrm{f}}=0.7$ (petroleum ether/ethyl acetate $=2: 1$ ), $99 \%$ ee, $11: 1$ trans/cis. $[\alpha]_{\mathrm{D}}^{24}+12.6\left(\right.$ c $\left.1.0, \mathrm{CHCl}_{3}\right) .{ }^{1} \mathrm{H}$ NMR $\left(400 \mathrm{MHz}, \mathrm{CDCl}_{3}\right) \delta: 7.25(\mathrm{t}$, $J=7.8 \mathrm{~Hz}, 1 \mathrm{H}), 7.13(\mathrm{~d}, J=7.5 \mathrm{~Hz}, 1 \mathrm{H}), 7.08-6.89(\mathrm{~m}, 2 \mathrm{H}), 4.25-3.84(\mathrm{~m}, 2 \mathrm{H}), 3.57$ (d, $J=4.4 \mathrm{~Hz}$, $1 \mathrm{H}), 3.42$ (s, 3H), 3.33 (t, $J=7.8 \mathrm{~Hz}, 1 \mathrm{H}), 1.86-1.50(\mathrm{~m}, 1 \mathrm{H}), 1.39$ (t, $J=7.4 \mathrm{~Hz}, 2 \mathrm{H}), 1.18-0.94(\mathrm{~m}$, $6 \mathrm{H}), 0.90(\mathrm{~d}, J=6.8 \mathrm{~Hz}, 3 \mathrm{H}) ;{ }^{13} \mathrm{C} \mathrm{NMR}\left(101 \mathrm{MHz}, \mathrm{CDCl}_{3}\right) \delta: 169.0,165.7,138.9,128.3,127.8,127.6$, 123.1, 115.1, 61.4, 53.3, 42.7, 38.3, 29.7, 24.9, 22.5, 13.9; HRMS (ESI) m/z: $[\mathrm{M}+\mathrm{H}]^{+}$calcd for $\mathrm{C}_{17} \mathrm{H}_{24} \mathrm{NO}_{3}$ 290.1751; Found 290.1754. HPLC analysis (Chiralpak AD-H column, hexane: isopropanol $=95: 5$; flow rate $=1 \mathrm{~mL} / \mathrm{min}$; UV detection at $210 \mathrm{~nm}$ ): $t_{\mathrm{R}}=5.7 \mathrm{~min}$ (major); $t_{\mathrm{R}}=6.0$ min (minor) (ee value was tested after decarboxylation of its 3-position ester group).

\section{(+)-Ethyl 4-benzyl-1-methyl-2-oxo-1,2,3,4-tetrahydroquinoline-3-carboxylate (3e)}

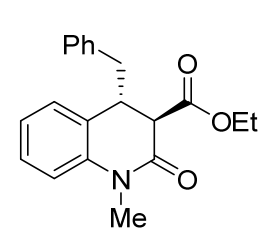

Prepared according to the general procedure from $2 \mathrm{e}(321 \mathrm{mg}, 1.0 \mathrm{mmol})$, to provide the title compound as a white solid (eluent: petroleum ether/ethyl acetate $=10: 1$ to 5:1), m.p. $123-124{ }^{\circ} \mathrm{C}, 48 \mathrm{~h}, 306 \mathrm{mg}, 95 \%$ yield, $R_{\mathrm{f}}=0.5$ (petroleum ether/ethyl acetate $=2: 1), 99 \%$ ee, $18: 1$ trans $/$ cis. $[\alpha]_{\mathrm{D}}^{25}+76.2\left(\right.$ c 1.0, $\left.\mathrm{CHCl}_{3}\right) .{ }^{1} \mathrm{H} \mathrm{NMR}(400$ $\left.\mathrm{MHz} \mathrm{CDCl}_{3}\right) \delta:$ 7.34-7.13 (m, 4H), 7.12-6.85 (m, 5H), 4.06-3.85 (m, 2H), 3.60 (d, $J=1.7 \mathrm{~Hz}, 1 \mathrm{H}), 3.51(\mathrm{t}, J=8.5 \mathrm{~Hz}, 1 \mathrm{H}), 3.39(\mathrm{~s}, 3 \mathrm{H}), 2.92-2.80(\mathrm{~m}, 1 \mathrm{H}), 2.80-2.57(\mathrm{~m}, 1 \mathrm{H}), 0.97(\mathrm{t}, J$ $=7.1 \mathrm{~Hz}, 3 \mathrm{H}) ;{ }^{13} \mathrm{C} \mathrm{NMR}\left(101 \mathrm{MHz}, \mathrm{CDCl}_{3}\right) \delta: 168.9,165.2,139.0,137.8,129.4,128.7,128.5,128.1$, 126.7, 126.6, 123.2, 115.0, 61.4, 52.3, 42.5, 40.5, 29.7, 13.9; HRMS (ESI) m/z: $[\mathrm{M}+\mathrm{H}]^{+}$calcd for $\mathrm{C}_{20} \mathrm{H}_{22} \mathrm{NO}_{3}$ 324.1594; Found 324.1593. HPLC analysis (Chiralpak OD-H column, hexane: isopropanol $=90: 10$; flow rate $=1 \mathrm{~mL} / \mathrm{min}$; UV detection at $210 \mathrm{~nm}): t_{R}($ minor $)=9.6 \mathrm{~min} ; t_{R}$ (major) $=10.7 \mathrm{~min}($ ee value was tested after decarboxylation of its 3-position ester group).

\section{(+)-Ethyl 1-methyl-2-oxo-4-phenethyl-1,2,3,4-tetrahydroquinoline-3-carboxylate (3f)}

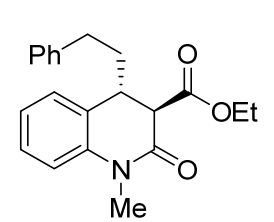

Prepared according to the general procedure from $\mathbf{2 f}(335 \mathrm{mg}, 1.0 \mathrm{mmol})$, to provide the title compound as a colorless oil (eluent: petroleum ether/ethyl acetate $=10: 1$ to $5: 1$ ), $24 \mathrm{~h}, 320 \mathrm{mg}, 95 \%$ yield, $R_{\mathrm{f}}=0.6$ (petroleum ether/ethyl acetate $=2: 1$ ), $99 \%$ ee, $11: 1$ trans/cis. $[\alpha]_{\mathrm{D}}^{25}+33.6\left(c_{1} 1.0, \mathrm{CHCl}_{3}\right) .{ }^{1} \mathrm{H} \mathrm{NMR}\left(400 \mathrm{MHz}, \mathrm{CDCl}_{3}\right) \delta: 7.40$ $7.31(\mathrm{~m}, 3 \mathrm{H}), 7.31-7.18(\mathrm{~m}, 4 \mathrm{H}), 7.12(\mathrm{t}, J=7.3 \mathrm{~Hz}, 2 \mathrm{H}), 4.11(\mathrm{~d}, J=39.9 \mathrm{~Hz}, 2 \mathrm{H})$, $3.79(\mathrm{~d}, J=2.3 \mathrm{~Hz}, 1 \mathrm{H}), 3.49$ (s, 3H), 3.41 (t, $J=8.4 \mathrm{~Hz}, 1 \mathrm{H}), 2.91-2.47(\mathrm{~m}, 2 \mathrm{H}), 2.33-1.78(\mathrm{~m}, 2 \mathrm{H})$, $1.11(\mathrm{t}, J=7.1 \mathrm{~Hz}, 3 \mathrm{H}) ;{ }^{13} \mathrm{C}$ NMR $\left(101 \mathrm{MHz} \mathrm{CDCl}_{3}\right) \delta: 168.9,165.6,141.0,139.0,128.7,128.5,128.3$, 128.0, 126.9, 126.1, 123.2, 115.1, 61.5, 53.4, 40.0, 35.1, 32.9, 29.8, 13.9; HRMS (ESI) m/z: [M+H] ${ }^{+}$ calcd for $\mathrm{C}_{21} \mathrm{H}_{24} \mathrm{NO}_{3}$ 338.1751; Found 338.1756. HPLC analysis (Chiralpak AD-H column, hexane: isopropanol $=90: 10$; flow rate $=1 \mathrm{~mL} / \mathrm{min}$; UV detection at $210 \mathrm{~nm}): t_{R}($ minor $)=6.5 \mathrm{~min} ; t_{R}($ major $)=$ $7.0 \mathrm{~min}$ (ee value was tested after decarboxylation of its 3-position ester group).

(+)-Ethyl 1-methyl-2-oxo-4-pentyl-1,2,3,4-tetrahydroquinoline-3-carboxylate (3g) 


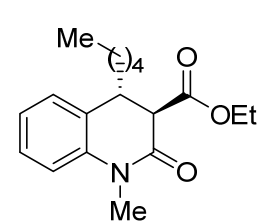

Prepared according to the general procedure from $\mathbf{2 g}(301 \mathrm{mg}, 1.0 \mathrm{mmol})$, to provide the title compound as a colorless oil (eluent: petroleum ether/ethyl acetate $=10: 1$ to 5:1), $24 \mathrm{~h}, 288 \mathrm{mg}, 95 \%$ yield, $R_{\mathrm{f}}=0.6$ (petroleum ether/ethyl acetate $=2: 1$ ), $99 \%$ ee, $14: 1$ trans/cis. $[\alpha]_{\mathrm{D}}^{26}+11.8\left(\right.$ c $\left.1.0,^{\mathrm{CHCl}} \mathrm{CH}_{3}\right) .{ }^{1} \mathrm{H} \mathrm{NMR}\left(400 \mathrm{MHz}, \mathrm{CDCl}_{3}\right) \delta: 7.30$ $7.23(\mathrm{~m}, 1 \mathrm{H}), 7.13$ (d, $J=6.7 \mathrm{~Hz}, 1 \mathrm{H}), 7.02$ (t, $J=7.3 \mathrm{~Hz}, 2 \mathrm{H}), 4.17-3.92(\mathrm{~m}, 2 \mathrm{H})$, $3.60(\mathrm{~d}, J=2.3 \mathrm{~Hz}, 1 \mathrm{H}), 3.42(\mathrm{~s}, 3 \mathrm{H}), 3.22(\mathrm{t}, J=7.5 \mathrm{~Hz}, 1 \mathrm{H}), 1.60-1.46(\mathrm{~m}, 2 \mathrm{H}), 1.44-1.20(\mathrm{~m}, 6 \mathrm{H})$, $1.03(\mathrm{t}, J=7.1 \mathrm{~Hz}, 3 \mathrm{H}), 0.86(\mathrm{t}, J=6.5 \mathrm{~Hz}, 3 \mathrm{H}) ;{ }^{13} \mathrm{C} \mathrm{NMR}\left(101 \mathrm{MHz}, \mathrm{CDCl}_{3}\right) \delta: 169.1,165.8,138.9$, 128.6, 127.8, 127.4, 123.1, 115.0, 61.4, 53.5, 40.5, 33.6, 31.6, 29.7, 26.5, 22.5, 14.0, 13.9; HRMS (ESI) $\mathrm{m} / \mathrm{z}:[\mathrm{M}+\mathrm{Na}]^{+}$calcd for $\mathrm{C}_{18} \mathrm{H}_{25} \mathrm{NO}_{3} \mathrm{Na}$ 326.1727; Found 326.1730. HPLC analysis (Chiralpak OD-H column, hexane: isopropanol $=90: 10$; flow rate $=1 \mathrm{~mL} / \mathrm{min}$; UV detection at $210 \mathrm{~nm}): t_{R}($ major $)=5.5$ $\min ; t_{R}($ minor $)=6.0 \mathrm{~min}$ (ee value was tested after decarboxylation of its 3-position ester group).

\section{(+)-Ethyl 4-cyclopropyl-1-methyl-2-oxo-1,2,3,4-tetrahydroquinoline-3-carboxylate (3h)}

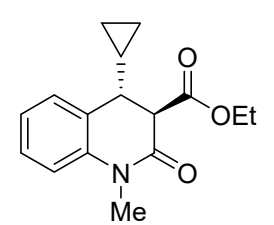

Prepared according to the general procedure from $\mathbf{2 h}(271 \mathrm{mg}, 1.0 \mathrm{mmol})$, to provide the title compound as colorless oil (eluent: petroleum ether/ethyl acetate $=10: 1$ to $5: 1), 48 \mathrm{~h}, 144 \mathrm{mg}, 53 \%$ yield $((R)-1 \mathrm{a}, \mathrm{S} / \mathrm{C}=500), R_{\mathrm{f}}=0.6$ (petroleum ether/ethyl acetate $=2: 1), 94 \%$ ee, 10:1 trans/cis. $[\alpha]_{\mathrm{D}}^{27}+82.2\left(\right.$ c 1.0, MeOH). ${ }^{1} \mathrm{H}$ NMR $(400$ $\mathrm{MHz}_{\mathrm{CDCl}}$ ) $\delta:$ 7.35-7.25 (m, 2H), 7.14-6.98 (m, 2H), 4.17-3.97 (m, 2H), $3.68(\mathrm{~d}$, $J=5.2 \mathrm{~Hz}, 1 \mathrm{H}), 3.42(\mathrm{~s}, 3 \mathrm{H}), 2.62-2.34(\mathrm{~m}, 1 \mathrm{H}), 1.11(\mathrm{t}, J=7.1 \mathrm{~Hz}, 3 \mathrm{H}), 0.97-0.85(\mathrm{~m}, 1 \mathrm{H}), 0.58(\mathrm{~d}, J$ $=8.0 \mathrm{~Hz}, 2 \mathrm{H}), 0.32(\mathrm{~d}, J=4.5 \mathrm{~Hz}, 2 \mathrm{H}) ;{ }^{13} \mathrm{C} \mathrm{NMR}\left(101 \mathrm{MHz}, \mathrm{CDCl}_{3}\right) \delta: 169.1,166.2,139.0,128.1$, 127.9, 126.9, 123.3, 114.9, 61.4, 54.5, 44.3, 29.8, 14.7, 14.0, 4.5, 3.8; HRMS (ESI) m/z: [M+Na $]^{+}$calcd for $\mathrm{C}_{16} \mathrm{H}_{19} \mathrm{NO}_{3} \mathrm{Na}$ 296.1257; Found 296.1260. HPLC analysis (Chiralpak AS-H column, hexane: isopropanol $=90: 10$; flow rate $=1 \mathrm{~mL} / \mathrm{min}$; $U V$ detection at $210 \mathrm{~nm}$ ): $t_{1}=13.0 \mathrm{~min}$ (major); $t_{2}=15.1$ $\min$ (minor); $t_{3}=17.4 \min$ (minor); $t_{4}=19.0 \mathrm{~min}$ (minor).

\section{(-)-(3S,4R)-Ethyl 1-methyl-2-oxo-4-phenyl-1,2,3,4-tetrahydroquinoline-3-carboxylate ((3S,4R)-3i)}<smiles>CCOC(=O)[C@H]1C(=O)N(C)c2ccccc2[C@@H]1c1ccccc1</smiles>

Prepared according to the general procedure from $2 \mathbf{i}(307 \mathrm{mg}, 1.0 \mathrm{mmol})$, to provide the title compound as colorless oil (eluent: petroleum ether/ethyl acetate $=10: 1$ to $5: 1), 48 \mathrm{~h}, 102 \mathrm{mg}, 33 \%$ yield $((R)-1 \mathrm{a}, \mathrm{S} / \mathrm{C}=500), R_{\mathrm{f}}=0.5$ (petroleum ether/ethyl acetate $=2: 1), 75 \%$ ee, $>20: 1$ trans $/$ cis. $[\alpha]_{\mathrm{D}}^{27}-81.0($ c $1.0, \mathrm{MeOH})[$ the absolute configuration was determined by converting it to 1-methyl-4-phenyl-3,4-dihydroquinolin-2(1H)-one and comparing its optical rotation that reported for $(S)$-isomer. $[\alpha]_{\mathrm{D}}^{26}-34.9\left(c 0.24, \mathrm{CHCl}_{3}\right)$, lit. $^{12}[\alpha]_{\mathrm{D}}^{20}+37.4$ (c $\left.0.24, \mathrm{CHCl}_{3}\right), 95 \%$ ee for (S)-isomer]. ${ }^{1} \mathrm{H} \mathrm{NMR}\left(400 \mathrm{MHz}, \mathrm{CDCl}_{3}\right) \delta: 7.41-7.22(\mathrm{~m}, 4 \mathrm{H}), 7.17(\mathrm{~d}, J=$ $7.4 \mathrm{~Hz}, 2 \mathrm{H}), 7.06$ (d, $J=8.1 \mathrm{~Hz}, 1 \mathrm{H}), 6.98(\mathrm{t}, J=7.4 \mathrm{~Hz}, 1 \mathrm{H}), 6.85$ (d, $J=7.4 \mathrm{~Hz}, 1 \mathrm{H}), 4.61(\mathrm{~d}, J=9.6$ $\mathrm{Hz}, 1 \mathrm{H}), 4.15-3.97(\mathrm{~m}, 2 \mathrm{H}), 3.90(\mathrm{~d}, J=9.6 \mathrm{~Hz}, 1 \mathrm{H}), 3.42(\mathrm{~s}, 3 \mathrm{H}), 1.03(\mathrm{td}, J=7.1,2.0 \mathrm{~Hz}, 3 \mathrm{H}) ;{ }^{13} \mathrm{C}$

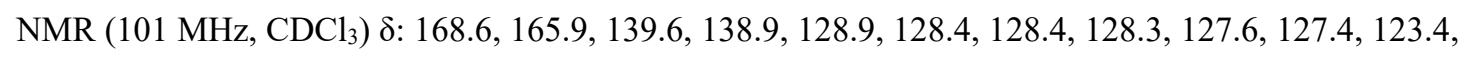
115.0, 61.4, 55.1, 44.9, 29.9, 13.9; HRMS (ESI) m/z: $[\mathrm{M}+\mathrm{H}]^{+}$calcd for $\mathrm{C}_{19} \mathrm{H}_{20} \mathrm{NO}_{3} 310.1438$; Found 310.1436. HPLC analysis (Chiralpak OD-H column, hexane: isopropanol = 90:10; flow rate $=1 \mathrm{~mL} / \mathrm{min}$; $\mathrm{UV}$ detection at $254 \mathrm{~nm}): t_{R}($ minor $)=18.6 \mathrm{~min} ; t_{R}($ major $)=21.6 \mathrm{~min}$.

(+)-Ethyl 6-bromo-1,4-dimethyl-2-oxo-1,2,3,4-tetrahydroquinoline-3-carboxylate (3j) 
<smiles>CCOC(=O)[C@H]1C(=O)N(C)c2ccc(Br)cc2C1C</smiles>

Prepared according to the general procedure from $\mathbf{2 j}$ (323 $\mathrm{mg}, 1.0 \mathrm{mmol}$ ), to provide the title compound as colorless oil (eluent: petroleum ether/ethyl acetate $=10: 1$ to $5: 1$ ), $24 \mathrm{~h}, 305 \mathrm{mg}, 94 \%$ yield, $R_{\mathrm{f}}=0.7$ (petroleum ether/ethyl acetate $=2: 1), 98 \%$ ee, $7: 1$ trans/cis. $[\alpha]_{\mathrm{D}}^{28}+46.2\left(\right.$ c 1.0, $\left.\mathrm{CHCl}_{3}\right) .{ }^{1} \mathrm{H} \mathrm{NMR}(400$ $(\mathrm{m}, 2 \mathrm{H}), 3.48-3.30(\mathrm{~m}, 5 \mathrm{H}), 1.45-1.26(\mathrm{~m}, 3 \mathrm{H}), 1.18(\mathrm{t}, J=7.2 \mathrm{~Hz}, 3 \mathrm{H}) ;{ }^{13} \mathrm{C}$ NMR $\left(101 \mathrm{MHz}, \mathrm{CDCl}_{3}\right)$ $\delta: 168.6,165.5,138.1,130.8,130.6,129.6,116.6,116.1,61.5,54.7,33.8,29.8,18.5,14.0$; HRMS (ESI) $\mathrm{m} / \mathrm{z}:[\mathrm{M}+\mathrm{H}]^{+}$calcd for $\mathrm{C}_{14} \mathrm{H}_{17} \mathrm{BrNO}_{3}$ 326.0386; Found 326.0387. HPLC analysis (Chiralpak AS-H column, hexane: isopropanol $=90: 10$; flow rate $=1 \mathrm{~mL} / \mathrm{min}$; UV detection at $254 \mathrm{~nm}): t_{1}=11.4 \mathrm{~min}$ (major); $t_{2}=13.8 \mathrm{~min}$ (minor); $t_{3}=16.9 \mathrm{~min}$ (minor); $t_{4}=24.7 \mathrm{~min}$ (minor).

\section{(-)-Ethyl 6-methoxy-1,4-dimethyl-2-oxo-1,2,3,4-tetrahydroquinoline-3-carboxylate (3k)}<smiles>CCOC(=O)C1C(=O)N(C)c2ccc(OC)cc2C1C</smiles>

Prepared according to the general procedure from $2 \mathbf{k}(275 \mathrm{mg}, 1.0 \mathrm{mmol})$, to provide the title compound as colorless oil (eluent: petroleum ether/ethyl acetate $=10: 1$ to $5: 1), 48 \mathrm{~h}, 220 \mathrm{mg}, 80 \%$ yield $\left(\mathrm{S} / \mathrm{C}=250,0.2 \mathrm{M}, 50{ }^{\circ} \mathrm{C}\right.$ in an oil bath), $R_{\mathrm{f}}=0.6$ (petroleum ether/ethyl acetate $=2: 1$ ), $98 \%$ ee, $8: 1$ trans/cis. $[\alpha]_{\mathrm{D}}^{27}-52.4\left(\right.$ c $\left.0.5, \mathrm{CHCl}_{3}\right) .{ }^{1} \mathrm{H} \mathrm{NMR}\left(400 \mathrm{MHz}, \mathrm{CDCl}_{3}\right) \delta: 6.94(\mathrm{~d}, J=9.2 \mathrm{~Hz}, 1 \mathrm{H}), 6.83-6.67$ $(\mathrm{m}, 2 \mathrm{H}), 4.23-3.97(\mathrm{~m}, 2 \mathrm{H}), 3.81(\mathrm{~s}, 3 \mathrm{H}), 3.65-3.27(\mathrm{~m}, 5 \mathrm{H}), 1.31(\mathrm{~d}, J=6.4 \mathrm{~Hz}, 3 \mathrm{H}), 1.17(\mathrm{t}, J=7.2$ $\mathrm{Hz}, 3 \mathrm{H}) ;{ }^{13} \mathrm{C} \mathrm{NMR}\left(101 \mathrm{MHz}, \mathrm{CDCl}_{3}\right) \delta: 169.2,165.6,155.8,132.5,130.3,115.9,113.2,112.0,61.4$, 55.5, 55.1, 34.2, 29.9, 18.7, 14.0; HRMS (ESI) m/z: [M+H] ${ }^{+}$calcd for $\mathrm{C}_{15} \mathrm{H}_{20} \mathrm{NO}_{4}$ 278.1387; Found 278.1389. HPLC analysis (Chiralpak OD-3 column, hexane: isopropanol $=90: 10$; flow rate $=1 \mathrm{~mL} / \mathrm{min}$; UV detection at $254 \mathrm{~nm}): t_{R}$ (minor) $=8.3 \mathrm{~min} ; t_{R}$ (major) $=9.4 \mathrm{~min}$ (ee value was tested after decarboxylation of its 3-position ester group).

\section{(+)-Ethyl 1-ethyl-4-methyl-2-oxo-1,2,3,4-tetrahydroquinoline-3-carboxylate (3I)}

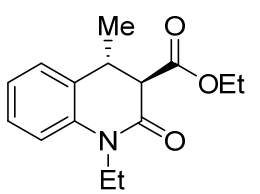

Prepared according to the general procedure from 21 (259 $\mathrm{mg}, 1.0 \mathrm{mmol})$, to provide the title compound as colorless oil (eluent: petroleum ether/ethyl acetate $=10: 1$ to $5: 1), 24 \mathrm{~h}, 242 \mathrm{mg}, 93 \%$ yield $\left(50^{\circ} \mathrm{C}\right), R_{\mathrm{f}}=0.6$ (petroleum ether/ethyl acetate $\left.=2: 1\right)$, $99 \%$ ee, 8:1 trans/cis. $[\alpha]_{\mathrm{D}}^{28}+25.6\left(\right.$ c $\left.0.1, \mathrm{CHCl}_{3}\right) .{ }^{1} \mathrm{H}$ NMR $\left(400 \mathrm{MHz}, \mathrm{CDCl}_{3}\right) \delta$ : 7.32-7.23 (m, 1H), 7.23-7.15 (m, 1H), 7.10-6.99 (m, 2H), 4.21-4.03 (m, 3H), 4.03-3.88 (m, 1H), 3.64$3.22(\mathrm{~m}, 2 \mathrm{H}), 1.56-1.22(\mathrm{~m}, 6 \mathrm{H}), 1.12(\mathrm{t}, J=7.2 \mathrm{~Hz}, 3 \mathrm{H}) ;{ }^{13} \mathrm{C} \mathrm{NMR}\left(101 \mathrm{MHz}, \mathrm{CDCl}_{3}\right) \delta: 169.0,165.3$, 137.7, 128.8, 127.8, 127.2, 123.2, 114.7, 61.2, 55.0, 37.6, 34.3, 18.9, 13.9, 12.3; HRMS (ESI) m/z: $[\mathrm{M}+\mathrm{H}]^{+}$calcd for $\mathrm{C}_{15} \mathrm{H}_{20} \mathrm{NO}_{3}$ 262.1438; Found 262.1443. HPLC analysis (Chiralpak AS-H column, hexane: isopropanol $=90: 10$; flow rate $=1 \mathrm{~mL} / \mathrm{min}$; $U V$ detection at $210 \mathrm{~nm}): t_{1}=8.8 \mathrm{~min}(\operatorname{minor}) ; t_{2}=$ $9.2 \min$ (major); $t_{3}=10.9 \min$ (minor); $t_{4}=15.5 \min$ (minor).

\section{(+)-Ethyl 1-benzyl-4-methyl-2-oxo-1,2,3,4-tetrahydroquinoline-3-carboxylate (3m)}

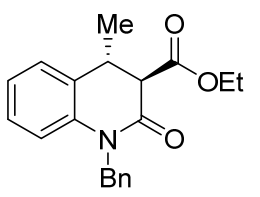

Prepared according to the general procedure from $\mathbf{2 m}(321 \mathrm{mg}, 1.0 \mathrm{mmol})$, to provide the title compound as colorless oil (eluent: petroleum ether/ethyl acetate $=10: 1$ to 5:1), $26 \mathrm{~h}, 303 \mathrm{mg}, 94 \%$ yield, $R_{\mathrm{f}}=0.7$ (petroleum ether/ethyl acetate $=2: 1$ ), $93 \%$ ee, $8: 1$ trans/cis. $[\alpha]_{\mathrm{D}}^{28}+41.0\left(\right.$ c $\left.0.1, \mathrm{CHCl}_{3}\right) .{ }^{1} \mathrm{H} \mathrm{NMR}\left(400 \mathrm{MHz}, \mathrm{CDCl}_{3}\right) \delta: .42-7.27$ (m, 4H), 7.27-7.21 (m, 1H), $7.19(\mathrm{~d}, J=7.4 \mathrm{~Hz}, 1 \mathrm{H}), 7.10(\mathrm{t}, J=7.8 \mathrm{~Hz}, 1 \mathrm{H}), 7.05-6.95$ (m, 1H), 6.88 $(\mathrm{d}, J=7.6 \mathrm{~Hz}, 1 \mathrm{H}), 5.50(\mathrm{~d}, J=16.0 \mathrm{~Hz}, 1 \mathrm{H}), 4.91$ (d, $J=16.4 \mathrm{~Hz}, 1 \mathrm{H}), 4.33-3.86(\mathrm{~m}, 2 \mathrm{H}), 3.58(\mathrm{dd}, J$ $=4.6,2.0 \mathrm{~Hz}, 1 \mathrm{H}), 3.54-3.14(\mathrm{~m}, 1 \mathrm{H}), 1.33(\mathrm{dd}, J=7.2,2.0 \mathrm{~Hz}, 3 \mathrm{H}), 1.15(\mathrm{td}, J=7.2,2.0 \mathrm{~Hz}, 3 \mathrm{H}) ;{ }^{13} \mathrm{C}$ 
NMR (101 MHz, $\left.\mathrm{CDCl}_{3}\right) \delta: 169.1,166.0,138.4,137.0,128.7,128.7,127.9,127.3,127.2,126.5,123.7$, 115.9, 61.6, 55.2, 46.9, 34.8, 19.6, 14.1; HRMS (ESI) m/z: $[\mathrm{M}+\mathrm{H}]^{+}$calcd for $\mathrm{C}_{20} \mathrm{H}_{22} \mathrm{NO}_{3} 324.1594$; Found 324.1599. HPLC analysis (Chiralpak AS-H column, hexane: isopropanol = 90:10; flow rate $=1$ $\mathrm{mL} / \mathrm{min}$; UV detection at $210 \mathrm{~nm}$ ): $t_{1}=11.5 \mathrm{~min}$ (major); $t_{2}=14.0 \mathrm{~min}$ (minor); $t_{3}=22.2 \mathrm{~min}$ (minor); $t_{4}=24.3 \min$ (minor).

\section{(-)-Ethyl 4-methyl-2-oxo-1-phenyl-1,2,3,4-tetrahydroquinoline-3-carboxylate (3n)}

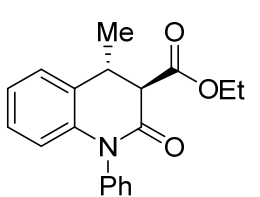

Prepared according to the general procedure from $2 \mathrm{n}(307 \mathrm{mg}, 1.0 \mathrm{mmol})$, to provide the title compound as colorless oil (eluent: petroleum ether/ethyl acetate $=10: 1$ to 5:1), 48 h, $287 \mathrm{mg}, 93 \%$ yield $((R)-1 \mathrm{a}, \mathrm{S} / \mathrm{C}=250), R_{\mathrm{f}}=0.6$ (petroleum ether/ethyl acetate $=2: 1), 90 \%$ ee, $12: 1$ trans/cis. $[\alpha]_{\mathrm{D}}^{28}-39.0\left(\right.$ c 1.0, $\left.\mathrm{CHCl}_{3}\right) .{ }^{1} \mathrm{H}$ NMR $(400 \mathrm{MHz}$, $\left.\mathrm{CDCl}_{3}\right) \delta: 7.51(\mathrm{t}, J=7.4 \mathrm{~Hz}, 2 \mathrm{H}), 7.46-7.36(\mathrm{~m}, 1 \mathrm{H}), 7.34-7.17(\mathrm{~m}, 3 \mathrm{H}), 7.09-6.94(\mathrm{~m}, 2 \mathrm{H}), 6.37$ (d, $J$ $=7.6 \mathrm{~Hz}, 1 \mathrm{H}), 4.26-4.01(\mathrm{~m}, 2 \mathrm{H}), 3.83-3.46(\mathrm{~m}, 2 \mathrm{H}), 1.44(\mathrm{~d}, J=5.2 \mathrm{~Hz}, 3 \mathrm{H}), 1.15(\mathrm{t}, J=7.2 \mathrm{~Hz}, 3 \mathrm{H})$; ${ }^{13} \mathrm{C}$ NMR (101 MHz, $\mathrm{CDCl}_{3}$ ) $\delta: 169.0,165.8,139.9,138.1,129.9,128.8,128.4,128.1,127.6,127.2$, 123.6, 117.2, 61.6, 55.41, 35.0, 19.6, 14.1; HRMS (ESI) m/z: $[\mathrm{M}+\mathrm{H}]^{+}$calcd for $\mathrm{C}_{19} \mathrm{H}_{20} \mathrm{NO}_{3} 310.1438$; Found 310.1435. HPLC analysis (Chiralpak AD-H column, hexane: isopropanol = 90:10; flow rate $=1$ $\mathrm{mL} / \mathrm{min}$; UV detection at $210 \mathrm{~nm}$ ): $t_{R}$ (major) $=7.5 \mathrm{~min} ; t_{R}$ (minor) $=8.5 \mathrm{~min}$ (ee value was tested after decarboxylation of its 3-position ester group).

\section{(-)-(3R,4R)-Ethyl 4-ethyl-2-oxo-1-phenyl-1,2,3,4-tetrahydroquinoline-3-carboxylate ((3R,4R)-3o)}<smiles>CCOC(=O)C1C(=O)N(c2ccccc2)c2ccccc2C1CC</smiles>

Prepared according to the general procedure from $20(321 \mathrm{mg}, 1.0 \mathrm{mmol})$, to provide the title compound as a white solid (eluent: petroleum ether/ethyl acetate $=10: 1$ to 7:1), m.p. $90-91{ }^{\circ} \mathrm{C}, 48 \mathrm{~h}, 300 \mathrm{mg}, 93 \%$ yield $((R)-\mathbf{1 a}, \mathrm{S} / \mathrm{C}=250) . R_{\mathrm{f}}=0.8$ (petroleum ether/ethyl acetate $=2: 1), 92 \%$ ee, 18:1 trans $/$ cis. $[\alpha]_{\mathrm{D}}^{28}-83.2(c 1.0$, $\mathrm{MeOH}) .{ }^{1} \mathrm{H}$ NMR (400 MHz, $\left.\mathrm{CDCl}_{3}\right) \delta: 7.52$ (t, $\left.J=7.6 \mathrm{~Hz}, 2 \mathrm{H}\right), 7.43$ (t, $\left.J=7.4 \mathrm{~Hz}, 1 \mathrm{H}\right), 7.34-7.20(\mathrm{~m}$, 2H), 7.17 (dd, $J=7.2,1.6 \mathrm{~Hz}, 1 \mathrm{H})$ ), 7.11-6.94 (m, 2H), $6.37(\mathrm{dd}, J=8.0,1.2 \mathrm{~Hz}, 1 \mathrm{H}), 4.21-3.99(\mathrm{~m}$, 2H), 3.73 (d, $J=2.4 \mathrm{~Hz}, 1 \mathrm{H}), 3.26$ (td, $J=7.2,2.4 \mathrm{~Hz}, 1 \mathrm{H}), 1.89-1.66(\mathrm{~m}, 2 \mathrm{H}), 1.10$ (t, $J=7.2 \mathrm{~Hz}, 3 \mathrm{H})$, 1.04 (t, $J=7.4 \mathrm{~Hz}, 3 \mathrm{H}) ;{ }^{13} \mathrm{C}$ NMR $\left(101 \mathrm{MHz}, \mathrm{CDCl}_{3}\right) \delta: 175.1,173.5,137.9,136.9,130.3,130.1,129.2$, 128.2, 127.9, 126.6, 124.8, 117.8, 49.8, 47.3, 25.0, 22.8, 11.9. HRMS (ESI) m/z: $[\mathrm{M}+\mathrm{H}]^{+}$calcd for $\mathrm{C}_{20} \mathrm{H}_{22} \mathrm{NO}_{3}$ 324.1594; Found 324.1594. HPLC analysis (Chiralpak OD-H column, hexane: isopropanol $=95: 5$; flow rate $=1 \mathrm{~mL} / \mathrm{min}$; UV detection at $210 \mathrm{~nm}): t_{R}$ (major) $=10.1 \mathrm{~min} ; t_{R}($ minor $)=16.1 \mathrm{~min}($ ee value was tested after decarboxylation of its 3-position ester group).

\section{(+)-Ethyl 4-methyl-2-oxo-1,2,3,4-tetrahydroquinoline-3-carboxylate (3p)}

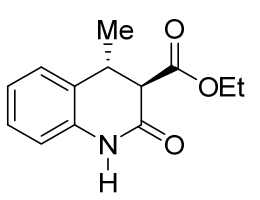

Prepared according to the general procedure from $\mathbf{2 p}(231 \mathrm{mg}, 1.0 \mathrm{mmol})$, to provide the title compound as colorless oil (eluent: petroleum ether/ethyl acetate $=10: 1$ to $2: 1), 48 \mathrm{~h}, 135 \mathrm{mg}, 58 \%$ yield $\left((R)-\mathbf{1 a}, \mathrm{S} / \mathrm{C}=250,50{ }^{\circ} \mathrm{C}\right.$ in an oil bath $), R_{\mathrm{f}}=0.3$ (petroleum ether/ethyl acetate $=2: 1), 83 \%$ ee, $8: 1$ trans $/$ cis. $[\alpha]_{\mathrm{D}}^{24}+44.8($ c 0.5 ,

$\mathrm{CHCl}_{3}$ ). ${ }^{1} \mathrm{H} \mathrm{NMR}\left(400 \mathrm{MHz}, \mathrm{CDCl}_{3}\right) \delta: 9.00(\mathrm{~s}, 1 \mathrm{H}), 7.19(\mathrm{t}, J=8.4 \mathrm{~Hz}, 2 \mathrm{H}), 7.08-6.98(\mathrm{~m}, 1 \mathrm{H}), 6.85$ (d, $J=7.6 \mathrm{~Hz}, 1 \mathrm{H}), 4.27-4.03(\mathrm{~m}, 2 \mathrm{H}), 3.66-3.45$ (m, 1H), 3.44-3.36 (m, 1H), 1.34 (d, J=6.8 Hz, 3H), $1.19(\mathrm{t}, J=7.2 \mathrm{~Hz}, 3 \mathrm{H}) ;{ }^{13} \mathrm{C} \mathrm{NMR}\left(101 \mathrm{MHz}, \mathrm{CDCl}_{3}\right) \delta: 168.9,167.3,135.6,127.9,126.9,126.8,123.7$, 115.8, 61.6, 54.8, 34.7, 19.2, 14.0; HRMS (ESI) m/z: $[\mathrm{M}+\mathrm{Na}]^{+}$calcd for $\mathrm{C}_{13} \mathrm{H}_{15} \mathrm{NO}_{3} \mathrm{Na} 256.0944$; Found 256.0948. HPLC analysis (Chiralpak AD-H column, hexane: isopropanol = 90:10; flow rate $=1 \mathrm{~mL} / \mathrm{min}$; 
UV detection at $254 \mathrm{~nm}): t_{1}=11.9 \min ($ minor $) ; t_{2}=15.3 \min ($ major $) ; t_{3}=16.4 \min ($ minor $) ; t_{4}=23.4$ $\min$ (minor).

\section{Asymmetric hydrogenation of 4-substituted 3-ethoxycarbonyl coumarins 4}<smiles>[R]c1c(C(=O)OCC)c(=O)oc2cc[R1]cc12</smiles>

4

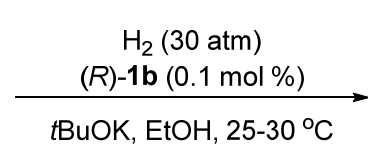

tBuOK, EtOH, $25-30^{\circ} \mathrm{C}$

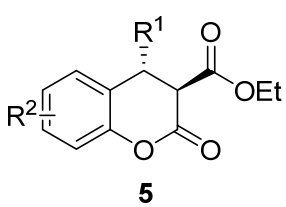

5

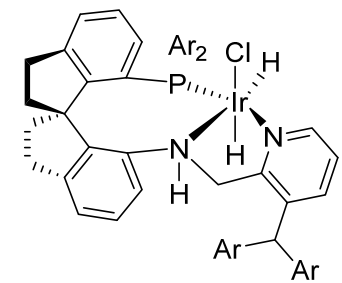

$\mathrm{Ar}=3,5-(t \mathrm{Bu})_{2} \mathrm{C}_{6} \mathrm{H}_{3}$

(R)-1b

General procedure for $\mathbf{S} / \mathbf{C}=\mathbf{1 0 0 0}$ : To a $20 \mathrm{~mL}$ hydrogenation vessel containing 3-ethoxycarbonyl coumarin $4(1.0 \mathrm{mmol})$ were added a solution of iridium catalyst $(R)-\mathbf{1 b}(1.3 \mathrm{mg}, 0.001 \mathrm{mmol})$ in EtOH $(1.0 \mathrm{~mL})$, and a solution of $t \mathrm{BuOK}(44.8 \mathrm{mg}, 0.4 \mathrm{mmol})$ in $\mathrm{EtOH}(1.0 \mathrm{~mL})$ under nitrogen atmosphere. The autoclave was purged with hydrogen by pressurizing to $5 \mathrm{~atm}$ and releasing the pressure. This procedure was repeated three times, and then pressurized to $30 \mathrm{~atm}$ of $\mathrm{H}_{2}$. The reaction mixture was stirred at room temperature $\left(25-30^{\circ} \mathrm{C}\right)$ until no obvious hydrogen pressure drop was observed. The solvent was then removed under vacuo, and the residue was redissolved with ethyl acetate $(5 \mathrm{~mL})$ and $\mathrm{NaHCO}_{3}(84 \mathrm{mg}, 1.0 \mathrm{mmol})$ was added. The resulting mixture was stirring at room temperature for $1 \mathrm{~h}$, the precipitate was filtered off, and the filtrate was concentrated in vacuo. The residue was purified by chromatographed on a silica gel with petroleum ether/ethyl acetate (10:1-5:1) to afford the desired product as a colorless oil or white solid. The enantiomeric excess of the product was determined by chiral HPLC.

\section{(+)-(3S,4R)-Ethyl-4-methyl-2-oxochromane-3-carboxylate (5a) $)^{13}$}

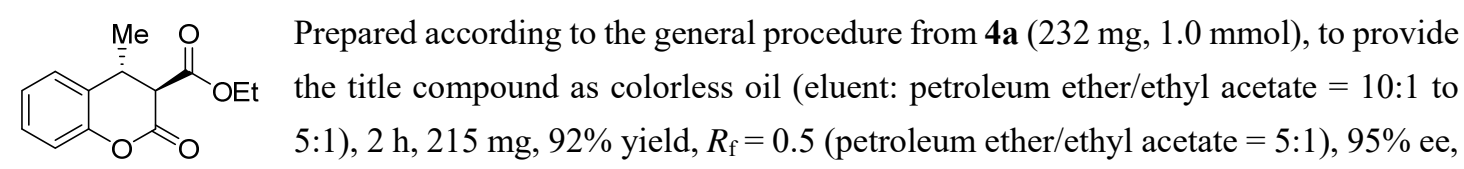
$7: 1$ (trans/cis). $[\alpha]_{\mathrm{D}}^{26}+28.4\left(\right.$ c $\left.0.5, \mathrm{CHCl}_{3}\right)$ [the absolute configuration was determined by comparing its optical rotation that reported for $(3 S, 4 R)$-isomer. lit. $^{13}[\alpha]_{\mathrm{D}}^{25}+13.8\left(c 0.5, \mathrm{CHCl}_{3}\right), 54 \%$ ee, $89: 11$ (trans/cis)]. ${ }^{1} \mathrm{H}$ NMR $\left(400 \mathrm{MHz}, \mathrm{CDCl}_{3}\right) \delta: 7.35-7.20(\mathrm{~m}, 2 \mathrm{H}), 7.14(\mathrm{td}, J=7.6,1.2 \mathrm{~Hz}, 1 \mathrm{H}), 7.08(\mathrm{dd}$, $J=8.0,1.2 \mathrm{~Hz}, 1 \mathrm{H}), 4.27-4.06$ (m, 2H), 3.80-3.40 (m, 2H), 1.37 (d, $J=6.8 \mathrm{~Hz}, 3 \mathrm{H}), 1.14$ (t, J=7.2 Hz, $3 \mathrm{H})$. HPLC analysis (Chiralpak AS-H column, hexane: isopropanol $=95: 5$; flow rate $=1 \mathrm{~mL} / \mathrm{min}$; UV detection at $210 \mathrm{~nm}$ ): $t_{1}=11.0 \mathrm{~min}$ (major); $t_{2}=13.8 \mathrm{~min}$ (minor); $t_{3}=14.1 \mathrm{~min}$ (major); $t_{2}=14.9 \mathrm{~min}$ (minor).

\section{$(+)-(3 S, 4 R)$-Ethyl-4-ethyl-2-oxochromane-3-carboxylate $(5 b)^{13}$}

Prepared according to the general procedure from $4 \mathbf{b}(246 \mathrm{mg}, 1.0 \mathrm{mmol})$, to provide
the title compound as colorless oil (eluent: petroleum ether/ethyl acetate $=10: 1$ to
$5: 1), 2 \mathrm{~h}, 249 \mathrm{mg}, 94 \%$ yield, $R_{\mathrm{f}}=0.7$ (petroleum ether/ethyl acetate $\left.=3: 1\right), 97 \%$ ee, $12: 1$ (trans/cis). $[\alpha]_{\mathrm{D}}^{26}+7.2\left(\right.$ c $\left.1.0, \mathrm{CHCl}_{3}\right)$ [the absolute configuration was determined by comparing its optical rotation that reported for $(3 S, 4 R)$-isomer. lit. ${ }^{13}[\alpha]_{\mathrm{D}}^{25}+19.4\left(c\right.$ 1.0, $\left.\mathrm{CHCl}_{3}\right), 90 \%$ ee, 93:7 (trans/cis)]. ${ }^{1} \mathrm{H}$ NMR (400 MHz, $\left.\mathrm{CDCl}_{3}\right) \delta: 7.34-7.24(\mathrm{~m}, 1 \mathrm{H}), 7.18(\mathrm{dd}, J=7.6,1.6 \mathrm{~Hz}, 1 \mathrm{H}), 7.15-7.02$ (m, 2H), 4.27-3.94 (m, 2H), 3.79 (d, $J=2.4 \mathrm{~Hz}, 1 \mathrm{H}), 3.28(\mathrm{ddd}, J=8.8,6.8,2.4 \mathrm{~Hz}, 1 \mathrm{H}), 1.75-1.54(\mathrm{~m}$, 
2H), $1.03(\mathrm{t}, J=7.2 \mathrm{~Hz}, 3 \mathrm{H}), 0.98(\mathrm{t}, J=7.4 \mathrm{~Hz}, 3 \mathrm{H})$. HPLC analysis (Chiralpak AS-H column, hexane: isopropanol $=95: 5$; flow rate $=1 \mathrm{~mL} / \mathrm{min}$; $\mathrm{UV}$ detection at $210 \mathrm{~nm}$ ): $t_{1}=9.5 \mathrm{~min}$ (major); $t_{2}=10.7 \mathrm{~min}$ (minor); $t_{3}=11.4 \min$ (major); $t_{2}=14.8 \min$ (minor).

\section{(+)-Ethyl 2-0xo-4-propylchromane-3-carboxylate $(5 \mathrm{c})^{14}$}

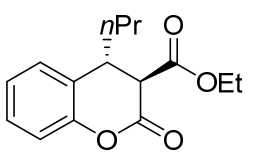

Prepared according to the general procedure from $4 \mathbf{c}(260 \mathrm{mg}, 1.0 \mathrm{mmol})$, to provide the title compound as a white solid (eluent: petroleum ether/ethyl acetate $=10: 1$ to 7:1), m.p. $55-56^{\circ} \mathrm{C} .2 \mathrm{~h}, 253 \mathrm{mg}, 95 \%$ yield, $R_{\mathrm{f}}=0.8$ (petroleum ether/ethyl acetate $=5: 1), 97 \%$ ee, 12:1 (trans/cis). $[\alpha]_{\mathrm{D}}^{23}+21.0\left(\right.$ c $\left.0.2, \mathrm{CHCl}_{3}\right) .{ }^{1} \mathrm{H}$ NMR $\left(400 \mathrm{MHz}, \mathrm{CDCl}_{3}\right) \delta: 7.36-6.95$ (m, 4H), 4.30-3.91 (m, 2H), $3.76(\mathrm{~d}, J=2.4 \mathrm{~Hz}, 1 \mathrm{H}), 3.37$ (t, $J=7.2 \mathrm{~Hz}, 1 \mathrm{H}), 1.67-1.49$ (m, 2H), $1.47-$ $1.28(\mathrm{~m}, 2 \mathrm{H}), 1.02(\mathrm{t}, J=7.0 \mathrm{~Hz}, 3 \mathrm{H}), 0.93(\mathrm{t}, J=7.2 \mathrm{~Hz}, 3 \mathrm{H})$. HPLC analysis (Chiralpak AD-H column, hexane: isopropanol $=95: 5$; flow rate $=1 \mathrm{~mL} / \mathrm{min}$; $\mathrm{UV}$ detection at $210 \mathrm{~nm}$ ): $t_{R}$ (major) $=5.8 \mathrm{~min} ; t_{R}$ $($ minor $)=6.1 \mathrm{~min}($ ee value was tested after decarboxylation of its 3-position ester group).

\section{(+)-Ethyl-4-butyl-2-oxochromane-3-carboxylate (5d)}

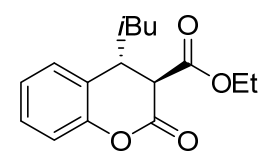

Prepared according to the general procedure from $\mathbf{4 d}(274 \mathrm{mg}, 1.0 \mathrm{mmol})$, to provide the title compound as a white solid (eluent: petroleum ether/ethyl acetate $=10: 1$ to 4:1), m.p. $67-68^{\circ} \mathrm{C} .2 \mathrm{~h}, 248 \mathrm{mg}, 90 \%$ yield, $R_{\mathrm{f}}=0.3$ (petroleum ether/ethyl acetate $=5: 1), 97 \%$ ee, $15: 1$ (trans/cis). $[\alpha]_{\mathrm{D}}^{26}+32.8\left(c 1.0, \mathrm{CHCl}_{3}\right) .{ }^{1} \mathrm{H} \mathrm{NMR}\left(400 \mathrm{MHz}, \mathrm{CDCl}_{3}\right) \delta: 7.26(\mathrm{td}, J=$ 8.0, $1.6 \mathrm{~Hz}, 1 \mathrm{H}), 7.17(\mathrm{dd}, J=7.2,1.6 \mathrm{~Hz}, 1 \mathrm{H}), 7.11(\mathrm{dd}, J=7.4,1.0 \mathrm{~Hz}, 1 \mathrm{H}), 7.07(\mathrm{~d}, J=8.0 \mathrm{~Hz}, 1 \mathrm{H})$, $4.23-3.94(\mathrm{~m}, 2 \mathrm{H}), 3.75(\mathrm{~d}, J=2.0 \mathrm{~Hz}, 1 \mathrm{H}), 3.46(\mathrm{td}, J=8.0,2.0 \mathrm{~Hz}, 1 \mathrm{H}), 1.73-1.56(\mathrm{~m}, 1 \mathrm{H}), 1.51-1.37$ (m, 2H), 1.11-0.97 (m, 6H), $0.91(\mathrm{~d}, J=6.4 \mathrm{~Hz}, 3 \mathrm{H}) ;{ }^{13} \mathrm{C} \mathrm{NMR}\left(101 \mathrm{MHz}, \mathrm{CDCl}_{3}\right) \delta: 167.1,164.5$, 150.7, 128.7, 128.4, 124.6 (2), 117.0, 62.0, 52.0, 43.5, 37.8, 24.9, 22.5, 22.3, 13.7; HRMS (ESI) m/z: $[\mathrm{M}+\mathrm{Na}]^{+}$calcd for $\mathrm{C}_{16} \mathrm{H}_{20} \mathrm{O}_{4} \mathrm{Na}$ 299.1254; Found 299.1258. HPLC analysis (Chiralpak OD-H column, hexane: isopropanol $=95: 5$; flow rate $=1 \mathrm{~mL} / \mathrm{min}$; UV detection at $210 \mathrm{~nm}$ ): $t_{R}$ (major) $=5.6 \mathrm{~min} ; t_{R}$ $($ minor $)=6.0 \mathrm{~min}$ (ee value was determined by decarboxylation to 4 -isobutylchroman-2-one) .

\section{(-)-Ethyl-4-isopropyl-2-oxochromane-3-carboxylate (5e) ${ }^{15}$}

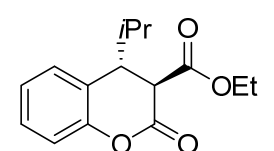

Prepared according to the general procedure from $4 \mathrm{e}(260 \mathrm{mg}, 1.0 \mathrm{mmol})$, to provide the title compound as colorless oil (eluent: petroleum ether/ethyl acetate $=10: 1$ to 5:1), $10 \mathrm{~h}, 210 \mathrm{mg}, 80 \%$ yield, $R_{\mathrm{f}}=0.7$ (petroleum ether/ethyl acetate $=3: 1$ ), $74 \%$ ee, $>20: 1$ (trans/cis). $[\alpha]_{\mathrm{D}}^{26}-9.8\left(\right.$ c 1.0, $\mathrm{CHCl}_{3}$ ). ${ }^{1} \mathrm{H} \mathrm{NMR}\left(400 \mathrm{MHz}, \mathrm{CDCl}_{3}\right) \delta: 7.20$ (ddd, $J=8.0,7.2$, $2.0 \mathrm{~Hz}, 1 \mathrm{H}), 7.08(\mathrm{dd}, J=7.4,1.8 \mathrm{~Hz}, 1 \mathrm{H}), 7.04(\mathrm{dd}, J=7.6,1.2 \mathrm{~Hz}, 1 \mathrm{H}), 7.02-6.97(\mathrm{~m}, 1 \mathrm{H}), 4.03-3.95$ (m, 1H), 3.95-3.86 (m, 1H), $3.84(\mathrm{~d}, J=2.0 \mathrm{~Hz}, 1 \mathrm{H}), 3.01(\mathrm{dd}, J=8.0,1.6 \mathrm{~Hz}, 1 \mathrm{H}), 1.77-1.64(\mathrm{~m}, 1 \mathrm{H})$, $0.95(\mathrm{~d}, J=6.8 \mathrm{~Hz}, 3 \mathrm{H}), 0.92$ (t, $J=7.2 \mathrm{~Hz}, 3 \mathrm{H}), 0.84$ (d, $J=6.4 \mathrm{~Hz}, 3 \mathrm{H})$. HPLC analysis (Chiralpak AS-H column, hexane: isopropanol $=95: 5$; flow rate $=1 \mathrm{~mL} / \mathrm{min}$; UV detection at $210 \mathrm{~nm}$ ): $t_{R}$ (major) $=7.6 \mathrm{~min} ; t_{R}(\operatorname{minor})=9.8 \mathrm{~min}$.

\section{(-)-Ethyl-4-cyclohexyl-2-oxochromane-3-carboxylate (5f)}

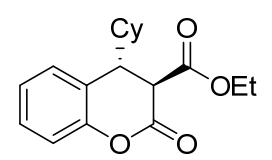

Prepared according to the general procedure from $4 \mathbf{f}(300 \mathrm{mg}, 1.0 \mathrm{mmol})$, to provide the title compound as colorless oil (eluent: petroleum ether/ethyl acetate $=10: 1$ to 5:1), $24 \mathrm{~h}, 266 \mathrm{mg}, 88 \%$ yield, $R_{\mathrm{f}}=0.8$ (petroleum ether/ethyl acetate $=3: 1$ ), 19\% ee, $>20: 1$ (trans/cis). $[\alpha]_{\mathrm{D}}^{26}-0.8\left(c 1.0, \mathrm{CHCl}_{3}\right) .{ }^{1} \mathrm{H}$ NMR $\left(400 \mathrm{MHz}, \mathrm{CDCl}_{3}\right) \delta: 7.30-7.24(\mathrm{~m}, 1 \mathrm{H}), 7.16-$ $7.05(\mathrm{~m}, 3 \mathrm{H}), 4.11-3.96(\mathrm{~m}, 2 \mathrm{H}), 3.94(\mathrm{~d}, J=1.6 \mathrm{~Hz}, 1 \mathrm{H}), 3.12(\mathrm{~d}, J=8.1 \mathrm{~Hz}, 1 \mathrm{H}), 1.89-1.56(\mathrm{~m}, 5 \mathrm{H})$, 
1.49-1.36 (m, 1H), 1.32-1.01 (m, 5H), $0.99(\mathrm{t}, J=7.2 \mathrm{~Hz}, 3 \mathrm{H}) ;{ }^{13} \mathrm{C}$ NMR (101 MHz, $\left.\mathrm{CDCl}_{3}\right) \delta: 167.6$, 165.0, 151.0, 129.8, 128.7, 124.2, 123.1, 116.8, 62.0, 53.5, 49.8, 46.0, 41.2, 30.5, 29.9, 26.0, 25.9, 13.7; HRMS (ESI) m/z: [M+Na] $]^{+}$calcd for $\mathrm{C}_{18} \mathrm{H}_{22} \mathrm{O}_{4} \mathrm{Na}$ 325.1410; Found 325.1415. HPLC analysis (Chiralpak AD-H column, hexane: isopropanol $=90: 10$; flow rate $=1 \mathrm{~mL} / \mathrm{min}$; $U V$ detection at $220 \mathrm{~nm}$ ): $t_{R}$ (major) $=5.7 \mathrm{~min} ; t_{R}($ minor $)=6.0 \mathrm{~min}$.

\section{(+)-Ethyl-4-benzyl-2-oxochromane-3-carboxylate $(5 \mathrm{~g})^{16}$}<smiles>CCOC(=O)[C@H]1C(=O)Oc2ccccc2[C@H]1Cc1ccccc1</smiles>

Prepared according to the general procedure from $\mathbf{4 g}(308 \mathrm{mg}, 1.0 \mathrm{mmol})$, to provide the title compound as a white solid (eluent: petroleum ether/ethyl acetate $=10: 1$ to 7:1), m.p. $60-61{ }^{\circ} \mathrm{C} .6 \mathrm{~h}, 256 \mathrm{mg}, 93 \%$ yield, $R_{\mathrm{f}}=0.5$ (petroleum ether/ethyl acetate $=5: 1), 96 \%$ ee, 15:1 (trans/cis). $[\alpha]_{\mathrm{D}}^{24}+88.8\left(\right.$ c 1.0, $\left.\mathrm{CHCl}_{3}\right) .{ }^{1} \mathrm{H}$ NMR $(400 \mathrm{MHz}$, $\left.\mathrm{CDCl}_{3}\right) \delta: 7.43-7.23(\mathrm{~m}, 4 \mathrm{H}), 7.17-6.71(\mathrm{~m}, 5 \mathrm{H}), 4.26-3.90(\mathrm{~m}, 2 \mathrm{H}), 3.75(\mathrm{~d}, J=1.6 \mathrm{~Hz}, 1 \mathrm{H}), 3.62$ (ddd, $J=9.2,7.0,2.2 \mathrm{~Hz}, 1 \mathrm{H}), 2.92(\mathrm{dd}, J=13.6,7.2 \mathrm{~Hz}, 1 \mathrm{H}), 2.77$ (dd, $J=13.6,9.2 \mathrm{~Hz}, 1 \mathrm{H}), 0.99$ (t, $J=7.0$ $\mathrm{Hz}, 3 \mathrm{H}$ ). HPLC analysis (Chiralpak OD-H column, hexane: isopropanol $=95: 5$; flow rate $=1 \mathrm{~mL} / \mathrm{min}$; $\mathrm{UV}$ detection at $210 \mathrm{~nm}$ ): $t_{R}$ (major) $=12.8 \mathrm{~min} ; t_{R}($ minor $)=14.52 \mathrm{~min}$ (ee value was determined by decarboxylation to 4-benzylchroman-2-one).

\section{(+)-Ethyl-2-oxo-4-phenethylchromane-3-carboxylate $(5 \mathrm{~h})$}<smiles>CCOC(=O)C1C(=O)Oc2ccccc2[C@H]1CCc1ccccc1</smiles>

Prepared according to the general procedure from $4 \mathrm{~h}(322 \mathrm{mg}, 1.0 \mathrm{mmol})$, to provide the title compound as colorless oil (eluent: petroleum ether/ethyl acetate $=10: 1$ to 5:1), 6 h, $292 \mathrm{mg}, 90 \%$ yield, $R_{\mathrm{f}}=0.6$ (petroleum ether/ethyl acetate $=5: 1$ ), 97\% ee, 15:1 (trans/cis). $[\alpha]_{\mathrm{D}}^{24}+47.8\left(\right.$ c $\left.1.0, \mathrm{CHCl}_{3}\right) .{ }^{1} \mathrm{H}$ NMR $\left(400 \mathrm{MHz}, \mathrm{CDCl}_{3}\right) \delta: 7.36-$ $7.22(\mathrm{~m}, 3 \mathrm{H}), 7.21-6.88(\mathrm{~m}, 6 \mathrm{H}), 4.23-3.95(\mathrm{~m}, 2 \mathrm{H}), 3.82(\mathrm{~d}, J=2.0 \mathrm{~Hz}, 1 \mathrm{H}), 3.42(\mathrm{t}, J=6.8 \mathrm{~Hz}, 1 \mathrm{H})$, 2.76-2.40 (m, 2H), 2.02-1.80 (m, 2H), $1.01(\mathrm{t}, J=7.2 \mathrm{~Hz}, 3 \mathrm{H}) ;{ }^{13} \mathrm{C}$ NMR $\left(101 \mathrm{MHz}, \mathrm{CDCl}_{3}\right) \delta: 167.0$, 164.4, 150.8, 140.4, 128.9, 128.7, 128.6, 128.3, 126.3, 124.7, 124.0, 117.2, 62.2, 52.0, 39.4, 35.9, 32.8, 13.8; HRMS (ESI) m/z: [M+Na] $]^{+}$calcd for $\mathrm{C}_{20} \mathrm{H}_{20} \mathrm{O}_{4} \mathrm{Na}$ 347.1254; Found 347.1258. HPLC analysis (Chiralpak AD-H column, hexane: isopropanol = 95:5; flow rate $=1 \mathrm{~mL} / \mathrm{min}$; UV detection at $210 \mathrm{~nm}$ ): $t_{R}$ (minor) $=9.4 \mathrm{~min} ; t_{R}$ (major) $=10.3 \mathrm{~min}$ (ee value was determined by decarboxylation to 4 phenethylchroman-2-one).

\section{(+)-Ethyl-4-(4-ethoxy-4-oxobutyl)-2-oxochromane-3-carboxylate (5i)}<smiles>CCOC(=O)C1C(=O)Oc2ccccc2C1C(=O)OCC</smiles>

Prepared according to the general procedure from $4 \mathbf{i}(332 \mathrm{mg}, 1.0 \mathrm{mmol})$, to provide the title compound as colorless oil (eluent: petroleum ether/ethyl acetate $=10: 1$ to $5: 1$ ), $4 \mathrm{~h}, 317 \mathrm{mg}, 95 \%$ yield, $R_{\mathrm{f}}=0.7$ (petroleum ether/ethyl acetate $=2: 1$ ), $98 \%$ ee, 13:1 (trans/cis). $[\alpha]_{\mathrm{D}}^{26}+21.8\left(\right.$ c $\left.1.0, \mathrm{CHCl}_{3}\right) .{ }^{1} \mathrm{H} \mathrm{NMR}\left(400 \mathrm{MHz}, \mathrm{CDCl}_{3}\right) \delta: 7.28(\mathrm{t}$, $J=7.5 \mathrm{~Hz}, 1 \mathrm{H}), 7.18(\mathrm{~d}, J=7.2 \mathrm{~Hz}, 1 \mathrm{H}), 7.15-6.95(\mathrm{~m}, 2 \mathrm{H}), 4.21-3.98(\mathrm{~m}, 4 \mathrm{H}), 3.77(\mathrm{~d}, J=1.9 \mathrm{~Hz}$, $1 \mathrm{H}), 3.38(\mathrm{t}, J=5.6 \mathrm{~Hz}, 1 \mathrm{H}), 2.30(\mathrm{t}, J=6.8 \mathrm{~Hz}, 2 \mathrm{H}), 1.78-1.54(\mathrm{~m}, 4 \mathrm{H}), 1.24(\mathrm{t}, J=7.2 \mathrm{~Hz}, 3 \mathrm{H}), 1.03$ $(\mathrm{t}, J=7.2 \mathrm{~Hz}, 3 \mathrm{H}) ;{ }^{13} \mathrm{C} \mathrm{NMR}\left(101 \mathrm{MHz}, \mathrm{CDCl}_{3}\right) \delta: 172.8,166.9,164.4,150.7,128.9,128.7,124.7,123.8$, $117.1,62.2,60.5,52.1,39.7,33.7,33.6,22.0,14.2,13.8 ;$ HRMS (ESI) m/z: $[\mathrm{M}+\mathrm{Na}]^{+}$calcd for $\mathrm{C}_{18} \mathrm{H}_{22} \mathrm{O}_{6} \mathrm{Na}$ 357.1309; Found 357.1315. HPLC analysis (Chiralpak OJ-H column, hexane: isopropanol $=90: 10$; flow rate $=1 \mathrm{~mL} / \mathrm{min}$; UV detection at $210 \mathrm{~nm}): t_{R}$ (major) $=21.0 \mathrm{~min} ; t_{R}($ minor $)=23.9 \mathrm{~min}$ (ee value was determined by decarboxylation to 4-(4-ethoxy-4-oxobutyl)chroman-2-one).

(+)-Ethyl-4-(2-((tert-butoxycarbonyl)(methyl)amino)ethyl)-2-oxochromane-3-carboxylate (5j) 


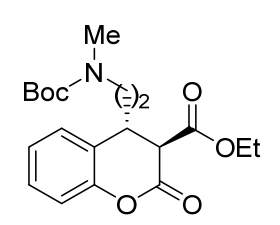

Prepared according to the general procedure from $\mathbf{4 j}$ ( $375 \mathrm{mg}, 1.0 \mathrm{mmol}$ ), to provide the title compound as colorless oil (eluent: petroleum ether/ethyl acetate $=10: 1$ to $3: 1$ ), $6 \mathrm{~h}, 350 \mathrm{mg}, 93 \%$ yield, $R_{\mathrm{f}}=0.3$ (petroleum ether/ethyl acetate $=3: 1$ ), $92 \%$ ee, $12: 1$ (trans/cis). $[\alpha]_{\mathrm{D}}^{24}+14.0\left(\right.$ c $\left.0.5, \mathrm{CHCl}_{3}\right) .{ }^{1} \mathrm{H} \mathrm{NMR}\left(400 \mathrm{MHz}, \mathrm{CDCl}_{3}\right) \delta: 7.44$ $6.92(\mathrm{~m}, 4 \mathrm{H}), 4.29-3.96(\mathrm{~m}, 2 \mathrm{H}), 3.87$ (d, $J=23.2 \mathrm{~Hz}, 1 \mathrm{H}), 3.53-3.09$ (m, 3H), 2.83 (s, 3H), 1.90-1.71 (m, 2H), $1.43(\mathrm{~d}, J=14.8 \mathrm{~Hz}, 9 \mathrm{H}), 1.03(\mathrm{t}, J=7.2 \mathrm{~Hz}, 3 \mathrm{H}) ;{ }^{13} \mathrm{C} \mathrm{NMR}(101 \mathrm{MHz}$, $\left.\mathrm{CDCl}_{3}\right) \delta: 166.7,164.2,155.5,150.7,129.0,128.4,124.8,123.9,117.1,79.8,62.1,51.8,45.6,37.2,34.0$, 31.9, 28.4, 13.8; HRMS (ESI) m/z: $[\mathrm{M}+\mathrm{Na}]^{+}$calcd for $\mathrm{C}_{20} \mathrm{H}_{27} \mathrm{NO}_{6} \mathrm{Na}$ 400.1731; Found 400.1735. HPLC analysis (Chiralpak OD-H column, hexane: isopropanol $=90: 10$; flow rate $=1 \mathrm{~mL} / \mathrm{min}$; UV detection at $210 \mathrm{~nm}): t_{R}($ major $)=7.8 \mathrm{~min} ; t_{R}$ (minor) $=8.2 \mathrm{~min}$ (ee value was determined by decarboxylation to $(2-$ ((tert-butoxycarbonyl)(methyl)amino)ethyl)chroman-2-one).

\section{(+)-(3S,4R)-Ethyl-7-methoxy-2-oxo-4-pentylchromane-3-carboxylate (5k)}<smiles>CCOC(=O)C1C(=O)Oc2cc(OC)ccc2C1COC</smiles>
Prepared according to the general procedure from $4 \mathbf{k}(318 \mathrm{mg}, 1.0 \mathrm{mmol})$, to provide the title compound as colorless oil (eluent: petroleum ether/ethyl acetate $=10: 1$ to $7: 1$ ), $3 \mathrm{~h}, 294 \mathrm{mg}, 92 \%$ yield, $R_{\mathrm{f}}=0.7$ (petroleum ether/ethyl acetate $=5: 1$ ), 98\% ee, 14:1 (trans/cis). $[\alpha]_{\mathrm{D}}^{23}+14.8$ (c 1.0, $\left.\mathrm{CHCl}_{3}\right)$ [the absolute configuration was determined by converting it to $(R)$-3-(2-hydroxy-4-methoxyphenyl)octanoic acid and comparing its optical rotation that reported for $(R)$-isomer, $[\alpha]_{\mathrm{D}}^{25}-9.9\left(c 0.58, \mathrm{CHCl}_{3}\right) ;$ it. ${ }^{17}[\alpha]_{\mathrm{D}}^{27}+6.1(c$ 1.07, $\left.\mathrm{CHCl}_{3}\right), 98 \%$ ee for (S)-acid]. ${ }^{1} \mathrm{H} \mathrm{NMR}\left(400 \mathrm{MHz}, \mathrm{CDCl}_{3}\right) \delta: 7.05(\mathrm{~d}, J=8.0 \mathrm{~Hz}, 1 \mathrm{H}), 6.66(\mathrm{dd}, J$ $=8.4,2.0 \mathrm{~Hz}, 1 \mathrm{H}), 6.63(\mathrm{~d}, J=2.0 \mathrm{~Hz}, 1 \mathrm{H}), 4.15-3.99(\mathrm{~m}, 2 \mathrm{H}), 3.79(\mathrm{~s}, 3 \mathrm{H}), 3.74(\mathrm{~d}, J=2.0 \mathrm{~Hz}, 1 \mathrm{H})$, $3.30(\mathrm{t}, J=7.2 \mathrm{~Hz}, 1 \mathrm{H}), 1.64-1.46(\mathrm{~m}, 2 \mathrm{H}), 1.38-1.22(\mathrm{~m}, 6 \mathrm{H}), 1.06(\mathrm{t}, J=7.2 \mathrm{~Hz}, 3 \mathrm{H}), 0.86(\mathrm{t}, J=6.4$ $\mathrm{Hz}, 3 \mathrm{H}) ;{ }^{13} \mathrm{C} \mathrm{NMR}\left(101 \mathrm{MHz}, \mathrm{CDCl}_{3}\right) \delta: 167.2,164.6,159.9,151.5,129.1,116.1,110.6,102.5,62.0$, 55.5, 52.3, 39.3, 34.6, 31.4, 26.2, 22.4, 13.9, 13.8; HRMS (ESI) m/z: $[\mathrm{M}+\mathrm{Na}]^{+}$calcd for $\mathrm{C}_{18} \mathrm{H}_{24} \mathrm{O}_{5} \mathrm{Na}$ 343.1516; Found 343.1520. HPLC analysis (Chiralpak OD-H column, hexane: isopropanol = 95:5; flow rate $=1 \mathrm{~mL} / \mathrm{min}$; $\mathrm{UV}$ detection at $210 \mathrm{~nm}$ ): $t_{R}$ (minor) $=6.7 \mathrm{~min} ; t_{R}$ (major) $=7.8 \mathrm{~min}$ (ee value was determined by decarboxylation to 7-methoxy-4-pentylchroman-2-one).

\section{(+)-Ethyl-4-cyclopropyl-2-oxochromane-3-carboxylate (5l)}<smiles>CCOC(=O)[C@H]1C(=O)Oc2ccccc2[C@H]1C1CC1</smiles>

Prepared according to the general procedure from $4 \mathrm{l}(258 \mathrm{mg}, 1.0 \mathrm{mmol})$, to provide the title compound as colorless oil (eluent: petroleum ether/ethyl acetate $=10: 1$ to $5: 1), 6 \mathrm{~h}, 226 \mathrm{mg}, 87 \%$ yield $(\mathrm{S} / \mathrm{C}=500), R_{\mathrm{f}}=0.4$ (petroleum ether/ethyl acetate $=$ 5:1), 96\% ee, 10:1 (trans/cis). $[\alpha]_{\mathrm{D}}^{24}+79.2$ (c 1.0, $\left.\mathrm{CHCl}_{3}\right) .{ }^{1} \mathrm{H}$ NMR (400 MHz, $\left.\mathrm{CDCl}_{3}\right) \delta: 7.29(\mathrm{t}, J=7.8 \mathrm{~Hz}, 2 \mathrm{H}), 7.20-7.01(\mathrm{~m}, 2 \mathrm{H}), 4.20-4.02(\mathrm{~m}, 2 \mathrm{H}), 3.83(\mathrm{~d}, J=4.8 \mathrm{~Hz}, 1 \mathrm{H}), 2.85$ (dd, $J=8.8,4.8 \mathrm{~Hz}, 1 \mathrm{H}), 1.10(\mathrm{t}, J=7.0 \mathrm{~Hz}, 3 \mathrm{H}), 1.00-0.75(\mathrm{~m}, 1 \mathrm{H}), 0.74-0.54(\mathrm{~m}, 2 \mathrm{H}), 0.41-0.24(\mathrm{~m}$, $2 \mathrm{H}) ;{ }^{13} \mathrm{C} \mathrm{NMR}\left(101 \mathrm{MHz}, \mathrm{CDCl}_{3}\right) \delta: 167.2,164.8,150.8,129.0,128.3,124.8,123.3,116.9,62.1,52.9$, 43.3, 15.0, 13.8, 4.0, 3.4; HRMS (ESI) m/z: $[\mathrm{M}+\mathrm{Na}]^{+}$calcd for $\mathrm{C}_{15} \mathrm{H}_{16} \mathrm{O}_{4} \mathrm{Na} 283.0941$; Found 283.0945. HPLC analysis (Chiralpak OD-H column, hexane: isopropanol $=95: 5$; flow rate $=1 \mathrm{~mL} / \mathrm{min}$; UV detection at $210 \mathrm{~nm}$ ): $t_{R}$ (major) $=11.0 \mathrm{~min} ; t_{R}$ (minor) $=12.6 \mathrm{~min}$ (ee value was determined by decarboxylation to 4-cyclopropylchroman-2-one).

\section{(+)-Ethyl-4-cyclopropyl-7-methoxy-2-oxochromane-3-carboxylate (5m)}


<smiles>CCOC(=O)C1C(=O)Oc2cc(OC)ccc2[C@H]1C1CC1</smiles>

Prepared according to the general procedure from $4 \mathrm{~m}$ (288 $\mathrm{mg}, 1.0 \mathrm{mmol})$, to provide the title compound as colorless oil (eluent: petroleum ether/ethyl acetate $=10: 1$ to $5: 1), 6 \mathrm{~h}, 264 \mathrm{mg}, 91 \%$ yield $(\mathrm{S} / \mathrm{C}=500), R_{\mathrm{f}}=0.3$ (petroleum ether/ethyl acetate $=5: 1), 96 \%$ ee, 11:1 (trans/cis). $[\alpha]_{\mathrm{D}}^{25}+73.2\left(\right.$ c 1.0, $\left.\mathrm{CHCl}_{3}\right)$. $3.74(\mathrm{~m}, 4 \mathrm{H}), 2.83(\mathrm{dd}, J=8.4,4.4 \mathrm{~Hz}, 1 \mathrm{H}), 1.13(\mathrm{t}, J=7.2 \mathrm{~Hz}, 3 \mathrm{H}), 0.95-0.73(\mathrm{~m}, 1 \mathrm{H}), 0.68-0.45(\mathrm{~m}$, 2H), 0.36-0.22 (m, 3H); $\left.{ }^{13} \mathrm{C} \mathrm{NMR} \mathrm{(101} \mathrm{MHz,} \mathrm{CDCl}_{3}\right) \delta: 167.3,164.8,160.1,151.6,128.9,114.9,110.8$, 102.3, 62.1, 55.5, 53.2, 42.6, 15.2, 13.9, 3.8, 3.1; HRMS (ESI) m/z: $[\mathrm{M}+\mathrm{Na}]^{+}$calcd for $\mathrm{C}_{16} \mathrm{H}_{18} \mathrm{O}_{5} \mathrm{Na}$ 313.1046; Found 313.1050. HPLC analysis (Chiralpak OD-H column, hexane: isopropanol = 90:10; flow rate $=1 \mathrm{~mL} / \mathrm{min}$; UV detection at $220 \mathrm{~nm}$ ): $t_{R}$ (major) $=5.3 \mathrm{~min} ; t_{R}($ minor $)=5.9$ min (ee value was determined by decarboxylation to 4-cyclopropyl-7-methoxychroman-2-one).

\section{(+)-Ethyl-4-cyclopropyl-6-methoxy-2-oxochromane-3-carboxylate (5n)}<smiles>CCOC(=O)[C@H]1C(=O)Oc2ccc(OC)cc2[C@H]1C1CC1</smiles>

Prepared according to the general procedure from $4 \mathrm{n}(288 \mathrm{mg}, 1.0 \mathrm{mmol})$, to provide the title compound as colorless oil (eluent: petroleum ether/ethyl acetate $=10: 1$ to $5: 1), 6 \mathrm{~h}, 264 \mathrm{mg}, 91 \%$ yield $(\mathrm{S} / \mathrm{C}=500), R_{\mathrm{f}}=0.4$ (petroleum ether/ethyl acetate $=5: 1), 97 \%$ ee, $11: 1$ (trans/cis). $[\alpha]_{\mathrm{D}}^{23}+88.4\left(\right.$ c $\left.0.5, \mathrm{CHCl}_{3}\right)$. ${ }^{1} \mathrm{H}$ NMR (400 MHz, $\left.\mathrm{CDCl}_{3}\right) \delta: 7.01(\mathrm{~d}, J=8.4 \mathrm{~Hz}, 1 \mathrm{H}), 6.85-6.78(\mathrm{~m}, 2 \mathrm{H}), 4.20-4.07(\mathrm{~m}, 2 \mathrm{H}), 3.80(\mathrm{~s}$, $3 \mathrm{H}), 2.79(\mathrm{dd}, J=8.8,5.2 \mathrm{~Hz}, 1 \mathrm{H}), 1.13(\mathrm{t}, J=7.2 \mathrm{~Hz}, 3 \mathrm{H}), 0.97-0.86(\mathrm{~m}, 1 \mathrm{H}), 0.68-0.55(\mathrm{~m}, 2 \mathrm{H}), 0.40-$ 0.28 (m, 2H); ${ }^{13} \mathrm{C}$ NMR (101 MHz, $\left.\mathrm{CDCl}_{3}\right) \delta: 167.2,164.9,156.4,144.6,124.4,117.6,113.7,113.5$, 62.0, 55.6, 52.9, 43.4, 15.0, 13.9, 4.1, 3.4; HRMS (ESI) m/z: $[\mathrm{M}+\mathrm{H}]^{+}$calcd for $\mathrm{C}_{16} \mathrm{H}_{19} \mathrm{O}_{5}$ 291.1227; Found 291.1230. HPLC analysis (Chiralpak OD-H column, hexane: isopropanol = 95:5; flow rate $=1$ $\mathrm{mL} / \mathrm{min}$; UV detection at $210 \mathrm{~nm}$ ): $t_{R}$ (minor) $=11.2 \mathrm{~min} ; t_{R}$ (major) $=13.2 \mathrm{~min}$ (ee value was determined by decarboxylation to 4-cyclopropyl-6-methoxychroman-2-one).

\section{(-)-(3R,4R)-Ethyl-2-oxo-4-phenylchromane-3-carboxylate (5o) $)^{18}$}

Prepared according to the general procedure from $40(294 \mathrm{mg}, 1.0 \mathrm{mmol})$, to provide
the title compound as colorless oil (eluent: petroleum ether/ethyl acetate $=10: 1$ to ee, $>20: 1$ (trans/cis). $[\alpha]_{\mathrm{D}}^{25}-62.6\left(c \quad 0.86, \mathrm{CHCl}_{3}\right)$ [the absolute configuration was determined by comparing its optical rotation that reported for $(3 R, 4 R)$-isomer. lit. ${ }^{18}[\alpha]_{\mathrm{D}}^{20}-72.3\left(c 0.86, \mathrm{CHCl}_{3}\right), 90 \%$ ee, >20:1 trans/cis]. ${ }^{1} \mathrm{H}$ NMR (400 MHz, $\left.\mathrm{CDCl}_{3}\right) \delta: 7.40-7.26(\mathrm{~m}, 2 \mathrm{H}), 7.21-7.12(\mathrm{~m}, 3 \mathrm{H}), 7.10(\mathrm{td}, J=$ 7.6, 1.2 Hz, 1H), 6.95 (dt, $J=7.6,1.2 \mathrm{~Hz}, 1 \mathrm{H}), 4.73(\mathrm{~d}, J=8.0 \mathrm{~Hz}, 1 \mathrm{H}), 4.19-4.01(\mathrm{~m}, 1 \mathrm{H}), 3.97$ (d, $J=$ $8.0 \mathrm{~Hz}, 1 \mathrm{H}), 1.06(\mathrm{t}, J=7.2 \mathrm{~Hz}, 3 \mathrm{H})$. HPLC analysis (Chiralpak AD-H column, hexane: isopropanol = 90:10; flow rate $=1 \mathrm{~mL} / \mathrm{min}$; $\mathrm{UV}$ detection at $220 \mathrm{~nm}): t_{R}($ major $)=9.7 \mathrm{~min} ; t_{R}($ minor $)=12.3 \mathrm{~min}$.

Asymmetric hydrogenation of $4 \mathbf{a}$ at $\mathbf{S} / \mathbf{C}=\mathbf{3 0 0 0 0}$ : To a $60 \mathrm{~mL}$ hydrogenation vessel containing ethyl 2-oxo-2H-chromene-3-carboxylate $4 \mathbf{a}(6.96 \mathrm{~g}, 30.0 \mathrm{mmol})$ was added a solution of iridium catalyst $(R)$ $\mathbf{1 b}(1.36 \mathrm{mg}, 0.001 \mathrm{mmol})$ in $\mathrm{EtOH}(1.0 \mathrm{~mL})$, a solution of $t \mathrm{BuOK}(672 \mathrm{mg}, 6 \mathrm{mmol})$ in $\mathrm{EtOH}(40.0 \mathrm{~mL})$, and toluene $(5 \mathrm{~mL})$ as co-solvent under nitrogen atmosphere. The autoclave was purged with hydrogen by pressurizing to $5 \mathrm{~atm}$ and releasing the pressure. This procedure was repeated three times and then pressurized to $60 \mathrm{~atm}$ of $\mathrm{H}_{2}$. The reaction mixture was stirred at room temperature $\left(25-30{ }^{\circ} \mathrm{C}\right)$ for $65 \mathrm{~h}$. After releasing the hydrogen pressure, the solvent was then removed under vacuo. The residue was then redissolved with ethyl acetate $(50 \mathrm{~mL})$ and $\mathrm{NaHCO}_{3}(840 \mathrm{mg}, 10.0 \mathrm{mmol})$ was added. The resulting 
mixture was then stirred at room temperature for $2 \mathrm{~h}$, the precipitate was filtered off, and the filtrate was concentrated in vacuo. The residue was purified by chromatographed on a silica gel with petroleum ether/ethyl acetate (5:1) to afford $(3 S, 4 R)-5 a$ in $92 \%$ yield $(6.46 \mathrm{~g},>99 \%$ conversion) with $95 \%$ ee (trans/cis $=7: 1$ ).

\section{(C) Conversion of the Hydrogenated Products}

\section{Enantioselective synthesis of the key intermediate (S)-7 for R-106578 ${ }^{17}$}

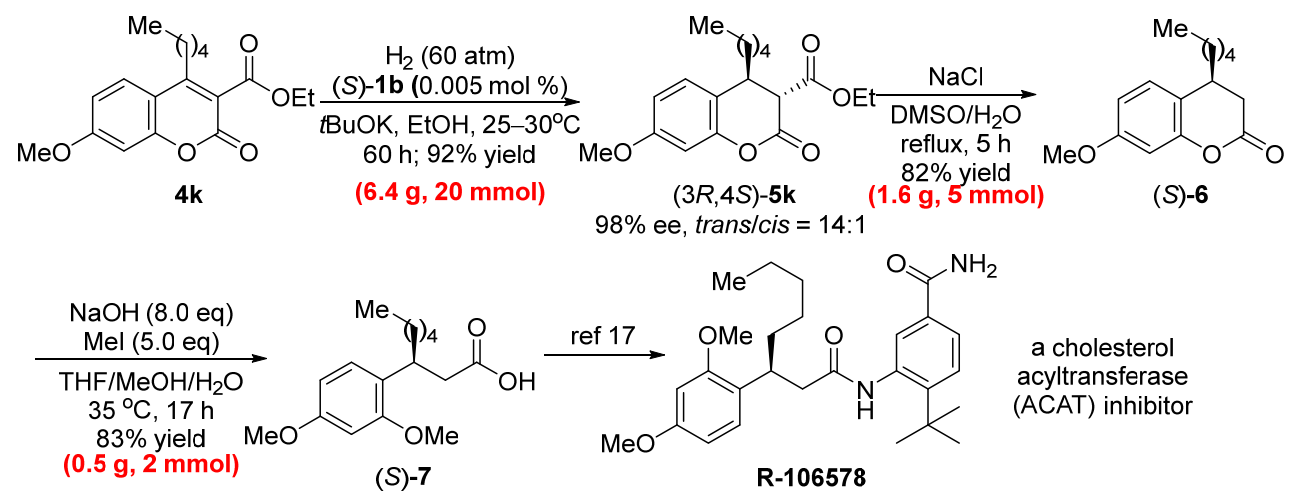

\section{Synthesis of $(3 R, 4 S)-5 \mathrm{k}$ at a low catalyst loading $(\mathrm{S} / \mathrm{C}=\mathbf{2 0 0 0 0})$}

To a $60 \mathrm{~mL}$ hydrogenation vessel containing ethyl 2-oxo- $2 H$-chromene-3-carboxylate $4 \mathbf{k}$ (6.36 g, 20.0 mmol) was added a solution of iridium catalyst $(S)-\mathbf{1 b}(1.36 \mathrm{mg}, 0.001 \mathrm{mmol})$ in EtOH $(1.0 \mathrm{~mL})$ and a solution of $t \mathrm{BuOK}(448 \mathrm{mg}, 4.0 \mathrm{mmol})$ in $\mathrm{EtOH}(20.0 \mathrm{~mL})$ under nitrogen atmosphere. The autoclave was purged with hydrogen by pressurizing to $5 \mathrm{~atm}$ and releasing the pressure. This procedure was repeated three times and then pressurized to $60 \mathrm{~atm}$ of $\mathrm{H}_{2}$. The reaction mixture was stirred at room temperature $\left(25-30{ }^{\circ} \mathrm{C}\right)$ for $60 \mathrm{~h}$. After releasing the hydrogen pressure, the solvent was then removed under vacuo. The residue was then redissolved with ethyl acetate $(50 \mathrm{~mL})$ and $\mathrm{NaHCO}_{3}(840 \mathrm{mg}, 10.0$ mmol) was added. The resulting mixture was then stirred at room temperature for $2 \mathrm{~h}$, the precipitate was filtered off, and the filtrate was concentrated in vacuo. The residue was purified by chromatographed on a silica gel with petroleum ether/ethyl acetate $(5: 1)$ to afford $(3 R, 4 S)-\mathbf{5 k}$ as a colorless oil in $94 \%$ yield $(6.02 \mathrm{~g}, 98 \%$ conversion) with $98 \%$ ee (trans/cis $=14: 1) .[\alpha]_{\mathrm{D}}^{25}-15.6\left(c\right.$ 1.0, $\left.\mathrm{CHCl}_{3}\right)$ (the absolute configuration was determined by converting it to $(S)$-3-(2-hydroxy-4-methoxyphenyl)octanoic acid and comparing its optical rotation that reported for $(S)$-isomer, $[\alpha]_{\mathrm{D}}^{25}+6.4\left(c 1.07, \mathrm{CHCl}_{3}\right)\left(\mathrm{lit}^{16}[\alpha]_{\mathrm{D}}^{27}+6.1(c\right.$ $\left.1.07, \mathrm{CHCl}_{3}\right), 98 \%$ ee for (S)- isomer).

\section{Synthesis of $(S)-6$}

A mixture of $(3 R, 4 S)$-ethyl-7-methoxy-2-oxo-4-pentylchromane-3-carboxylate ((3R,4S)-5k) $(1.60 \mathrm{~g}, 5.0$ mmol), $\mathrm{NaCl}(615 \mathrm{mg}, 10.5 \mathrm{mmol}), \mathrm{H}_{2} \mathrm{O}(90 \mu \mathrm{L}, 5.0 \mathrm{mmol})$ and DMSO (15 mL) was heated to reflux in an oil bath for $5 \mathrm{~h}$. The mixture was then cooled to room temperature, diluted with $\mathrm{H}_{2} \mathrm{O}(30 \mathrm{~mL})$, and extracted with ethyl acetate $(3 \times 20 \mathrm{~mL})$. The combined extracts were washed with brine $(20 \mathrm{~mL})$, dried over $\mathrm{MgSO}_{4}$, and concentrated in vacuo. The residue was purified by flash chromatography on silica gel with petroleum ether/ethyl acetate $(10: 1)$ as an eluent to give (S)-7-methoxy-4-pentylchroman-2-one ((S)-6) as a colorless oil. $1.02 \mathrm{~g}, 82 \%$ yield. $[\alpha]_{\mathrm{D}}^{27}-46.6$ (c 1.0, $\left.\mathrm{CHCl}_{3}\right) .{ }^{1} \mathrm{H} \mathrm{NMR}\left(400 \mathrm{MHz}, \mathrm{CDCl}_{3}\right) \delta$ : $7.07(\mathrm{~d}, J=8.4 \mathrm{~Hz}, 1 \mathrm{H}), 6.66(\mathrm{dd}, J=8.4,2.5 \mathrm{~Hz}, 1 \mathrm{H}), 6.59(\mathrm{~d}, J=2.5 \mathrm{~Hz}, 1 \mathrm{H}), 3.78(\mathrm{~s}, 3 \mathrm{H}), 2.97-2.87$ (m, 1H), $2.80(\mathrm{dd}, J=16.0,5.6 \mathrm{~Hz}, 1 \mathrm{H}), 2.70(\mathrm{dd}, J=16.0,4.0 \mathrm{~Hz}, 1 \mathrm{H}), 1.62-1.45(\mathrm{~m}, 2 \mathrm{H}), 1.42-1.22$ 


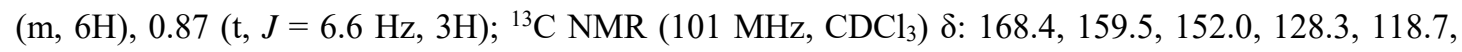
110.1, 102.5, 55.4, 35.0, 34.8, 34.4, 31.6, 26.3, 22.5, 14.0; HRMS (ESI) m/z: $[\mathrm{M}+\mathrm{Na}]^{+}$calcd for $\mathrm{C}_{15} \mathrm{H}_{20} \mathrm{O}_{3} \mathrm{Na} 271.1305$; Found 271.1310.

\section{Synthesis of $(S)-7^{17}$}

A mixture of (S)-7-methoxy-4-pentylchroman-2-one ((S)-6) (496 mg, $2.0 \mathrm{mmol}), \mathrm{NaOH}$ (645 mg, 16.0 mmol), THF (24 mL), MeOH (6 mL), and $\mathrm{H}_{2} \mathrm{O}(6 \mathrm{~mL})$ was stirred at $35^{\circ} \mathrm{C}$ in an oil bath for $17 \mathrm{~h}$. The reaction mixture was then acidified to $\mathrm{pH}<2$ with an aqueous $\mathrm{HCl}$ solution $(1 \mathrm{~N}, 30.0 \mathrm{~mL}, 30 \mathrm{mmol})$ at $0{ }^{\circ} \mathrm{C}$, and extracted with ethyl acetate $(3 \times 20 \mathrm{~mL})$. The combined extracts were washed with brine $(20$ $\mathrm{mL}$ ), dried over $\mathrm{MgSO}_{4}$, and concentration in vacuo. The residue was purified by flash chromatography on silica gel with petroleum ether/ethyl acetate (10:1 to 5:1) as an eluent to give (S)-3-(2,4dimethoxyphenyl)octanoic acid $((S)-7)$ as a colorless oil. $465 \mathrm{mg}, 83 \%$ yield $\left([\alpha]_{\mathrm{D}}^{25}+6.4\left(c 1.07, \mathrm{CHCl}_{3}\right)\right.$ (lit. ${ }^{17}[\alpha]_{\mathrm{D}}^{27}+6.1$ (c 1.07, $\left.\mathrm{CHCl}_{3}\right), 98 \%$ ee for (S)-isomer). ${ }^{1} \mathrm{H}$ NMR $\left(400 \mathrm{MHz}, \mathrm{CDCl}_{3}\right) \delta: 10.99(\mathrm{~s}, 1 \mathrm{H})$, 7.09-6.93 (m, 1H), 6.53-6.35 (m, 2H), 3.79 (s, 3H), 3.77 (s, 3H), 3.45-3.33 (m, 1H), 2.69-2.54 (m, 2H), $1.72-1.53(\mathrm{~m}, 2 \mathrm{H}), 1.30-1.12(\mathrm{~m}, 6 \mathrm{H}), 0.83(\mathrm{t}, J=6.4 \mathrm{~Hz}, 3 \mathrm{H})$.

\section{Enantioselective synthesis of MPR3160 ${ }^{19}$}

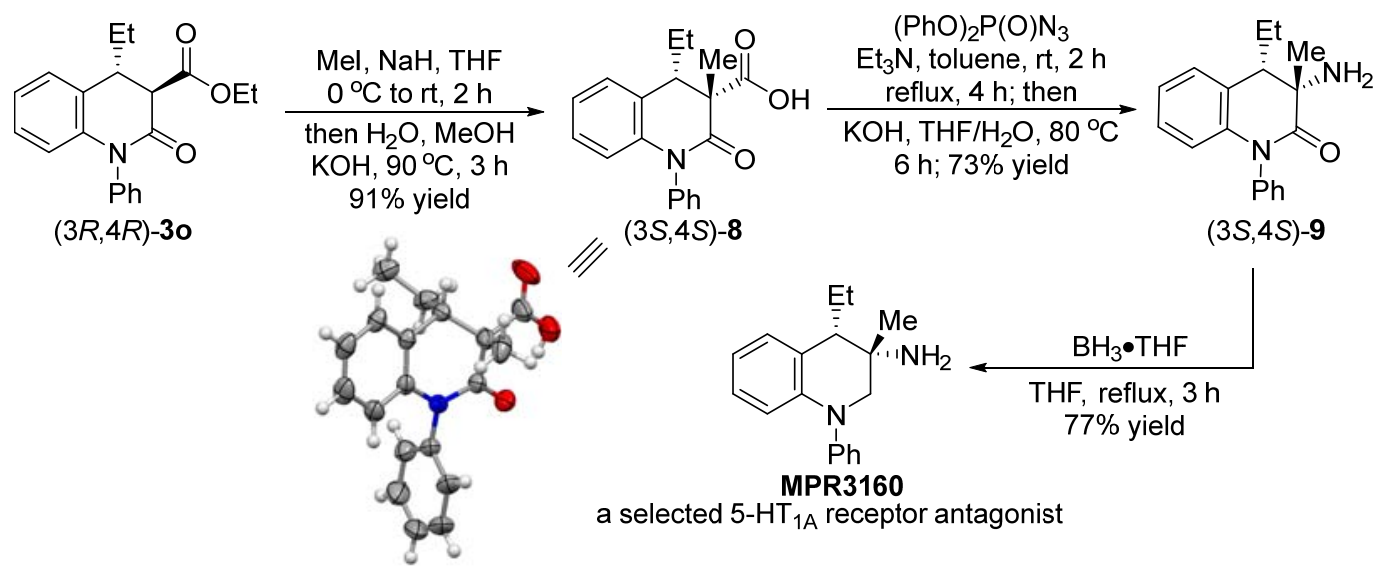

\section{Synthesis of $(3 S, 4 S)-8$}

To a stirring solution of (3R,4R)-30 (920 mg, $2.8 \mathrm{mmol})$ in THF $(20 \mathrm{~mL}), 60 \% \mathrm{NaH}(170 \mathrm{mg}, 4.3 \mathrm{mmol})$ was added slowly and stirred at $0{ }^{\circ} \mathrm{C}$ for $30 \mathrm{~min}$. MeI $(266 \mu \mathrm{L}, 4.27 \mathrm{mmol})$ was then added at $0{ }^{\circ} \mathrm{C}$ and stirred at room temperature for $2 \mathrm{~h}$. The mixture was then quenched with $\mathrm{H}_{2} \mathrm{O}(5 \mathrm{~mL})$, and a solution of $\mathrm{KOH}(797 \mathrm{mg}, 14.2 \mathrm{mmol})$ in $\mathrm{MeOH}(10 \mathrm{~mL})$ was added, and the mixture was heated to $90{ }^{\circ} \mathrm{C}$ in an oil bath for $6 \mathrm{~h}$. After cooling to room temperature, the mixture was diluted with water $(10 \mathrm{~mL})$, and acidified with $2 \mathrm{~N} \mathrm{HCl}(20 \mathrm{~mL})$ to $\mathrm{pH}<4$, and extracted with ethyl acetate $(3 \times 20 \mathrm{~mL})$. The combined extracts were washed with brine $(20 \mathrm{~mL})$, dried over $\mathrm{MgSO}_{4}$, and concentrated in vacuo. The residue was purified by chromatography on silica gel column with petroleum ether/ethyl acetate (5:1 to 1:1) as eluent to afford the corresponding acid $(3 S, 4 S)-8$ as a white solid. m.p. $118-119{ }^{\circ} \mathrm{C}, 0.80 \mathrm{~g}, 91 \%$ yield. $R_{\mathrm{f}}=0.2$ (petroleum ether/ethyl acetate $=2: 1),>20: 1$ trans/cis. $[\alpha]_{\mathrm{D}}^{27}+36.0\left(\right.$ c 1.0, $\left.\mathrm{CHCl}_{3}\right) .{ }^{1} \mathrm{H} \mathrm{NMR}(400 \mathrm{MHz}$, $\left.\mathrm{CDCl}_{3}\right) \delta: 13.95(\mathrm{~s}, 1 \mathrm{H}), 7.64-7.44(\mathrm{~m}, 3 \mathrm{H}), 7.32-7.08(\mathrm{~m}, 5 \mathrm{H}), 6.49-6.38(\mathrm{~m}, 1 \mathrm{H}), 3.21(\mathrm{dd}, J=11.2$, $3.2 \mathrm{~Hz}, 1 \mathrm{H}), 1.87-1.73(\mathrm{~m}, 1 \mathrm{H}), 1.53(\mathrm{~s}, 3 \mathrm{H}), 1.52-1.39(\mathrm{~m}, 1 \mathrm{H}), 0.94(\mathrm{t}, J=7.0 \mathrm{~Hz}, 3 \mathrm{H}) ;{ }^{13} \mathrm{C} \mathrm{NMR}(101$ $\left.\mathrm{MHz}, \mathrm{CDCl}_{3}\right) \delta: 175.1,173.5,137.9,136.9,130.3,130.1,129.2,128.2,127.9,126.6,124.8,117.8,49.8$, 47.3, 25.0, 22.8, 11.9. HRMS (ESI) m/z: $[\mathrm{M}+\mathrm{Na}]^{+}$calcd for $\mathrm{C}_{19} \mathrm{H}_{19} \mathrm{NO}_{3} \mathrm{Na} 332.1257$; Found 332.1261. 
The product $\mathbf{8}$ was resolved in a small amount of ethyl acetate and layered with hexane to yield $\mathbf{8}$ as colorless crystals suitable for X-ray diffraction analysis. The intensity data were collected on an a Rigaku 002 Saturn 944 diffractometer using graphite-monochromated $\mathrm{Cu} \mathrm{K \alpha}(\lambda=1.54184 \AA)$ radiation. The absolute configuration of $\mathbf{8}$ was determined as $(3 S, 4 S)$. The crystal structure (ORTEP representation, $50 \%$ thermal probability ellipsoids) and the data were outlined below.<smiles>C#CC1c2ccccc2N([13CH3])C(=O)[C@]1(CC)C(=O)O</smiles>

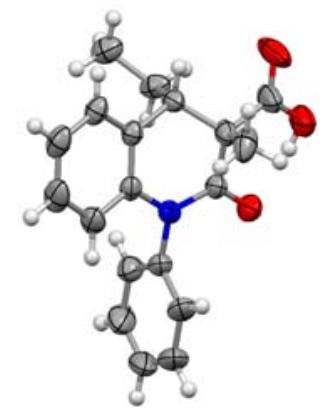

Table S1. Crystal data and structure refinement for 8 (CCDC 2060089).

\begin{tabular}{|c|c|}
\hline Identification code & p20201115c \\
\hline Empirical formula & $\mathrm{C}_{19} \mathrm{H}_{19} \mathrm{NO}_{3}$ \\
\hline Formula weight & 309.35 \\
\hline Temperature/K & 294.15 \\
\hline Crystal system & monoclinic \\
\hline Space group & $\mathrm{P} 2_{1}$ \\
\hline $\mathrm{a} / \AA$ & $11.15519(5)$ \\
\hline $\mathrm{b} / \AA$ & $9.83926(4)$ \\
\hline $\mathrm{c} / \AA$ & $15.50579(7)$ \\
\hline$\alpha /^{\circ}$ & 90 \\
\hline$\beta /^{\circ}$ & $96.2996(4)$ \\
\hline$\gamma /^{\circ}$ & 90 \\
\hline Volume $/ \AA^{3}$ & 1691.620(13) \\
\hline $\mathrm{Z}$ & 4 \\
\hline$\rho_{\text {calc }} \mathrm{g} / \mathrm{cm}^{3}$ & 1.215 \\
\hline$\mu / \mathrm{mm}^{-1}$ & 0.663 \\
\hline $\mathrm{F}(000)$ & 656.0 \\
\hline Crystal size $/ \mathrm{mm}^{3}$ & $0.17 \times 0.15 \times 0.14$ \\
\hline Radiation & $\operatorname{CuK} \alpha(\lambda=1.54184)$ \\
\hline $2 \Theta$ range for data collection $/{ }^{\circ}$ & 7.974 to 158.11 \\
\hline Index ranges & $-14 \leq \mathrm{h} \leq 13,-12 \leq \mathrm{k} \leq 12,-19 \leq \mathrm{l} \leq 19$ \\
\hline Reflections collected & 32699 \\
\hline Independent reflections & $7023\left[\mathrm{R}_{\text {int }}=0.0208, R_{\text {sigma }}=0.0153\right]$ \\
\hline Data/restraints/parameters & $7023 / 1 / 422$ \\
\hline Goodness-of-fit on $\mathrm{F}^{2}$ & 1.063 \\
\hline Final $R$ indices $[I \geq 2 \sigma(I)]$ & $\mathrm{R}_{1}=0.0375, \mathrm{wR}_{2}=0.1090$ \\
\hline Final $\mathrm{R}$ indices (all data) & $\mathrm{R}_{1}=0.0381, \mathrm{wR}_{2}=0.1097$ \\
\hline Largest diff. peak/hole / e $\AA^{-3}$ & $0.17 /-0.11$ \\
\hline Flack parameter & $0.04(5)$ \\
\hline
\end{tabular}




\section{Synthesis of $(3 S, 4 S)-9$}

To a solution of carboxylic acid $8(309 \mathrm{mg}, 1.0 \mathrm{mmol})$ in toluene $(4 \mathrm{~mL})$ was added $(\mathrm{PhO})_{2} \mathrm{P}(\mathrm{O}) \mathrm{N}_{3}(320$ $\mu \mathrm{L}, 1.5 \mathrm{mmol})$ and $\mathrm{Et}_{3} \mathrm{~N}(350 \mu \mathrm{L}, 2.5 \mathrm{mmol})$ at room temperature. The reaction mixture was stirred at room temperature for $2.0 \mathrm{~h}$ and heated to reflux in an oil bath for $6.0 \mathrm{~h}$, and concentrated in vacuo. Then, the residue was redissolved with a solution of $\mathrm{KOH}(560 \mathrm{mg}, 10.0 \mathrm{mmol})$ in THF $(4 \mathrm{~mL})$ and $\mathrm{H}_{2} \mathrm{O}(2$ $\mathrm{mL}$ ), the resulting mixture was heated to $80^{\circ} \mathrm{C}$ in an oil bath for $6 \mathrm{~h}$. The mixture was diluted with water $(10 \mathrm{~mL})$, and extracted with ethyl acetate $(3 \times 20 \mathrm{~mL})$. The combined extracts were then washed with brine $(20 \mathrm{~mL})$, dried over $\mathrm{MgSO}_{4}$, and concentrated in vacuo. The residue was then purified by chromatography on silica gel column with petroleum ether/ethyl acetate (2:1) to $\mathrm{CH}_{2} \mathrm{Cl}_{2} / \mathrm{MeOH}$ (10:1) as eluent to yield (3S,4S)-9 as a colorless oil. $183 \mathrm{mg}, 73 \%$ yield. $R_{\mathrm{f}}=0.5\left(\mathrm{CH}_{2} \mathrm{Cl}_{2} / \mathrm{MeOH}=5: 1\right),>20: 1$ trans/cis. $[\alpha]_{\mathrm{D}}^{22}-18.2\left(c 1.0, \mathrm{CHCl}_{3}\right) .{ }^{1} \mathrm{H}$ NMR $\left(400 \mathrm{MHz}, \mathrm{CDCl}_{3}\right) \delta: 7.50(\mathrm{t}, J=7.6 \mathrm{~Hz}, 2 \mathrm{H}), 7.41(\mathrm{t}, J$ $=7.4 \mathrm{~Hz}, 1 \mathrm{H}), 7.26-7.10(\mathrm{~m}, 3 \mathrm{H}), 7.09-6.90(\mathrm{~m}, 2 \mathrm{H}), 6.35(\mathrm{~d}, J=7.6 \mathrm{~Hz}, 1 \mathrm{H}), 2.65(\mathrm{dd}, J=10.6,3.4$ $\mathrm{Hz}, 1 \mathrm{H}), 2.03-1.84(\mathrm{~m}, 3 \mathrm{H}), 1.50-1.36(\mathrm{~m}, 1 \mathrm{H}), 1.31(\mathrm{~s}, 3 \mathrm{H}), 0.91(\mathrm{t}, J=7.2 \mathrm{~Hz}, 3 \mathrm{H}) ;{ }^{13} \mathrm{C}$ NMR $(101$ $\left.\mathrm{MHz}, \mathrm{CDCl}_{3}\right)$ ס: 175.8, 139.7, 138.70, 129.8, 129.8, 128.7, 128.2, 128.2, 127.2, 122.8, 117.0, 56.4, 50.9, 26.0, 22.3, 11.7. HRMS (ESI) m/z: [M+H] calcd for $\mathrm{C}_{18} \mathrm{H}_{21} \mathrm{~N}_{2} \mathrm{O}$ 281.1648; Found 281.1651.

\section{Synthesis of MPR3160 ${ }^{19}$}

To a solution of amine $9(140 \mathrm{mg}, 0.5 \mathrm{mmol})$ in dry THF $(4 \mathrm{~mL})$ was added $\mathrm{BH}_{3} \cdot \mathrm{THF}(5 \mathrm{~mL}, 1 \mathrm{M}$ in THF, $5.0 \mathrm{mmol}$ ). The reaction mixture was then heated to reflux in an oil bath for $3 \mathrm{~h}$, neutralized with 6 $\mathrm{N} \mathrm{HCl}(10 \mathrm{~mL})$ until no hydrogen evolved from the solution. The mixture was then continued to stir at room temperature for $30 \mathrm{~min}$, and an aqueous solution of $\mathrm{NaOH}(1 \mathrm{M})$ was added to adjust the $\mathrm{pH}$ at 14 . The mixture was extracted with $\mathrm{CH}_{2} \mathrm{Cl}_{2}(3 \times 20 \mathrm{~mL})$, and the combined extracts were then washed with brine $(20 \mathrm{~mL})$, dried over $\mathrm{MgSO}_{4}$, and concentrated in vacuo. The residue was purified by chromatography on silica gel column with $\mathrm{CH}_{2} \mathrm{Cl}_{2} / \mathrm{MeOH}$ (10:1) as eluent to afford MPR3160 as a colorless oil. $102 \mathrm{mg}, 77 \%$ yield. $R_{\mathrm{f}}=0.4\left(\mathrm{CH}_{2} \mathrm{Cl}_{2} / \mathrm{MeOH}=5: 1\right),>20: 1$ cis/trans. $[\alpha]_{\mathrm{D}}^{22}+28.0(c \mathrm{c} 0.5$, $\mathrm{CHCl}_{3}$ ). ${ }^{1} \mathrm{H}$ NMR (400 MHz, $\left.\mathrm{CDCl}_{3}\right)$ 8: 7.41-7.30 (m, 2H), 7.26-7.17 (m, 2H), $7.12(\mathrm{tt}, J=7.2,1.2 \mathrm{~Hz}$, $1 \mathrm{H}), 7.03$ (dd, $J=7.6,1.6 \mathrm{~Hz}, 1 \mathrm{H}), 6.95$ (ddd, $J=8.6,7.4,1.6 \mathrm{~Hz}, 1 \mathrm{H}), 6.80-6.56(\mathrm{~m}, 2 \mathrm{H}), 3.45$ (d, $J=$ $11.2 \mathrm{~Hz}, 1 \mathrm{H}), 3.35(\mathrm{dd}, J=11.0,1.8 \mathrm{~Hz}, 1 \mathrm{H}), 2.39-2.27(\mathrm{~m}, 1 \mathrm{H}), 1.96-1.84(\mathrm{~m}, 1 \mathrm{H}), 1.54(\mathrm{~s}, 2 \mathrm{H}), 1.40$ $1.29(\mathrm{~m}, 1 \mathrm{H}), 1.17(\mathrm{~s}, 3 \mathrm{H}), 1.01(\mathrm{t}, J=7.4 \mathrm{~Hz}, 3 \mathrm{H})$.

\section{(D) Computational Study}

\section{DFT Methods.}

All DFT calculations have been carried out using the Gaussian 16 program package. ${ }^{20}$ The B3LYP ${ }^{21}$ method with def2svp basis set has been selected for geometry optimizations and calculation of Gibbs energy corrections at $298 \mathrm{~K}$. Final energies were retrieved from single-point calculations at the B3LYP/6$311+\mathrm{G}^{* *}$ level, including the GD3BJ dispersion correction scheme developed by Grimme. ${ }^{22}$ All structures have been optimized considering solvent effects using the CPCM Model for MeOH. Reaction paths were traced by the intrinsic reaction coordinate method for all transition states. All energetics reported throughout the text are in $\mathrm{kcal} / \mathrm{mol}$. Structures were generated using CYLview. ${ }^{23}$

\section{DFT Structures}

The absolute configuration of iridium catalyst is determined by crystal structure. The chiral iridiumhydride intermediates can be generated in situ from the reaction mixture, and may act as an effective 
reductant for the upper-face or down-face attack on the highly activated $\mathrm{C}=\mathrm{C}$ bond of the substrate via a six-membered cyclic transition (TS), assuming a Noyori bifunctional mechanism like ketones.

(a)

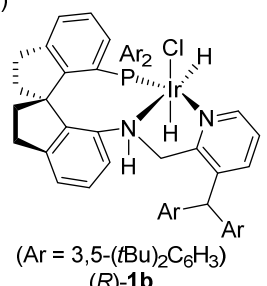

(b)

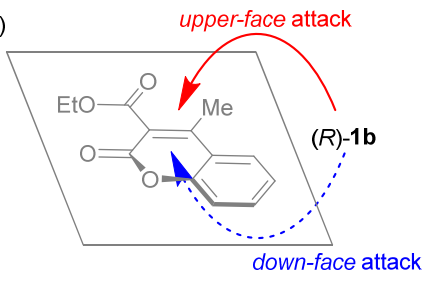

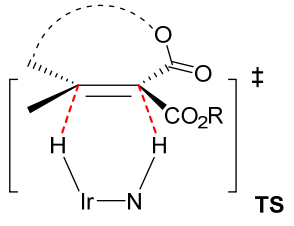

schematic representation of the transition state for $\mathrm{H}^{-} / \mathrm{H}^{+}$transfer bifunctional mechanism

The two models of transition state structures for the $\mathrm{H}^{-} / \mathrm{H}^{+}$transfer to $\mathbf{4 a}$ were simulated in Figure $\mathbf{S 1}$. In this process, the bulky group at the 3-position of pyridine ring and the rigid spiro backbone of catalyst directed the approach of the substrate to the catalyst. To minimize the steric repulsion, TS-RR were favorable for $\mathbf{4 a}$, leading to the formation of $(3 R, 4 R)-\mathbf{5} \mathbf{a}$. Since cis-product $(3 R, 4 R)-\mathbf{5} \mathbf{a}$ was prone to epimerize to thermodynamically more stable trans-isomer $(3 S, 4 R)-5 \mathbf{a}$ under basic conditions, a mixture products dominated by trans-isomer $(3 S, 4 R)-\mathbf{5 a}$ was finally observed.

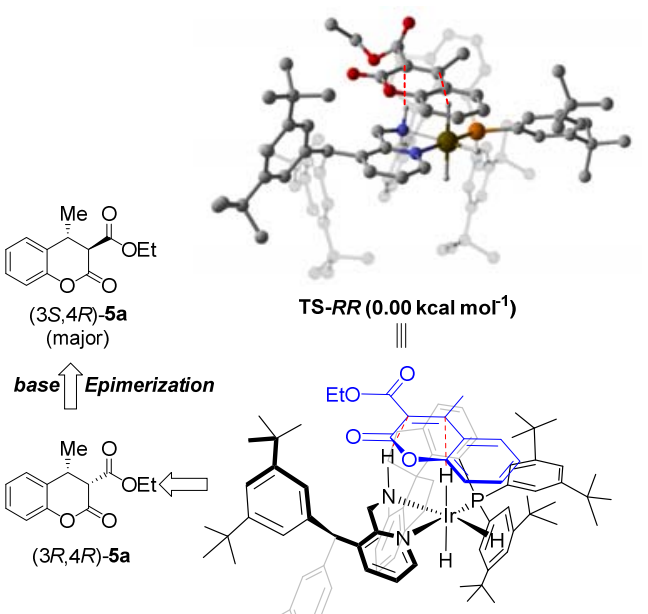

favorable

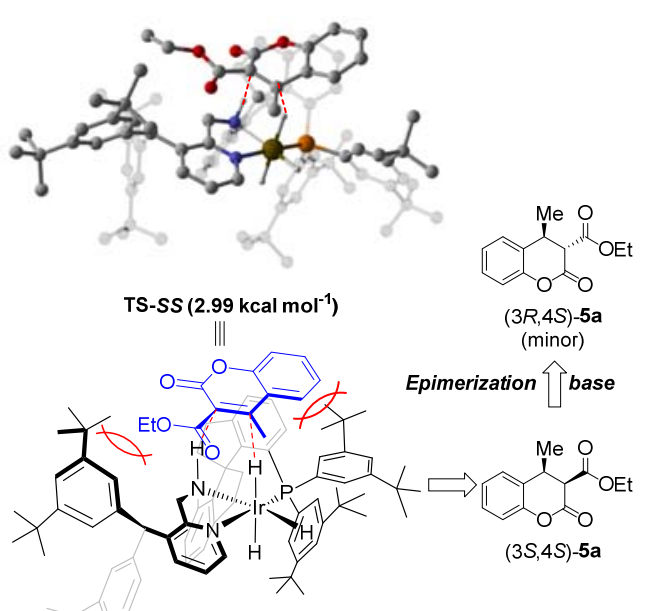

unfavorable

Figure S1. The transition state structures for the $\mathrm{H}^{-} / \mathrm{H}^{+}$transfer to $4 \mathrm{a}$

For the model of the interaction between the substrate $\mathbf{4 a}$ and catalyst, the Boltzmann analysis of the two competing TS structures were summarized (Table S2). The calculation of the ee value based on the Boltzmann distribution for each TS structure indicated that TS-RR (down-face attack) is preferable than TS-SS (upper-face attack), which was found lying $2.99 \mathrm{kcal} / \mathrm{mol}$ in free activation energy, leading to formation of $(3 R, 4 R)-\mathbf{5 a}$ as the major product (under basic conditions, epimerization at 3-position of the substrate occurs and formed $(3 S, 4 R)-\mathbf{5 a}$ as a stable structure eventually) with a calculated $98 \%$ ee, which are in good agreement with the experimental results $(95 \%$ ee).

Table S2. DFT-calculated Boltzmann distribution of the TS structures for $(R)-\mathrm{Ir}-\mathrm{H}$ catalyzed hydride/proton transfer to $\mathbf{4 a}$

\begin{tabular}{lll}
\hline Transition States & Free energy in solution (a.u.) & relative G $(\mathrm{kcal} / \mathrm{mol})$ \\
\hline TS-SS & -4468.33141 & 2.99306 \\
TS-RR & -4468.33618 & 0.00000 \\
\hline & Ee $(\%)$ & 0.98732 \\
\hline
\end{tabular}




\section{References}

(1) Ukrainets, I. V.; Sidorenko, L. V.; Gorokhova, O. V.; Shishkina, S. V. 4-Hydroxy-2-quinolones. 96*. Synthesis and Properties of 4-Methyl-2-oxo-1,2-dihydroquinoline-3-carboxylic Acid. Chem. Heterocycl. Com+. 2006, 42, 776 .

(2) Hur, W.; Sun, Z.; Jiang, T.; Mason, D. E.; Peters, E. C.; Zhang, D. D.; Luesch, H.; Schultz, P. G.; Gray, N. S. A Small-Molecule Inducer of the Antioxidant Response Element. Chem. Biol. (Cambridge, MA, U. S.) 2010, 17, 537.

(3) Arcadi, A.; Cacchi, S.; Fabrizi, G.; Manna, F.; Pace, P. ortho-Ethynylmalonmonoanilide et Ester as a Useful Building Block for the Preparation of 3,4-Disubstituted 2(1H)-quinolones and of 3,4Disubstituted and 2,3,4-Trisubstituted Quinolines. Synlett 1998, 446.

(4) Bui, C. T. One-pot Microwave-assisted Synthesis of 3,4-Disubstituted 2-Quinolinones. Synth. Commun. 2014, 44, 1122.

(5) Soleiman, H. A. New Route Synthesis of Some Quinolin-2-one Derivatives. Chem. Pap. 2004, 58, 330 .

(6) Carrër, A.; Brion, J.-D.; Messaoudi, S.; Alami, M. Synthesis of 3,4-Disubstituted Quinolin-2-(1H)ones via Palladium-Catalyzed Decarboxylative Arylation Reactions. Adv. Synth. Catal. 2013, 355, 2044.

(7) Ukrainets, I. V.; Gorokhova, O. V.; Sidorenko, L. V.; Bereznyakova, N. L. 4-Hydroxy-2-quinolones. Part 111. Simple Synthesis of 1-Substituted 4-Methyl-2-oxo-1,2-dihydroquinoline-3-carboxylic Acids. Chem. Heterocycl. Compd. (N. Y., NY, U. S.) 2007, 43, 58.

(8) Chung, S. J.; Joo, K. C.; Kim, D. H. Convenient Synthesis of 6-Substituted-2-chloro-5, 12-dihydro5-oxobenzoxazolo[3,2-a]quinolines and N-Acylated-3-chlorodibenz[b,e][1,4]oxazepin-11(5H)-ones. $J$. Heterocycl. Chem. 1997, 34, 485.

(9) Loh, C. C. J.; Schmid, M.; Peters, B.; Fang, X.; Lautens, M. Exploiting Distal Reactivity of Coumarins: A Rhodium-Catalyzed Vinylogous Asymmetric Ring-Opening Reaction. Angew. Chem. Int. Ed. 2016, 55, 4600.

(10) Dean, F. M.; Park, B. K. Activating Groups for the Ring Expansion of Coumarin by Diazoethane: Benzoyl, Pivaloyl, Arylsulfonyl, Arylsulfinyl, and Nitro. J. Chem. Soc., Perkin Trans. 1 1976, 1260.

(11) Tasqeeruddin, S.; Asiri, Y. I.; Shaheen, S. Zirconium(IV) Oxychloride: A Simple and Efficient Catalyst for the Synthesis of Chromen-2-one Derivatives. Asian J. Chem. 2020, 32, 2611.

(12) Zhang, L.; Qureshi, Z.; Sonaglia, L.; Lautens, M., Sequential Rhodium/Palladium Catalysis: Enantioselective Formation of Dihydroquinolinones in the Presence of Achiral and Chiral Ligands. Angew. Chem. Int. Ed. 2014, 53, 13850.

(13) Tang, X.; Blake, A. J.; Lewis, W.; Woodward, S. Asymmetric Conjugate Additions to 1,1'Diactivated Cyclic Enones-A Comparative Study. Tetrahedron: Asymmetry 2009, 20, 1881.

(14) Tripathi, K. N.; Belal, M.; Singh, R. P. Organo Photoinduced Decarboxylative Alkylation of Coumarins with N-(Acyloxy)phthalimide. J. Org. Chem. 2020, 85, 1193.

(15) vanLeeuwen, T.; Buzzetti, L.; Perego, L. A.; Melchiorre, P. A Redox-Active Nickel Complex that Acts as an Electron Mediator in Photochemical Giese Reactions. Angew. Chem. Int. Ed. 2019, 58, 4953.

(16) Patra, A.; Misra, S. K. Synthesis of 4-Alkylcoumarins. Indian J. Chem., Sect. B 1988, 27 B, 272.

(17) Murakami, M.; Kobayashi, K.; Hirai, K. Enantioselective Synthesis of the Key Intermediate of the Acyl-CoA: Cholesterol Acyltransferase (ACAT) Inhibitor (R-106578) Using 2,2'Bis(diphenylphosphino)-1,1'-binaphthyl (BINAP)-Ru(OAc) $)_{2}$ as a Catalyst. Chem. Pharm. Bull. 2000, 48, 1567.

(18) Wang, J.; Zhu, Z.-H.; Chen, M.-W.; Chen, Q.-A.; Zhou, Y.-G. Catalytic Biomimetic Asymmetric 
Reduction of Alkenes and Imines Enabled by Chiral and Regenerable NAD(P)H Models. Angew. Chem., Int. Ed. 2019, 58, 1813.

(19) Porter, M. R.; Xiao, H.; Wang, J.; Smith, S. B.; Topczewski, J. J. 3-Amino-chromanes and Tetrahydroquinolines as Selective 5- $\mathrm{HT}_{2 \mathrm{~B}}, 5-\mathrm{HT}_{7}$, or $\sigma_{1}$ Receptor Ligands. ACS Med. Chem. Lett. 2019, $10,1436$.

(20) Frisch, M. J.; Trucks, G. W.; Schlegel, H. B.; Scuseria, G. E.; Robb, M. A.; Cheeseman, J. R.; Scalmani, G.; Barone, V.; Petersson, G. A.; Nakatsuji, H.; Li, X.; Caricato, M.; Marenich, A. V.; Bloino, J.; Janesko, B. G.; Gomperts, R.; Mennucci, B.; Hratchian, H. P.; Ortiz, J. V.; Izmaylov, A. F.; Sonnenberg, J. L.; Williams-Young, F.; Ding, F.; Lipparini, F.; Egidi, F.; Goings, J.; Peng, B.; Petrone, A.; Henderson, T.; Ranasinghe, D.; Zakrzewski, V. G.; Gao, J.; Rega, N.; Zheng, G.; Liang, W.; Hada, M.; Ehara, M.; Toyota, K.; Fukuda, R.; Hasegawa, J.; Ishida, M.; Nakajima, T.; Honda, Y.; Kitao, O.; Nakai, H.; Vreven, T.; Throssell, K.; Montgomery Jr., J. A.; Peralta, J. E.; Ogliaro, F.; Bearpark, M. J.; Heyd, J. J.; Brothers, E. N.; Kudin, K. N.; Staroverov, V. N.; Keith, T. A.; Kobayashi, R.; Normand, J.; Raghavachari, K.; Rendell, A. P.; Burant, J. C.; Iyengar, S. S.; Tomasi, J.; Cossi, M.; Millam, J. M.; Klene, M.; Adamo, C.; Cammi, R.; Ochterski, J. W.; Martin, R. L.; Morokuma, K.; Farkas, O.; Foresman, J. B.; Fox, D. J. Gaussian 16, Revision A.03; Wallingford, CT, 2016.

(21) (a) Lee, C.; Yang, W.; Parr, R. G. Development of the Colle-Salvetti Correlation-Energy Formula into a Functional of the Electron density. Phys. Rev. B. 1988, 37, 785. (b) Becke, A. D. A New Mixing of Hartree-Fock and Local Density-functional theories. J. Chem. Phys. 1993, 98, 1372. (c) Becke, A. D. Density Functional Thermochemistry. III. The Role of Exact Exchange. J. Chem. Phys. 1993, 98, 5648. (22) Grimme, S.; Antony, J.; Ehrlich, S.; Krieg, H. A Consistent and Accurate ab Initio Parametrization of Density Functional Dispersion Correction (DFT-D) for the 94 Elements H-Pu. J. Chem. Phys. 2010, 132, 154104.

(23) Legault, C. Y. CYLview, 1.0b (Université de Sherbrooke, 2009). 
(E) NMR Spectra of New Compounds

Ethyl 4-methyl-2-oxo-1,2-dihydroquinoline-3-carboxylate (S1a)

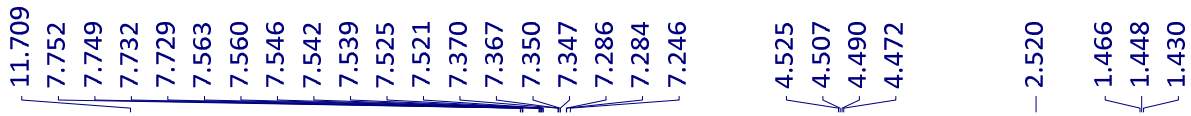<smiles>CCOC(=O)c1c(C)c2ccccc2[nH]c1=O</smiles>

S1a

$\left({ }^{1} \mathrm{H}\right.$ NMR, $\left.400 \mathrm{MHz}, \mathrm{CDCl}_{3}\right)$

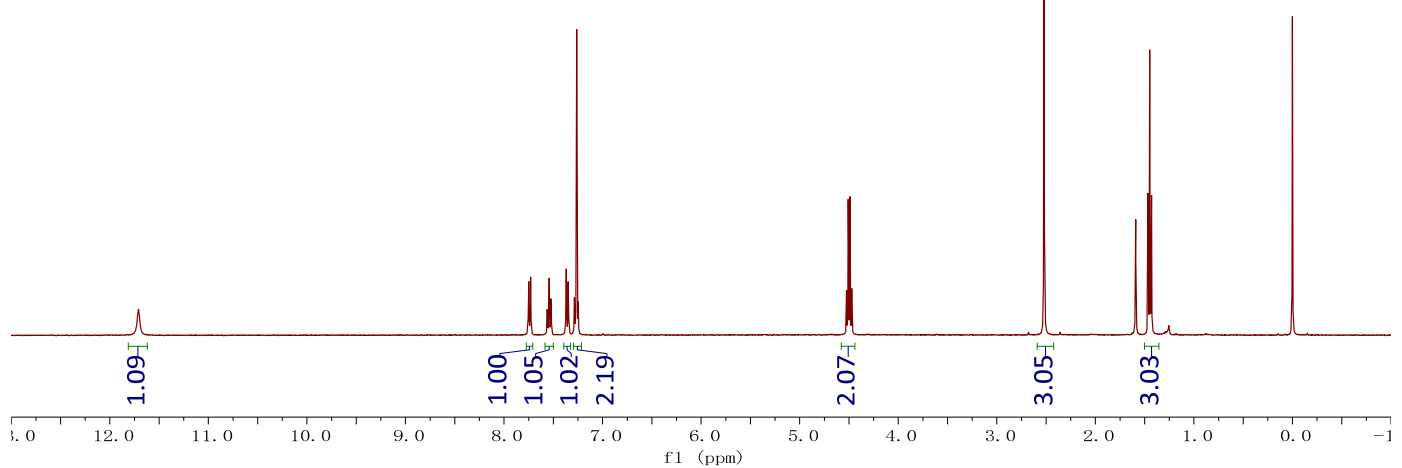

Ethyl 4-ethyl-2-oxo-1,2-dihydroquinoline-3-carboxylate (S1b)

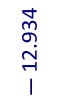

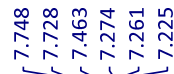

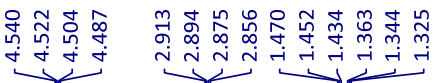<smiles>CCOC(=O)c1c(CC)c2ccccc2[nH]c1=O</smiles>

S1b

$\left({ }^{1} \mathrm{H} \mathrm{NMR}, 400 \mathrm{MHz}, \mathrm{CDCl}_{3}\right)$

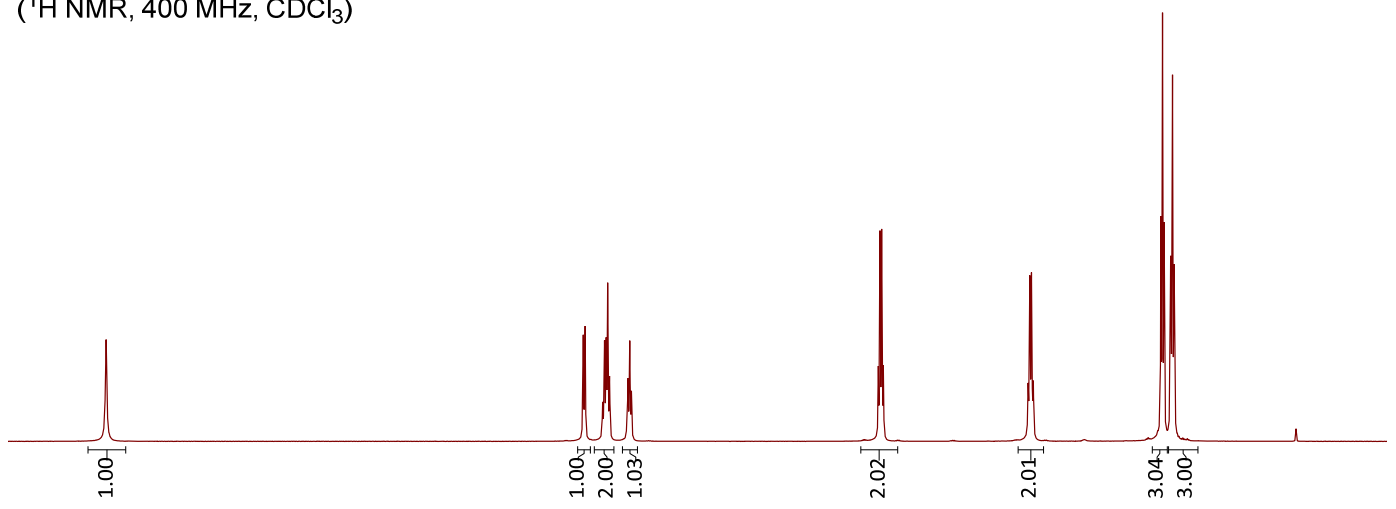

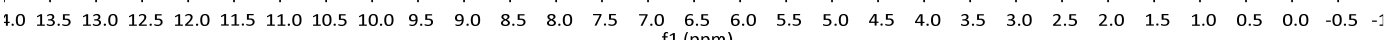
f1 (ppm) 

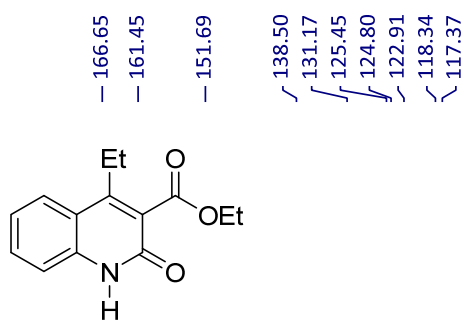

S1b

$\left({ }^{13} \mathrm{C} \mathrm{NMR}, 101 \mathrm{MHz}, \mathrm{CDCl}_{3}\right)$
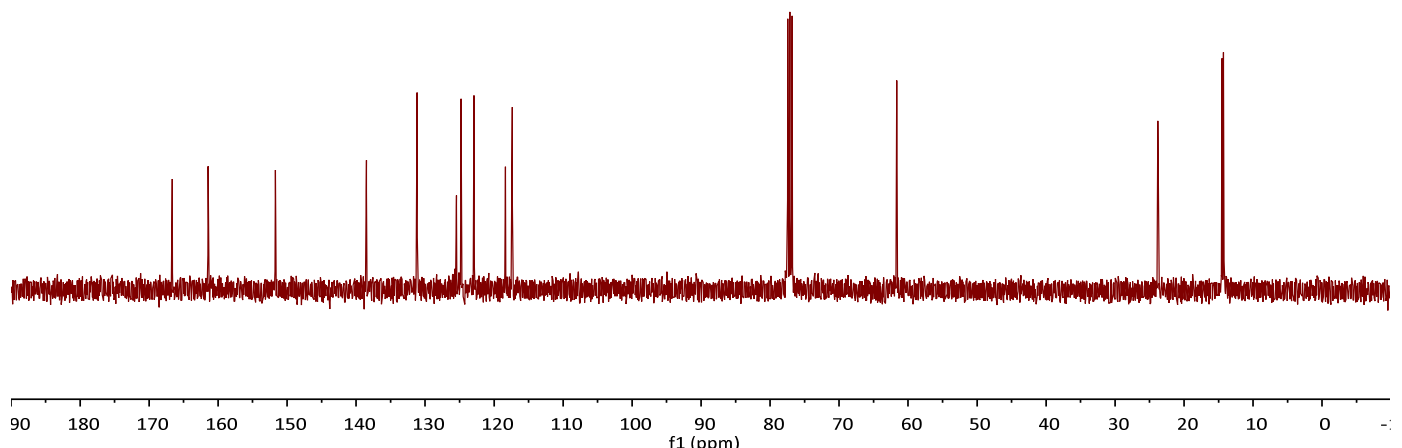

Ethyl 2-oxo-4-propyl-1,2-dihydroquinoline-3-carboxylate (S1c)

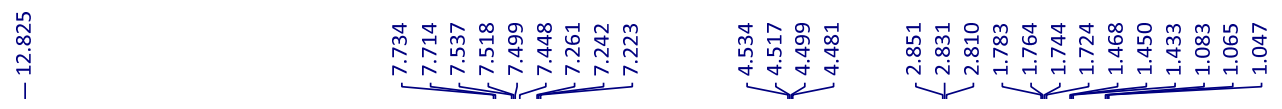<smiles>CCOC(=O)c1c(C(C)C)c2ccccc2[nH]c1=O</smiles>

S1c

$\left({ }^{1} \mathrm{H} \mathrm{NMR}, 400 \mathrm{MHz}, \mathrm{CDCl}_{3}\right)$

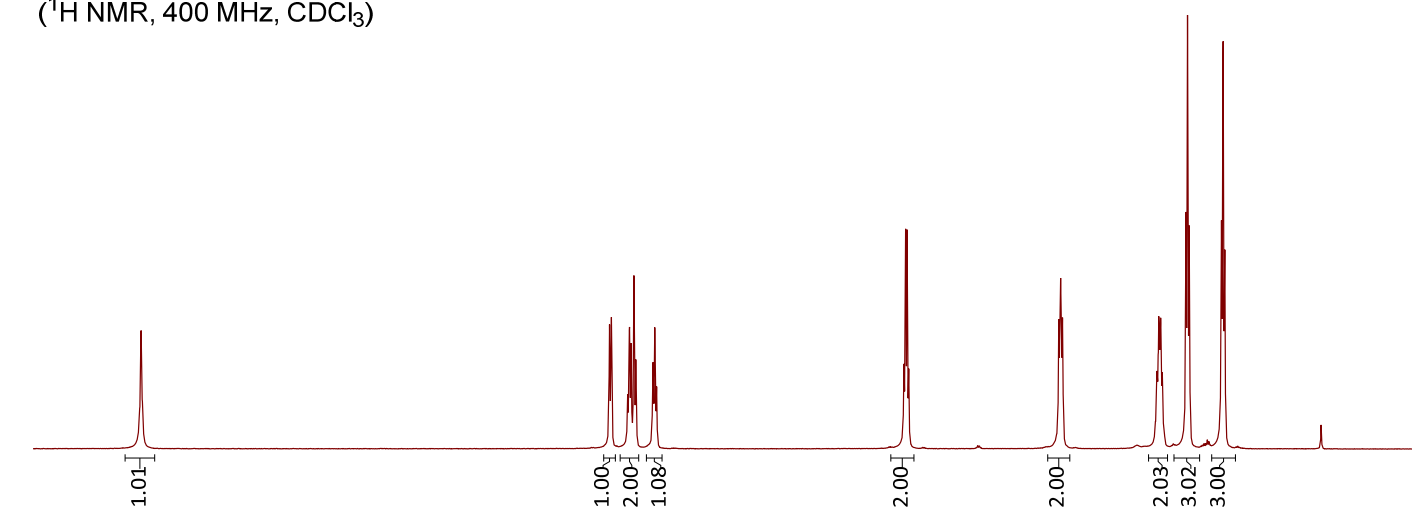

$\begin{array}{lllllllllllllllllllllllllllllllll}1.0 & 13.5 & 13.0 & 12.5 & 12.0 & 11.5 & 11.0 & 10.5 & 10.0 & 9.5 & 9.0 & 8.5 & 8.0 & 7.5 & 7.0 & 6.5 & 6.0 & 5.5 & 5.0 & 4.5 & 4.0 & 3.5 & 3.0 & 2.5 & 2.0 & 1.5 & 1.0 & 0.5 & 0.0 & -0.5 & -1\end{array}$ 


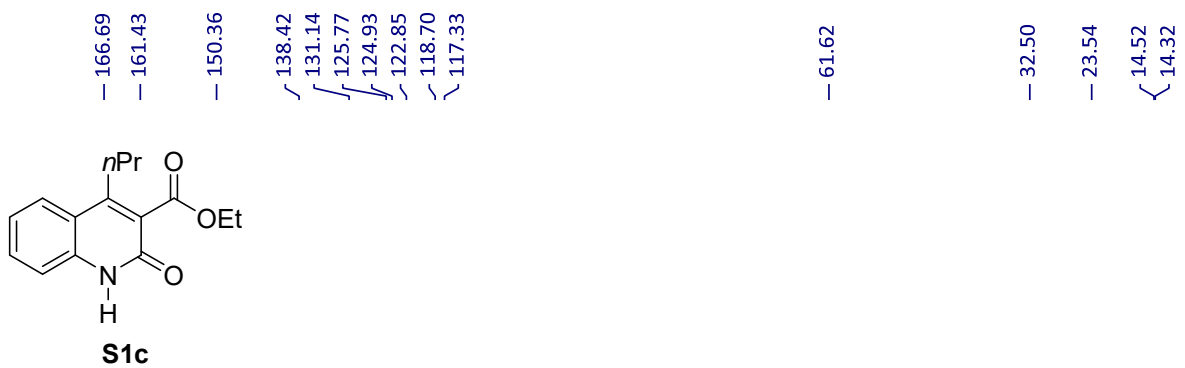

$\left({ }^{13} \mathrm{C}\right.$ NMR, $\left.101 \mathrm{MHz}, \mathrm{CDCl}_{3}\right)$
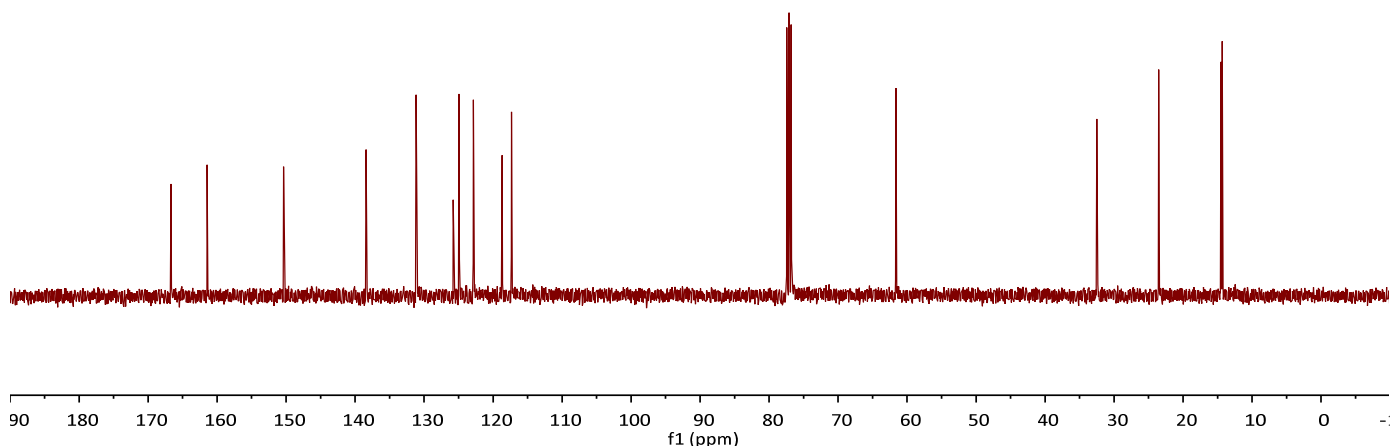

Ethyl 4-isobutyl-2-oxo-1,2-dihydroquinoline-3-carboxylate (S1d)

용

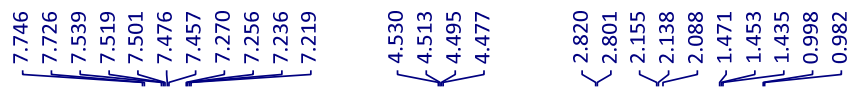<smiles>CCOC(=O)c1c(CC(C)C)c2ccccc2[nH]c1=O</smiles>

S1d

$\left({ }^{1} \mathrm{HNMR}, 400 \mathrm{MHz}, \mathrm{CDCl}_{3}\right)$

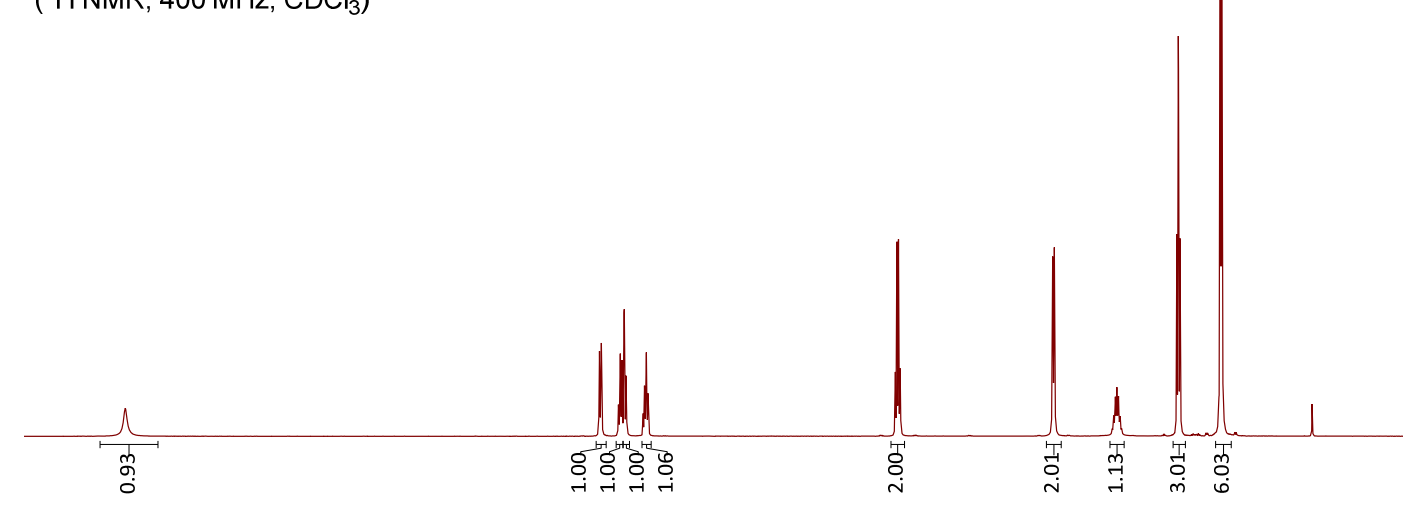

$\begin{array}{lllllllllllllllllllllllllllllllllllllllll}13.5 & 13.0 & 12.5 & 12.0 & 11.5 & 11.0 & 10.5 & 10.0 & 9.5 & 9.0 & 8.5 & 8.0 & 7.5 & 7.0 & 6.5 & 6.0 & 5.5 & 5.0 & 4.5 & 4.0 & 3.5 & 3.0 & 2.5 & 2.0 & 1.5 & 1.0 & 0.5 & 0.0 & -0.5\end{array}$ 


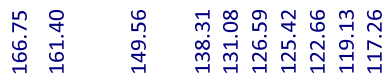

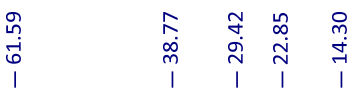

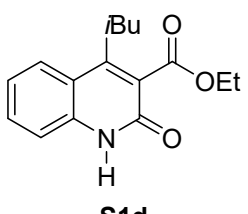

$\left({ }^{13} \mathrm{C} N M R, 101 \mathrm{MHz}, \mathrm{CDCl}_{3}\right)$
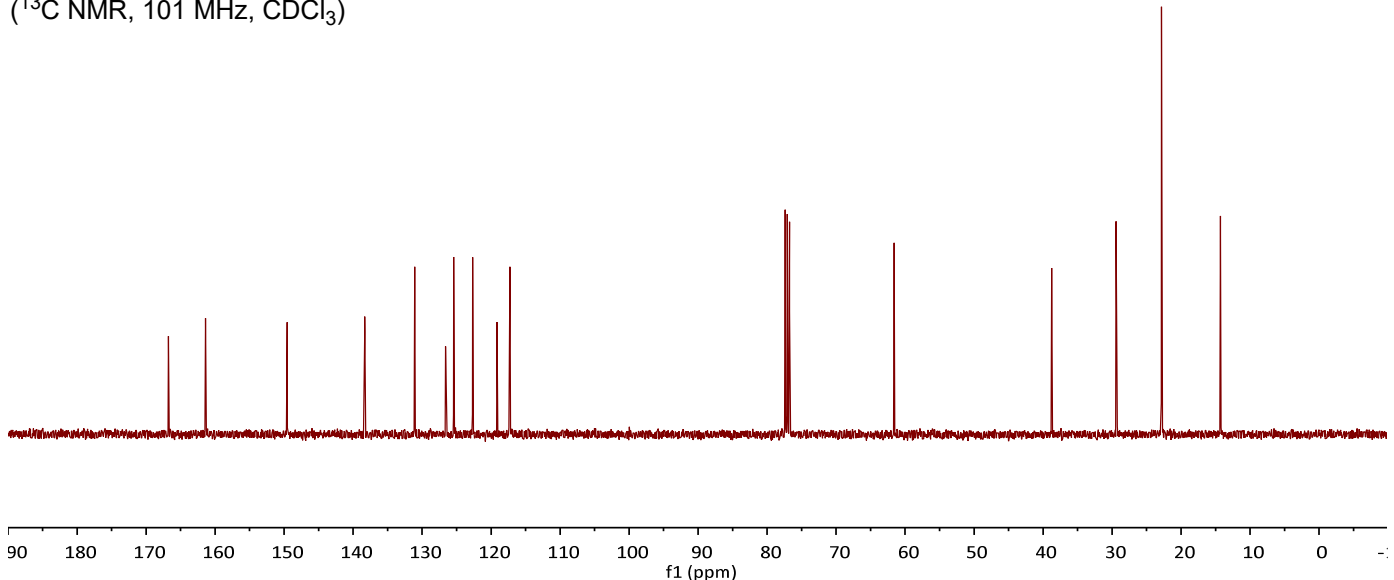

\section{Ethyl 4-benzyl-2-oxo-1,2-dihydroquinoline-3-carboxylate (S1e)}

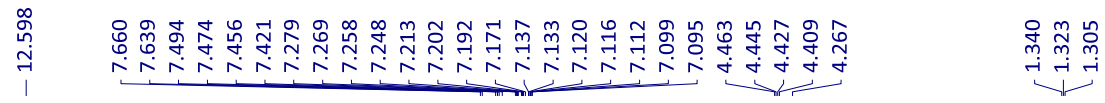<smiles>CCOC(=O)c1c(Cc2ccccc2)c2ccccc2[nH]c1=O</smiles>

S1e

( $\left.{ }^{1} \mathrm{H} \mathrm{NMR}, 400 \mathrm{MHz}, \mathrm{CDCl}_{3}\right)$

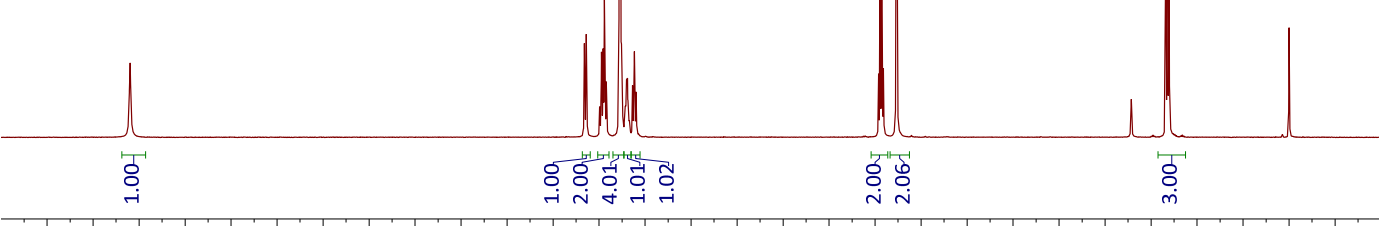

$\begin{array}{llllllllllllllllllllllllllllllllllllllll}13.5 & 13.0 & 12.5 & 12.0 & 11.5 & 11.0 & 10.5 & 10.0 & 9.5 & 9.0 & 8.5 & 8.0 & 7.5 & 7.0 & 6.5 & 6.0 & 5.5 & 5.0 & 4.5 & 4.0 & 3.5 & 3.0 & 2.5 & 2.0 & 1.5 & 1.0 & 0.5 & 0.0 & -0.5 & -1\end{array}$ 
Ethyl 2-oxo-4-phenethyl-1,2-dihydroquinoline-3-carboxylate (S1f)

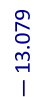

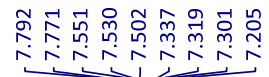

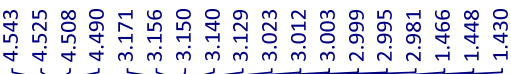<smiles>CCOC(=O)c1c(CCc2ccccc2)c2ccccc2[nH]c1=O</smiles>

S1f

( ${ }^{1} \mathrm{HNMR}, 400 \mathrm{MHz}, \mathrm{CDCl}_{3}$ )

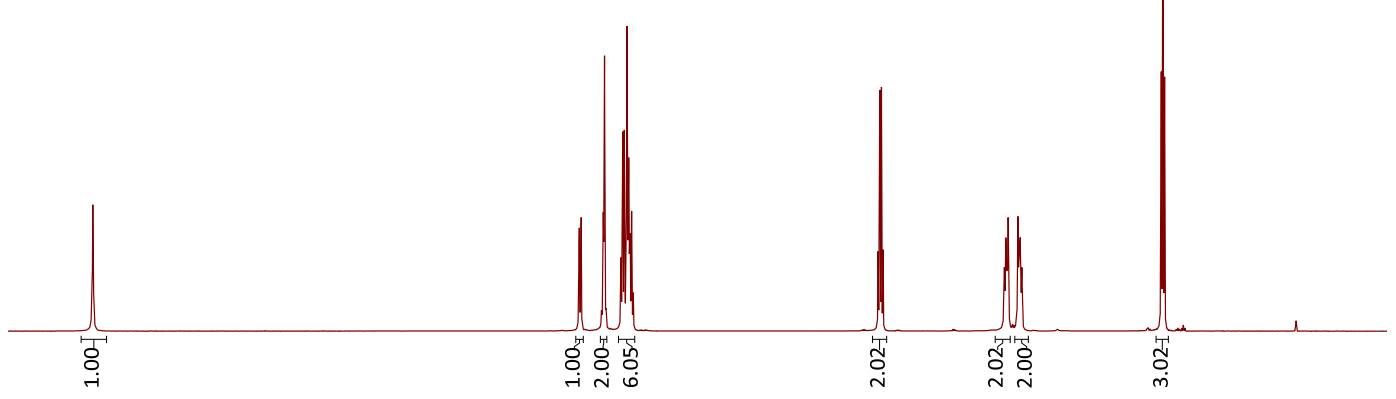

$\begin{array}{llllllllllllllllllllllllllllllll}13.5 & 13.0 & 12.5 & 12.0 & 11.5 & 11.0 & 10.5 & 10.0 & 9.5 & 9.0 & 8.5 & 8.0 & 7.5 & 7.0 & 6.5 & 6.0 & 5.5 & 5.0 & 4.5 & 4.0 & 3.5 & 3.0 & 2.5 & 2.0 & 1.5 & 1.0 & 0.5 & 0.0 & -0.5 & -1\end{array}$

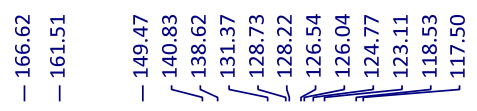

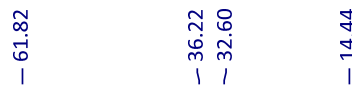

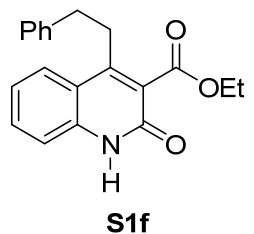

$\left({ }^{13} \mathrm{C} \mathrm{NMR}, 101 \mathrm{MHz}, \mathrm{CDCl}_{3}\right)$

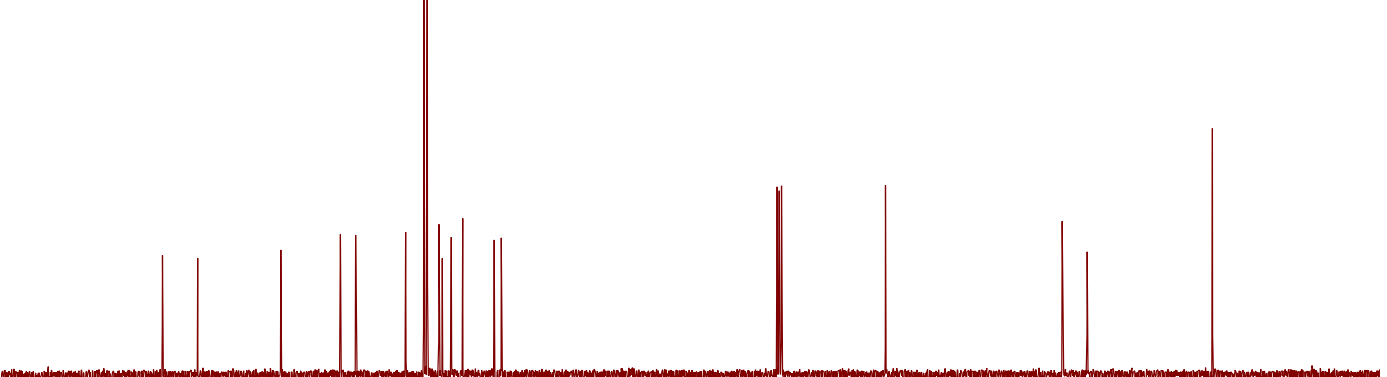

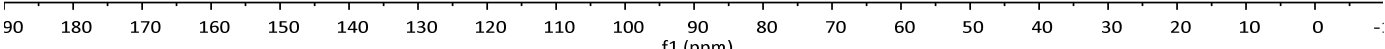


Ethyl 2-oxo-4-pentyl-1,2-dihydroquinoline-3-carboxylate (S1g)

$\stackrel{\circ}{\infty}$
$\stackrel{+}{+}$
1

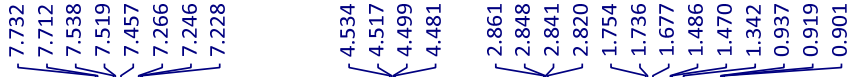<smiles>CCOC(=O)c1c(COC)c2ccccc2[nH]c1=O</smiles>

S1g

$\left({ }^{1} \mathrm{H} \mathrm{NMR}, 400 \mathrm{MHz}, \mathrm{CDCl}_{3}\right)$

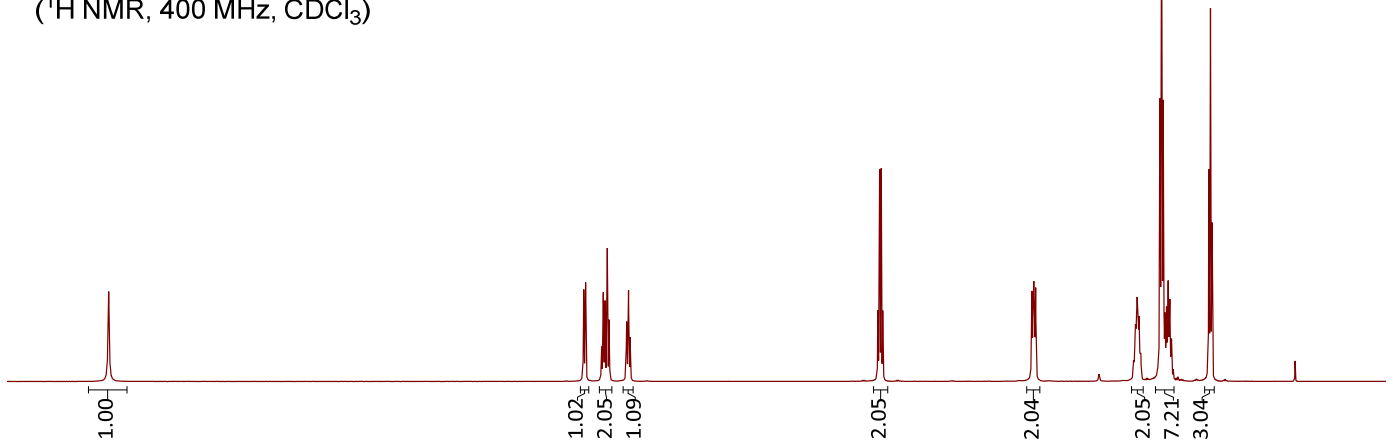

$\begin{array}{llllllllllllllllllllllllllllllll}13.5 & 13.0 & 12.5 & 12.0 & 11.5 & 11.0 & 10.5 & 10.0 & 9.5 & 9.0 & 8.5 & 8.0 & 7.5 & 7.0 & 6.5 & 1 & 6.0 & 5.5 & 5.0 & 4.5 & 4.0 & 3.5 & 3.0 & 2.5 & 2.0 & 1.5 & 1.0 & 0.5 & 0.0 & -0.5 & -1\end{array}$

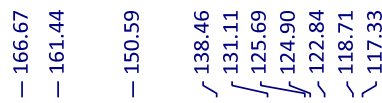

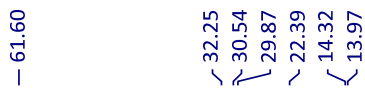<smiles>CCOC(=O)c1c(C)c2ccccc2[nH]c1=O</smiles>

S1g

$\left({ }^{13} \mathrm{C} \mathrm{NMR}, 101 \mathrm{MHz}, \mathrm{CDCl}_{3}\right)$

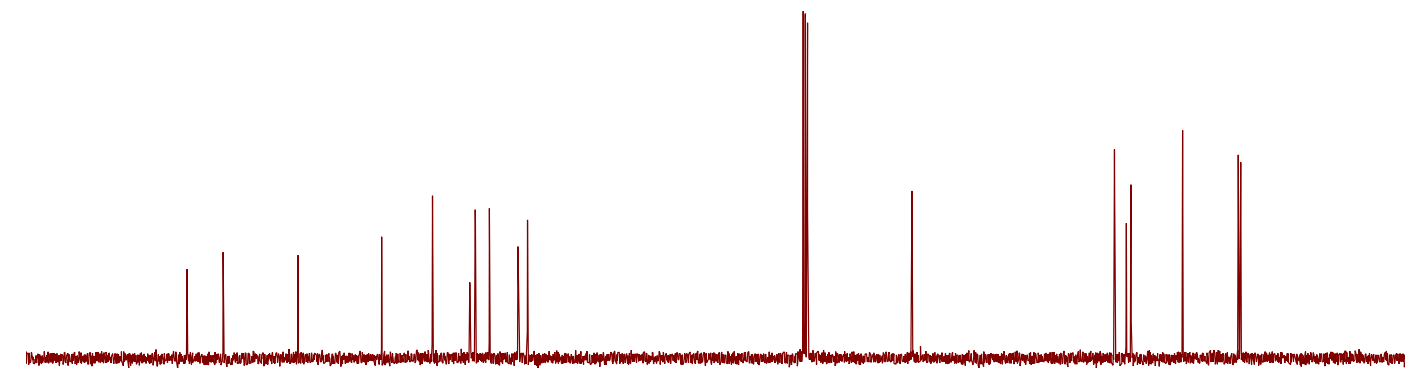

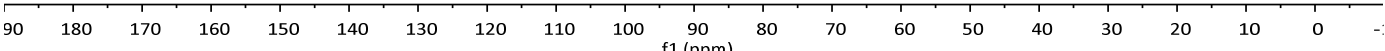


Ethyl 4-cyclopropyl-2-oxo-1,2-dihydroquinoline-3-carboxylate (S1h)

$\underset{\substack { 1 \\ \begin{subarray}{c}{n \\ \text { I }{ 1 \\ \begin{subarray} { c } { n \\ \text { I } } }\end{subarray}}{1}$

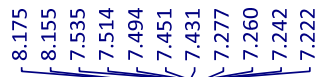

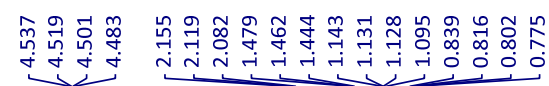<smiles>CCOC(=O)c1c(C2CC2)c2ccccc2[nH]c1=O</smiles>

S1h

$\left({ }^{1} \mathrm{H} \mathrm{NMR}, 400 \mathrm{MHz}, \mathrm{CDCl}_{3}\right)$

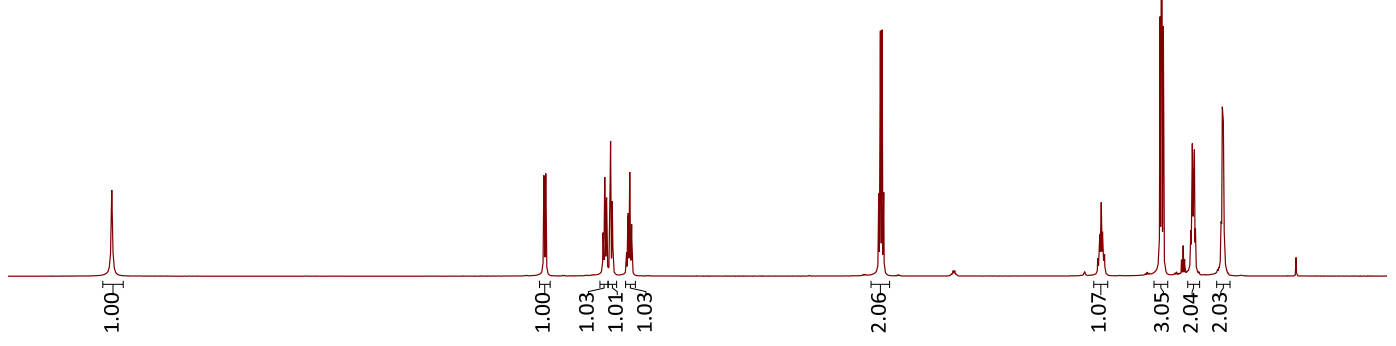

$\begin{array}{llllllllllllllllllllllllllllllllllll}13.5 & 13.0 & 12.5 & 12.0 & 11.5 & 11.0 & 10.5 & 10.0 & 9.5 & 9.0 & 8.5 & 8.0 & 7.5 & 7.0 & 6.5 & 6.0 & 5.5 & 5.0 & 4.5 & 4.0 & 3.5 & 3.0 & 2.5 & 2.0 & 1.5 & 1.0 & 0.5 & 0.0 & -0.5\end{array}$

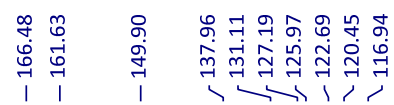
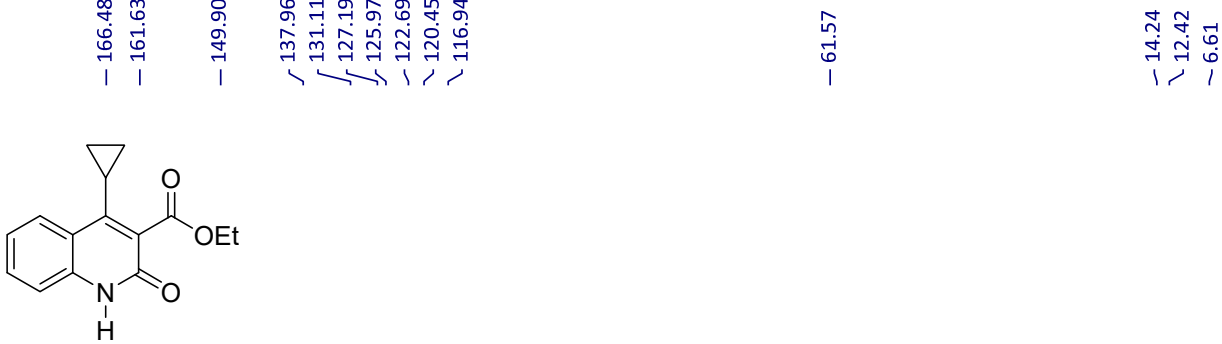

s1h

$\left({ }^{13} \mathrm{C}\right.$ NMR, $\left.101 \mathrm{MHz}, \mathrm{CDCl}_{3}\right)$
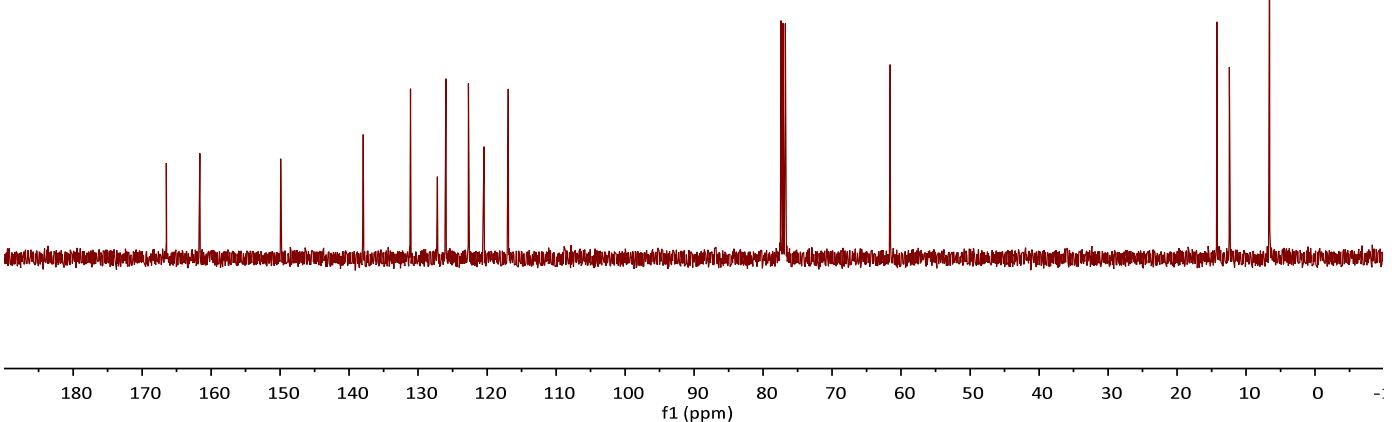
Ethyl 2-oxo-4-phenyl-1,2-dihydroquinoline-3-carboxylate (S1i)

ํㅗำ
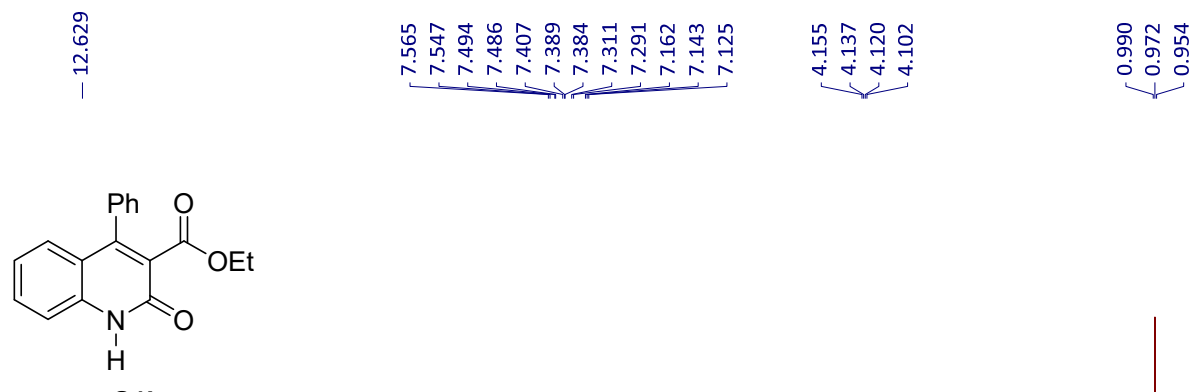

S1i

$\left({ }^{1} \mathrm{H}\right.$ NMR, $\left.400 \mathrm{MHz}, \mathrm{CDCl}_{3}\right)$

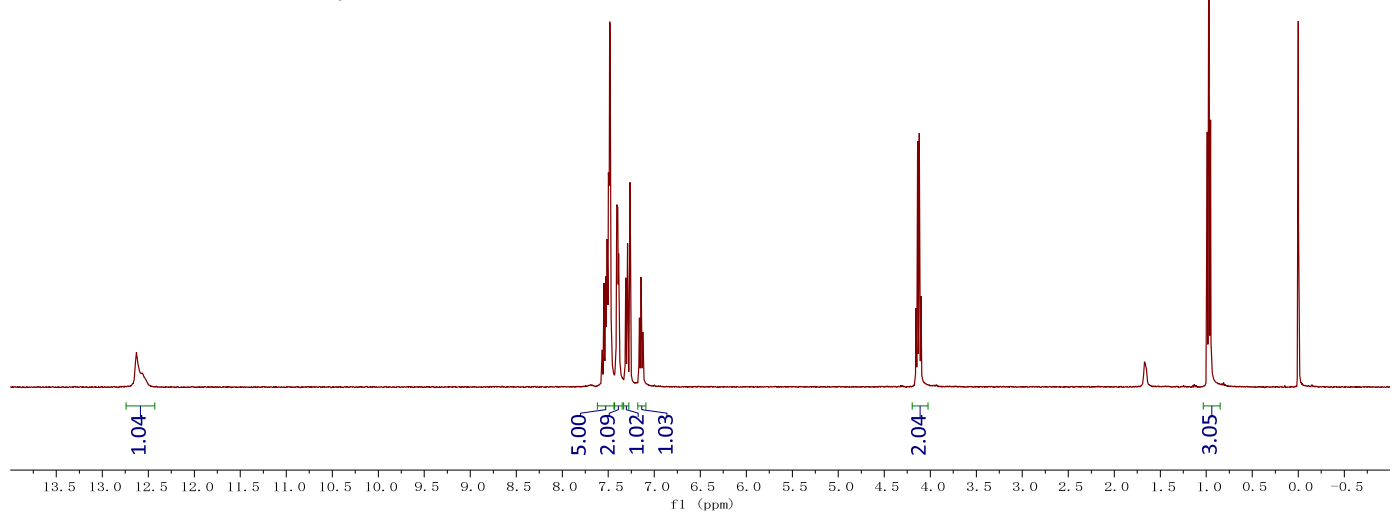

Ethyl 6-bromo-4-methyl-2-oxo-1,2-dihydroquinoline-3-carboxylate (S1j)

ث্ণ

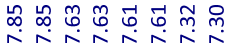

䓟赑导守

\)<smiles>CCOC(=O)c1c(C)c2cc(Br)ccc2[nH]c1=O</smiles>

S1j

$\left({ }^{1} \mathrm{H}\right.$ NMR, $\left.400 \mathrm{MHz}, \mathrm{CDCl}_{3}\right)$

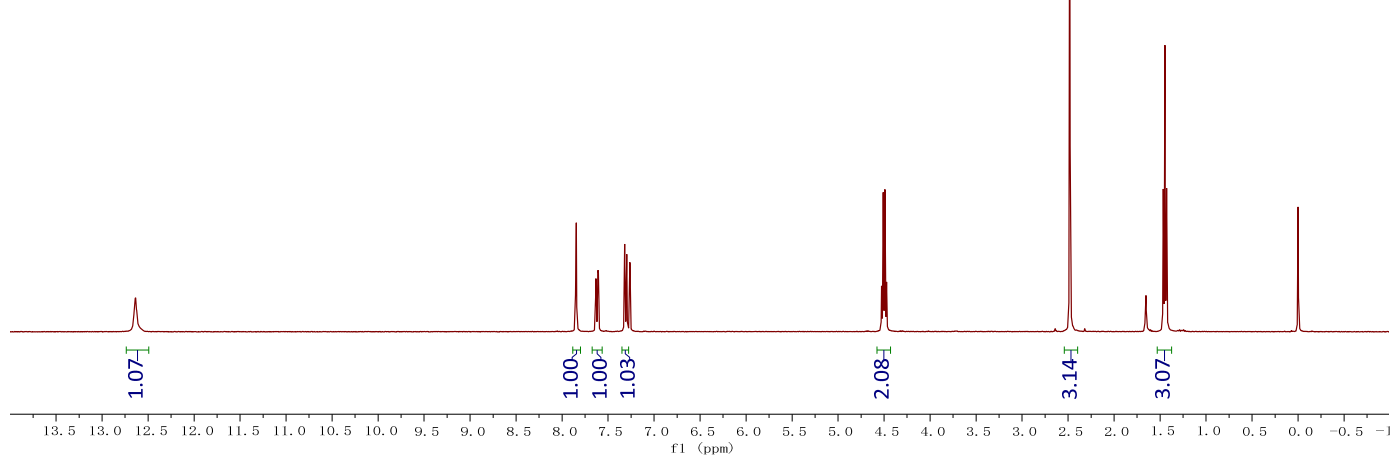


Ethyl 6-methoxy-4-methyl-2-oxo-1,2-dihydroquinoline-3-carboxylate (S1k)
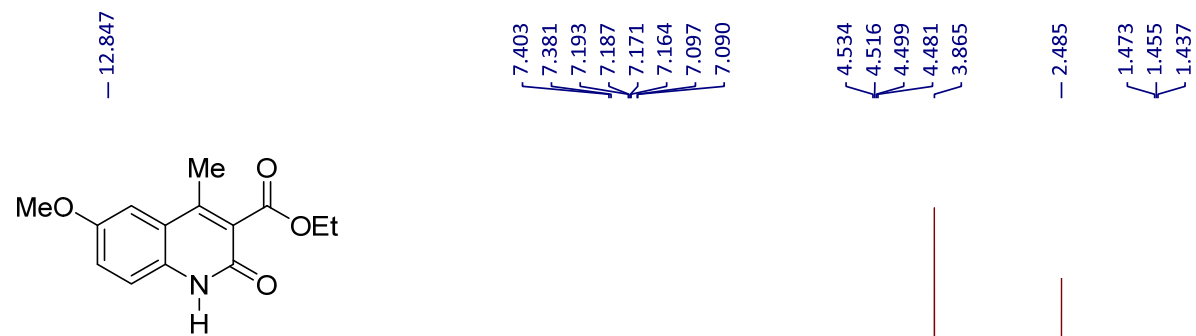

S1k

$\left({ }^{1} \mathrm{H} \mathrm{NMR}, 400 \mathrm{MHz}, \mathrm{CDCl}_{3}\right)$

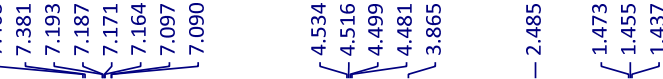

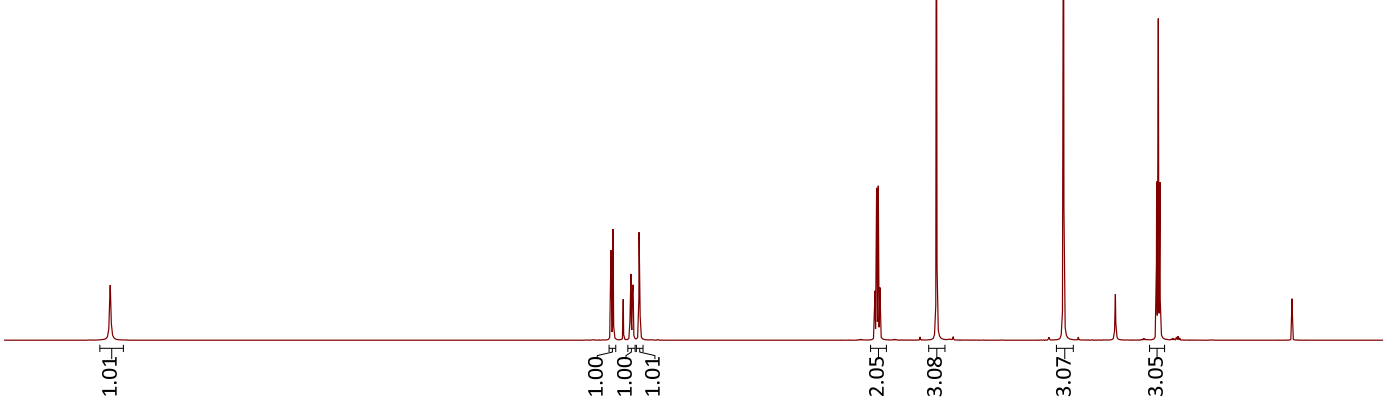

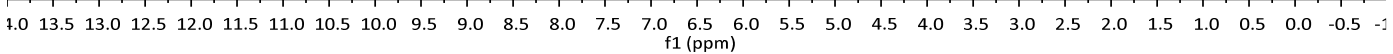

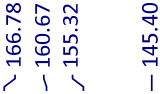

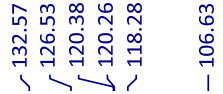

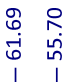

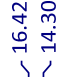

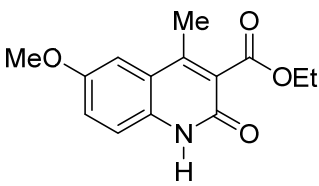

S1k

$\left({ }^{13} \mathrm{C}\right.$ NMR, $\left.101 \mathrm{MHz}, \mathrm{CDCl}_{3}\right)$

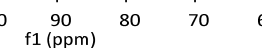

50

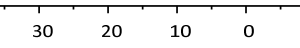


Ethyl 1,4-dimethyl-2-oxo-1,2-dihydroquinoline-3-carboxylate (2a)

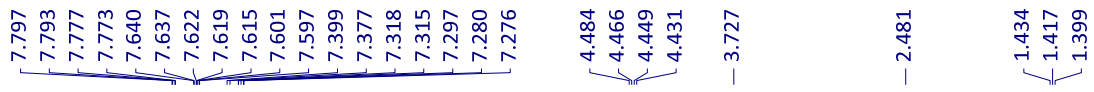

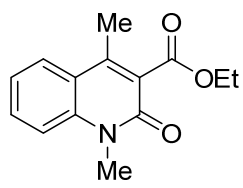

$2 a$

$\left({ }^{1} \mathrm{HNMR}, 400 \mathrm{MHz}, \mathrm{CDCl}_{3}\right)$

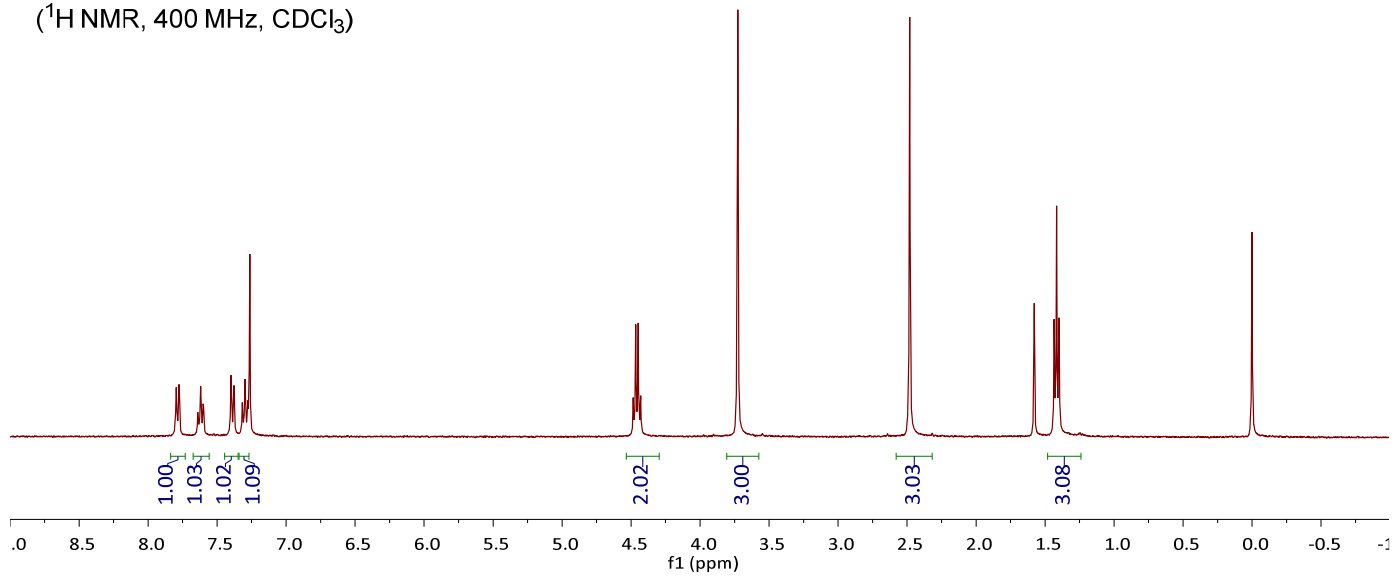

Ethyl 4-ethyl-1-methyl-2-oxo-1,2-dihydroquinoline-3-carboxylate (2b)

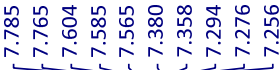

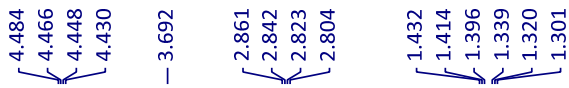

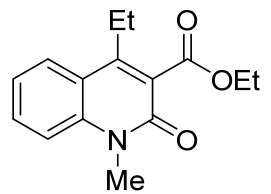

2b

$\left({ }^{1} \mathrm{H} \mathrm{NMR}, 400 \mathrm{MHz}, \mathrm{CDCl}_{3}\right)$

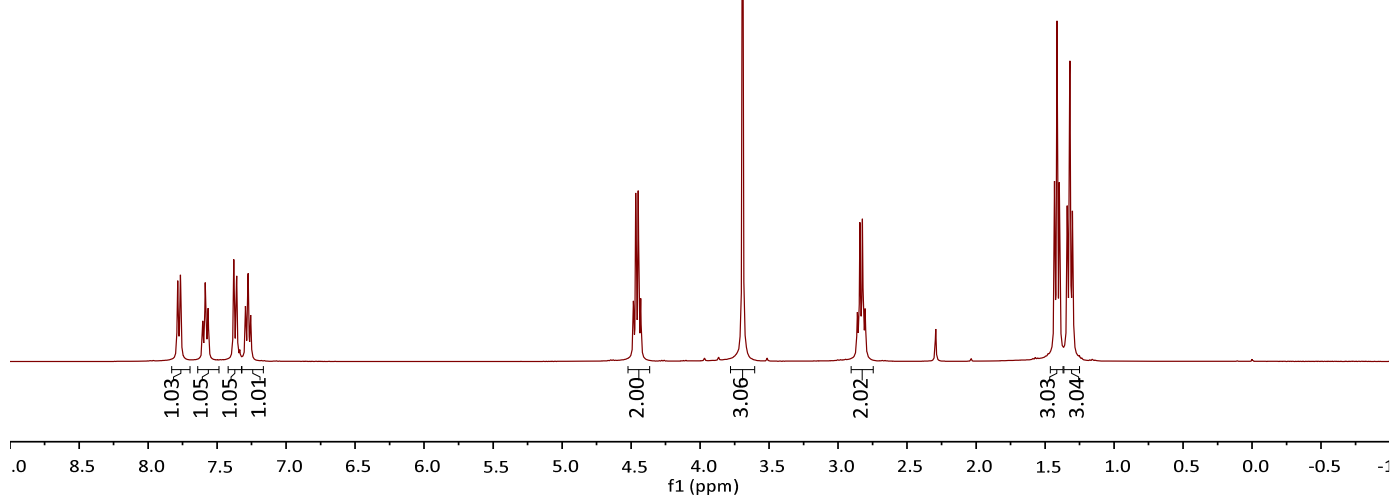




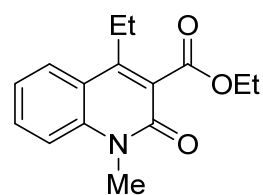

2b

$\left({ }^{13} \mathrm{C}\right.$ NMR, $\left.101 \mathrm{MHz}, \mathrm{CDCl}_{3}\right)$
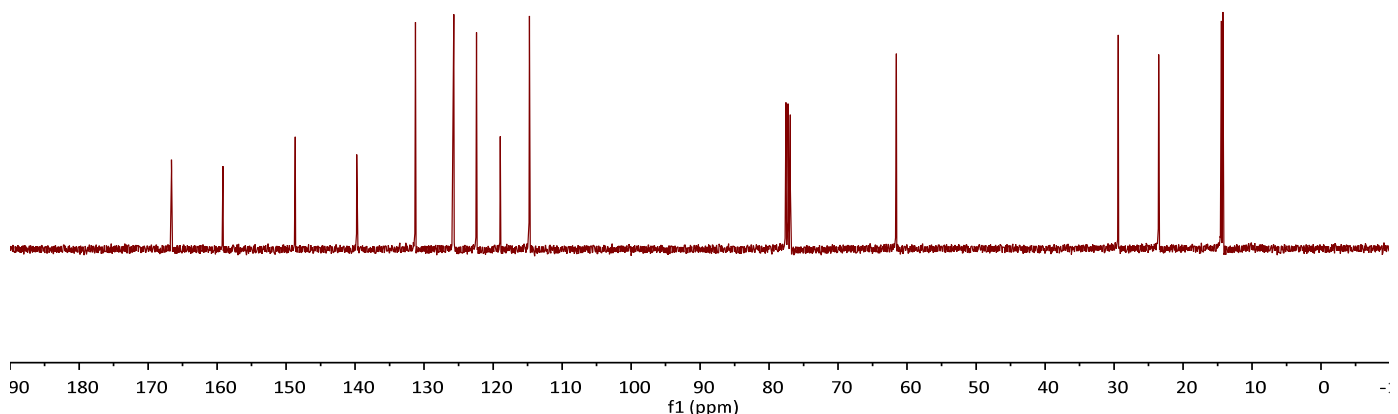

Ethyl 1-methyl-2-oxo-4-propyl-1,2-dihydroquinoline-3-carboxylate (2c)

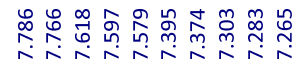

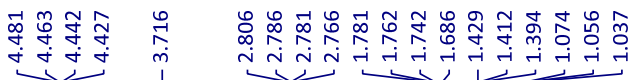

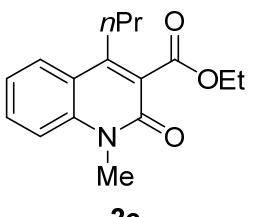

$\left({ }^{1} \mathrm{H} \mathrm{NMR}, 400 \mathrm{MHz}, \mathrm{CDCl}_{3}\right)$

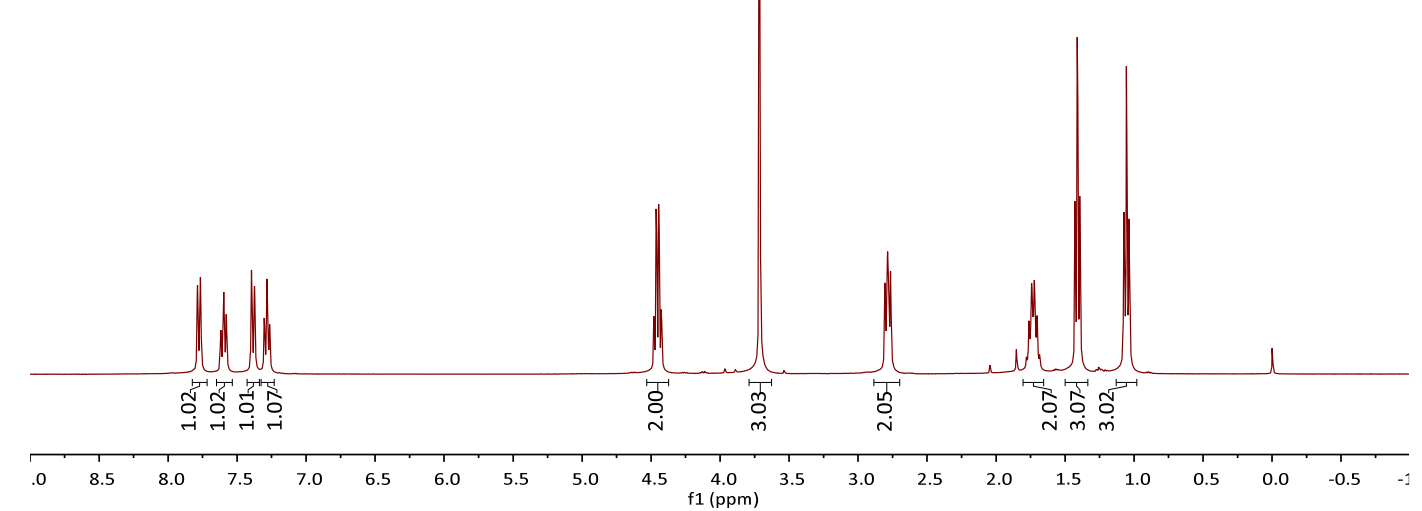




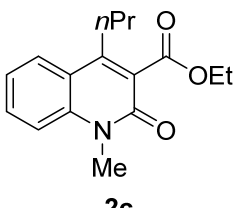

$\left({ }^{13} \mathrm{C}\right.$ NMR, $\left.101 \mathrm{MHz}, \mathrm{CDCl}_{3}\right)$
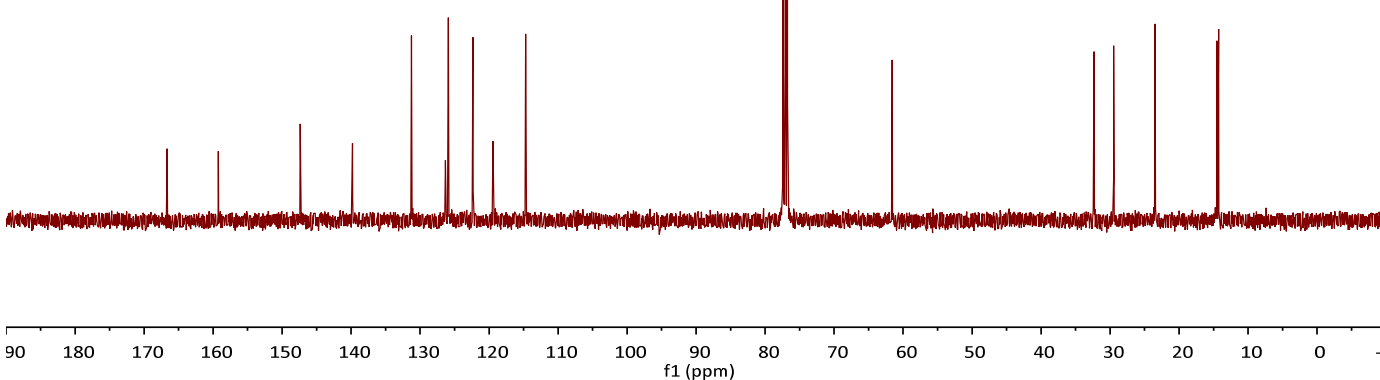

Ethyl 4-isobutyl-1-methyl-2-oxo-1,2-dihydroquinoline-3-carboxylate (2d)

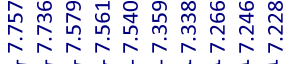

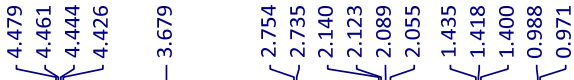

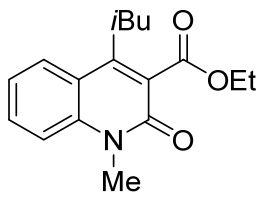

2d

$\left({ }^{1} \mathrm{H} \mathrm{NMR}, 400 \mathrm{MHz}, \mathrm{CDCl}_{3}\right)$

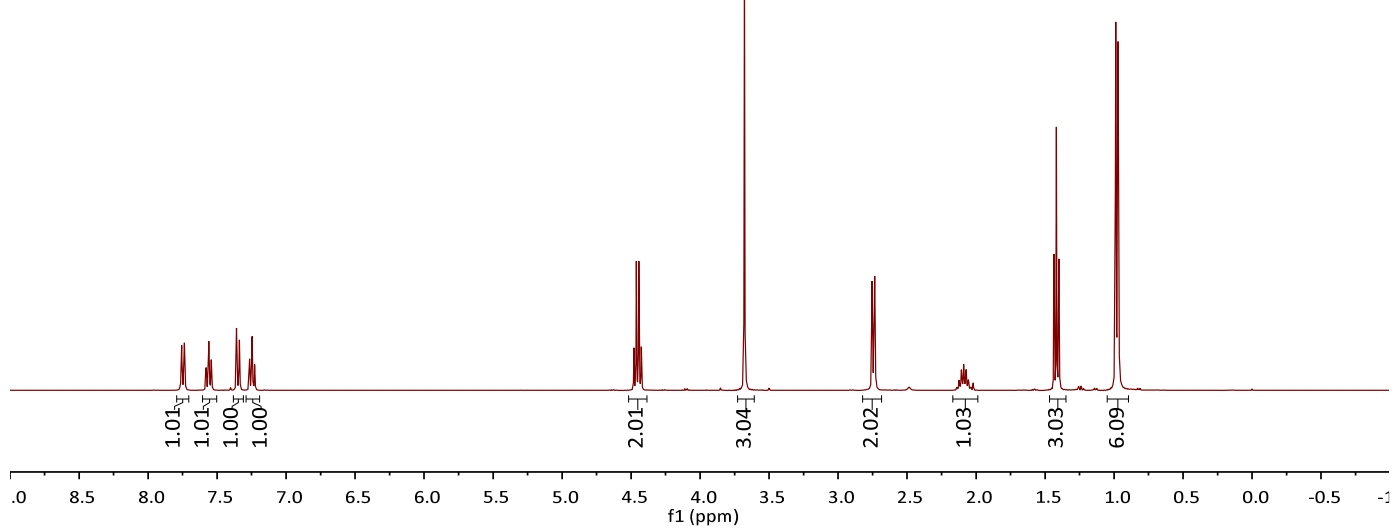




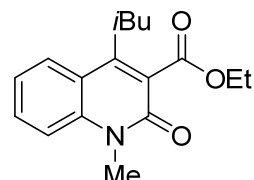

2d

$\left({ }^{13} \mathrm{C} \mathrm{NMR}, 101 \mathrm{MHz}, \mathrm{CDCl}_{3}\right)$

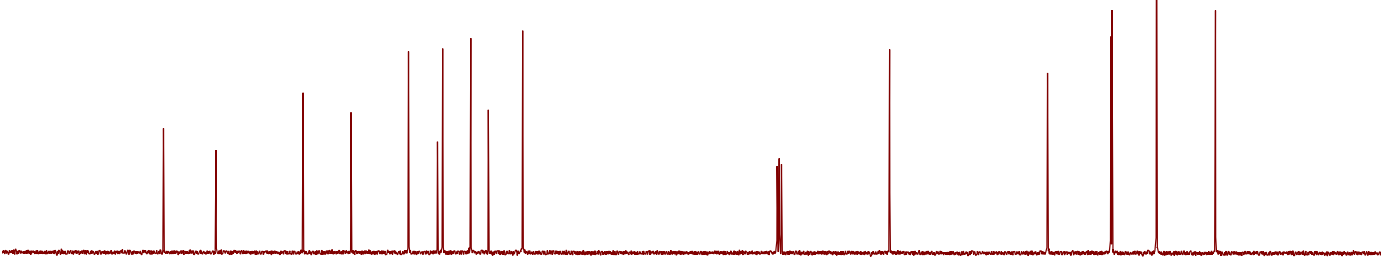

$\begin{array}{lllllllllllllllllll}180 & 170 & 160 & 150 & 140 & 130 & 120 & 110 & 100 & 90 & 80 & 70 & 60 & 50 & 40 & 30 & 20 & 10 & 0\end{array}$

Ethyl 4-benzyl-1-methyl-2-oxo-1,2-dihydroquinoline-3-carboxylate (2e)

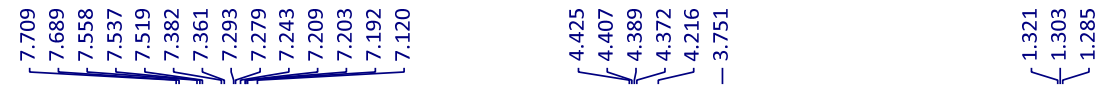<smiles>CCOC(=O)c1c(Cc2ccccc2)c2ccccc2n(C)c1=O</smiles>

$2 e$

('H NMR, $\left.400 \mathrm{MHz}, \mathrm{CDCl}_{3}\right)$

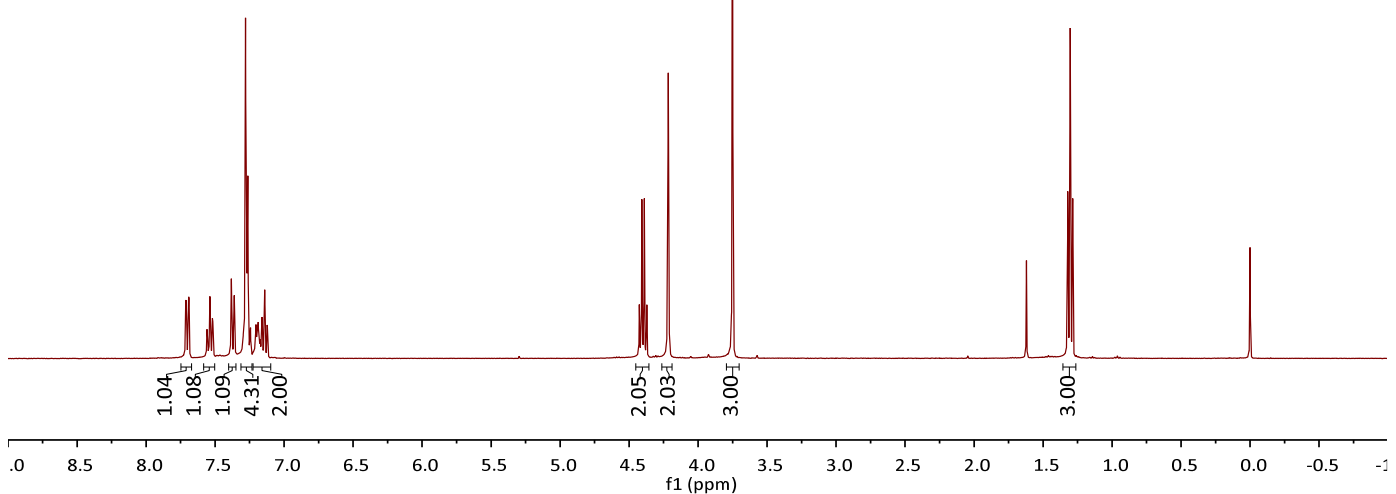


<smiles>CCOC(=O)c1c(Cc2ccccc2)c2ccccc2n(C)c1=O</smiles>

$2 \mathrm{e}$

$\left({ }^{13} \mathrm{C}\right.$ NMR, $\left.101 \mathrm{MHz}, \mathrm{CDCl}_{3}\right)$

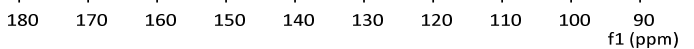

Ethyl 1-methyl-2-oxo-4-phenethyl-1,2-dihydroquinoline-3-carboxylate (2f)
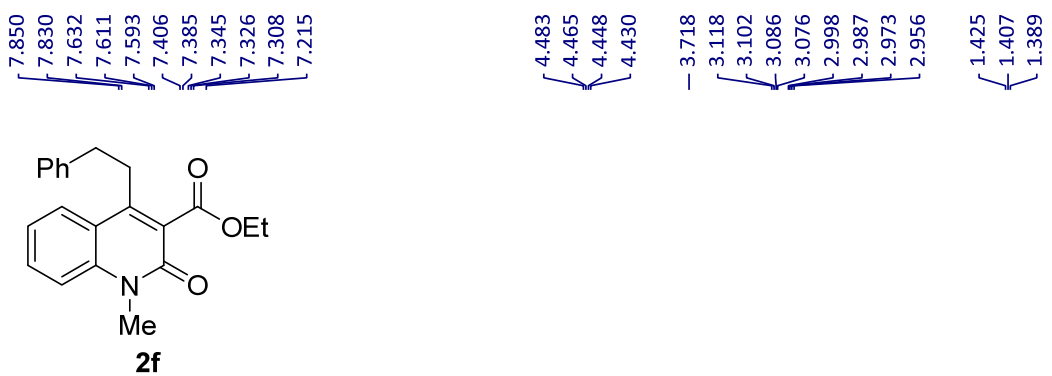

$\left({ }^{1} \mathrm{H} \mathrm{NMR}, 400 \mathrm{MHz}, \mathrm{CDCl}_{3}\right)$

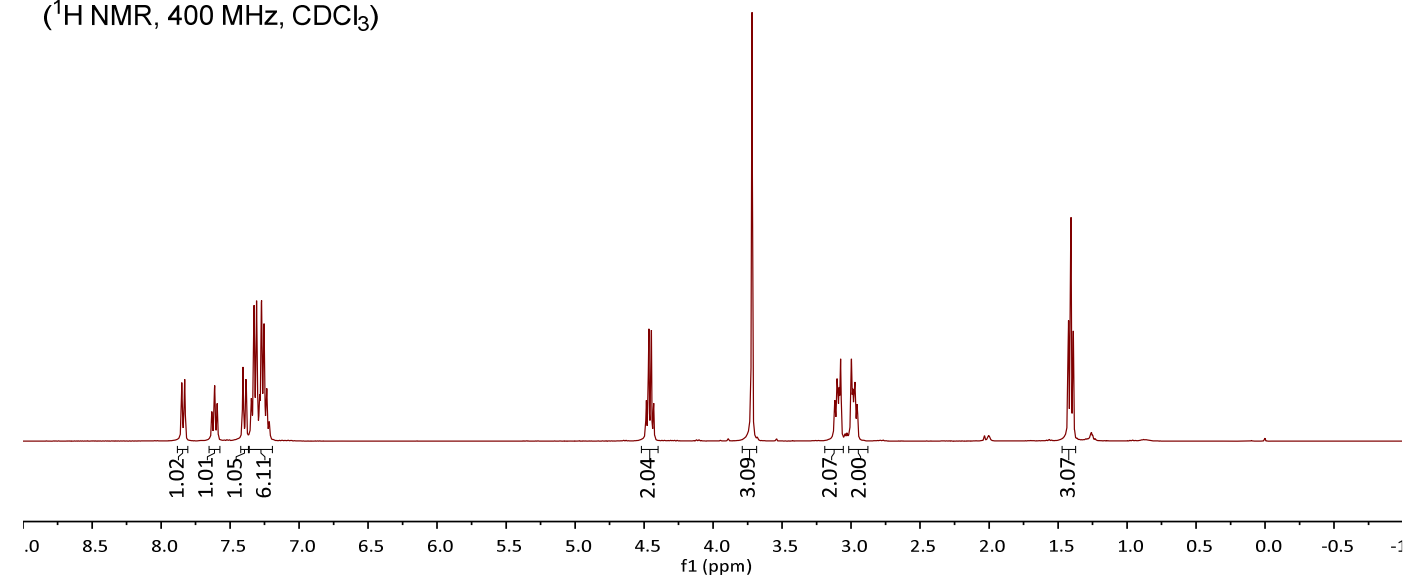




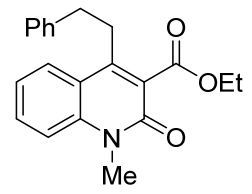

$2 \mathrm{f}$

$\left({ }^{13} \mathrm{C} \mathrm{NMR}, 101 \mathrm{MHz}, \mathrm{CDCl}_{3}\right)$

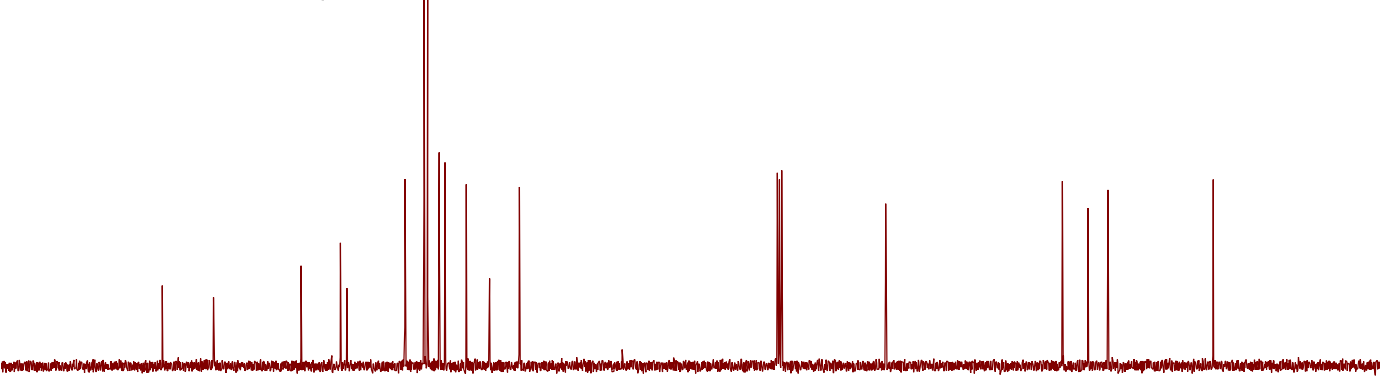

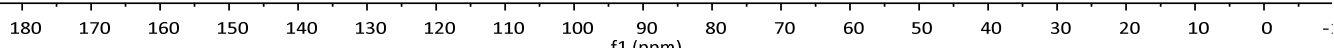

Ethyl 1-methyl-2-oxo-4-pentyl-1,2-dihydroquinoline-3-carboxylate (2g)

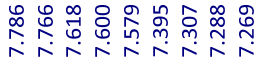

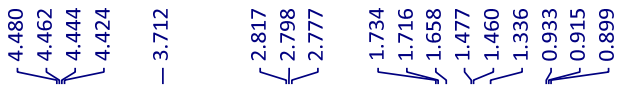<smiles>CCOC(=O)c1c(COC)c(=O)n(C)c2ccccc12</smiles>

$2 \mathrm{~g}$

( ${ }^{1} \mathrm{H} \mathrm{NMR}, 400 \mathrm{MHz}, \mathrm{CDCl}_{3}$ )

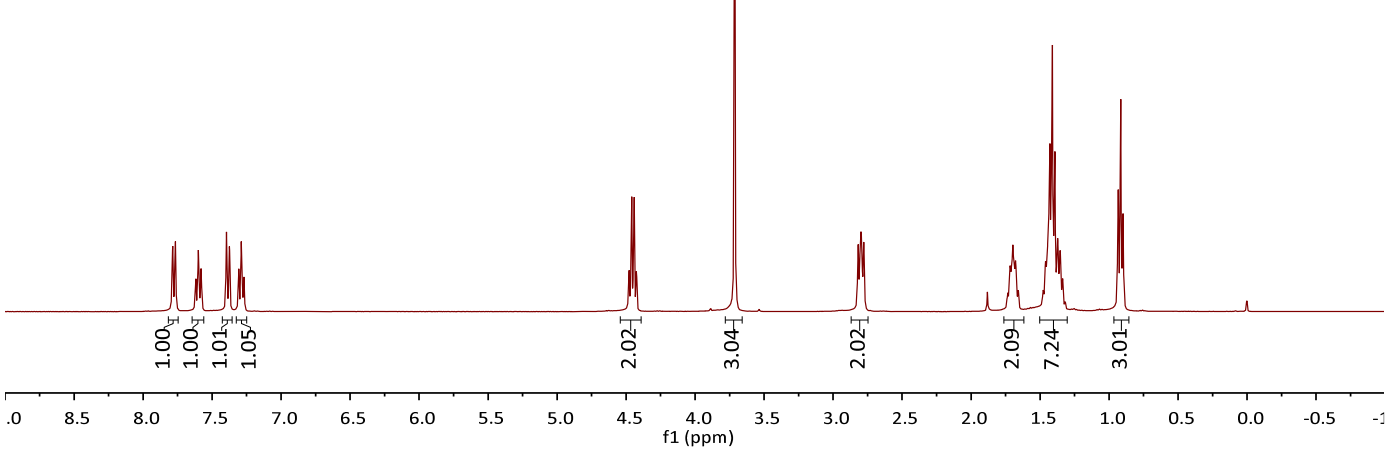




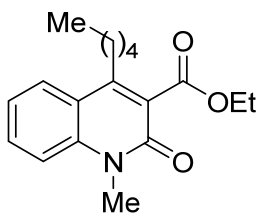

$\left({ }^{13} \mathrm{C}\right.$ NMR, $\left.101 \mathrm{MHz}, \mathrm{CDCl}_{3}\right)$
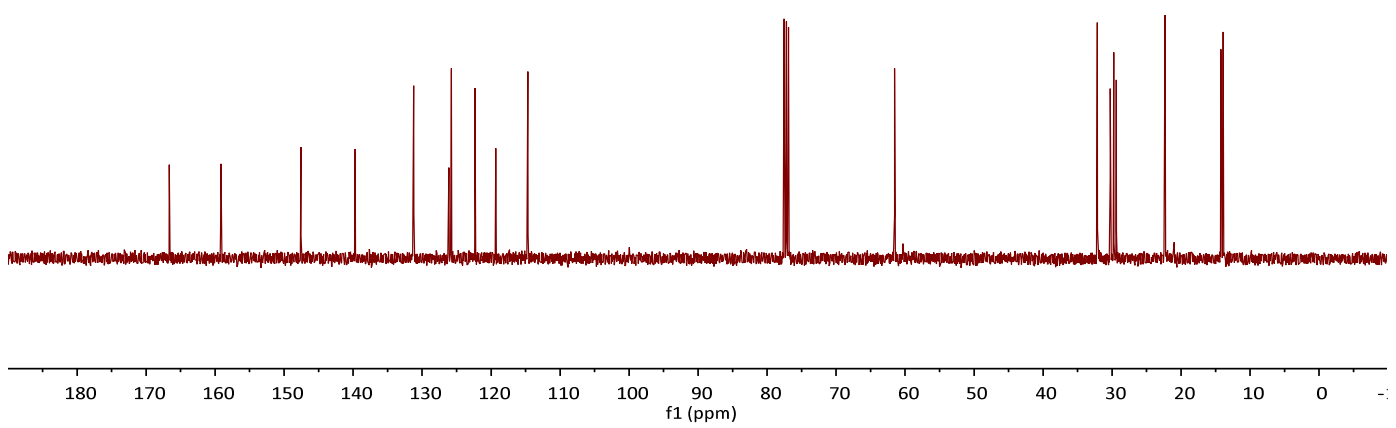

Ethyl 4-cyclopropyl-1-methyl-2-oxo-1,2-dihydroquinoline-3-carboxylate (2h)

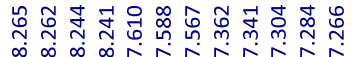

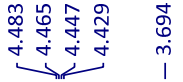

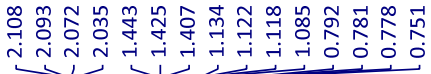<smiles>CCOC(=O)c1c(C2CC2)c2ccccc2n(C)c1=O</smiles>

2h

$\left({ }^{1} \mathrm{H} \mathrm{NMR}, 400 \mathrm{MHz}, \mathrm{CDCl}_{3}\right.$ )

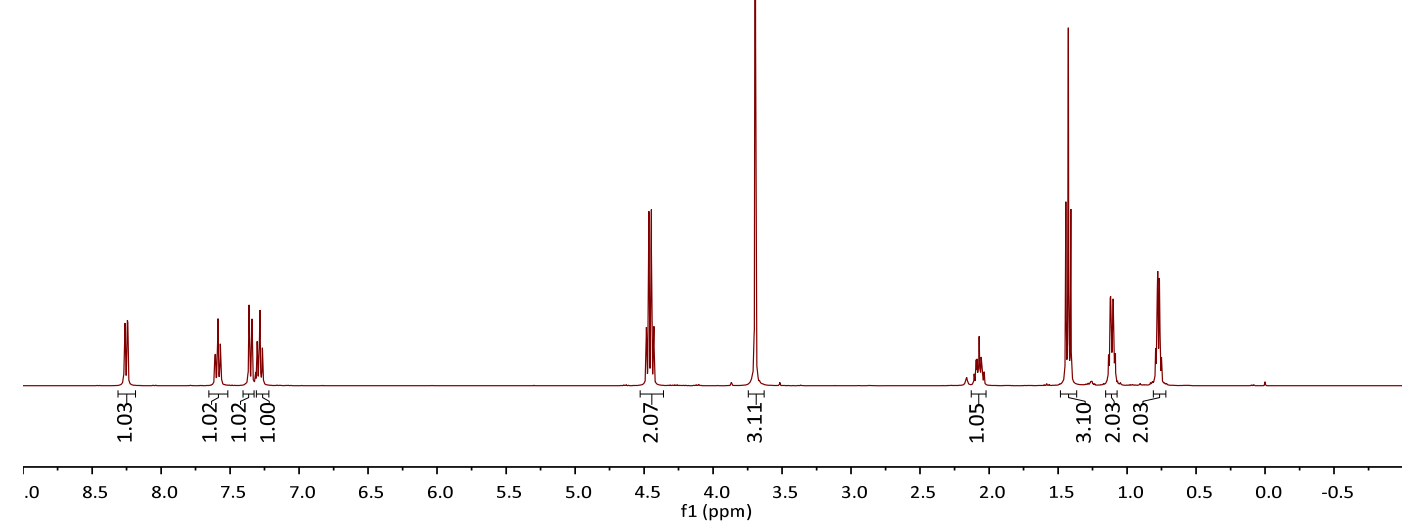


<smiles>CCOC(=O)c1c(C2CC2)c2ccccc2n(C)c1=O</smiles>

2h

$\left({ }^{13} \mathrm{C} \mathrm{NMR}, 101 \mathrm{MHz}, \mathrm{CDCl}_{3}\right)$

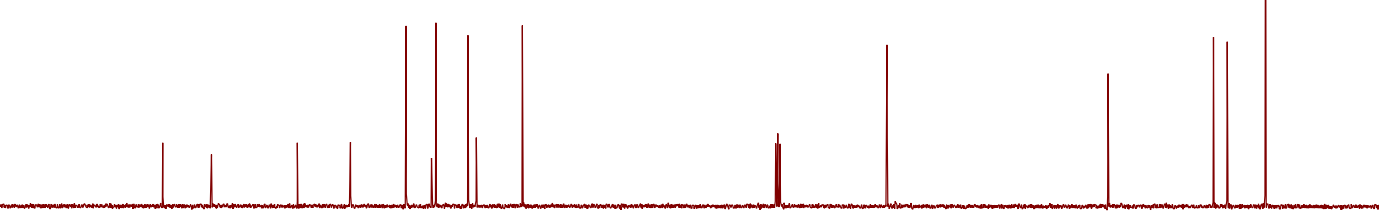

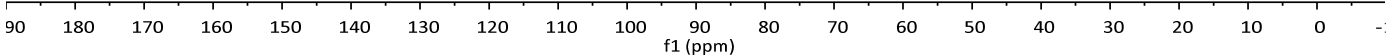

Ethyl 1-methyl-2-oxo-4-phenyl-1,2-dihydroquinoline-3-carboxylate (2i)<smiles>CCOC(=O)c1c(-c2ccccc2)c2ccccc2n(C)c1=O</smiles>

2i

$\left({ }^{1} \mathrm{H}\right.$ NMR, $\left.400 \mathrm{MHz}, \mathrm{CDCl}_{3}\right)$

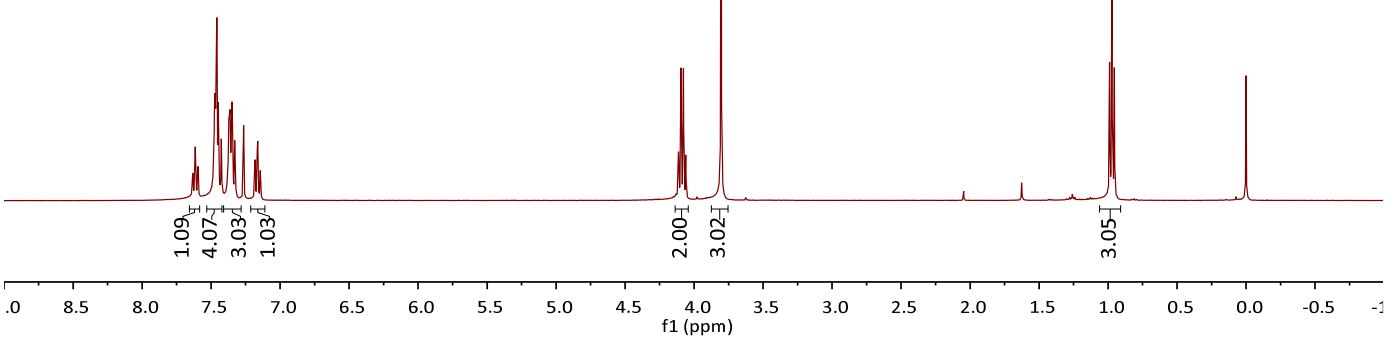


Ethyl 6-bromo-1,4-dimethyl-2-oxo-1,2-dihydroquinoline-3-carboxylate (2j)

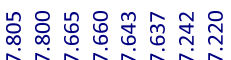

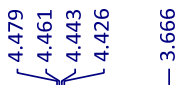

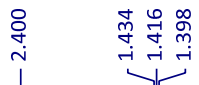<smiles>CCOC(=O)c1c([N+](=O)[O-])c2cc(Br)ccc2n([N+](=O)[O-])c1=O</smiles>

2j

$\left({ }^{1} \mathrm{H} \mathrm{NMR}, 400 \mathrm{MHz}, \mathrm{CDCl}_{3}\right)$
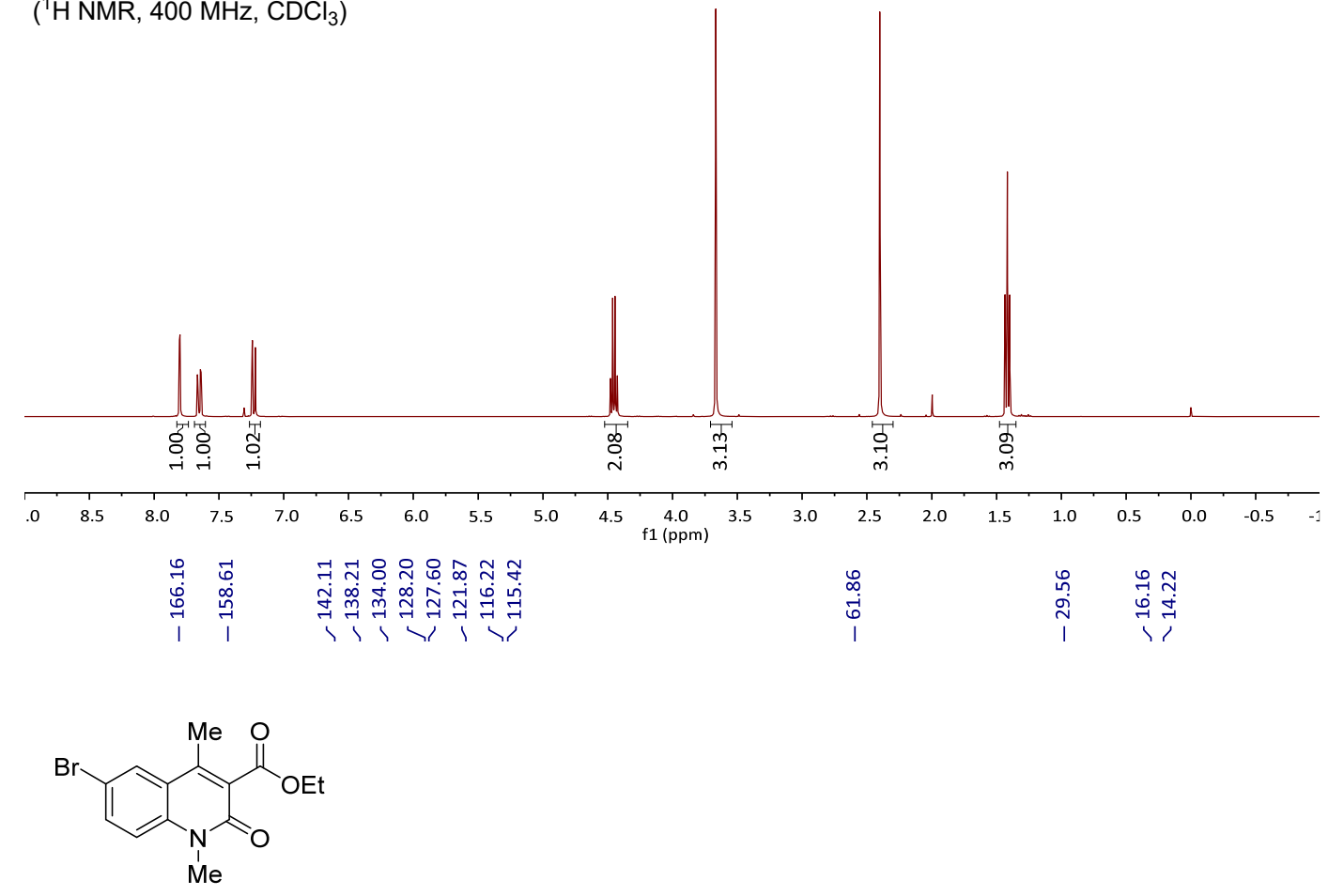

2j

$\left({ }^{13} \mathrm{C} \mathrm{NMR}, 101 \mathrm{MHz}, \mathrm{CDCl}_{3}\right)$

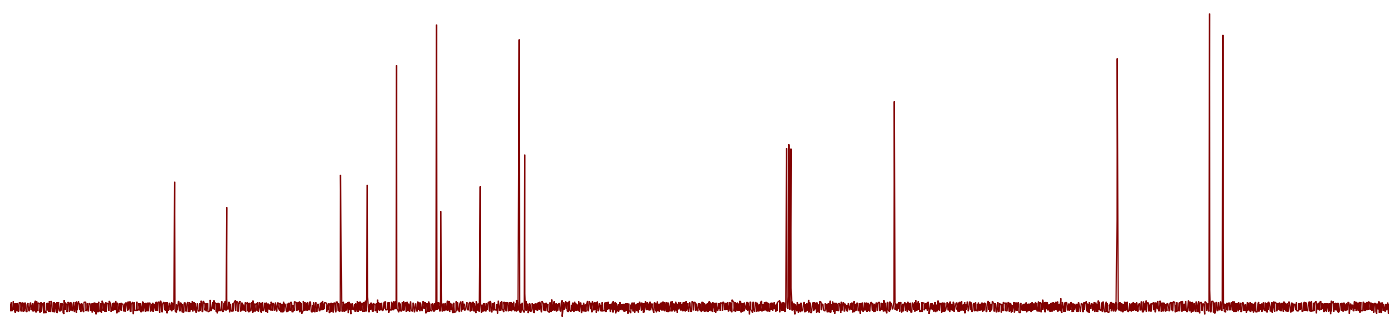

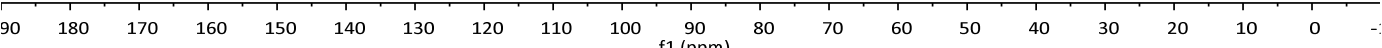


Ethyl 6-methoxy-1,4-dimethyl-2-oxo-1,2-dihydroquinoline-3-carboxylate (2k)

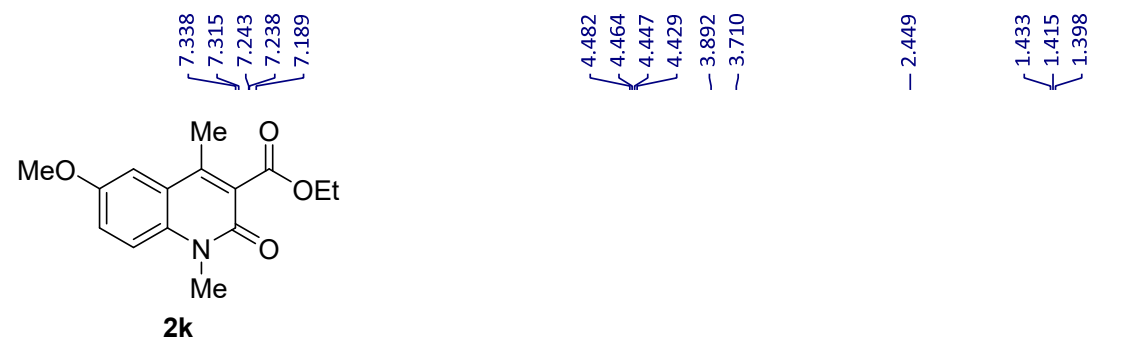

$\left({ }^{1} \mathrm{H}\right.$ NMR, $\left.400 \mathrm{MHz}, \mathrm{CDCl}_{3}\right)$
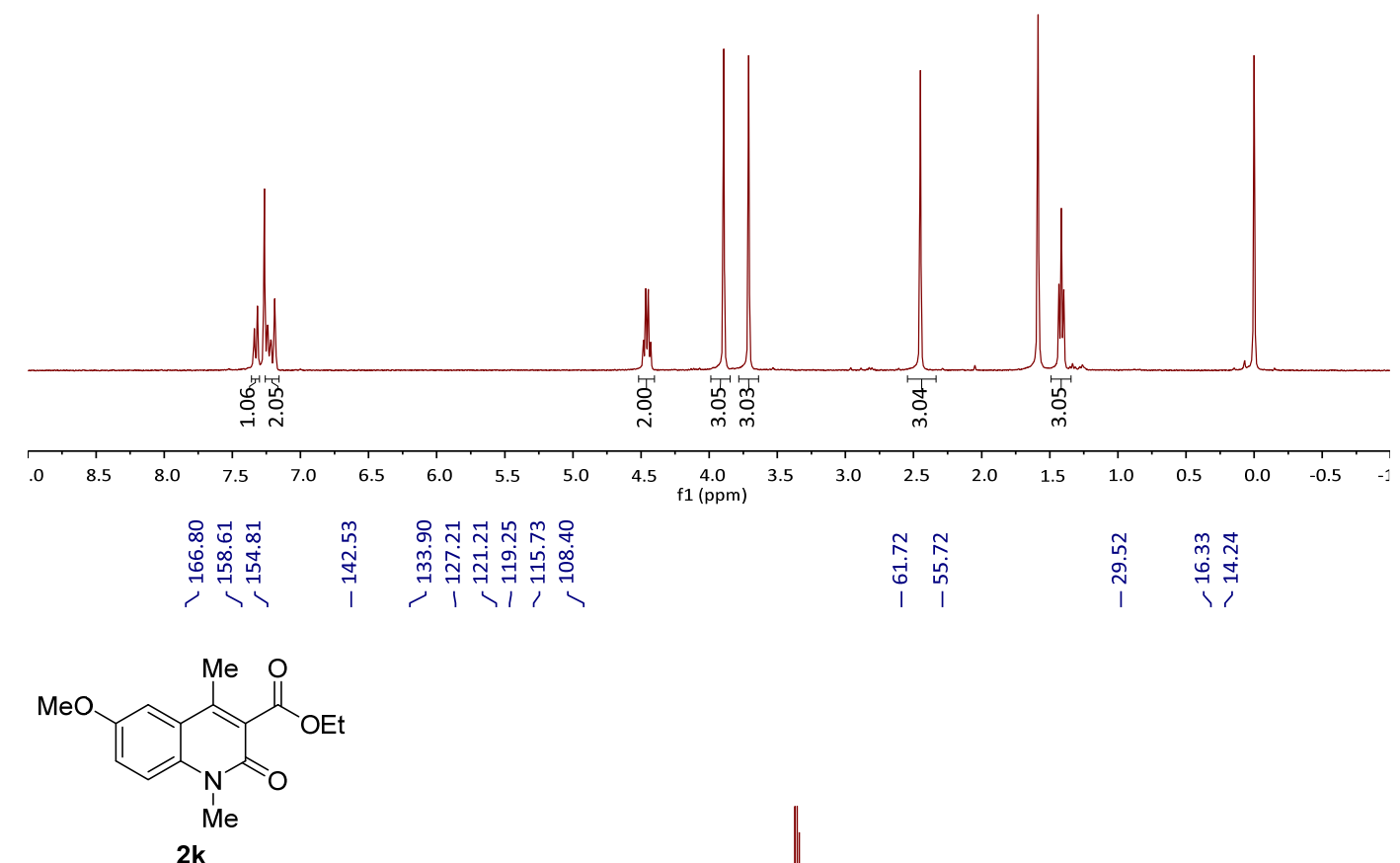

$\left({ }^{13} \mathrm{C} \mathrm{NMR}, 101 \mathrm{MHz}, \mathrm{CDCl}_{3}\right)$

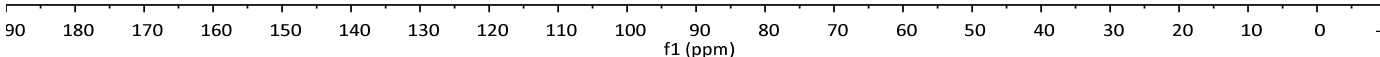


Ethyl 1-ethyl-4-methyl-2-oxo-1,2-dihydroquinoline-3-carboxylate (2l)

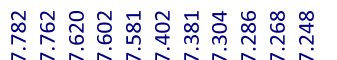

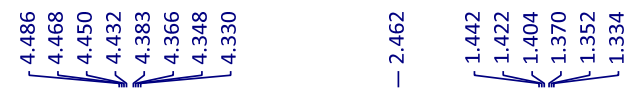<smiles>CCOC(=O)c1c(C)c2ccccc2n(CC)c1=O</smiles>

21

$\left({ }^{1} \mathrm{H}\right.$ NMR, $\left.400 \mathrm{MHz}, \mathrm{CDCl}_{3}\right)$

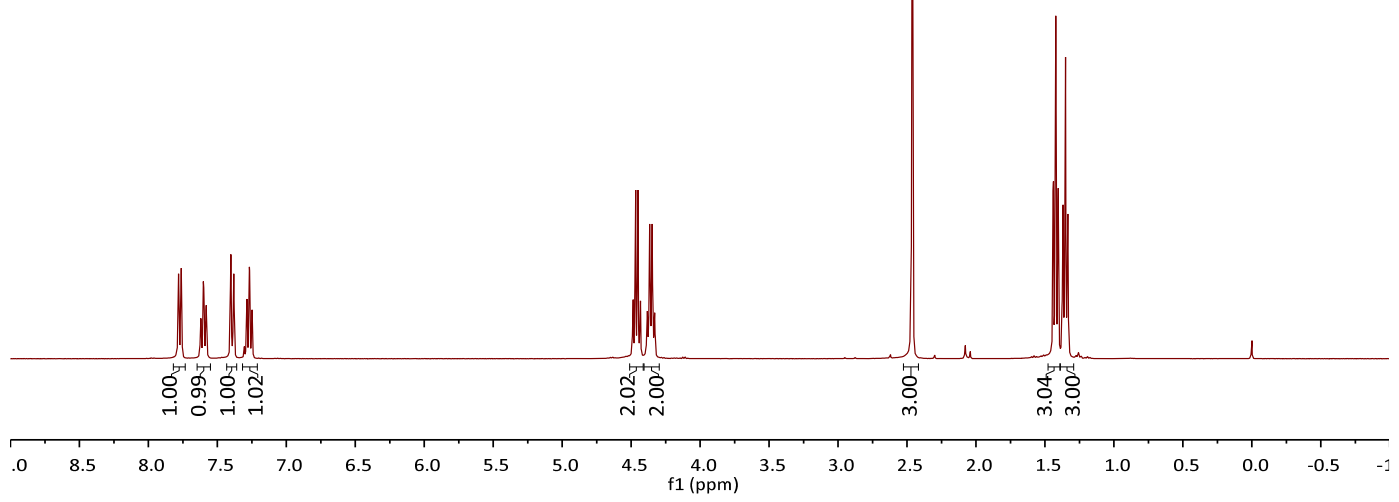

Ethyl 1-benzyl-4-methyl-2-oxo-1,2-dihydroquinoline-3-carboxylate (2m)

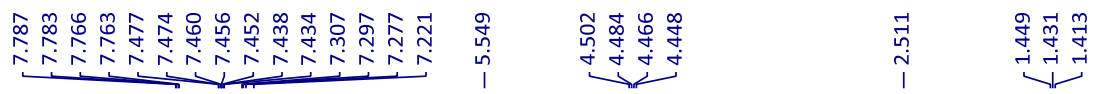<smiles>CCOC(=O)c1c(C)c2ccccc2n(Cc2ccccc2)c1=O</smiles>

2m

$\left({ }^{1} \mathrm{H} \mathrm{NMR}, 400 \mathrm{MHz}, \mathrm{CDCl}_{3}\right)$

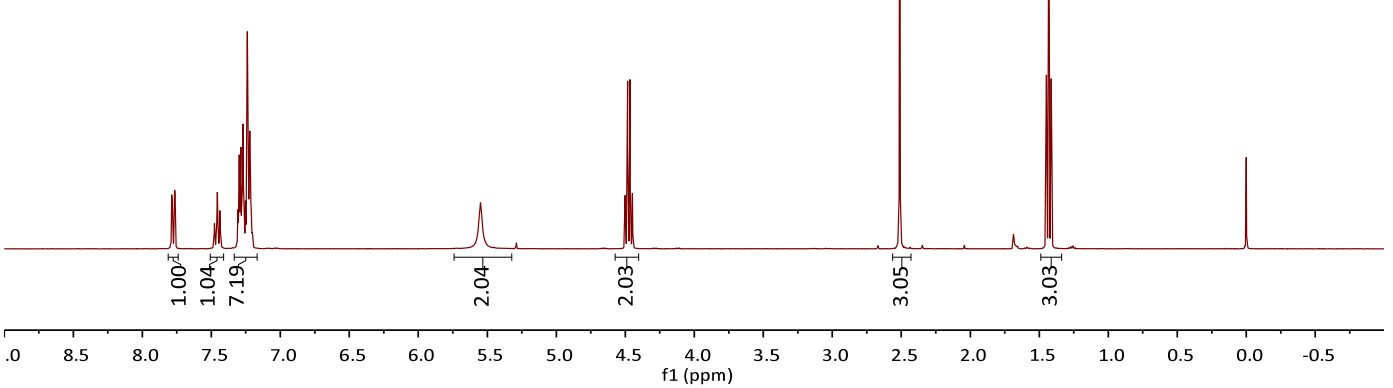




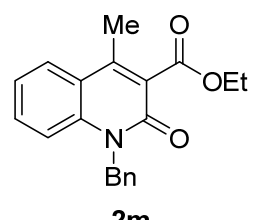

$\left({ }^{13} \mathrm{C} \mathrm{NMR}, 101 \mathrm{MHz}, \mathrm{CDCl}_{3}\right)$

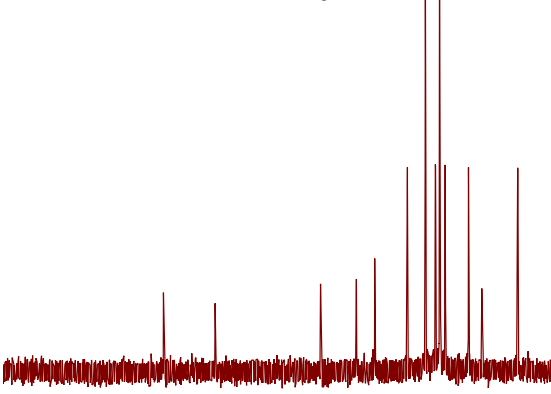

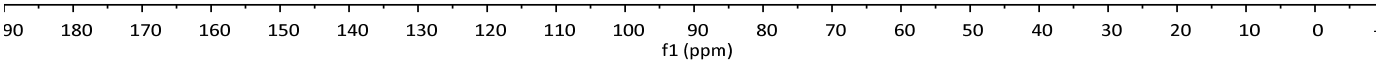

Ethyl 4-methyl-2-oxo-1-phenyl-1,2-dihydroquinoline-3-carboxylate (2n)

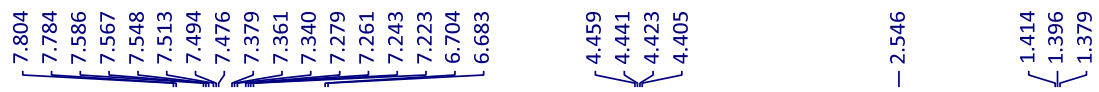<smiles>CCOC(=O)c1c(C)c2ccccc2n(-c2ccccc2)c1=O</smiles>

2n

$\left({ }^{1} \mathrm{HNMR}, 400 \mathrm{MHz}, \mathrm{CDCl}_{3}\right)$

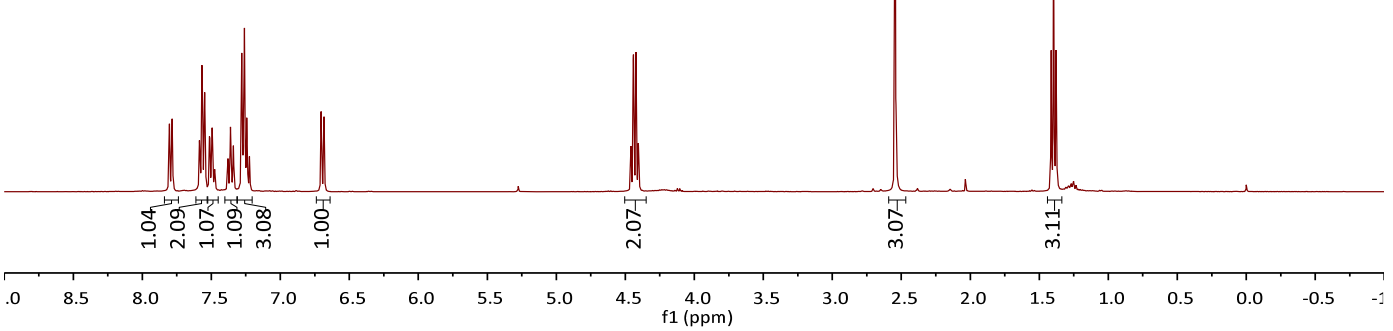




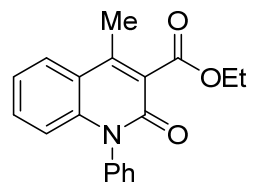

$\mathrm{Ph}$

2n

$\left({ }^{13} \mathrm{C} \mathrm{NMR}, 101 \mathrm{MHz}, \mathrm{CDCl}_{3}\right)$

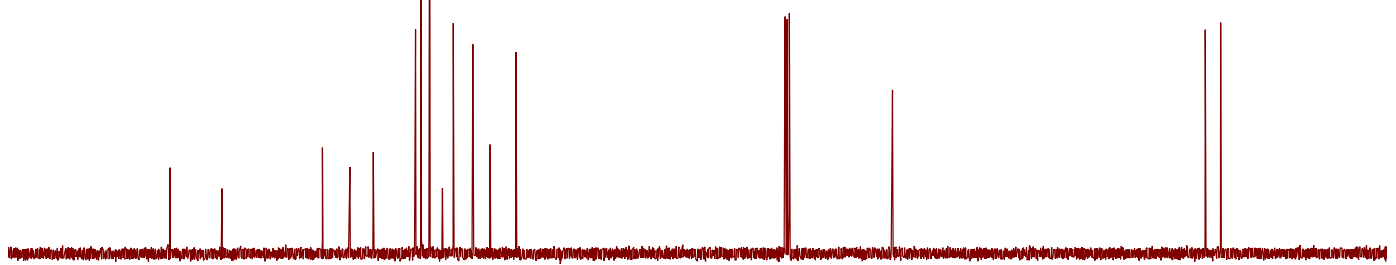

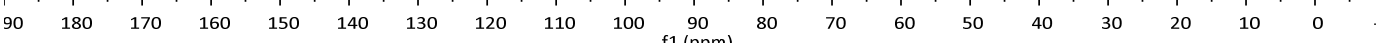

Ethyl 4-ethyl-2-oxo-1-phenyl-1,2-dihydroquinoline-3-carboxylate (20)

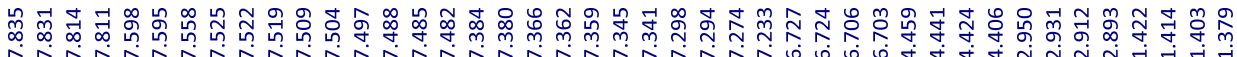<smiles>CCOC(=O)c1c(CC)c2ccccc2[nH]c1=O</smiles>

$\mathrm{Ph}$

20

$\left({ }^{1} \mathrm{H} \mathrm{NMR}, 400 \mathrm{MHz}, \mathrm{CDCl}_{3}\right)$

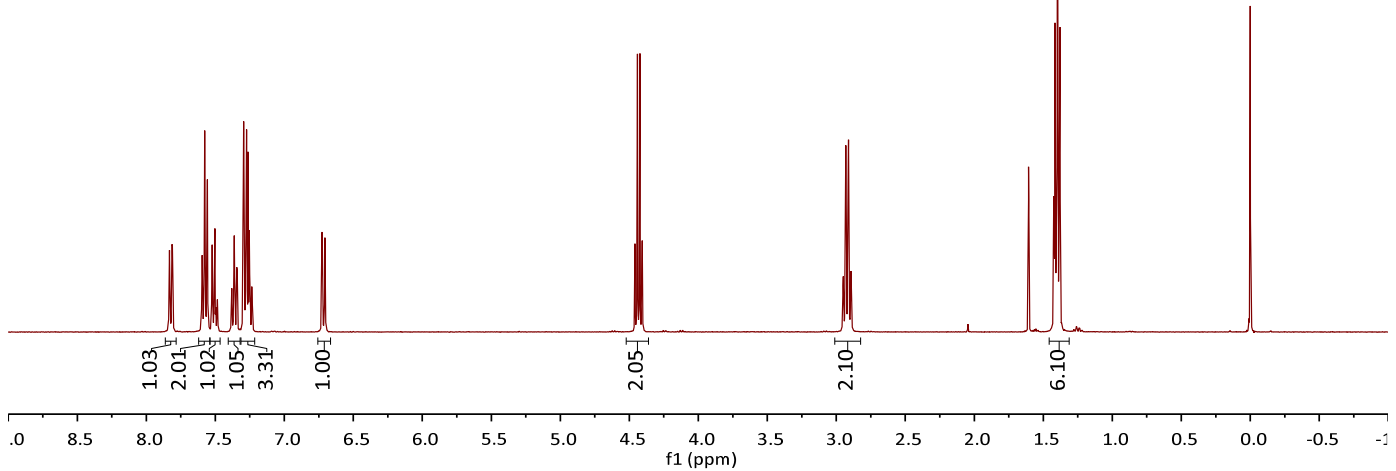




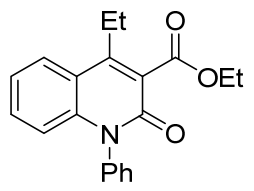

20

$\left({ }^{13} \mathrm{C} \mathrm{NMR}, 101 \mathrm{MHz}, \mathrm{CDCl}_{3}\right)$

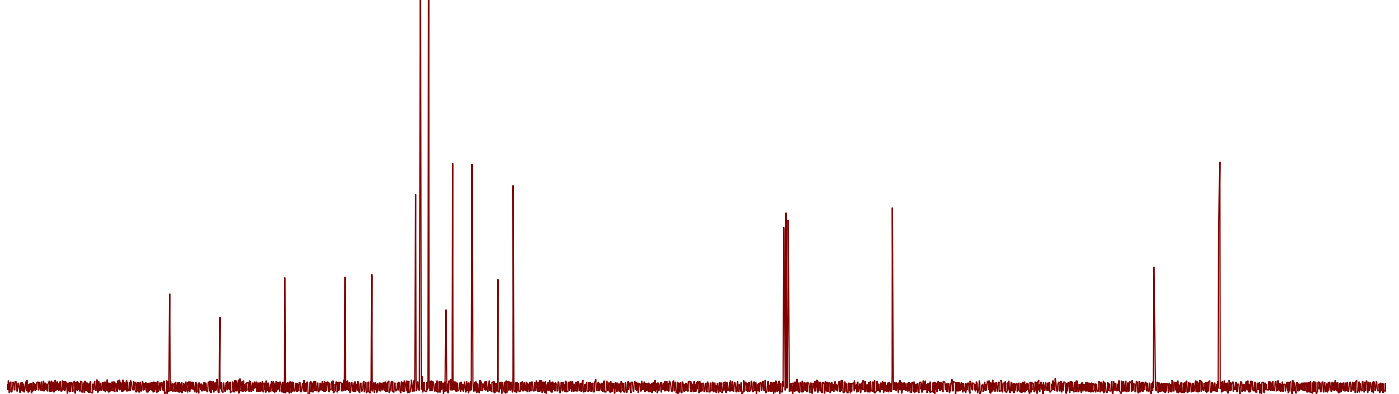

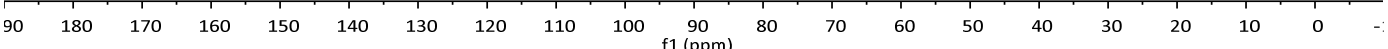

Ethyl 4-methyl-2-oxo-2H-chromene-3-carboxylate (4a)

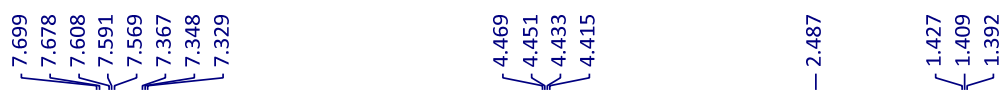<smiles>CCOC(=O)c1c(C)c2ccccc2oc1=O</smiles>

4a

$\left({ }^{1} \mathrm{H}\right.$ NMR, $\left.400 \mathrm{MHz}, \mathrm{CDCl}_{3}\right)$

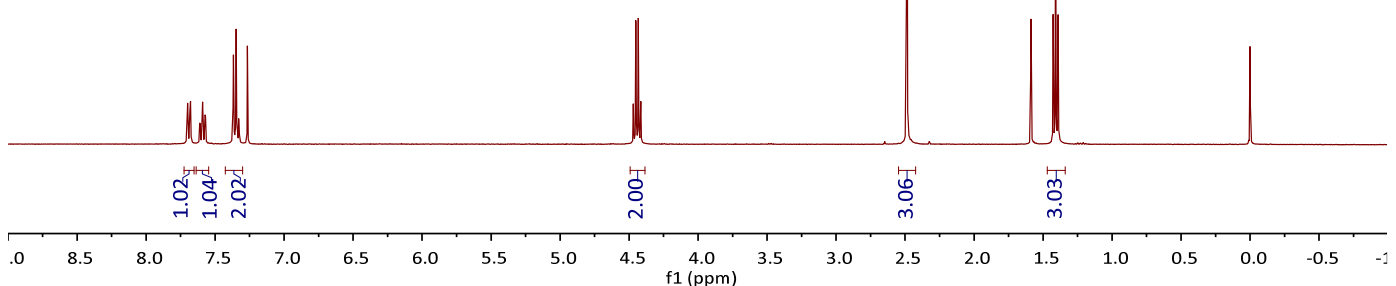


Ethyl 4-ethyl-2-oxo-2H-chromene-3-carboxylate (4b)

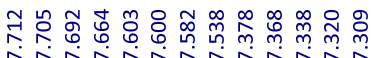

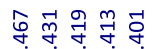

نـ<smiles>CCOC(=O)c1c(CC)c2ccccc2oc1=O</smiles>

4b

( ${ }^{1} \mathrm{H} \mathrm{NMR}, 400 \mathrm{MHz}, \mathrm{CDCl}_{3}$ )

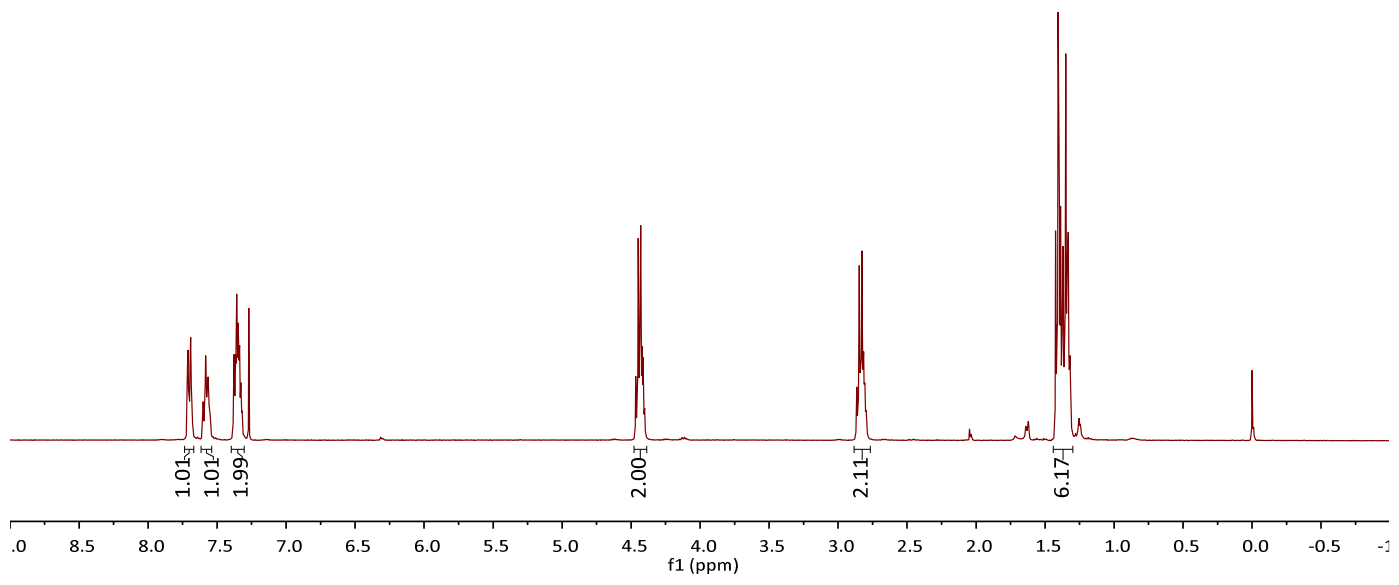

Ethyl 2-oxo-4-propyl-2H-chromene-3-carboxylate (4c)

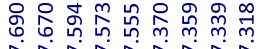

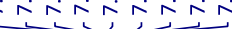

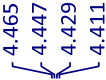

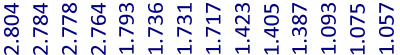<smiles>CCOC(=O)c1c(C(C)C)c2ccccc2oc1=O</smiles>

$4 \mathrm{c}$

$\left({ }^{1} \mathrm{H} \mathrm{NMR}, 400 \mathrm{MHz}, \mathrm{CDCl}_{3}\right)$

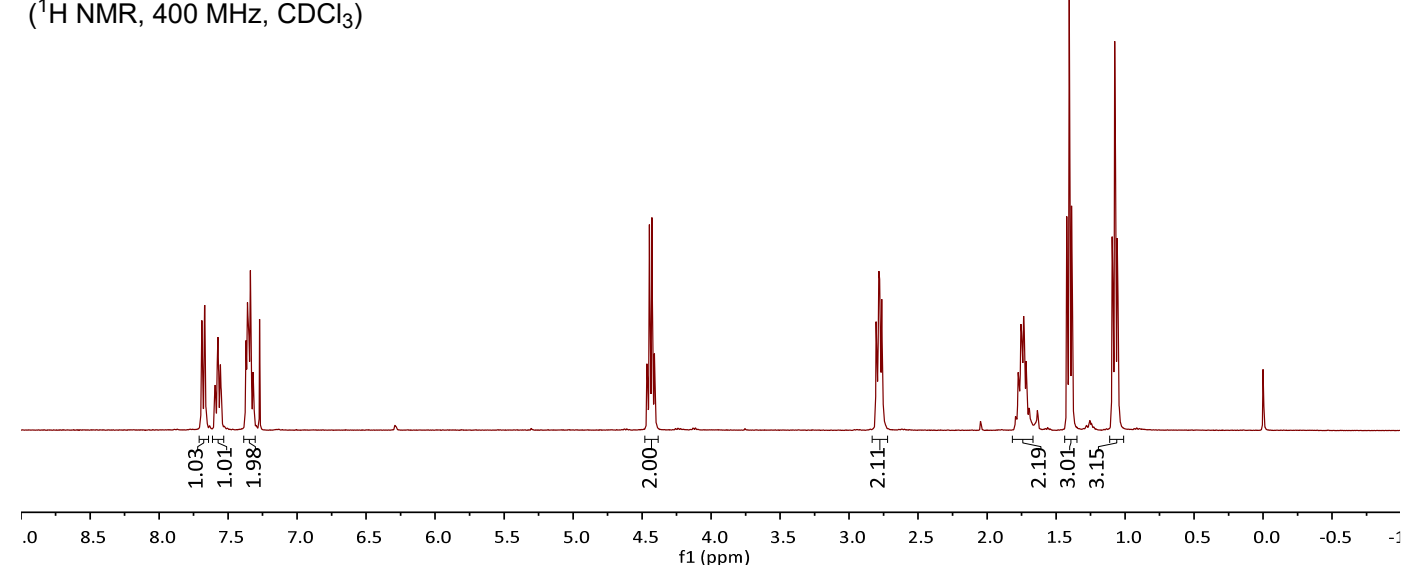


Ethyl-4-isobutyl-2-oxo-2H-chromene-3-carboxylate (4d)

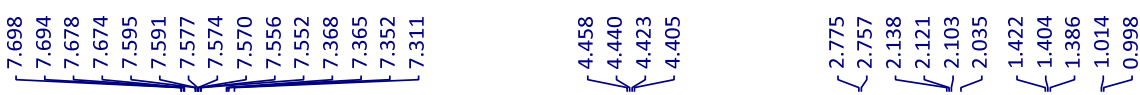<smiles>CCOC(=O)c1c(CC(C)C)c2ccccc2oc1=O</smiles>

4d

( ${ }^{1} \mathrm{H}$ NMR, $400 \mathrm{MHz}, \mathrm{CDCl}_{3}$ )

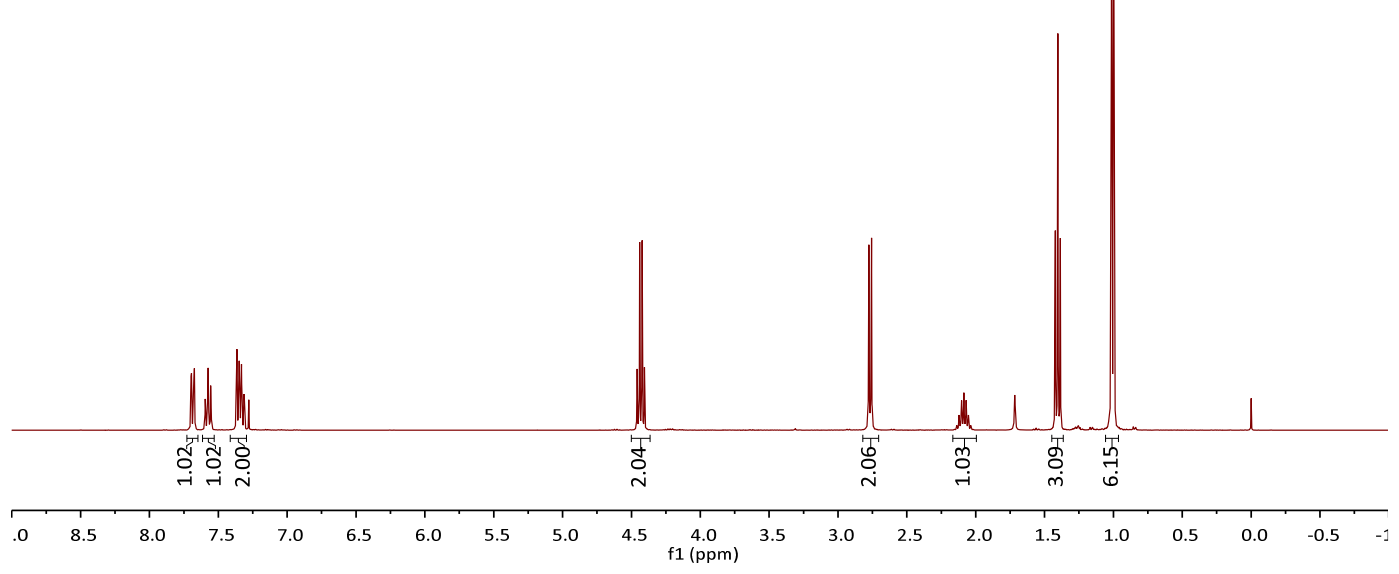<smiles>CCOC(=O)c1c(CC(C)C)c2ccccc2oc1=O</smiles>

4d

$\left({ }^{13} \mathrm{C} \mathrm{NMR}, 101 \mathrm{MHz}, \mathrm{CDCl}_{3}\right)$

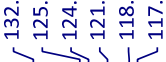

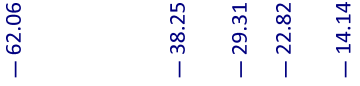

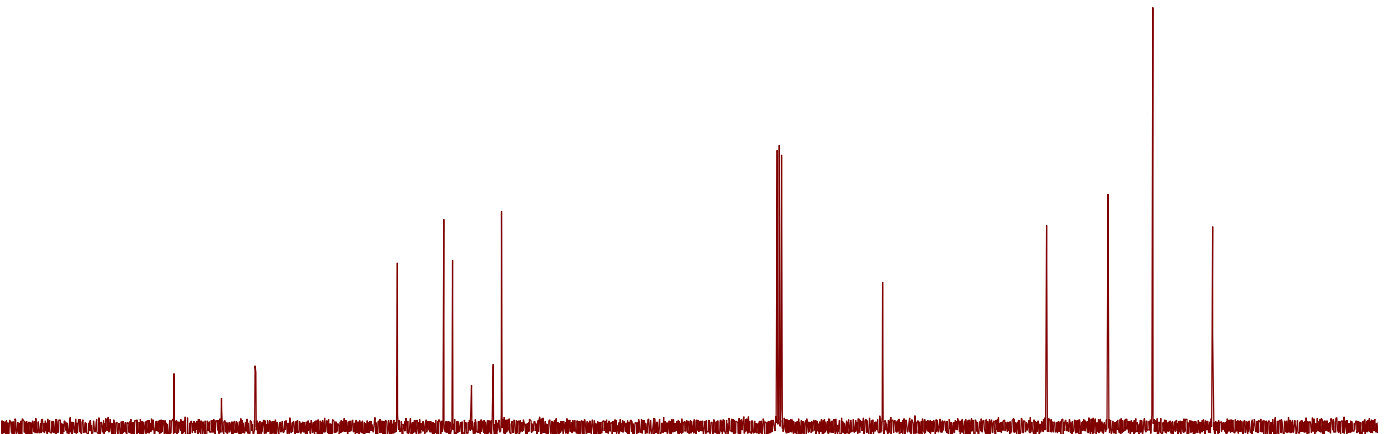

\begin{tabular}{llllllllllllllllllll}
\hline 0 & 180 & 170 & 160 & 150 & 140 & 130 & 120 & 110 & 100 & 90 & 80 & 70 & 60 & 50 & 40 & 30 & 20 & 10 & 0
\end{tabular} 
Ethyl-4-isopropyl-2-oxo-2H-chromene-3-carboxylate (4e)

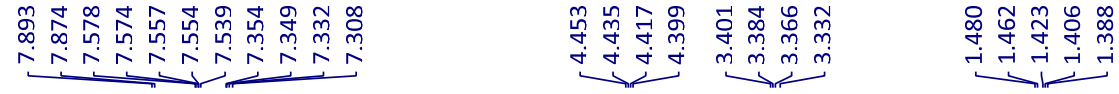<smiles>CCOC(=O)c1c(C(C)C)c2ccccc2oc1=O</smiles>

$4 e$

('H NMR, $400 \mathrm{MHz}, \mathrm{CDCl}_{3}$ )
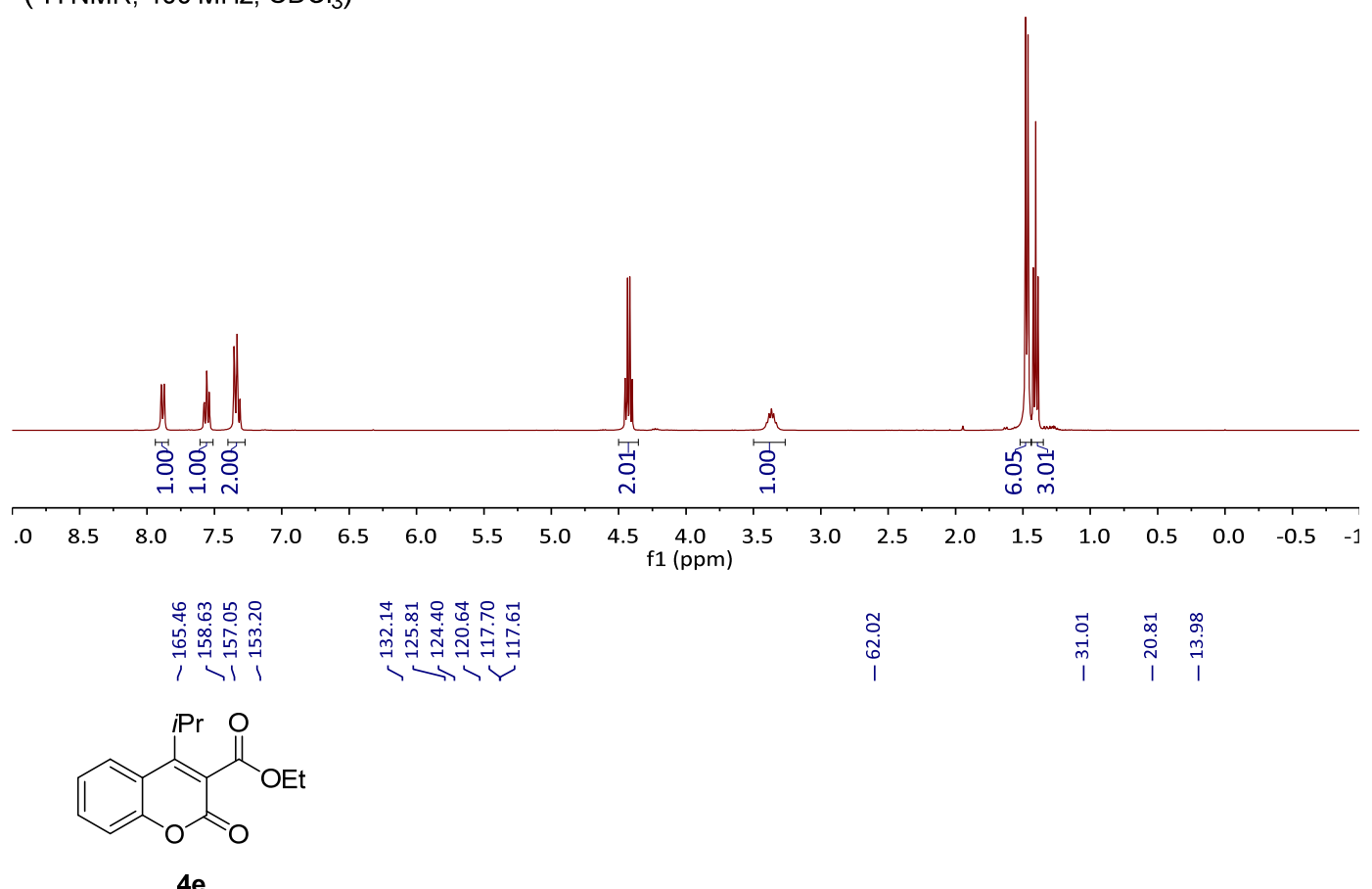

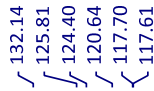

$\begin{array}{llll}\tilde{0} & \overrightarrow{0} & \overrightarrow{0} & \infty \\ \dot{0} & 1 & \stackrel{\sim}{0} & \stackrel{m}{m} \\ 1 & 1 & 1 & 1\end{array}$

$\left({ }^{13} \mathrm{C}\right.$ NMR, $\left.101 \mathrm{MHz}, \mathrm{CDCl}_{3}\right)$

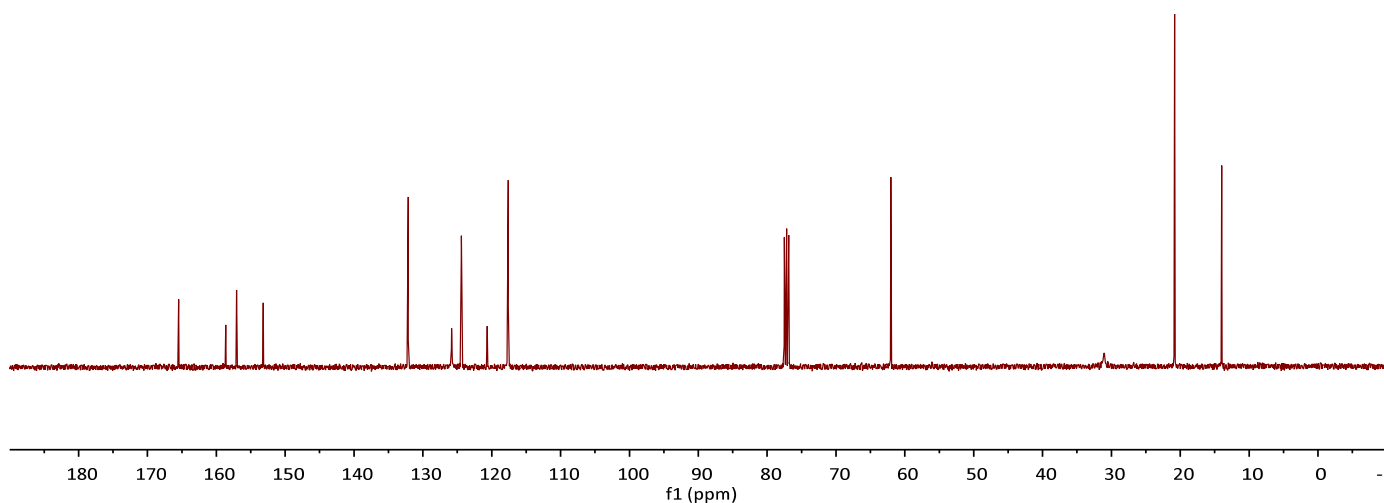


Ethyl-4-cyclohexyl-2-oxo-2H-chromene-3-carboxylate (4f)

\begin{tabular}{|c|c|c|c|}
\hline 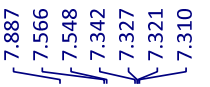 & 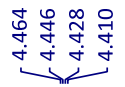 & ֻे & 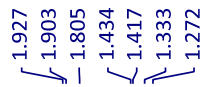 \\
\hline
\end{tabular}

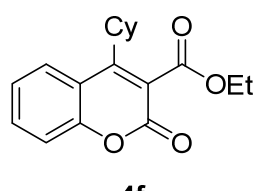

$4 f$

$\left({ }^{1} \mathrm{H}\right.$ NMR, $\left.400 \mathrm{MHz}, \mathrm{CDCl}_{3}\right)$

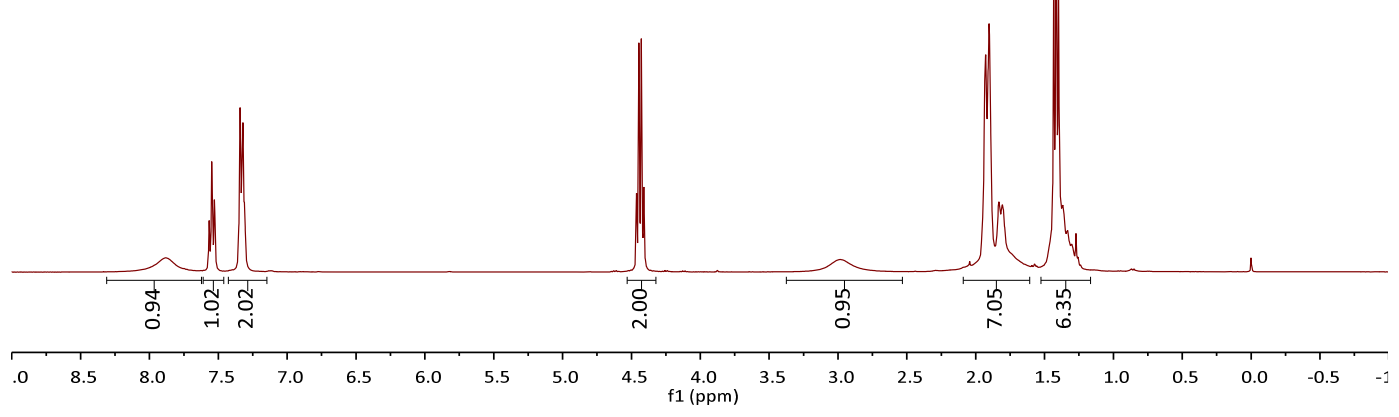

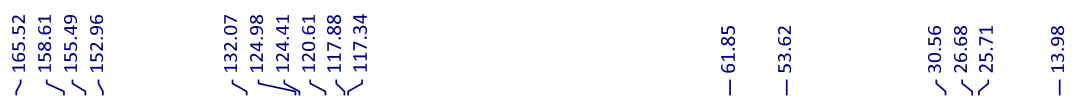

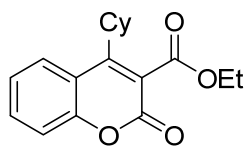

$4 f$

$\left({ }^{13} \mathrm{C} \mathrm{NMR}, 101 \mathrm{MHz}, \mathrm{CDCl}_{3}\right)$

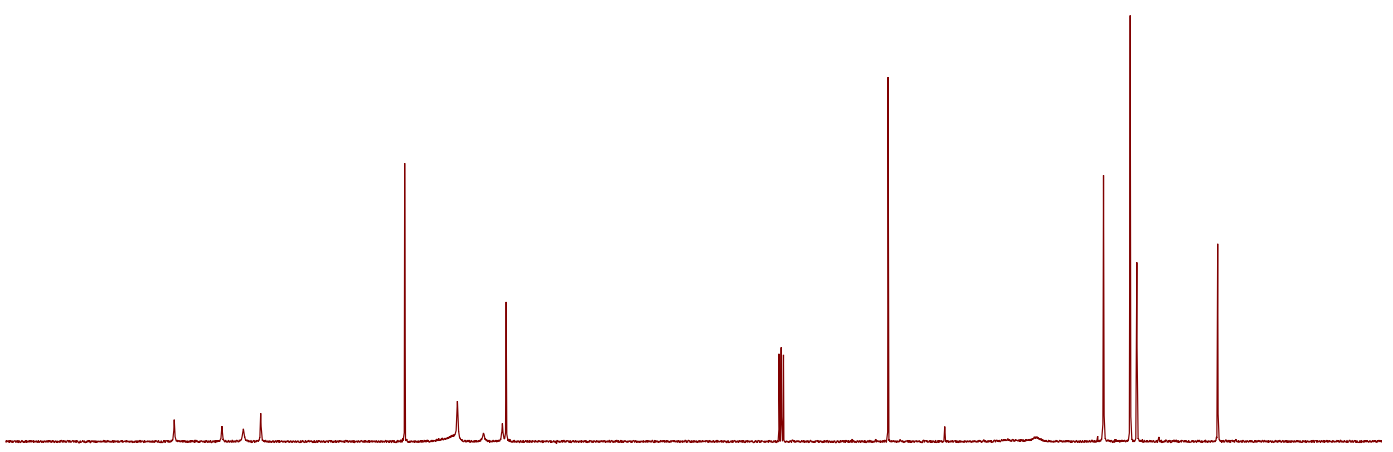

$\begin{array}{llllllllllllllllllll}90 & 180 & 170 & 160 & 150 & 140 & 130 & 120 & 110 & 100 & 90 & 80 & 70 & 60 & 50 & 40 & 30 & 20 & 10 & 0\end{array}$ 
Ethyl-4-benzyl-2-oxo-2H-chromene-3-carboxylate (4g)

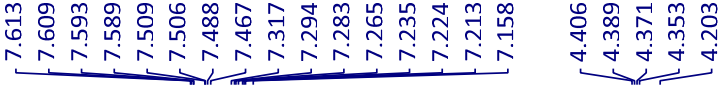<smiles>CCOC(=O)c1c(Cc2ccccc2)c2ccccc2oc1=O</smiles>

4g

( ${ }^{1} \mathrm{H}$ NMR, $400 \mathrm{MHz}_{2}, \mathrm{CDCl}_{3}$ )

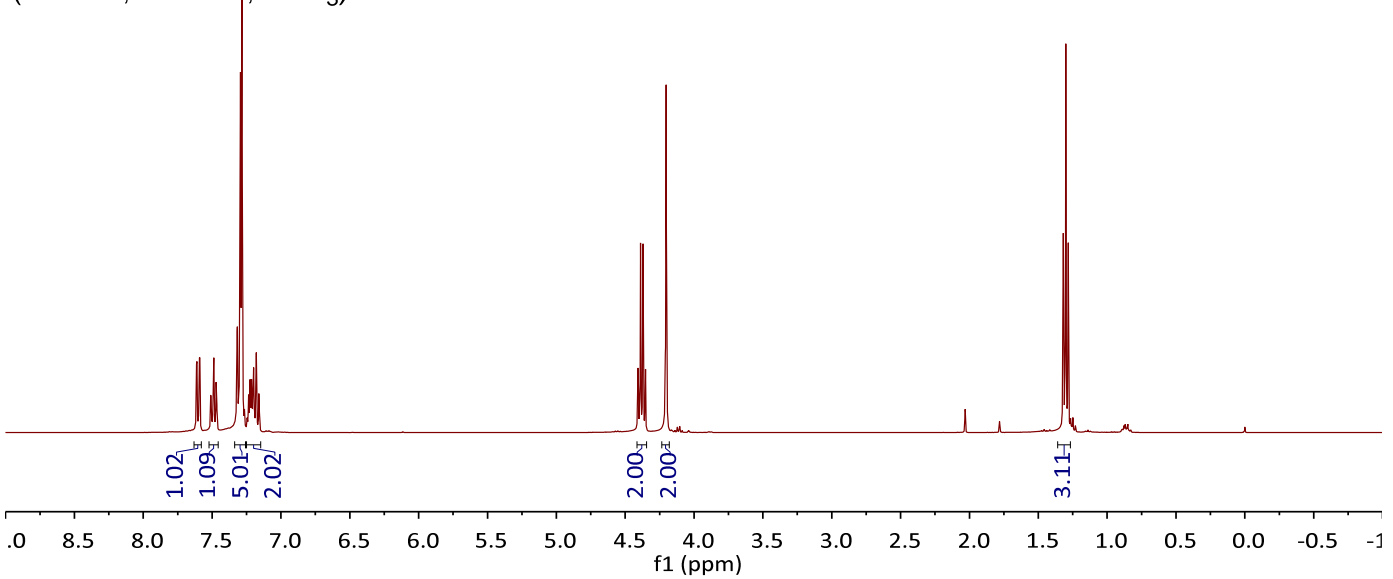

ำฺำ

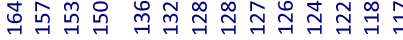

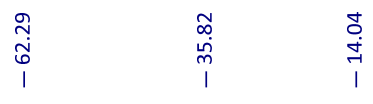<smiles>CCOC(=O)c1c(Cc2ccccc2)c2ccccc2oc1=O</smiles>

$4 \mathrm{~g}$

$\left({ }^{13} \mathrm{C} \mathrm{NMR}, 101 \mathrm{MHz}, \mathrm{CDCl}_{3}\right)$

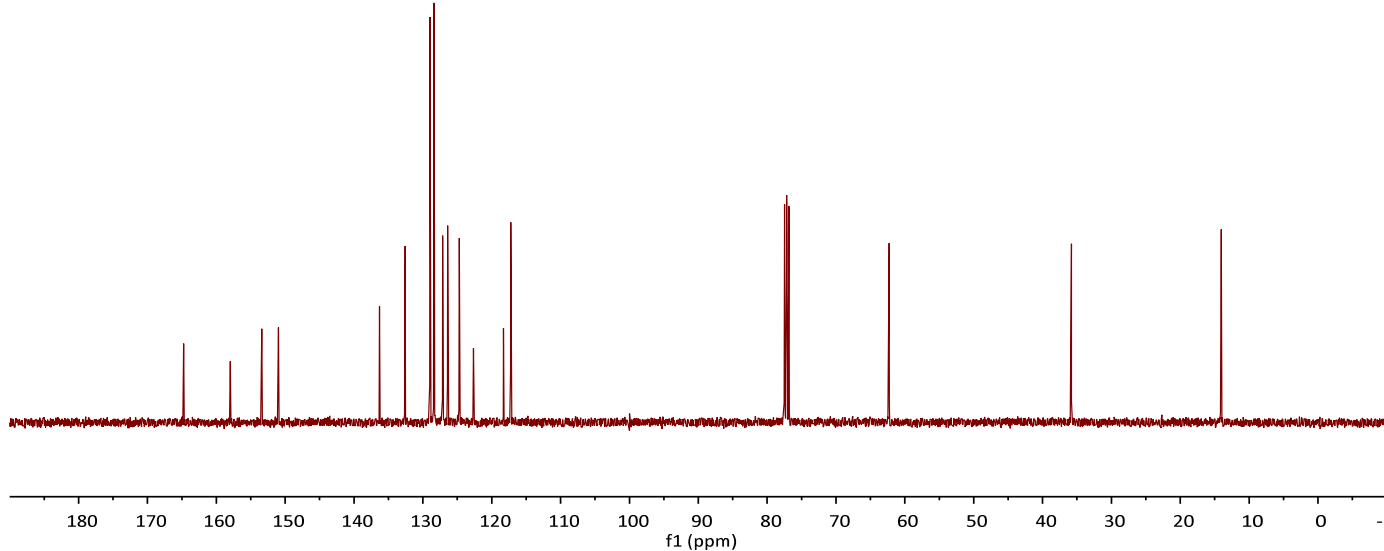


Ethyl-2-oxo-4-phenethyl-2H-chromene-3-carboxylate (4h)

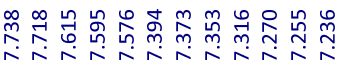

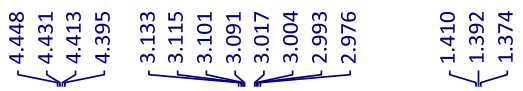<smiles>CCOC(=O)c1c(CCc2ccccc2)c2ccccc2oc1=O</smiles>

4h

$\left({ }^{1} \mathrm{H} \mathrm{NMR}, 400 \mathrm{MHz}, \mathrm{CDCl}_{3}\right)$

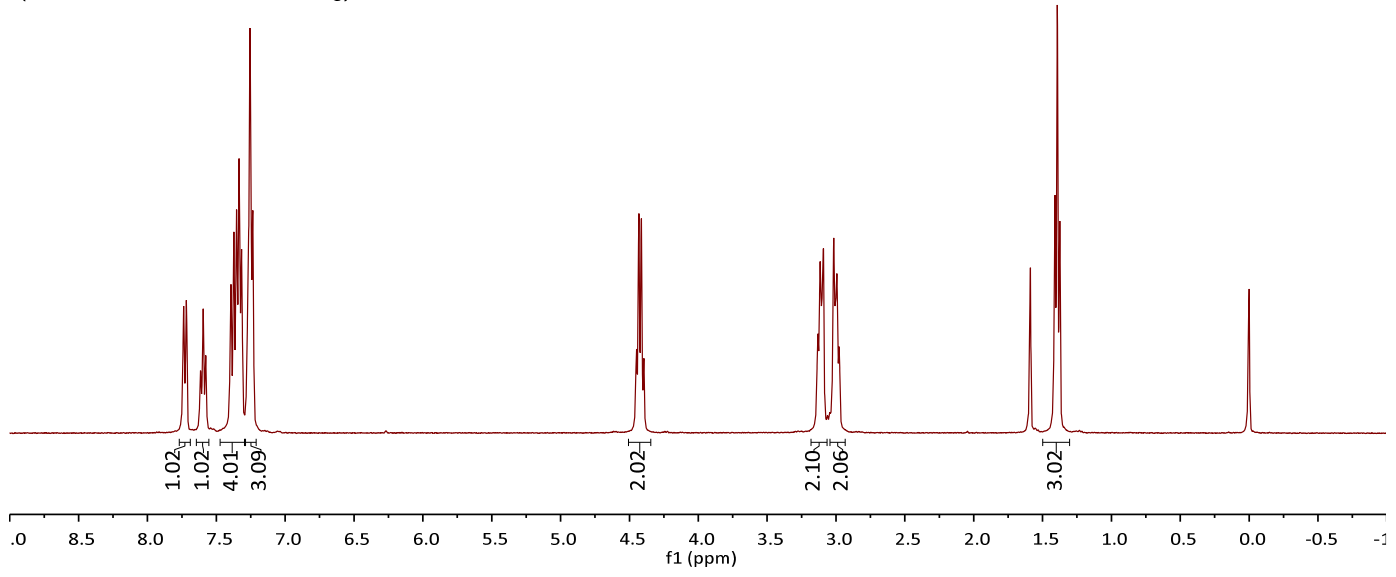

ำษ

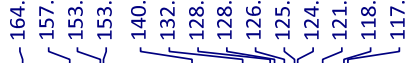

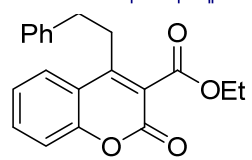

4h

$\left({ }^{13} \mathrm{C} \mathrm{NMR}, 101 \mathrm{MHz}, \mathrm{CDCl}_{3}\right)$

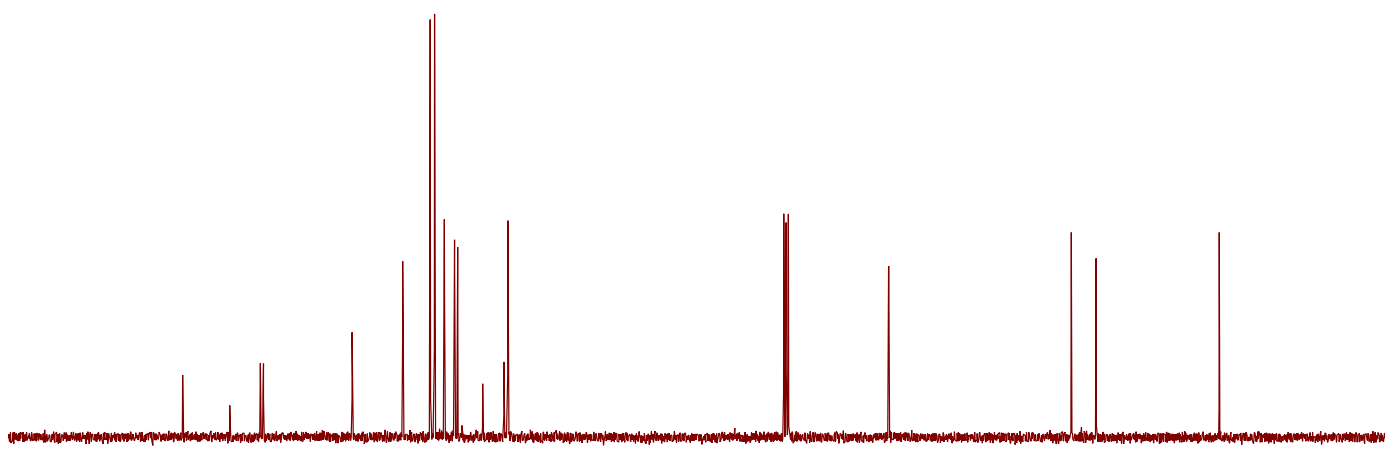

$\begin{array}{llllllllllllllllllll}90 & 180 & 170 & 160 & 150 & 140 & 130 & 120 & 110 & 100 & 90 & 80 & 70 & 60 & 50 & 40 & 30 & 20 & 10 & 0\end{array}$ 
Ethyl-4-(4-ethoxy-4-oxobutyl)-2-oxo-2H-chromene-3-carboxylate (4i)

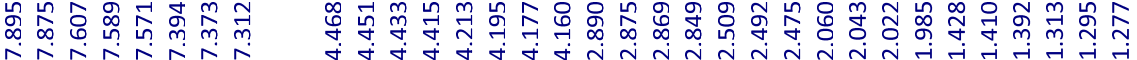

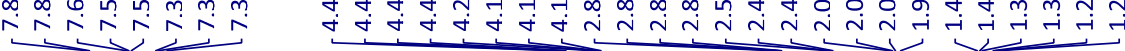

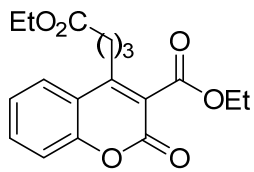

$4 \mathbf{i}$

( ${ }^{1} \mathrm{H} \mathrm{NMR}, 400 \mathrm{MHz}, \mathrm{CDCl}_{3}$ )

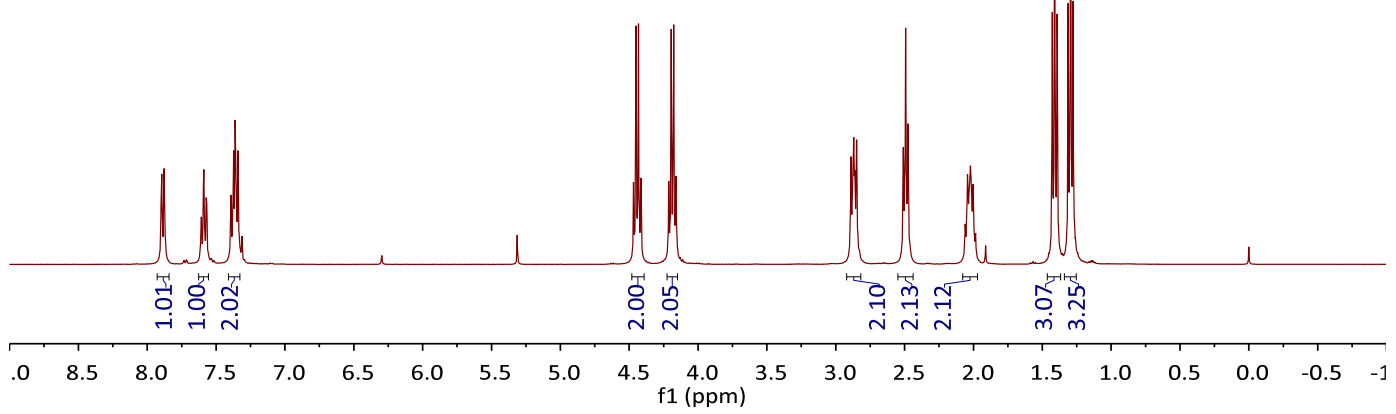

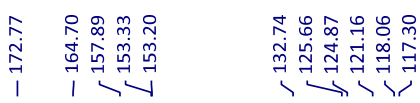
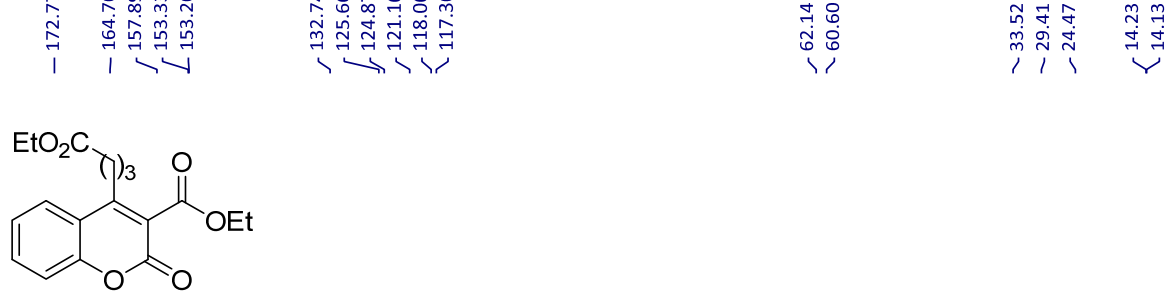

$4 \mathbf{i}$

$\left({ }^{13} \mathrm{C} \mathrm{NMR}, 101 \mathrm{MHz}, \mathrm{CDCl}_{3}\right)$

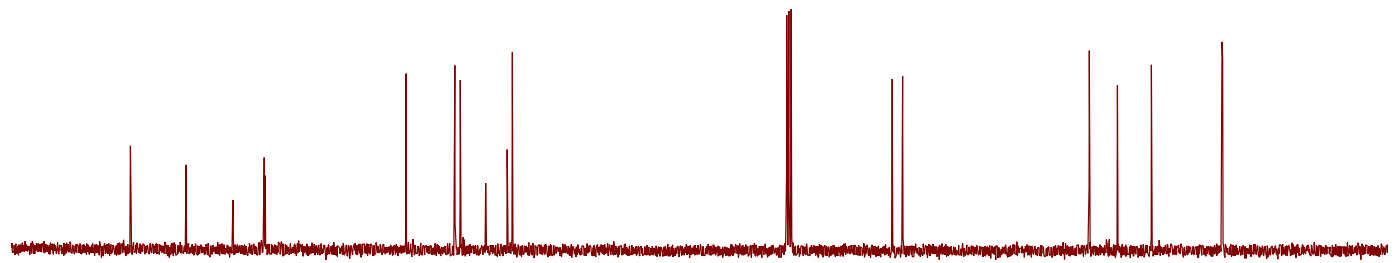

$\begin{array}{llllllllllllllllllll}90 & 180 & 170 & 160 & 150 & 140 & 130 & 120 & 110 & 100 & 90 & 80 & 70 & 60 & 50 & 40 & 30 & 20 & 10 & 0\end{array}$ 
Ethyl-4-(2-((tert-butoxycarbonyl)(methyl)amino)ethyl)-2-oxo-2H-chromene-3-carboxylate (4j)

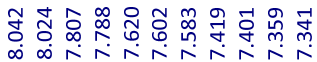

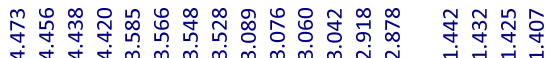

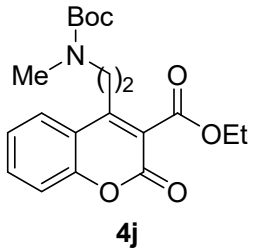

$\left({ }^{1} \mathrm{H} \mathrm{NMR}, 400 \mathrm{MHz}, \mathrm{CDCl}_{3}\right)$
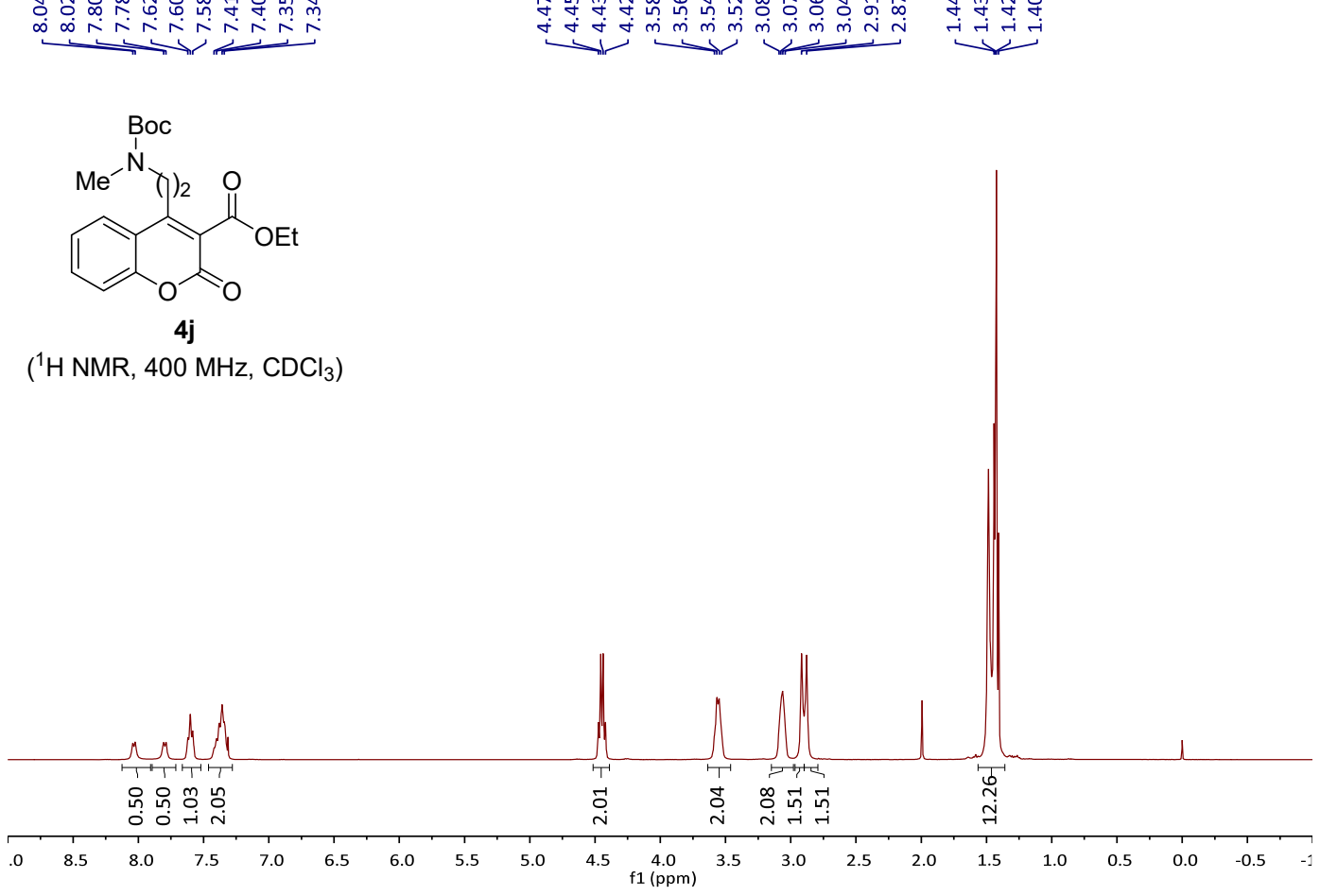

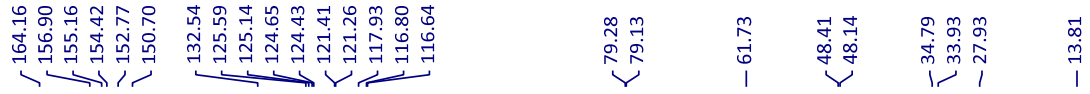

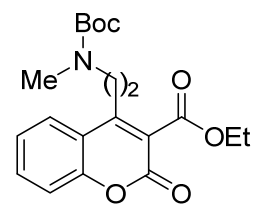

4j

$\left({ }^{13} \mathrm{C} \mathrm{NMR}, 101 \mathrm{MHz}, \mathrm{CDCl}_{3}\right)$

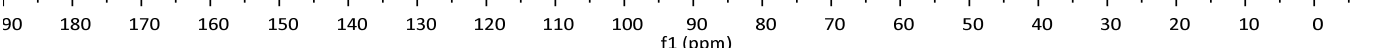


Ethyl-7-methoxy-2-oxo-4-pentyl-2H-chromene-3-carboxylate (4k)

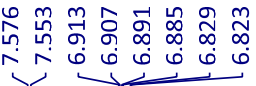

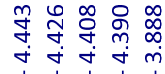

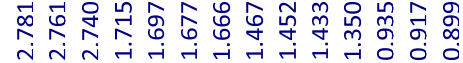

$\underbrace{4}$

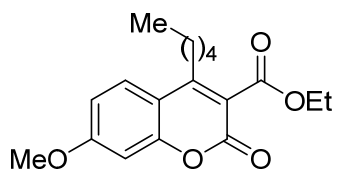

4k

$\left({ }^{1} \mathrm{HNMR}, 400 \mathrm{MHz}, \mathrm{CDCl}_{3}\right)$

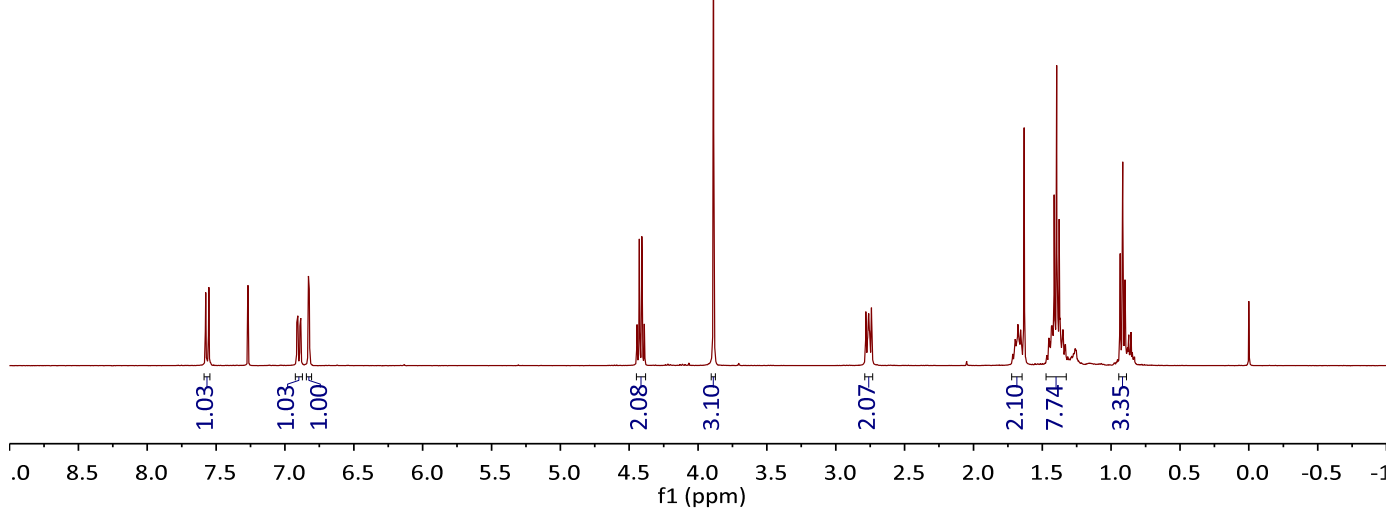

\begin{tabular}{|c|c|c|c|}
\hline 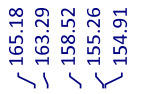 & 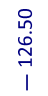 & 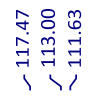 & $\begin{array}{l}\text { ô } \\
\stackrel{\circ}{0} \\
\stackrel{1}{1}\end{array}$ \\
\hline
\end{tabular}

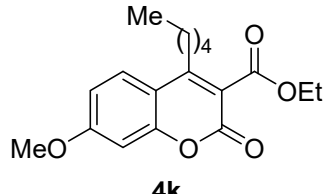

4k

$\left({ }^{13} \mathrm{C} \mathrm{NMR}, 101 \mathrm{MHz}, \mathrm{CDCl}_{3}\right)$

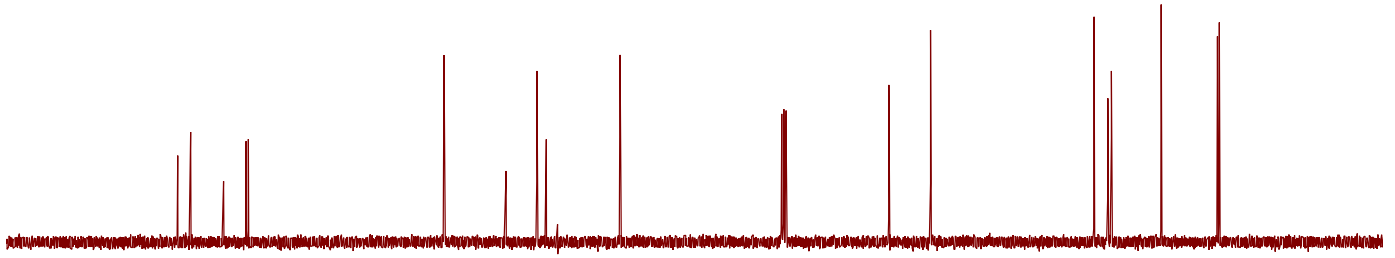

$\begin{array}{lllllllllllllllllllllllllll}90 & 180 & 170 & 160 & 150 & 140 & 130 & 120 & 110 & 100 & 90 & 80 & 70 & 60 & 50 & 40 & 30 & 20 & 10 & 0 & 10\end{array}$ 
Ethyl-4-cyclopropyl-2-oxo-2H-chromene-3-carboxylate (4I)

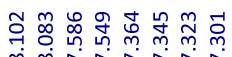

$\int_{\infty}^{\infty} \sqrt{n}$

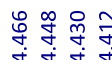

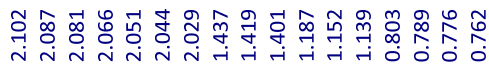

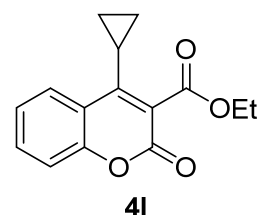

$\left({ }^{1} \mathrm{H} \mathrm{NMR}, 400 \mathrm{MHz}, \mathrm{CDCl}_{3}\right)$

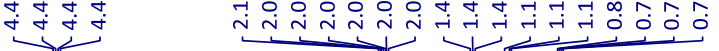

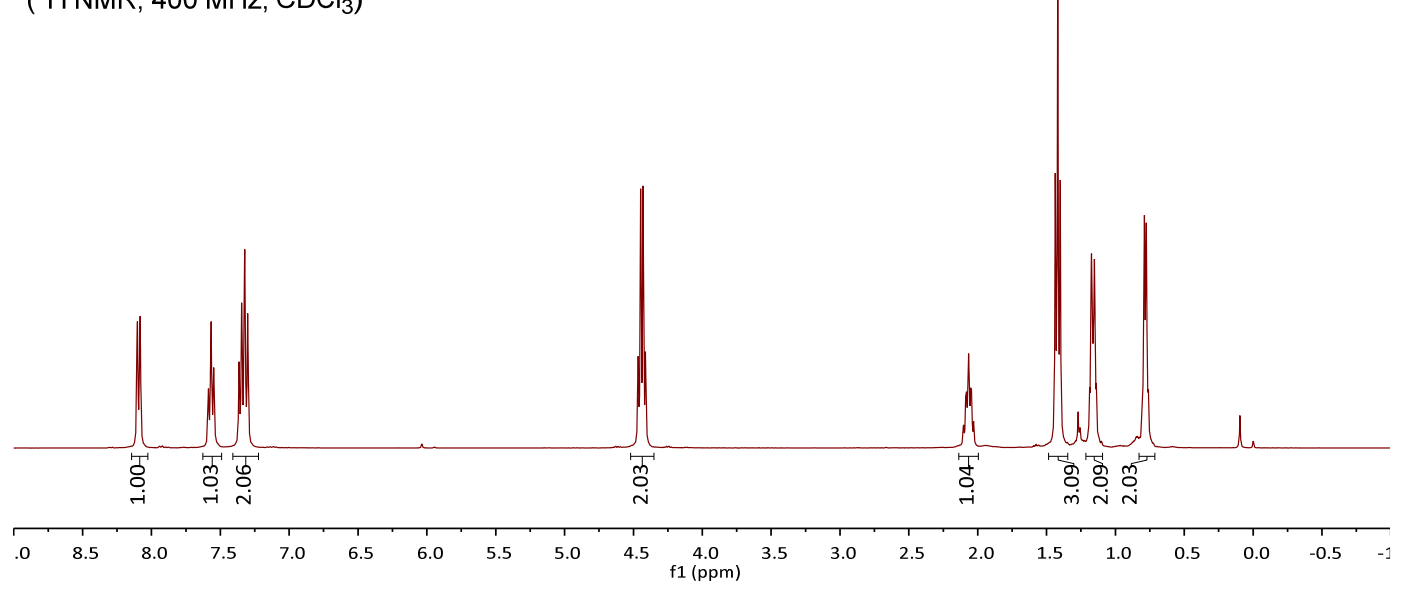

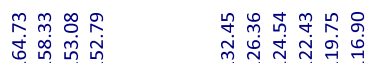

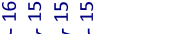

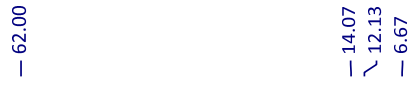<smiles>CCOC(=O)c1c(C2CC2)c2ccccc2oc1=O</smiles>

41

$\left({ }^{13} \mathrm{C} \mathrm{NMR}, 101 \mathrm{MHz}, \mathrm{CDCl}_{3}\right)$

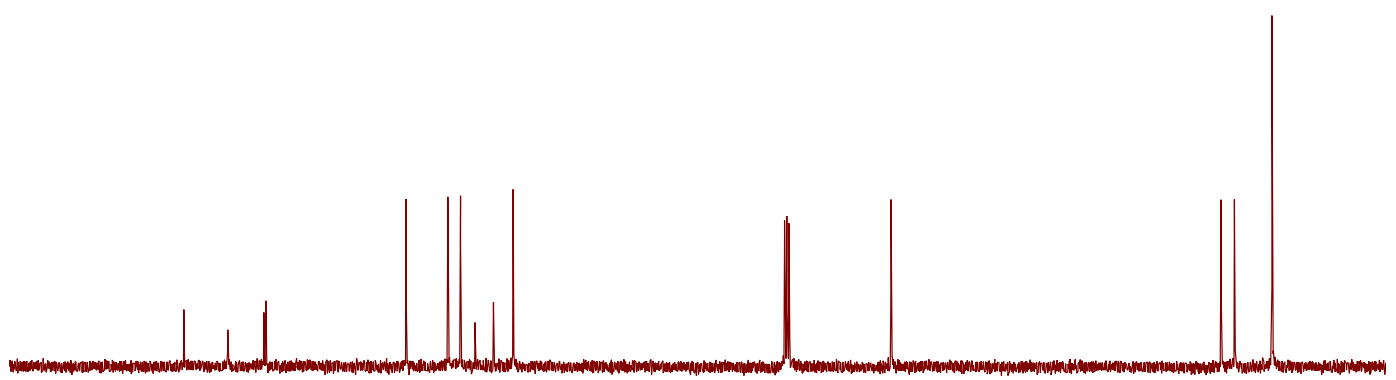

$\begin{array}{lllllllllllllllllllll}90 & 180 & 170 & 160 & 150 & 140 & 130 & 120 & 110 & 100 & 90 & 80 & 70 & 60 & 50 & 40 & 30 & 20 & 10 & 0 & -\end{array}$ 
Ethyl-4-cyclopropyl-7-methoxy-2-oxo-2H-chromene-3-carboxylate (4m)

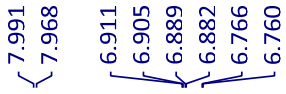

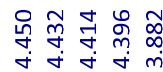

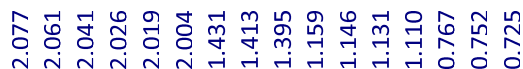<smiles>CCOC(=O)c1c(C2CC2)c2ccc(OC)cc2oc1=O</smiles>

( $\left.{ }^{1} \mathrm{H} \mathrm{NMR}, 400 \mathrm{MHz}, \mathrm{CDCl}_{3}\right)$

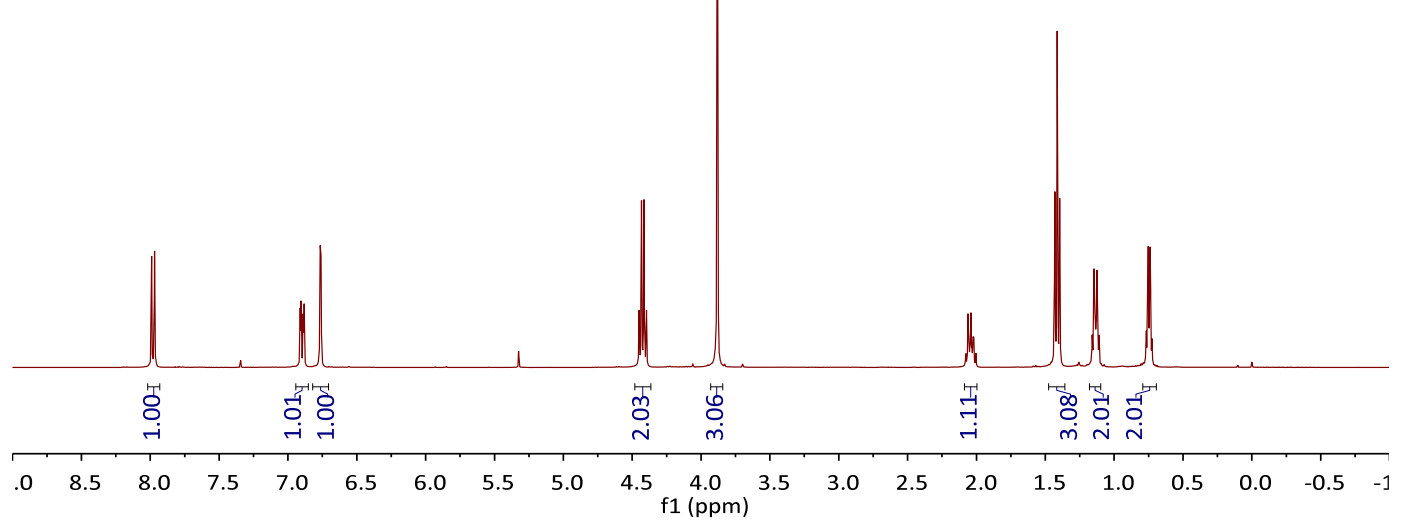

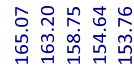

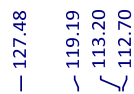

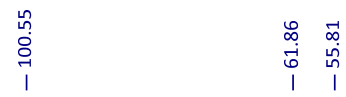

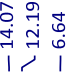

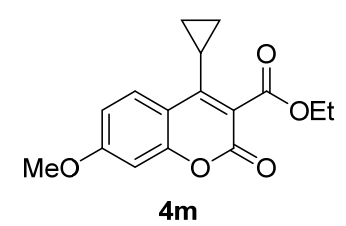

$\left({ }^{13} \mathrm{C}\right.$ NMR, $\left.101 \mathrm{MHz}, \mathrm{CDCl}_{3}\right)$

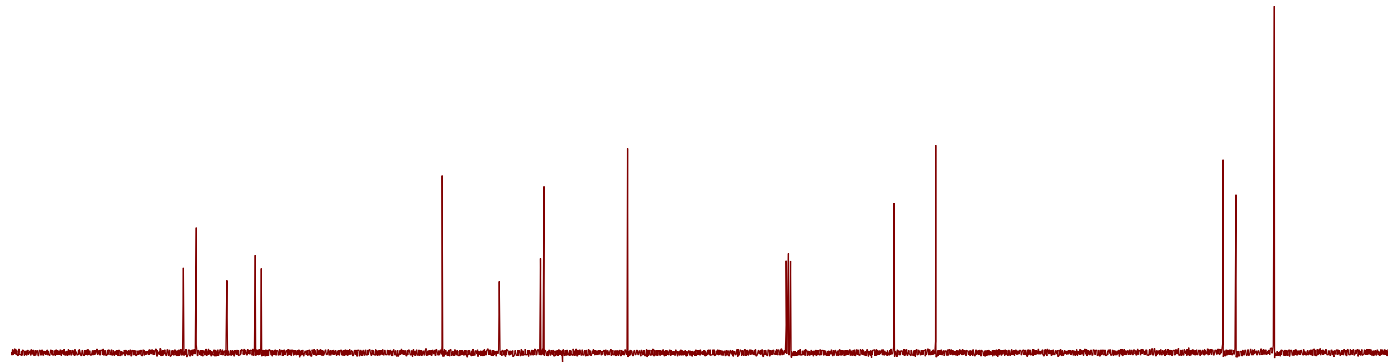

$\begin{array}{llllllllllllllllllll}90 & 180 & 170 & 160 & 150 & 140 & 130 & 120 & 110 & 100 & 90 & 80 & 70 & 60 & 50 & 40 & 30 & 20 & 10 & 0\end{array}$ 
Ethyl-4-cyclopropyl-6-methoxy-2-oxo-2H-chromene-3-carboxylate (4n)

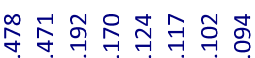

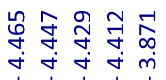

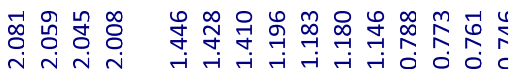

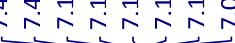

irir

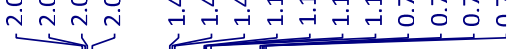<smiles>CCOC(=O)c1c(C2CC2)c2cc(OC)ccc2oc1=O</smiles>

( ${ }^{1} \mathrm{H} \mathrm{NMR}, 400 \mathrm{MHz}, \mathrm{CDCl}_{3}$ )

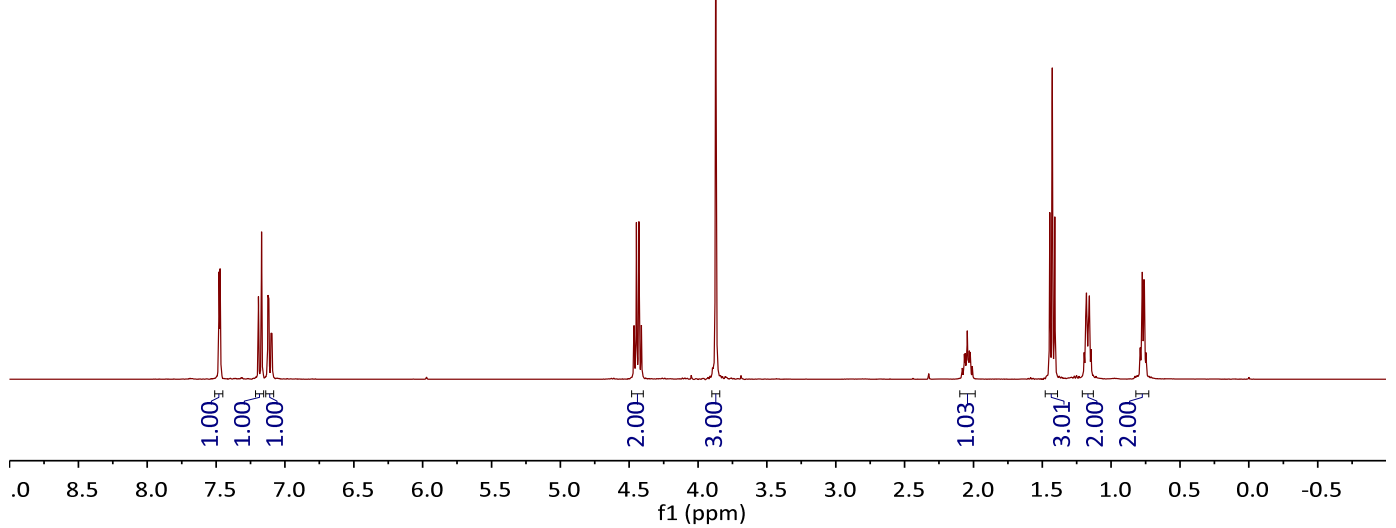

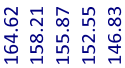

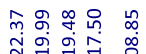
光寻

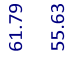

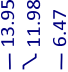

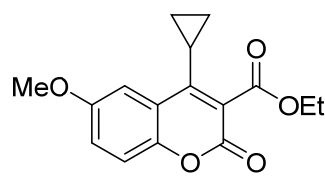

4n

$\left({ }^{13} \mathrm{C} \mathrm{NMR}, 101 \mathrm{MHz}, \mathrm{CDCl}_{3}\right)$

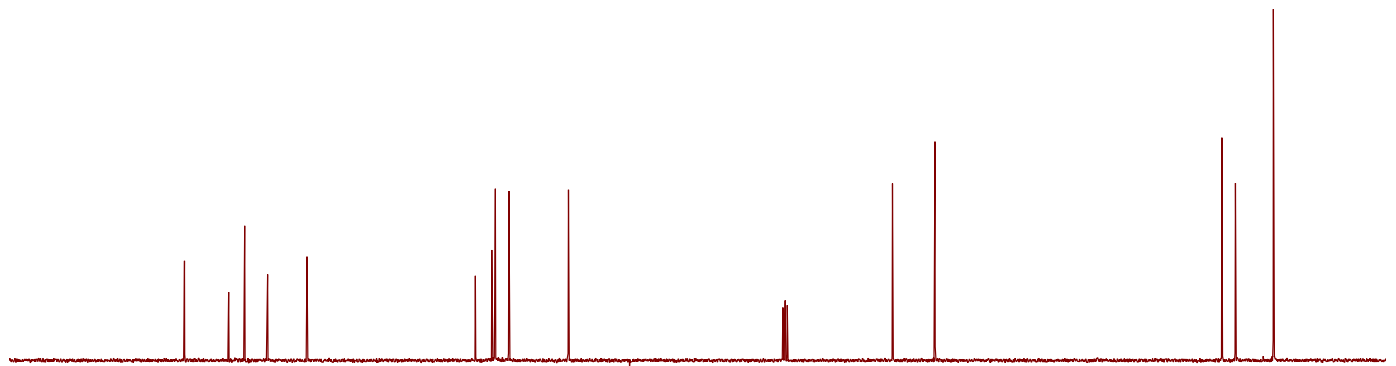

$\begin{array}{llllllllllllllllllll}90 & 180 & 170 & 160 & 150 & 140 & 130 & 120 & 110 & 100 & 90 & 80 & 70 & 60 & 50 & 40 & 30 & 20 & 10 & 0\end{array}$ 
Ethyl 2-oxo-4-phenyl-2H-chromene-3-carboxylate (40)

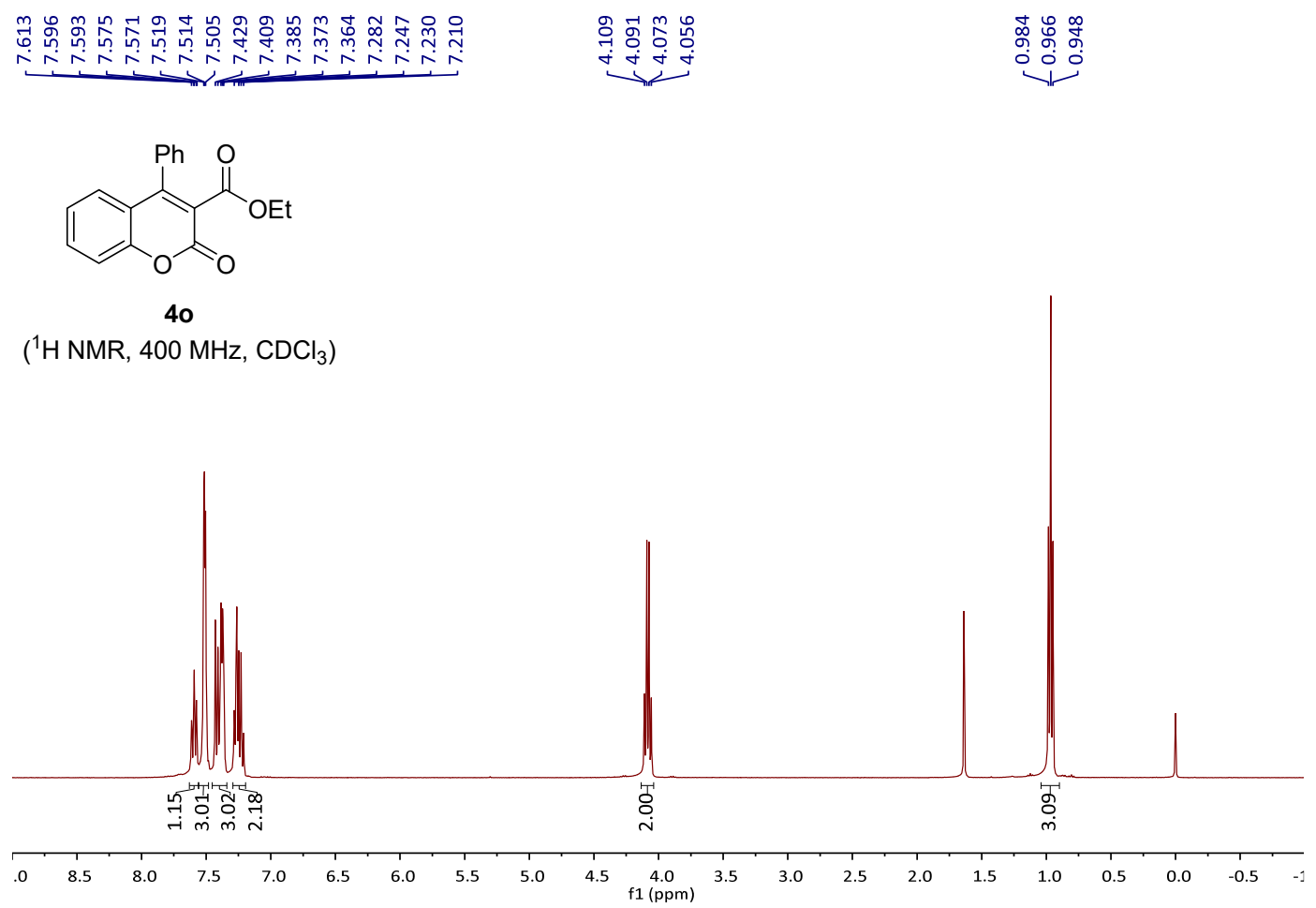

(+)-Ethyl 1,4-dimethyl-2-oxo-1,2,3,4-tetrahydroquinoline-3-carboxylate (3a)

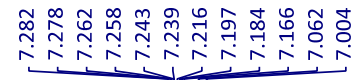

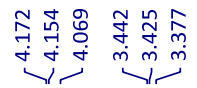

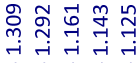

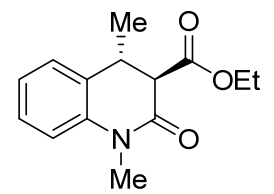

(+)-3a

$\left({ }^{1} \mathrm{H} \mathrm{NMR}, 400 \mathrm{MHz}, \mathrm{CDCl}_{3}\right)$

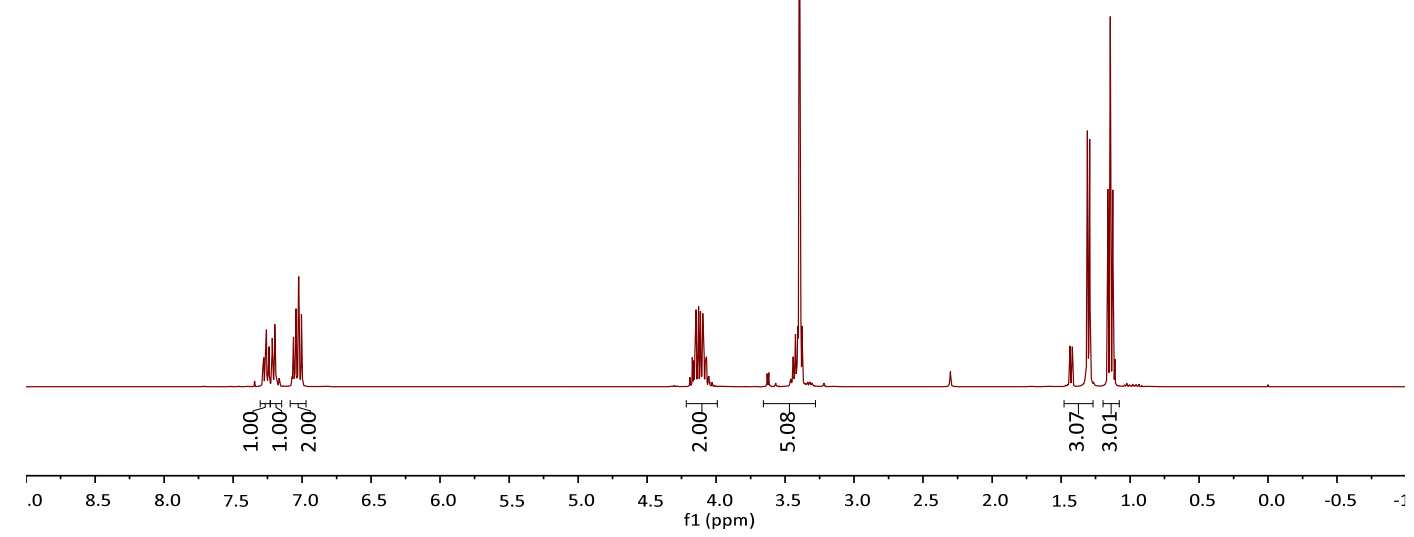




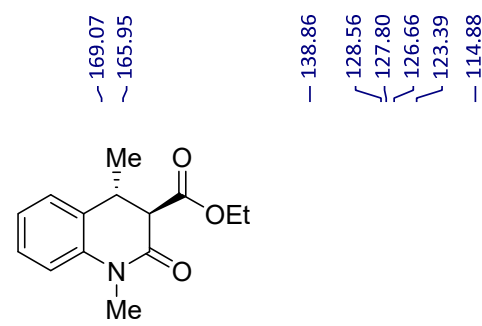

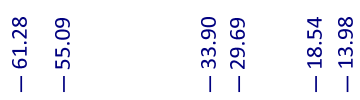

(+)-3a

$\left({ }^{13} \mathrm{C} \mathrm{NMR}, 101 \mathrm{MHz}, \mathrm{CDCl}_{3}\right)$

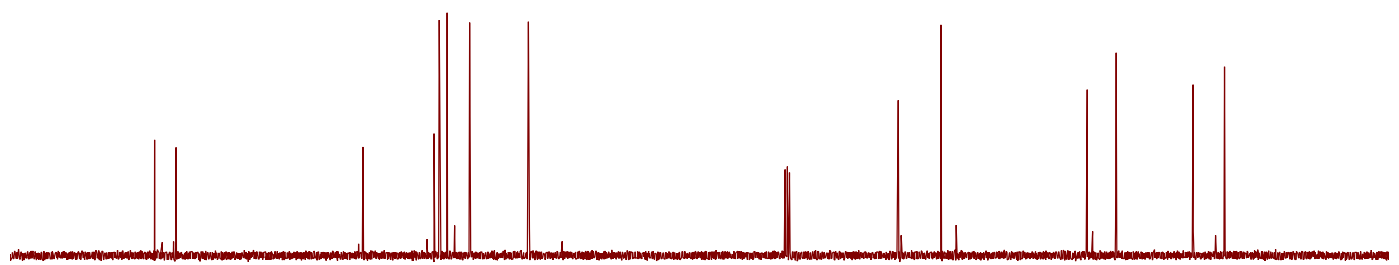

$\begin{array}{lllllllllllllllllllll}90 & 180 & 170 & 160 & 150 & 140 & 130 & 120 & 110 & 100 & 90 & 80 & 70 & 60 & 50 & 40 & 30 & 20 & 10 & 0\end{array}$ -

(-)-Ethyl 4-ethyl-1-methyl-2-oxo-1,2,3,4-tetrahydroquinoline-3-carboxylate (3b)
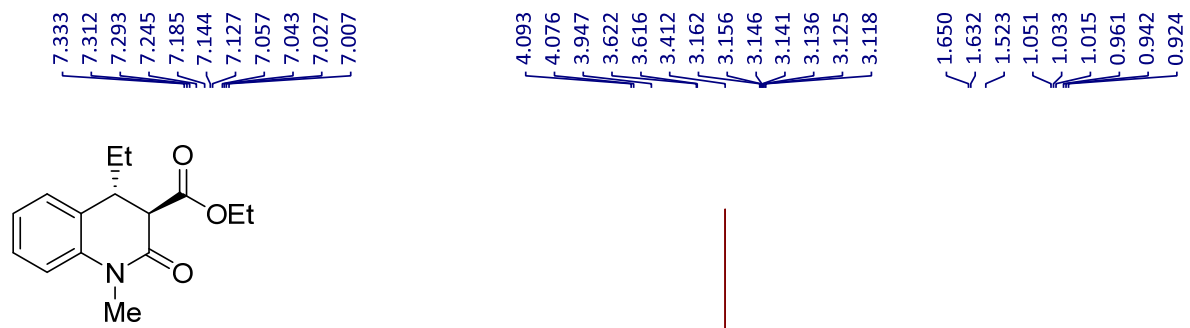

$(-)-3 b$

$\left({ }^{1} \mathrm{H}\right.$ NMR, $\left.400 \mathrm{MHz}, \mathrm{CDCl}_{3}\right)$

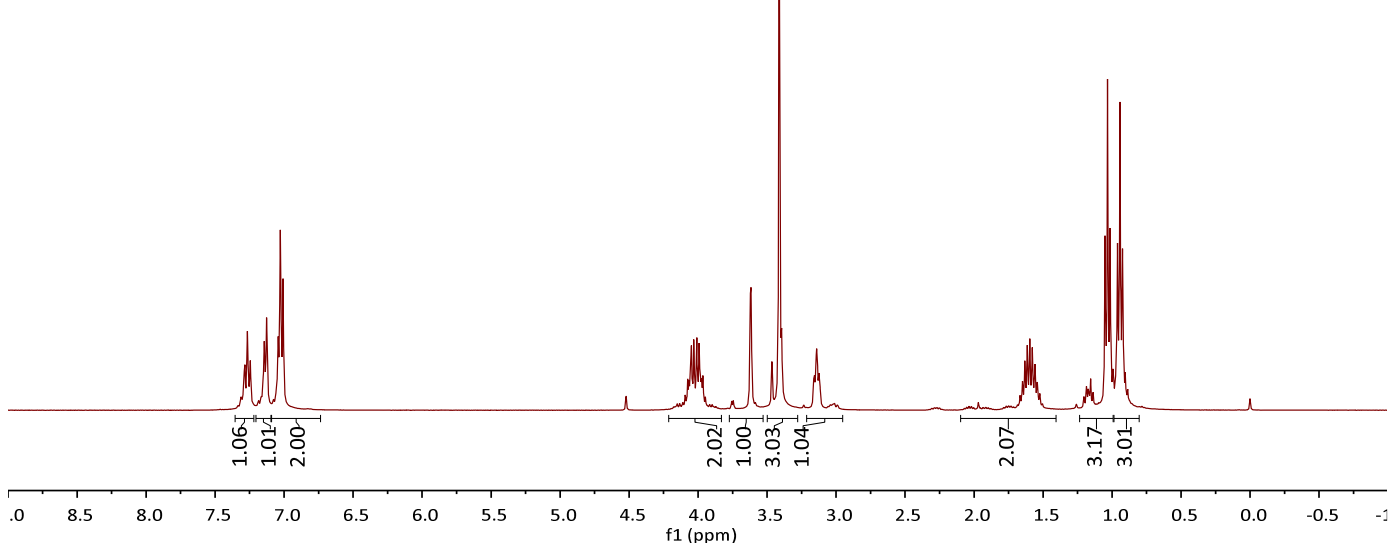



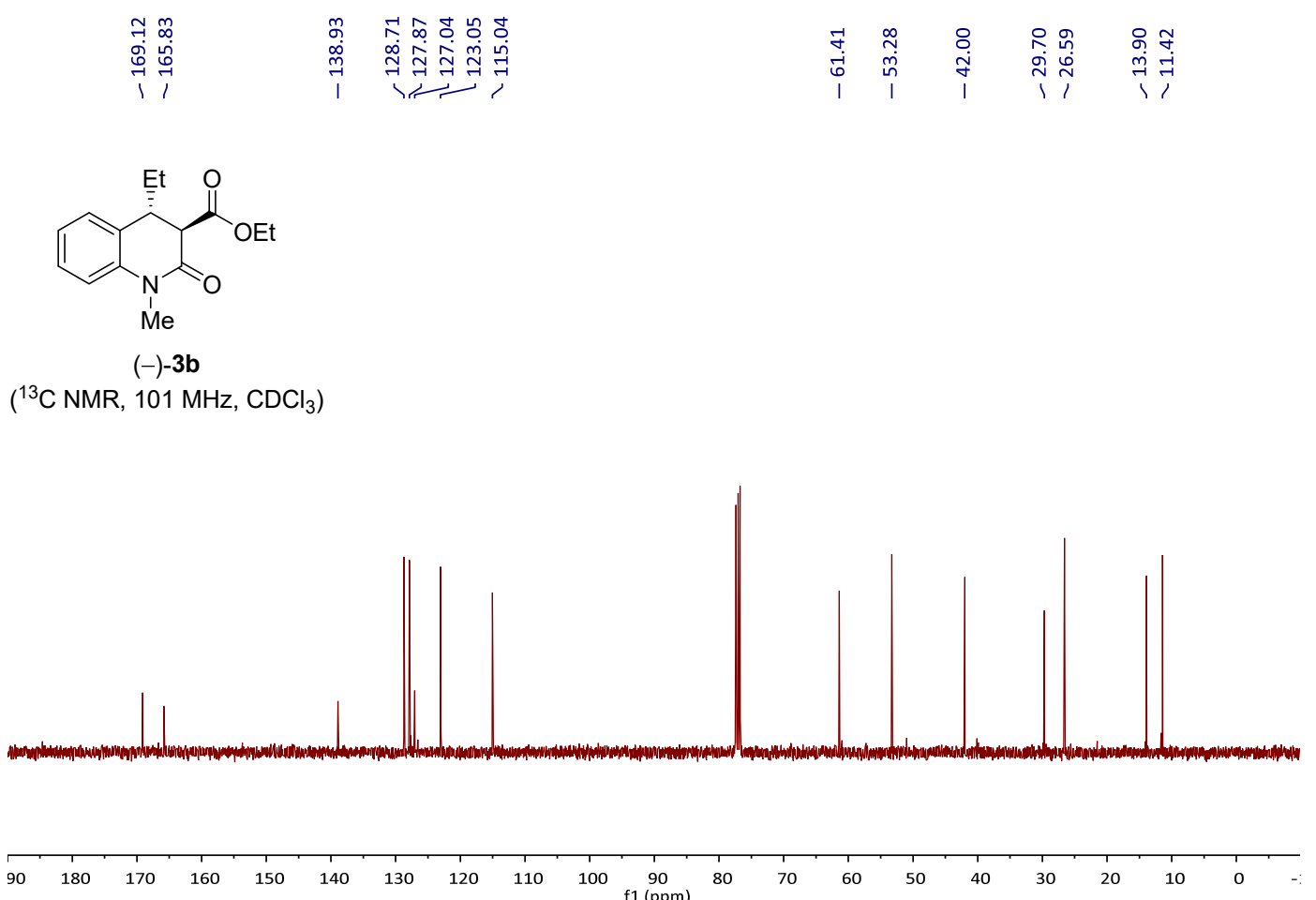

(+)-Ethyl 1-methyl-2-oxo-4-propyl-1,2,3,4-tetrahydroquinoline-3-carboxylate (3c)

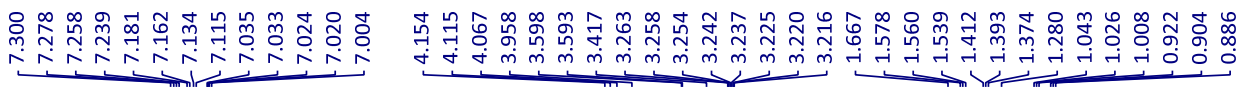

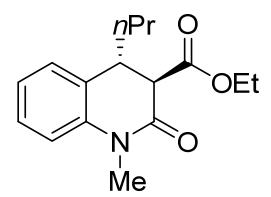

(+)-3c

$\left({ }^{1} \mathrm{H} \mathrm{NMR}, 400 \mathrm{MHz}, \mathrm{CDCl}_{3}\right)$

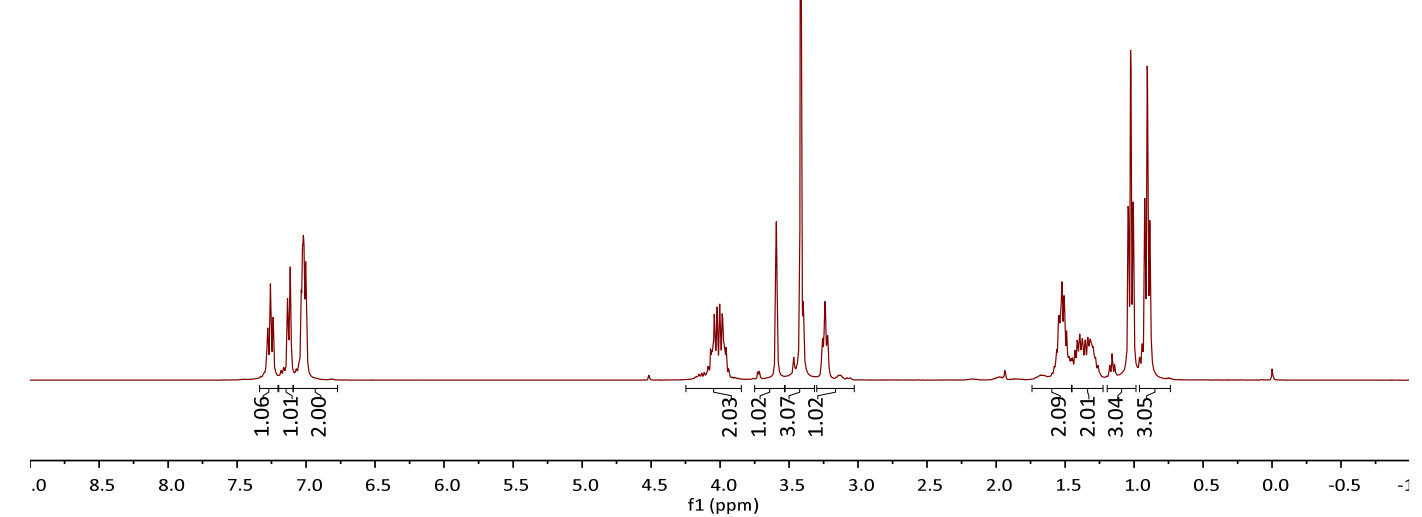




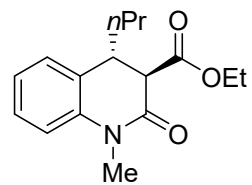

$(+)-3 c$

$\left({ }^{13} \mathrm{C} \mathrm{NMR}, 101 \mathrm{MHz}, \mathrm{CDCl}_{3}\right)$
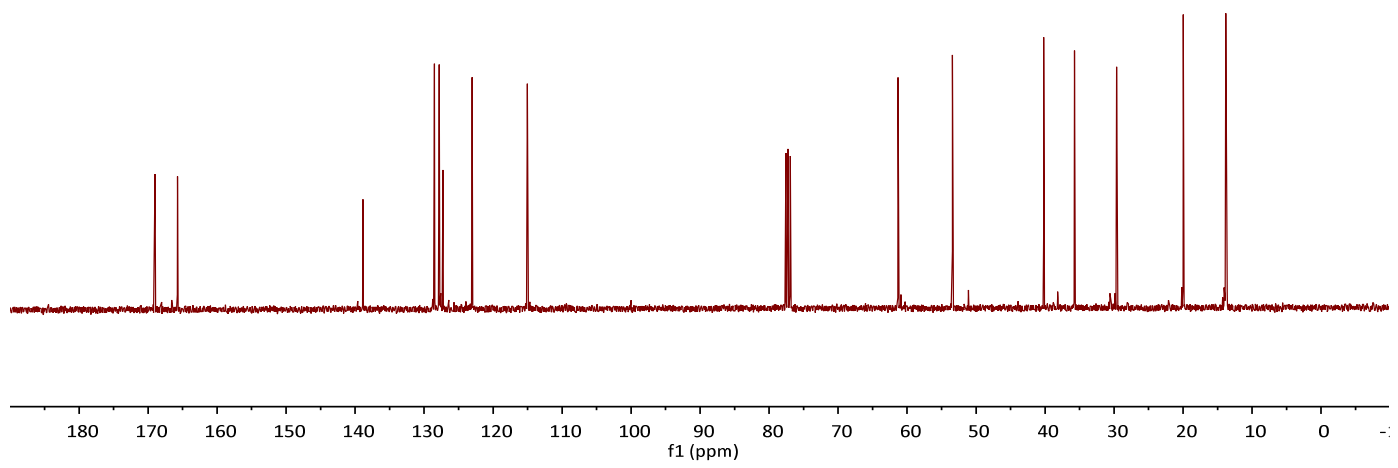

(+)-Ethyl 4-isobutyl-1-methyl-2-oxo-1,2,3,4-tetrahydroquinoline-3-carboxylate (3d)

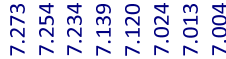

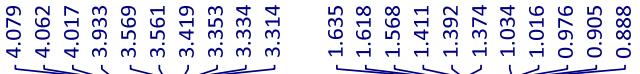<smiles>CCOC(=O)C1C(=O)N([N+](=O)[O-])c2ccccc2[C@H]1Br</smiles>

$(+)-3 \mathbf{d}$

$\left({ }^{1} \mathrm{H} \mathrm{NMR}, 400 \mathrm{MHz}, \mathrm{CDCl}_{3}\right)$

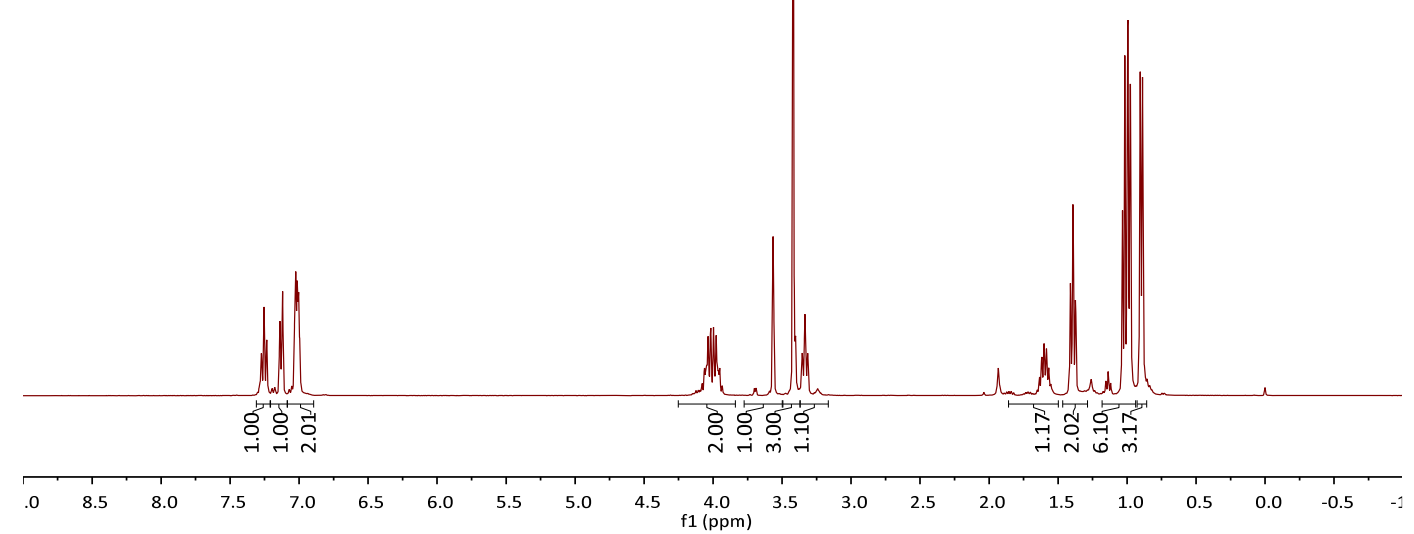




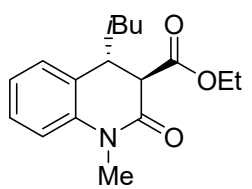

(+)-3d

$\left({ }^{13} \mathrm{C} \mathrm{NMR}, 101 \mathrm{MHz}, \mathrm{CDCl}_{3}\right)$
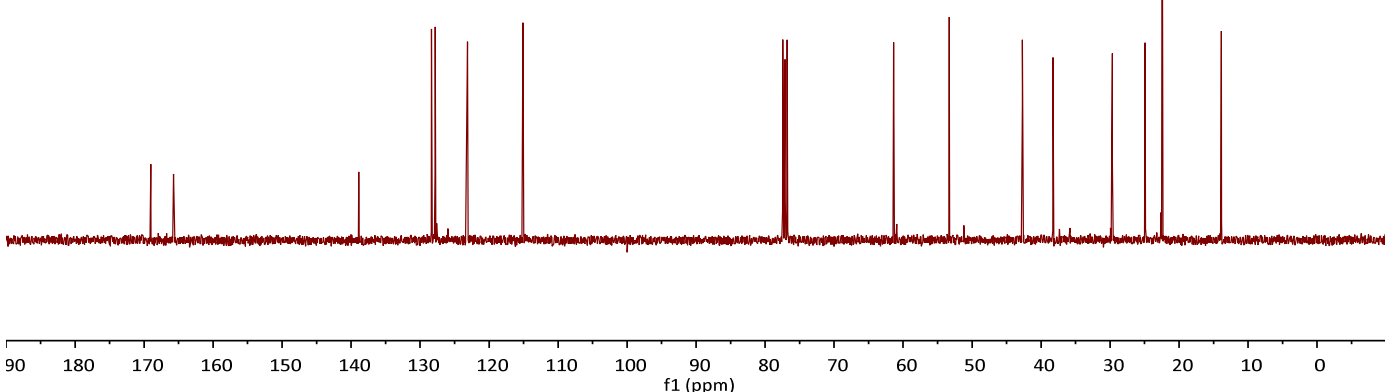

(+)-Ethyl 4-benzyl-1-methyl-2-oxo-1,2,3,4-tetrahydroquinoline-3-carboxylate (3e)

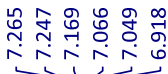

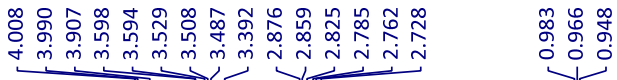<smiles>CCOC(=O)C1C(=O)N([N+](=O)[O-])c2ccccc2C1Cc1ccccc1</smiles>

$(+)-3 e$

$\left({ }^{1} \mathrm{HNMR}, 400 \mathrm{MHz}, \mathrm{CDCl}_{3}\right)$

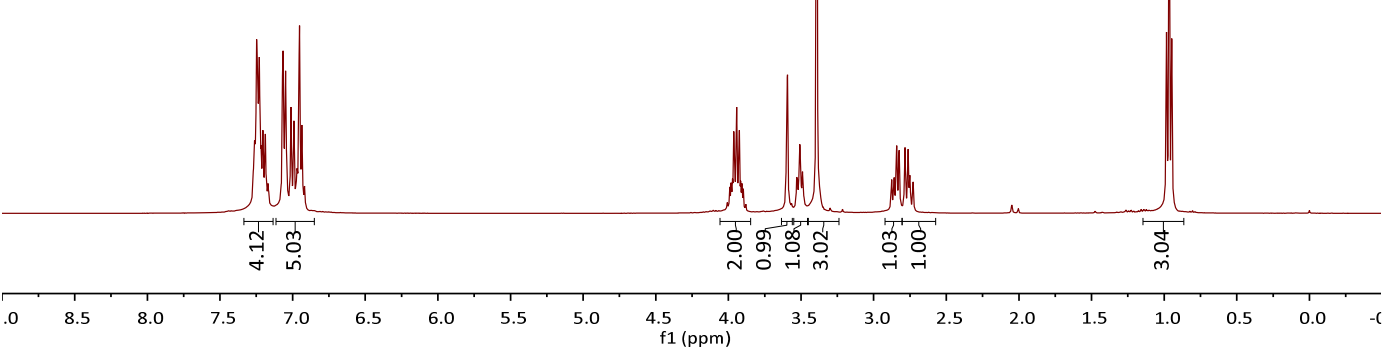




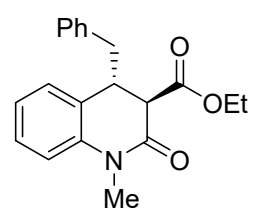

(+)-3e

$\left({ }^{13} \mathrm{C}\right.$ NMR, $\left.101 \mathrm{MHz}, \mathrm{CDCl}_{3}\right)$
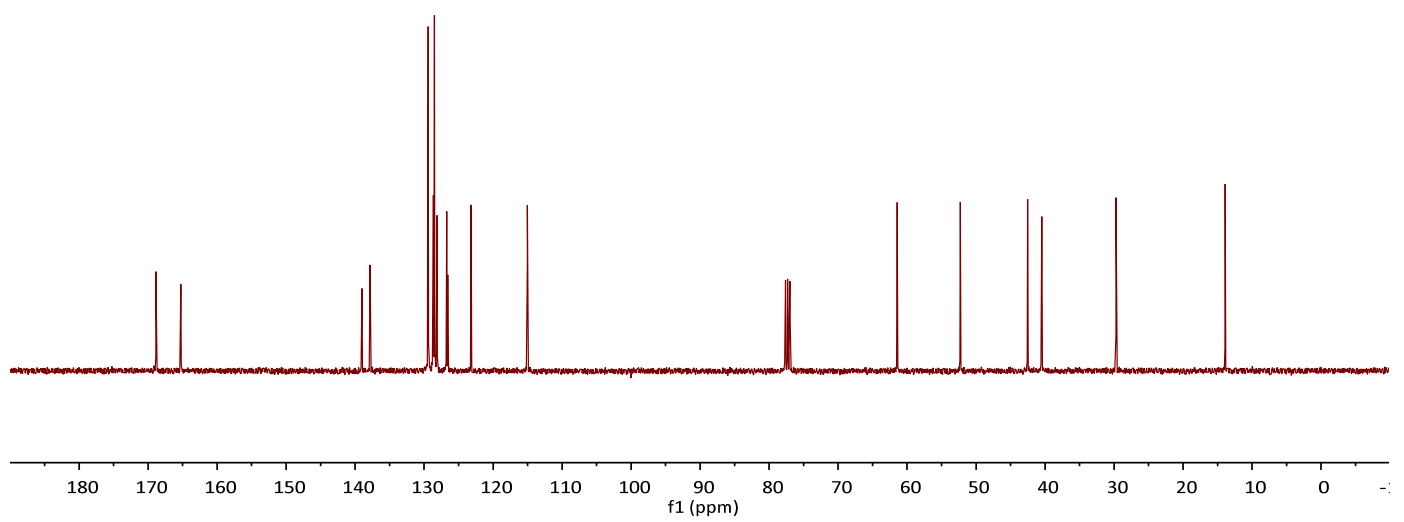

(+)-Ethyl 1-methyl-2-oxo-4-phenethyl-1,2,3,4-tetrahydroquinoline-3-carboxylate (3f)

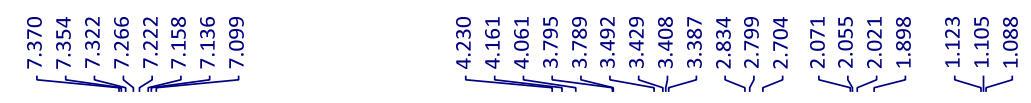<smiles>CCOC(=O)C1C[C@@H](CCc2ccccc2)N([N+](=O)c2ccccc2)C1=O</smiles>

$(+)-3 \mathbf{f}$

( $\left.{ }^{1} \mathrm{H} \mathrm{NMR}, 400 \mathrm{MHz}, \mathrm{CDCl}_{3}\right)$

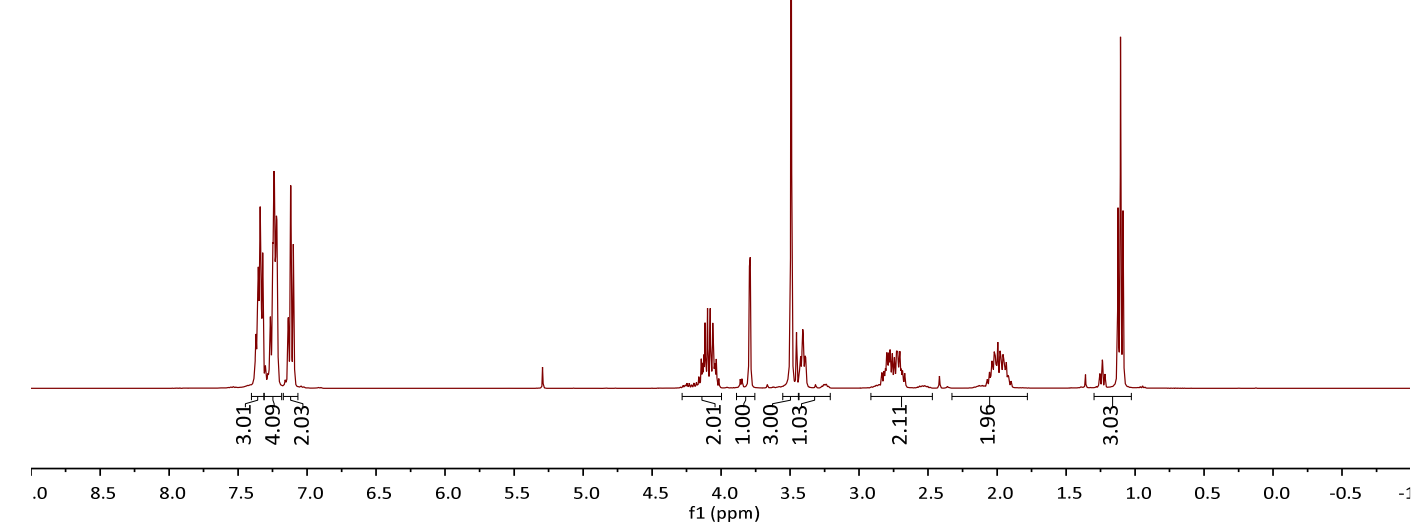


<smiles>CCOC(=O)C1C(=O)N(C)c2ccccc2[C@H]1CCc1ccccc1</smiles>

$(+)-3 \mathbf{f}$

$\left({ }^{13} \mathrm{C} \mathrm{NMR}, 101 \mathrm{MHz}, \mathrm{CDCl}_{3}\right)$

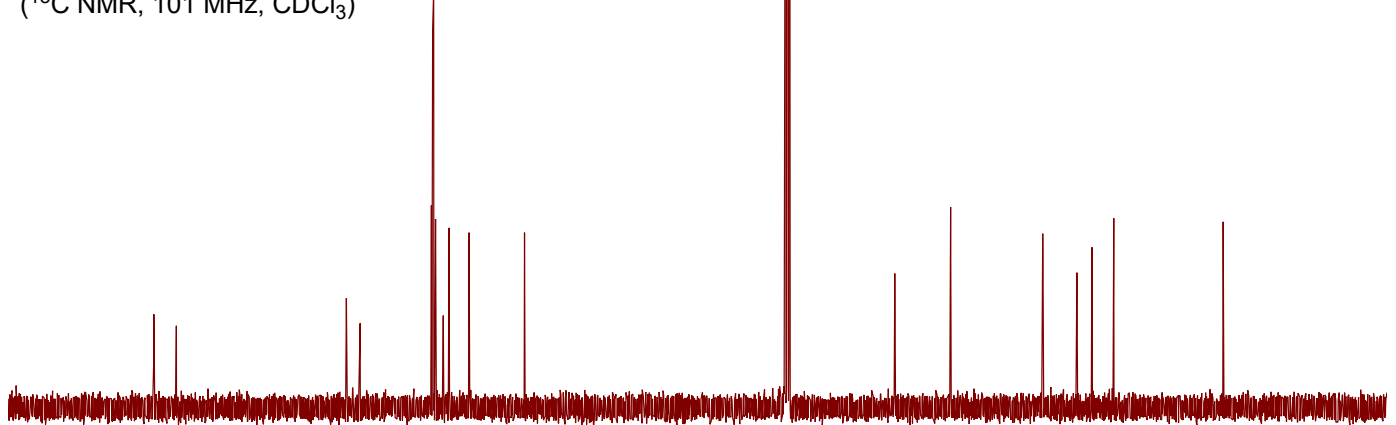

$\begin{array}{lllllllllllllllllllll}90 & 180 & 170 & 160 & 150 & 140 & 130 & 120 & 110 & 100 & 90 & 80 & 70 & 60 & 50 & 40 & 30 & 20 & 10 & 0 & -\end{array}$

(+)-Ethyl 1-methyl-2-oxo-4-pentyl-1,2,3,4-tetrahydroquinoline-3-carboxylate (3g)

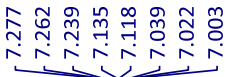
$\underbrace{r}$<smiles>CCOC(=O)C1C(=O)N([N+](=O)[O-])c2ccccc2C1CC(C)C</smiles>

$(+)-3 g$

$\left({ }^{1} \mathrm{H} \mathrm{NMR}, 400 \mathrm{MHz}, \mathrm{CDCl}_{3}\right)$

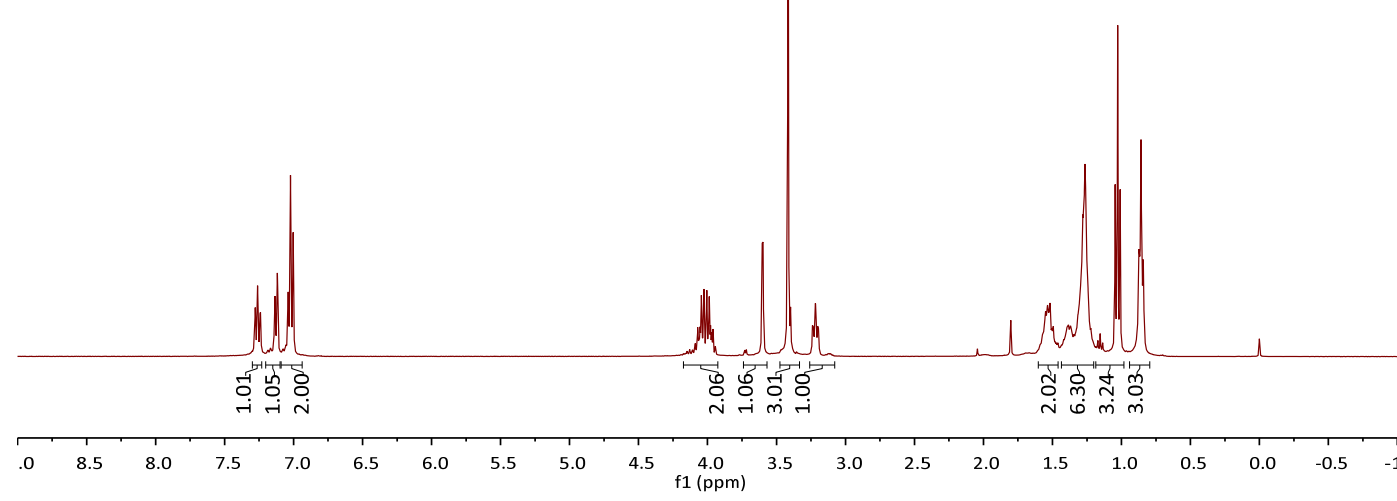




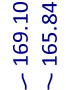

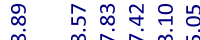

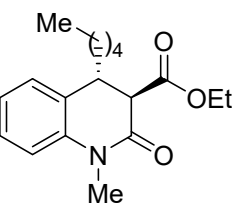

$(+)-3 g$

$\left({ }^{13} \mathrm{C} \mathrm{NMR}, 101 \mathrm{MHz}, \mathrm{CDCl}_{3}\right)$

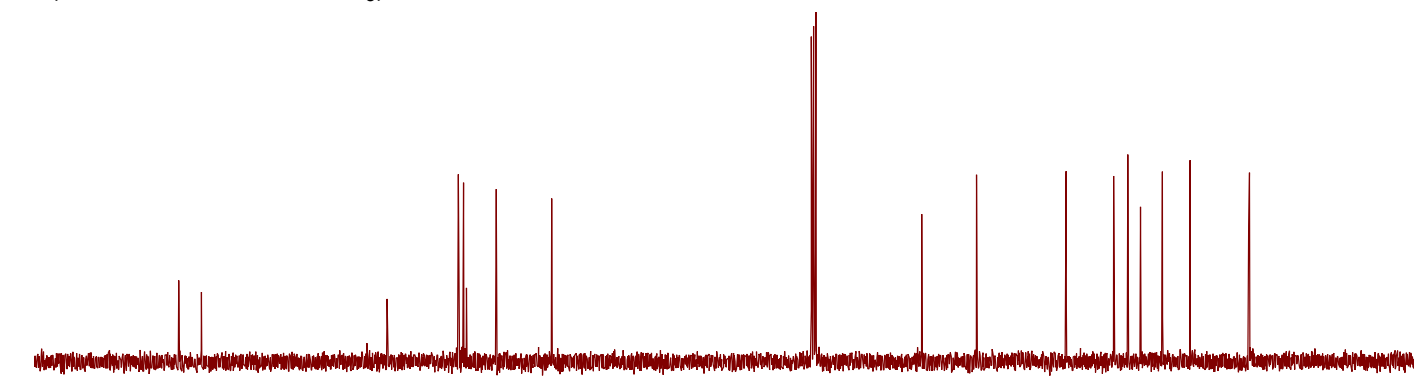

$180 \quad 170$
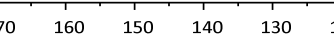

(+)-Ethyl 4-cyclopropyl-1-methyl-2-oxo-1,2,3,4-tetrahydroquinoline-3-carboxylate (3h)

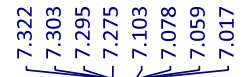

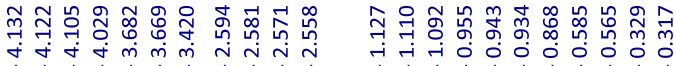<smiles>CCOC(=O)C1C(=O)N([N+](=O)[O-])c2ccccc2[C@H]1C1CC1</smiles>

$(+)-3 \mathrm{~h}$

$\left({ }^{1} \mathrm{H} \mathrm{NMR}, 400 \mathrm{MHz}, \mathrm{CDCl}_{3}\right)$

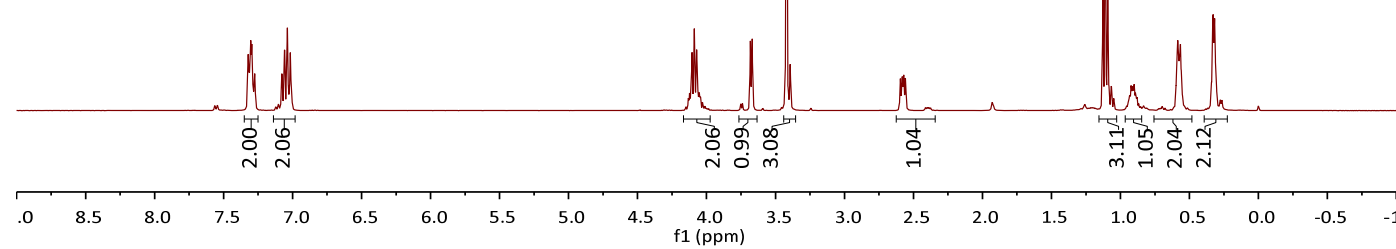




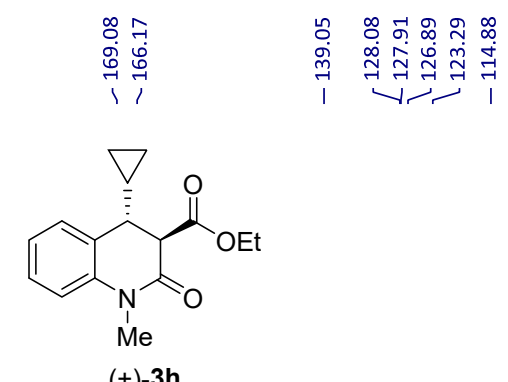

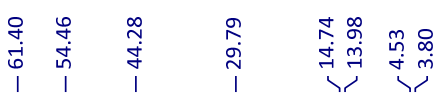

$\left({ }^{13} \mathrm{C}\right.$ NMR, $\left.101 \mathrm{MHz}, \mathrm{CDCl}_{3}\right)$

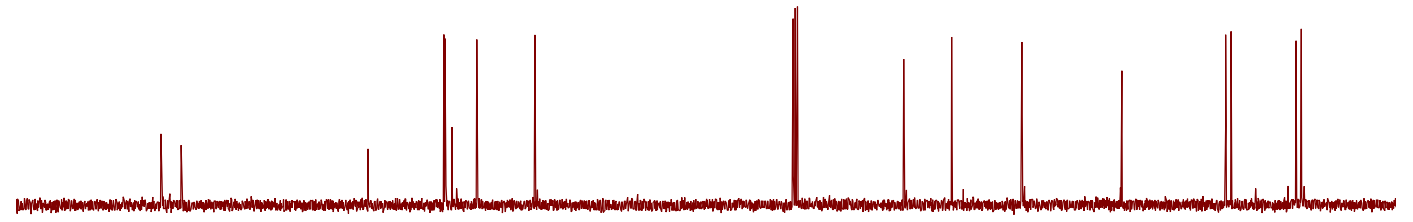

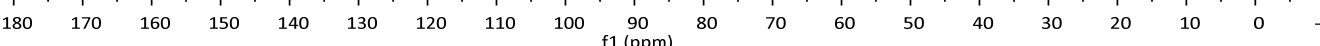

(-)-(3S,4R)-Ethyl 1-methyl-2-oxo-4-phenyl-1,2,3,4-tetrahydroquinoline-3-carboxylate ((3S,4R)-3i)

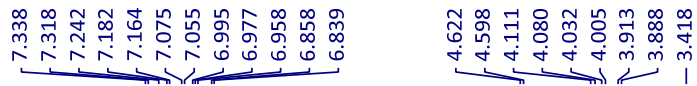

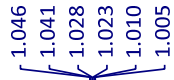<smiles>CCOC(=O)[C@H]1C(=O)N(C)c2ccccc2[C@H]1c1ccccc1</smiles>

(3S,4R)-3i

$\left({ }^{1} \mathrm{H} \mathrm{NMR}, 400 \mathrm{MHz}, \mathrm{CDCl}_{3}\right)$

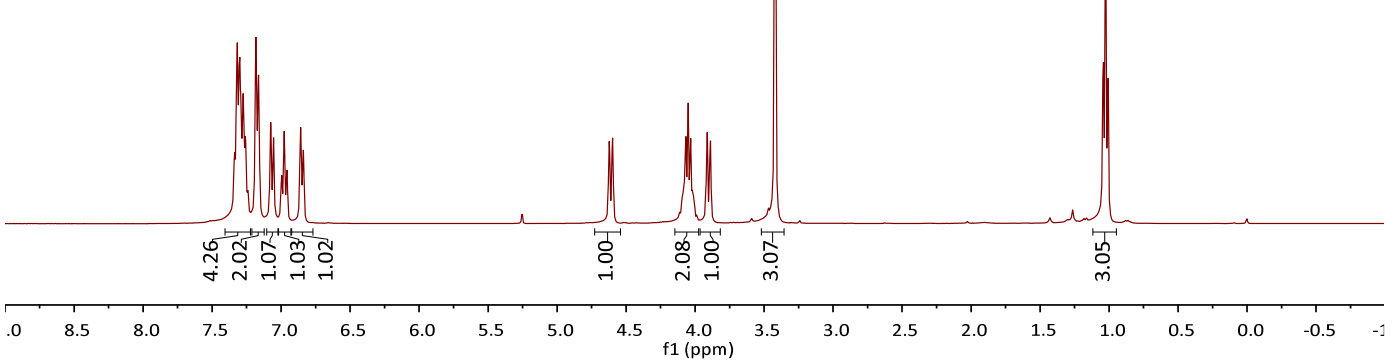




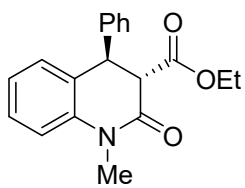

$(3 S, 4 R)-\mathbf{3 i}$

$\left({ }^{13} \mathrm{C}\right.$ NMR, $\left.101 \mathrm{MHz}, \mathrm{CDCl}_{3}\right)$
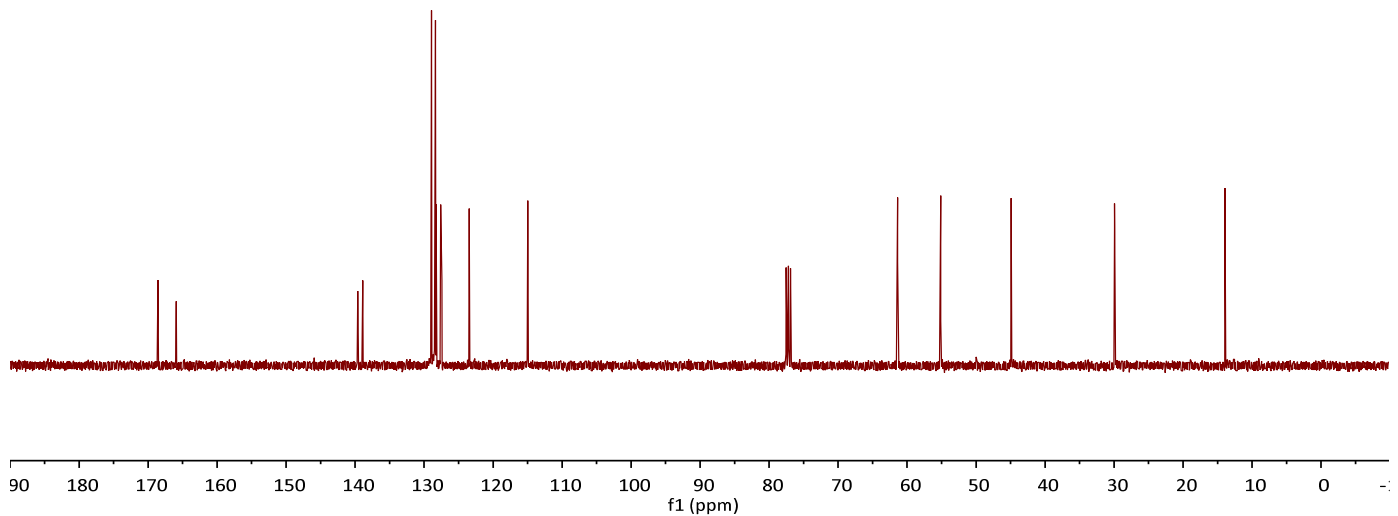

(+)-Ethyl 6-bromo-1,4-dimethyl-2-oxo-1,2,3,4-tetrahydroquinoline-3-carboxylate (3j)

品

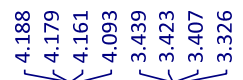

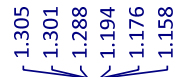<smiles>CCOC(=O)[C@H]1C(=O)N(C)c2ccc(Br)cc2[C@@H]1C</smiles>

$(+)-3 \mathbf{j}$

$\left({ }^{1} \mathrm{H} \mathrm{NMR}, 400 \mathrm{MHz}, \mathrm{CDCl}_{3}\right.$ )

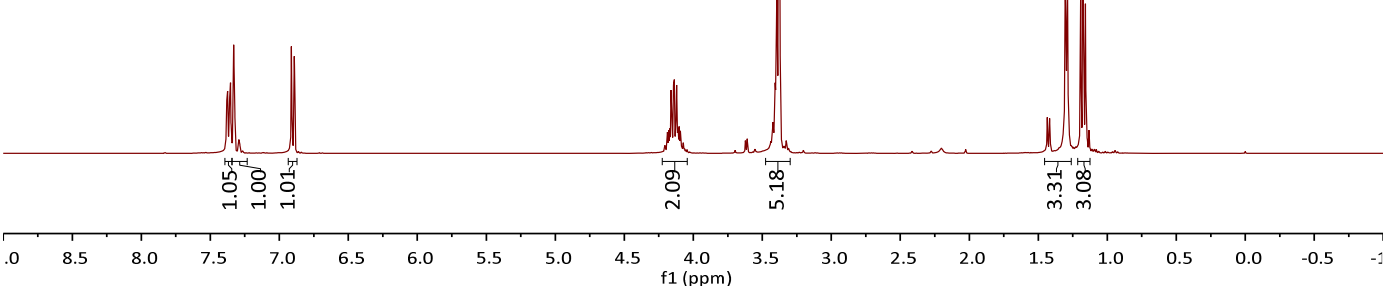




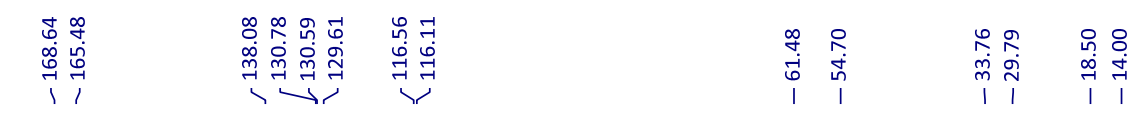

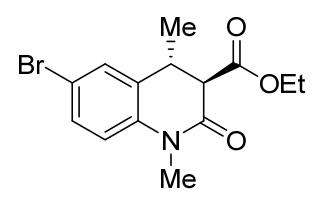

$(+)-3 \mathbf{j}$

$\left({ }^{13} \mathrm{C} \mathrm{NMR,}, 101 \mathrm{MHz}, \mathrm{CDCl}_{3}\right)$

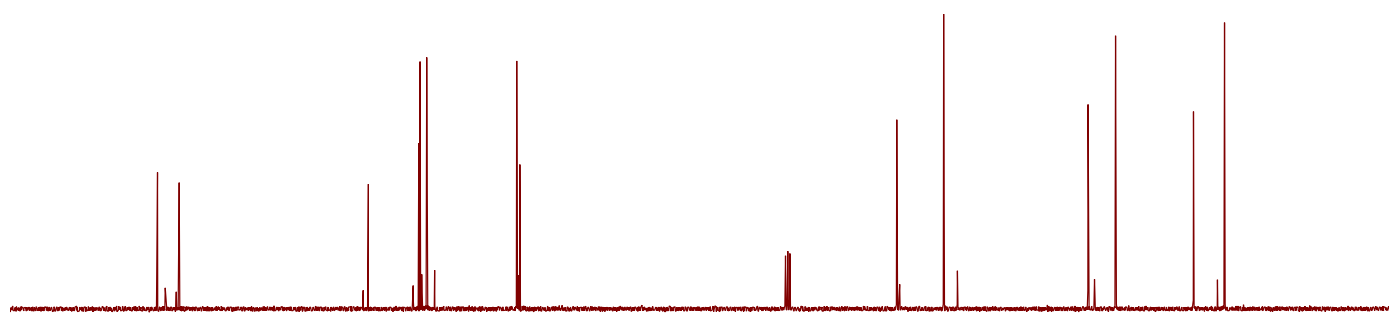

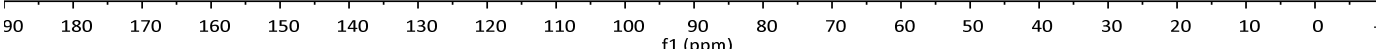

(-)-Ethyl 6-methoxy-1,4-dimethyl-2-oxo-1,2,3,4-tetrahydroquinoline-3-carboxylate (3k)

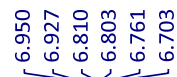

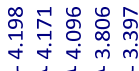

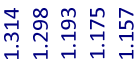

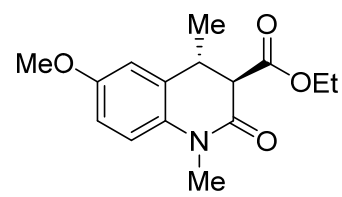

$(-)-3 \mathbf{k}$

$\left({ }^{1} \mathrm{H} N M R, 400 \mathrm{MHz}, \mathrm{CDCl}_{3}\right)$

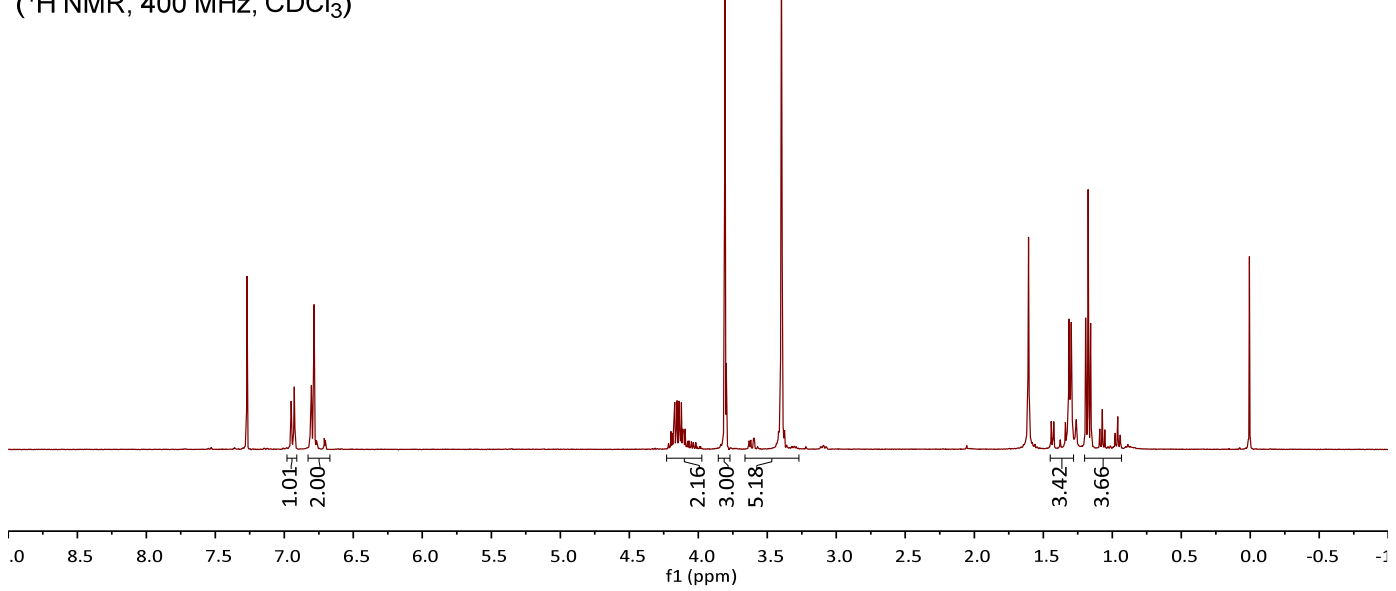




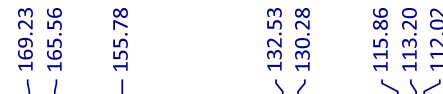

รั กู่

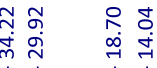

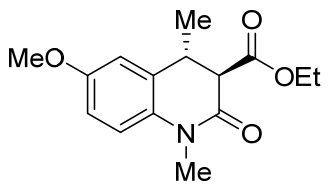

$(-)-3 k$

$\left({ }^{13} \mathrm{C} \mathrm{NMR}, 101 \mathrm{MHz}, \mathrm{CDCl}_{3}\right)$

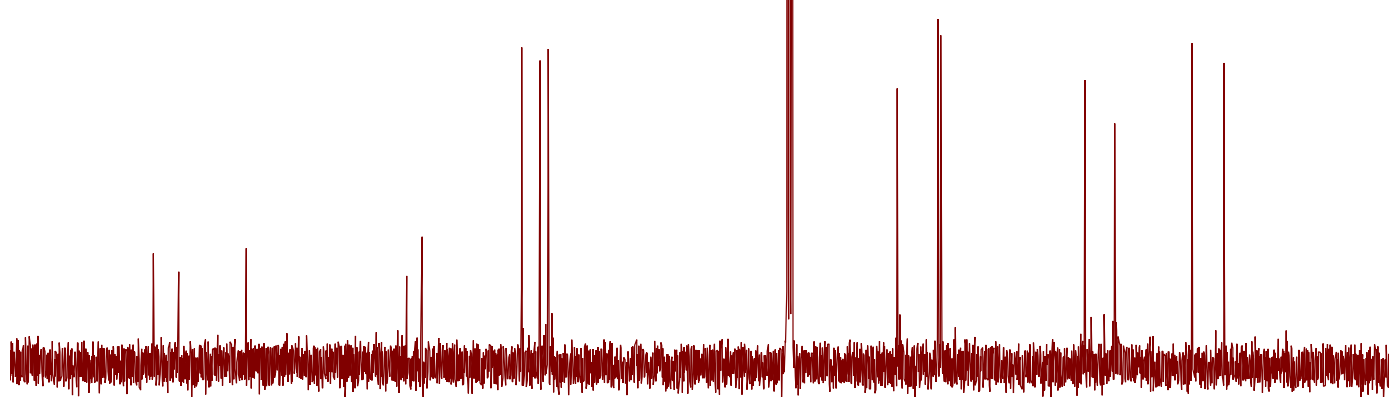

\begin{tabular}{llllllllllllllllllll}
\hline 0 & 180 & 170 & 160 & 150 & 140 & 130 & 120 & 110 & 100 & $\underset{\mathrm{f} 1}{90}(\mathrm{ppm})$ & 80 & 70 & 60 & 50 & 40 & 30 & 20 & 10 & 0
\end{tabular}

(+)-Ethyl 1-ethyl-4-methyl-2-oxo-1,2,3,4-tetrahydroquinoline-3-carboxylate (3I)
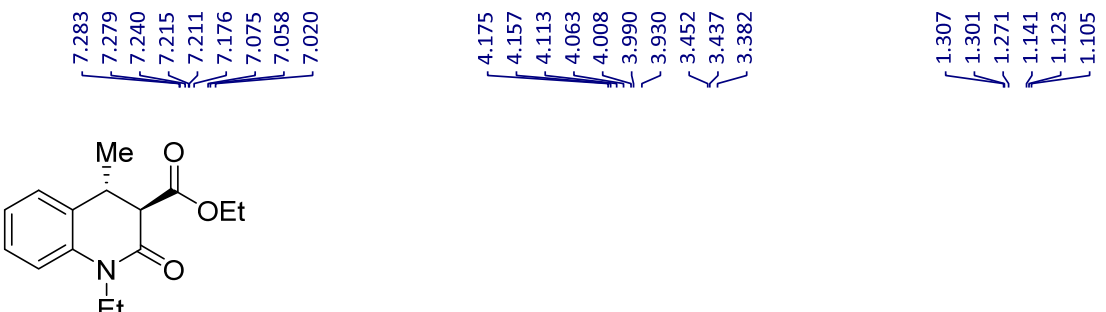

$(+)-31$

$\left({ }^{1} \mathrm{H} \mathrm{NMR}, 400 \mathrm{MHz}, \mathrm{CDCl}_{3}\right)$

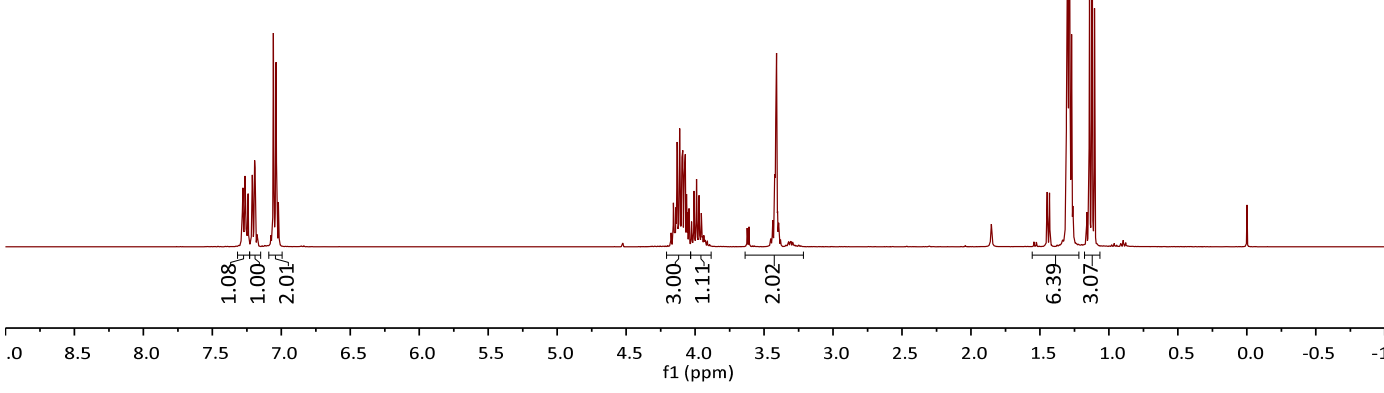




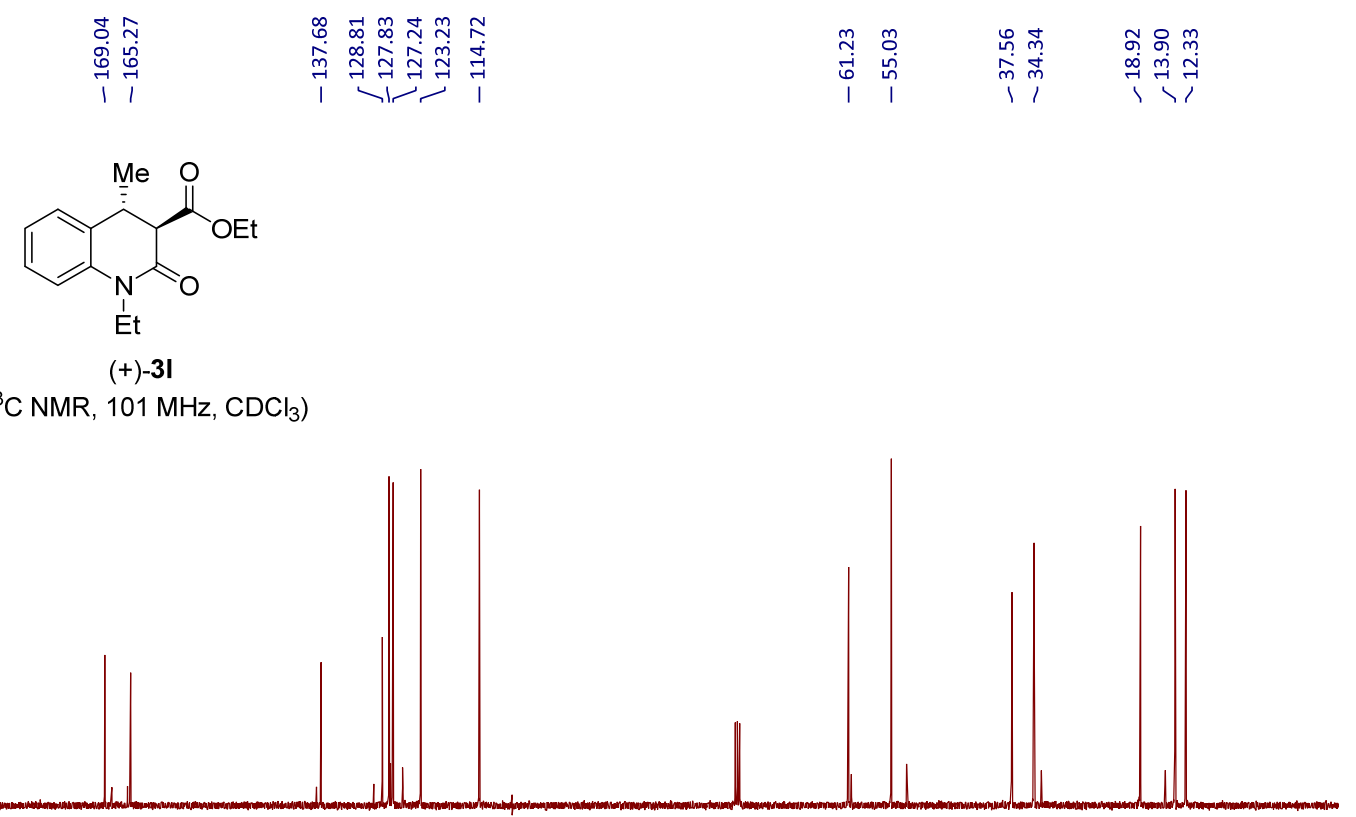

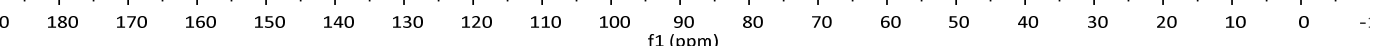

(+)-Ethyl 1-benzyl-4-methyl-2-oxo-1,2,3,4-tetrahydroquinoline-3-carboxylate (3m)

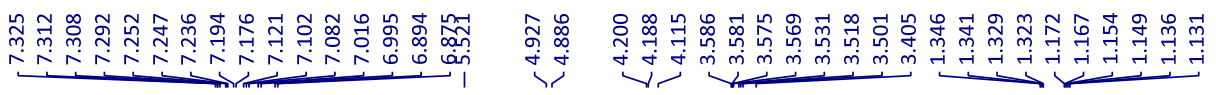<smiles>CCOC(=O)C1C(=O)N(Cc2ccccc2)c2ccccc2C1(C)C</smiles>

$(+)-3 m$

$\left({ }^{1} \mathrm{HNMR}, 400 \mathrm{MHz}, \mathrm{CDCl}_{3}\right)$

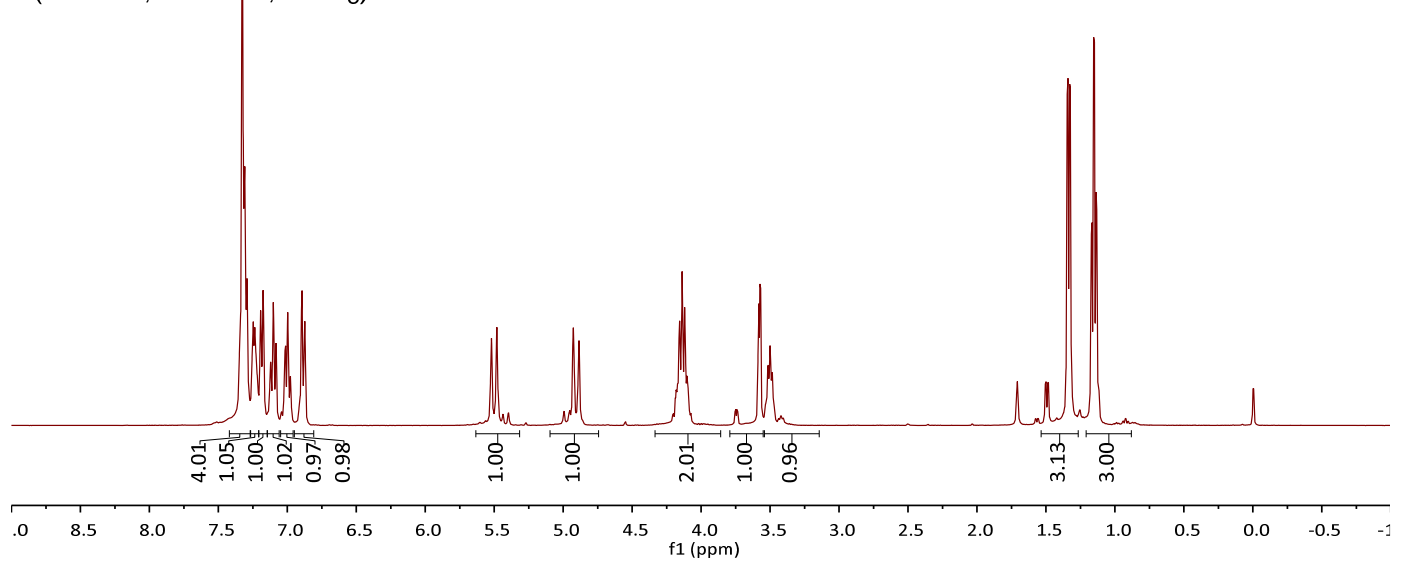




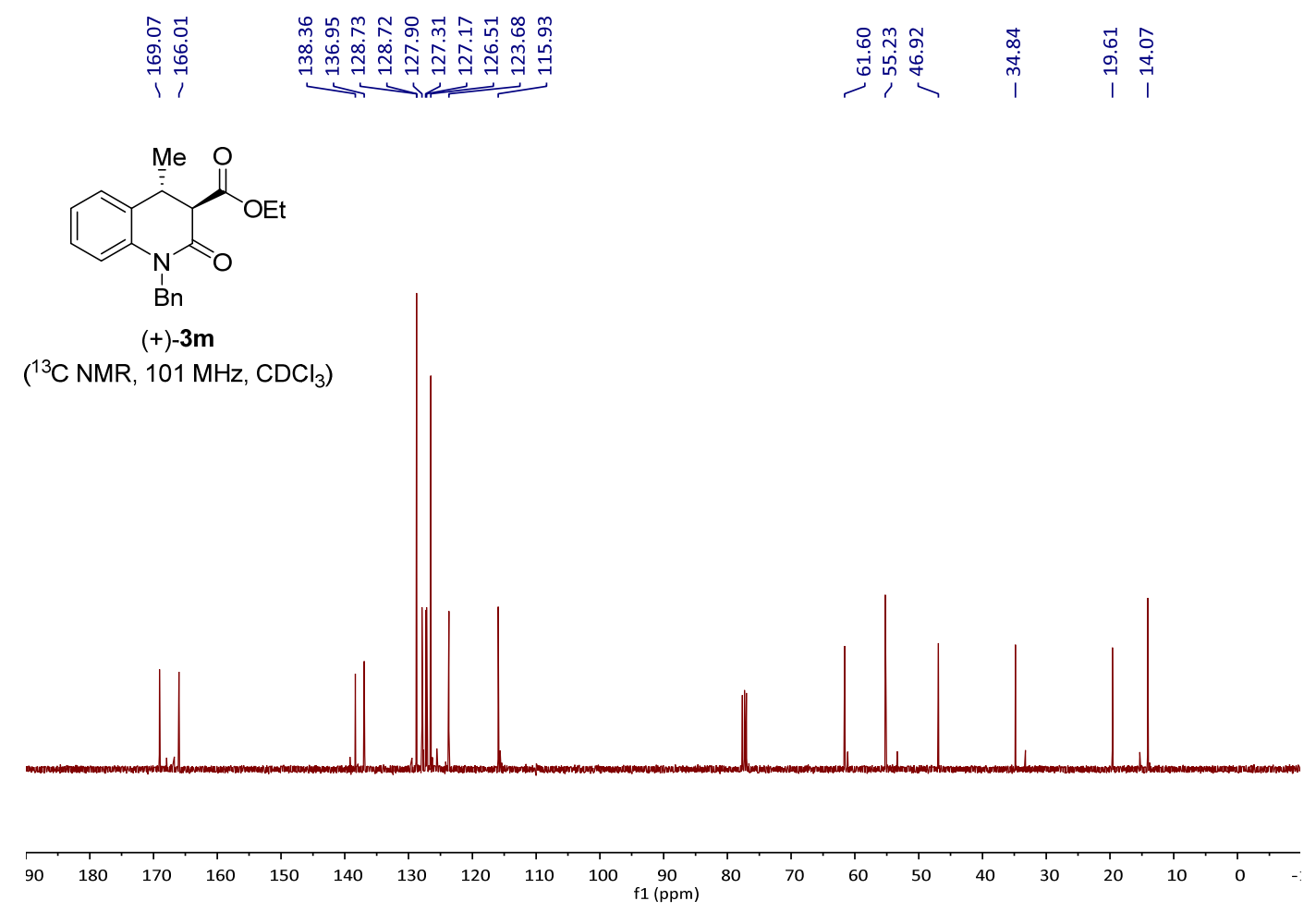

\section{(-)-Ethyl 4-methyl-2-oxo-1-phenyl-1,2,3,4-tetrahydroquinoline-3-carboxylate (3n)}
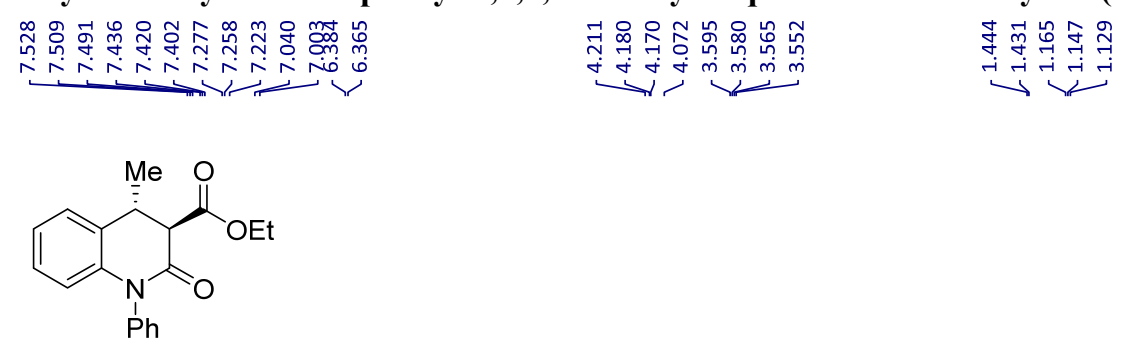

$(-)-3 \mathbf{n}$

$\left({ }^{1} \mathrm{HNMR}, 400 \mathrm{MHz}, \mathrm{CDCl}_{3}\right)$

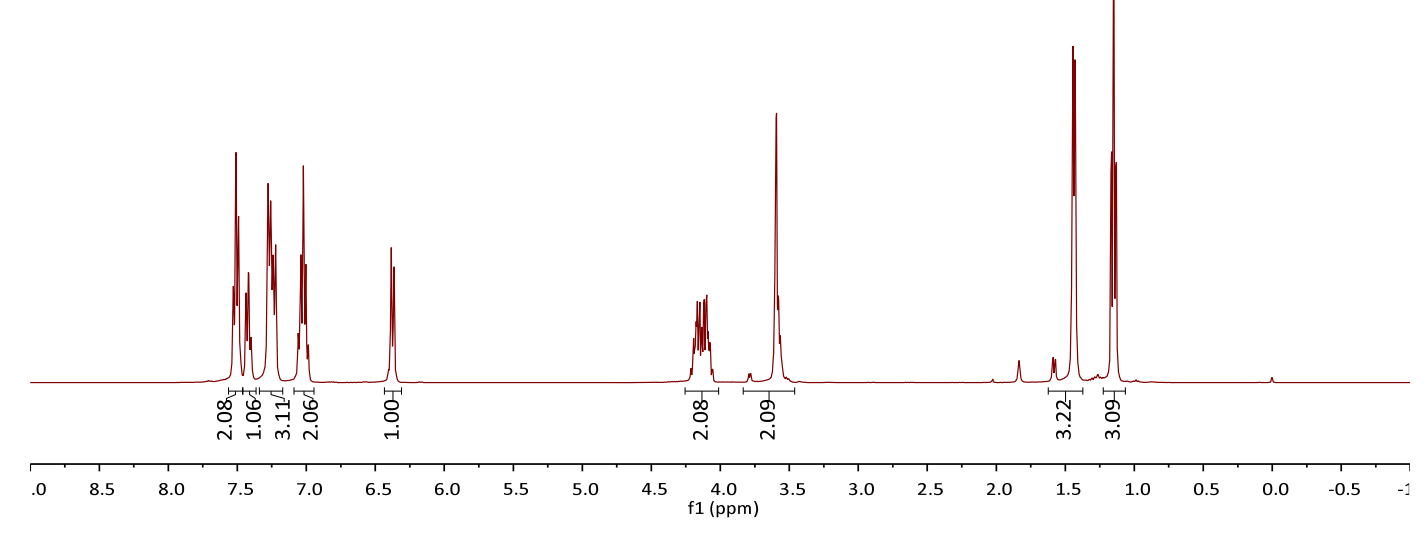


<smiles>CCOC(=O)[C@H]1C(=O)N(c2ccccc2)c2ccccc2[C@@H]1C</smiles>

Ph

$(-)-3 \mathbf{n}$

$\left({ }^{13} \mathrm{C} \mathrm{NMR}, 101 \mathrm{MHz}, \mathrm{CDCl}_{3}\right)$

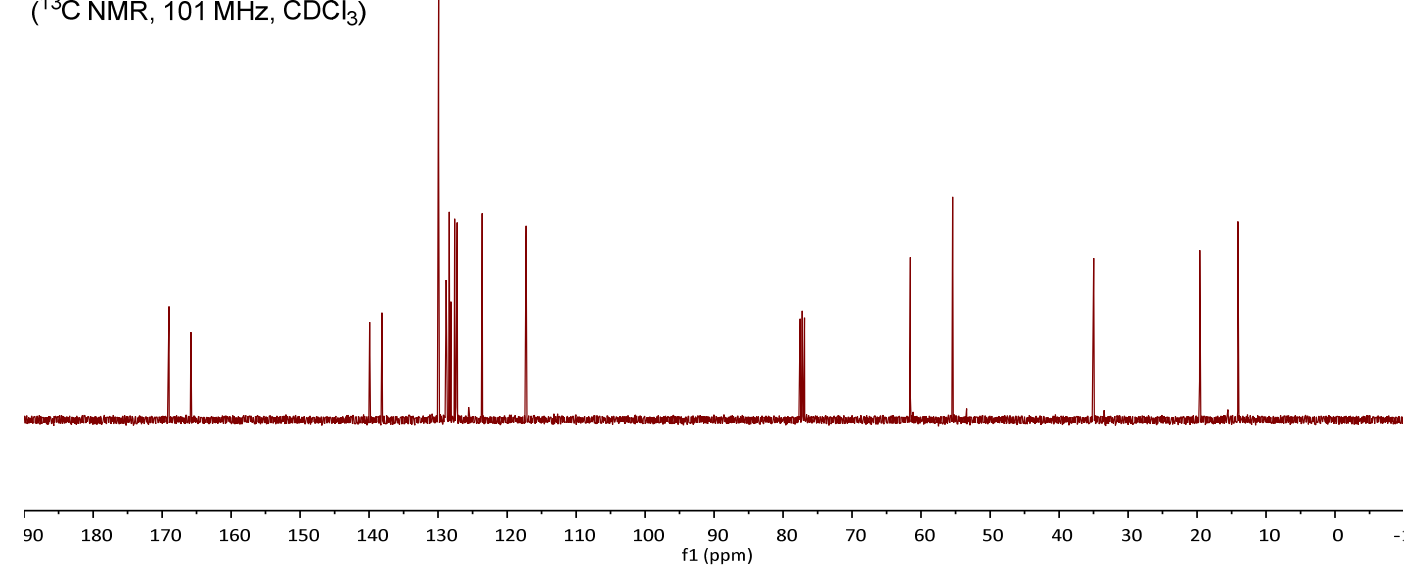

(-)-(3R,4R)-Ethyl 4-ethyl-2-oxo-1-phenyl-1,2,3,4-tetrahydroquinoline-3-carboxylate ((3R,4R)-30)

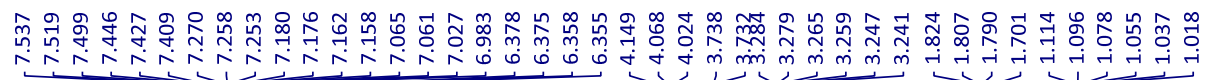<smiles>CCOC(=O)[C@H]1C(=O)N(c2ccccc2)c2ccccc2[C@H]1CC</smiles>

(3R,4R)-30

$\left({ }^{1} \mathrm{HNMR}, 400 \mathrm{MHz}, \mathrm{CDCl}_{3}\right)$

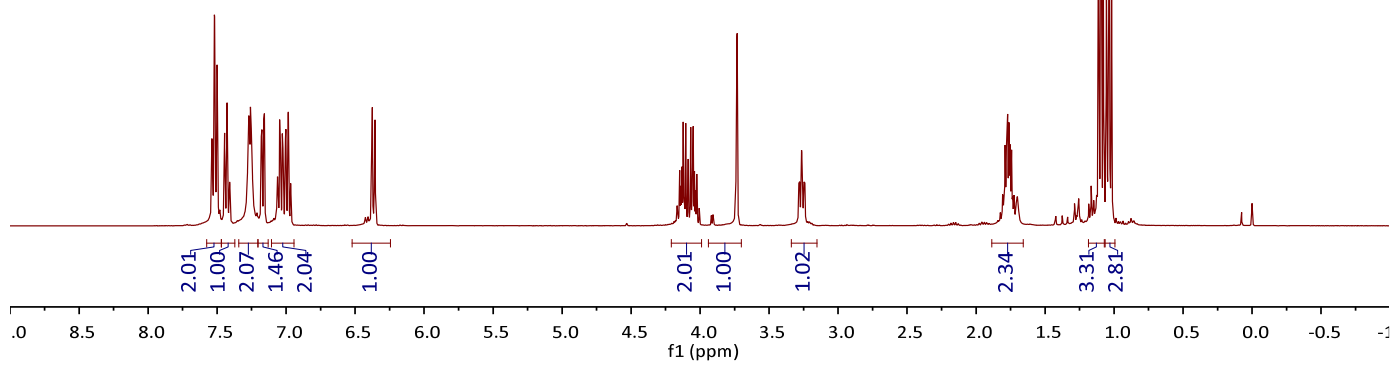




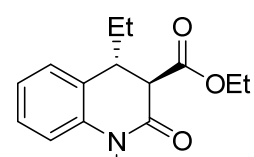

$\mathrm{Ph}$

$(3 R, 4 R)-30$

$\left({ }^{13} \mathrm{C}\right.$ NMR, $\left.101 \mathrm{MHz}, \mathrm{CDCl}_{3}\right)$
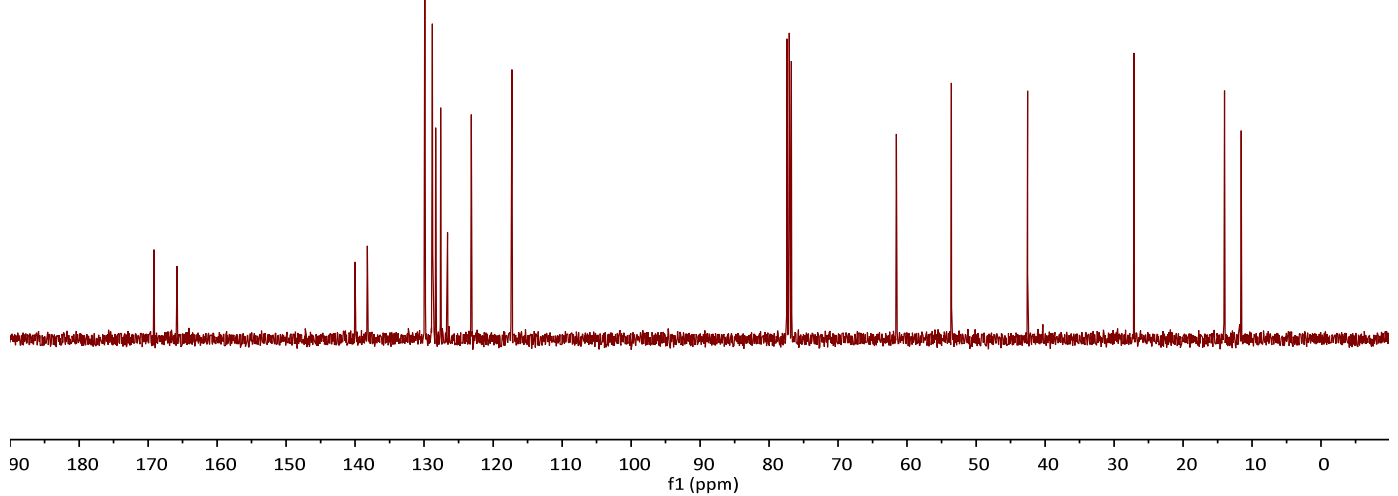

(+)-Ethyl 4-methyl-2-oxo-1,2,3,4-tetrahydroquinoline-3-carboxylate (3p)

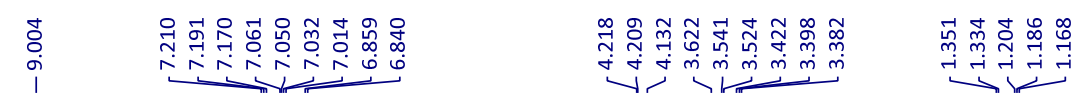<smiles>CCOC(=O)[C@H]1C(=O)Nc2ccccc2[C@@H]1C</smiles>

(+)-3p

$\left({ }^{1} \mathrm{HNMR}, 400 \mathrm{MHz}, \mathrm{CDCl}_{3}\right.$ )

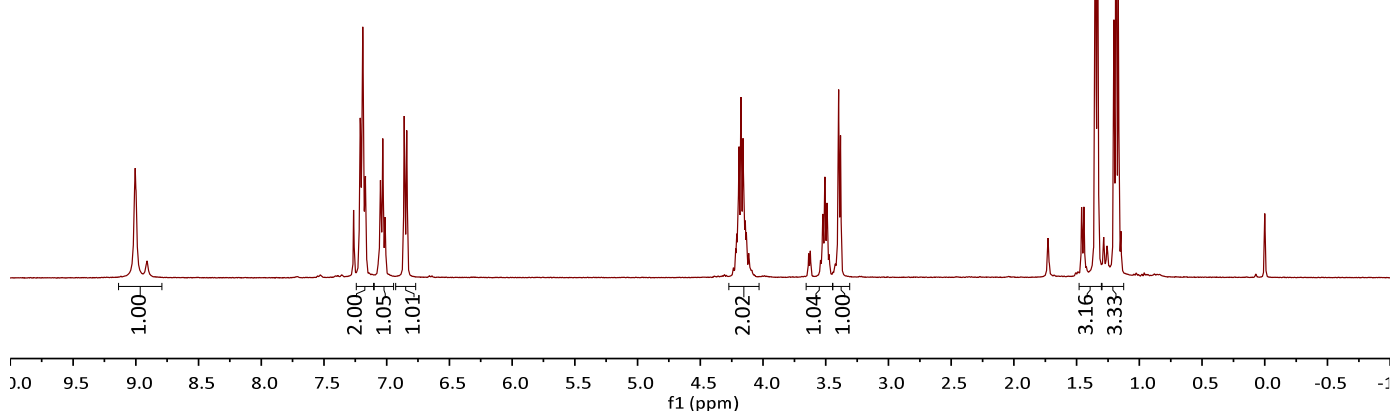




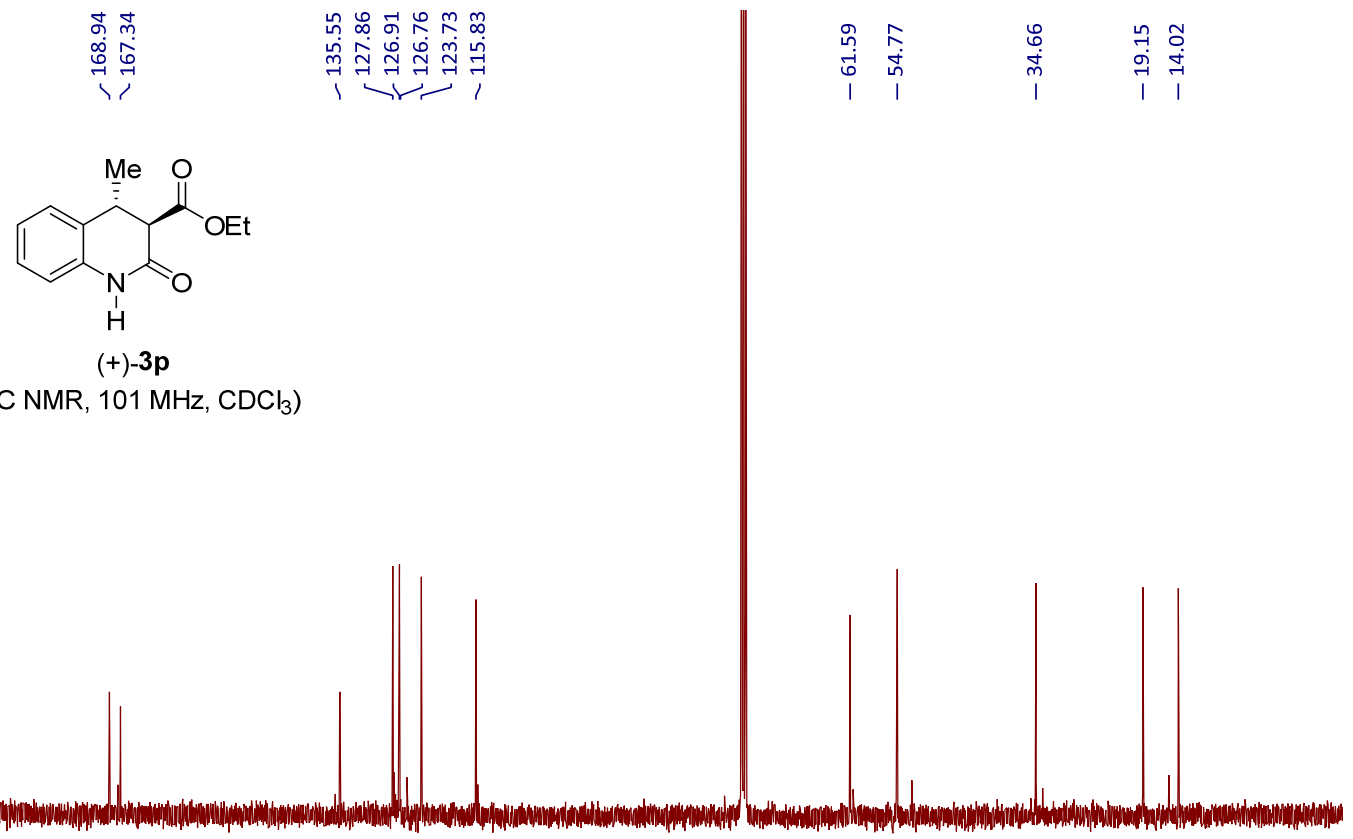

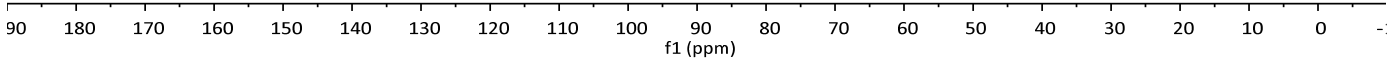

(+)-(3S,4R)-Ethyl-4-methyl-2-oxochromane-3-carboxylate (5a)

$\underbrace{.0}$

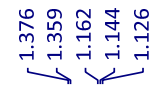<smiles>CCOC(=O)C1C(=O)Oc2ccccc2C1C</smiles>

$(3 S, 4 R)-5 \mathbf{a}$

( ${ }^{1} \mathrm{H} \mathrm{NMR}, 400 \mathrm{MHz}, \mathrm{CDCl}_{3}$ )

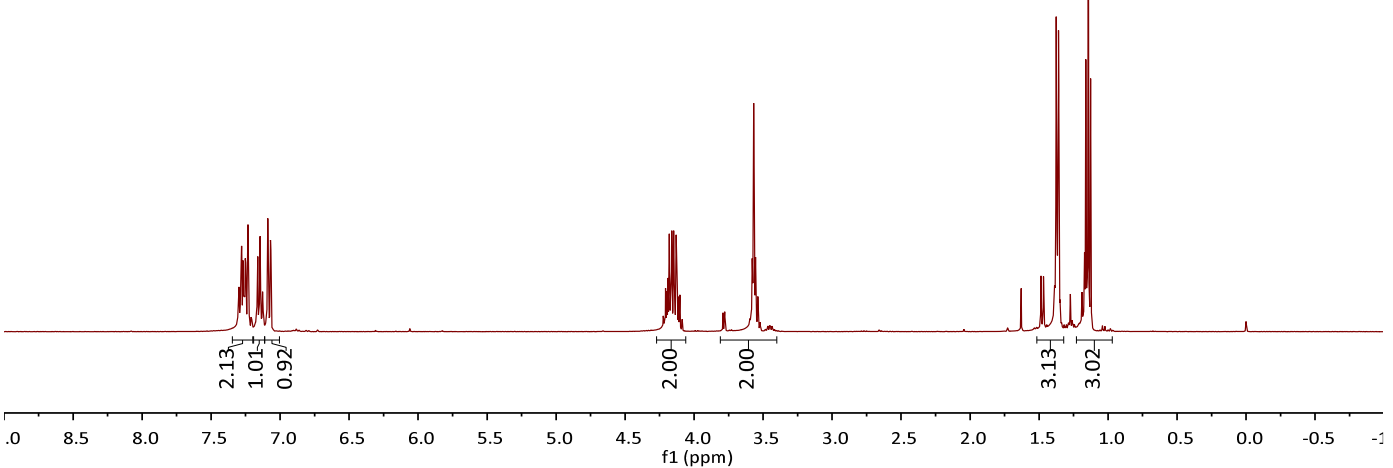




\section{(+)-(3S,4R)-Ethyl-4-ethyl-2-oxochromane-3-carboxylate (5b)}

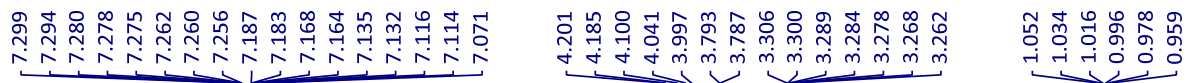<smiles>CCOC(=O)[C@H]1C(=O)Oc2ccccc2C1CC</smiles>

$(3 S, 4 R)-5 b$

( ${ }^{1} \mathrm{H} \mathrm{NMR}, 400 \mathrm{MHz}, \mathrm{CDCl}_{3}$ )

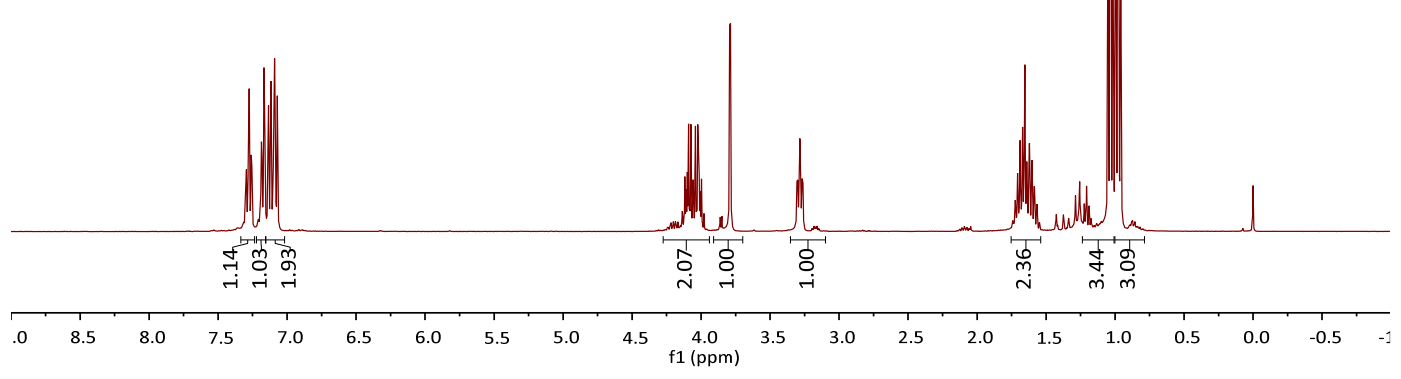

(+)-Ethyl 2-oxo-4-propylchromane-3-carboxylate (5c)

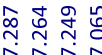

声

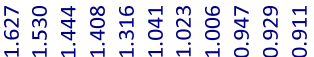

rivin<smiles>CCOC(=O)C1C(=O)Oc2ccccc2C1C(C)C</smiles>

$(+)-5 \mathrm{c}$

( ${ }^{1} \mathrm{H} \mathrm{NMR}, 400 \mathrm{MHz}, \mathrm{CDCl}_{3}$ )

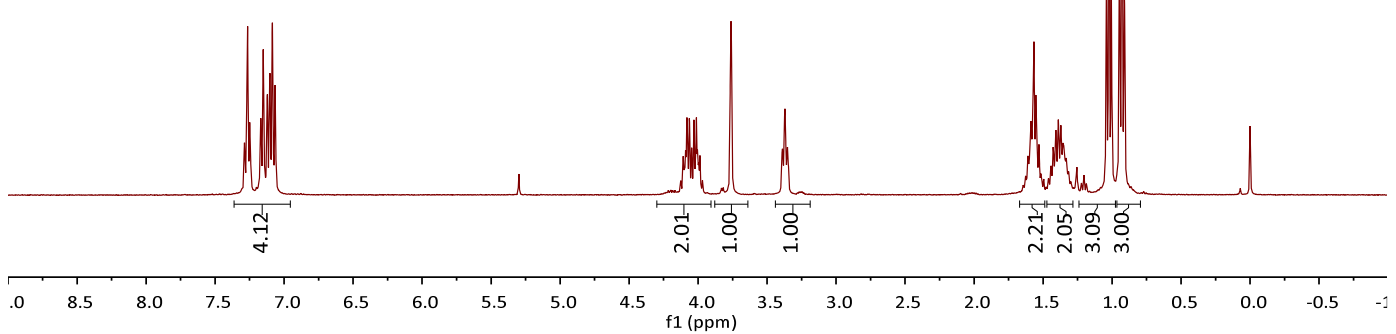




\section{(+)-Ethyl-4-isobutyl-2-oxochromane-3-carboxylate (5d)}

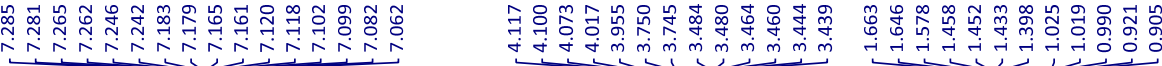<smiles>CCOC(=O)C1Cc2ccccc2OC1=O</smiles>

$(+)-5 d$

$\left({ }^{1} \mathrm{H} \mathrm{NMR}, 400 \mathrm{MHz}, \mathrm{CDCl}_{3}\right)$

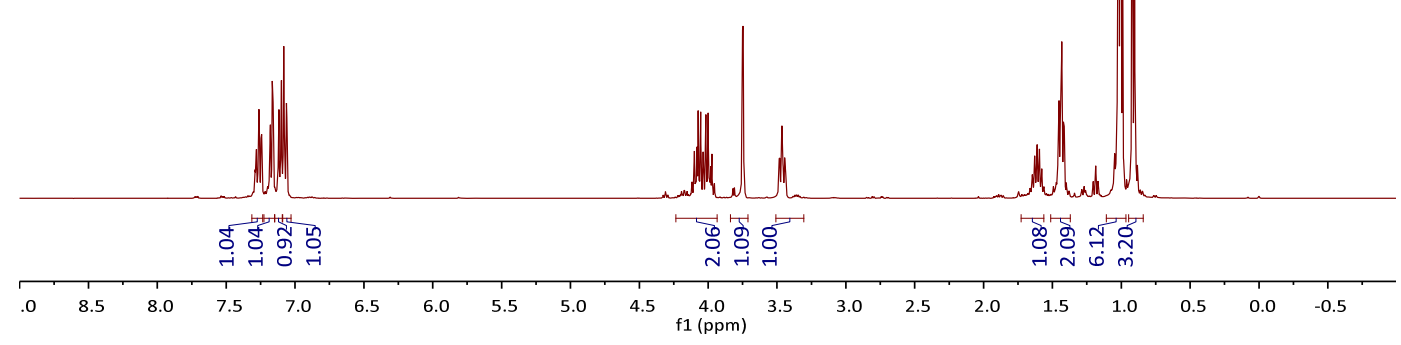

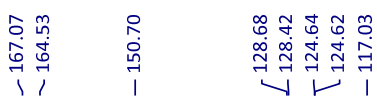

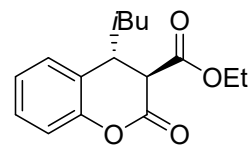

$(+)-5 d$

$\left({ }^{13} \mathrm{C} \mathrm{NMR}, 101 \mathrm{MHz}, \mathrm{CDCl}_{3}\right)$

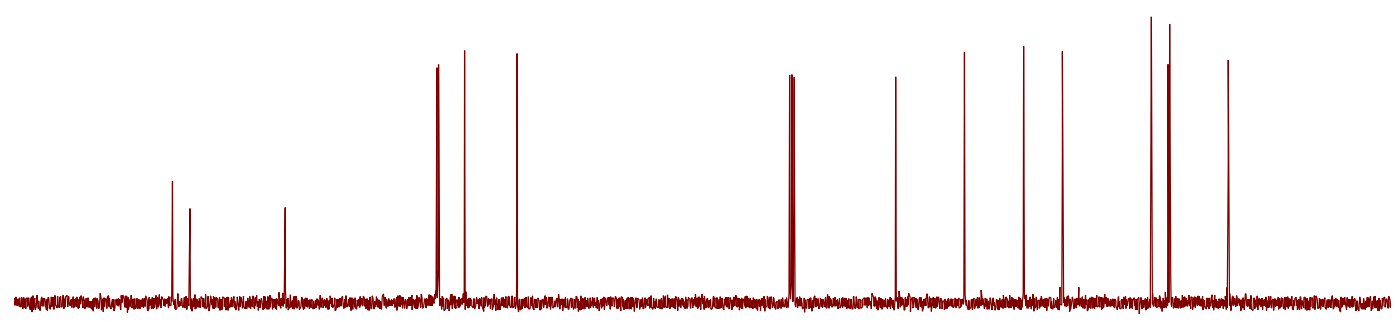

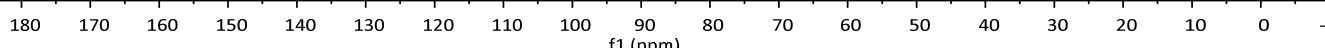




\section{(-)-Ethyl-4-isopropyl-2-oxochromane-3-carboxylate (5e)}

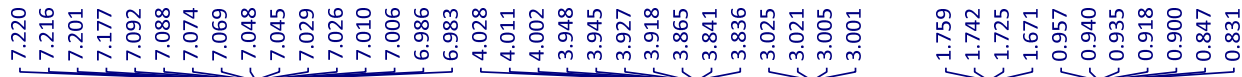<smiles>CCOC(=O)C1C(=O)Oc2ccccc2C1C(C)C</smiles>

$(-)-5 e$

$\left({ }^{1} \mathrm{H} \mathrm{NMR}, 400 \mathrm{MHz}, \mathrm{CDCl}_{3}\right)$

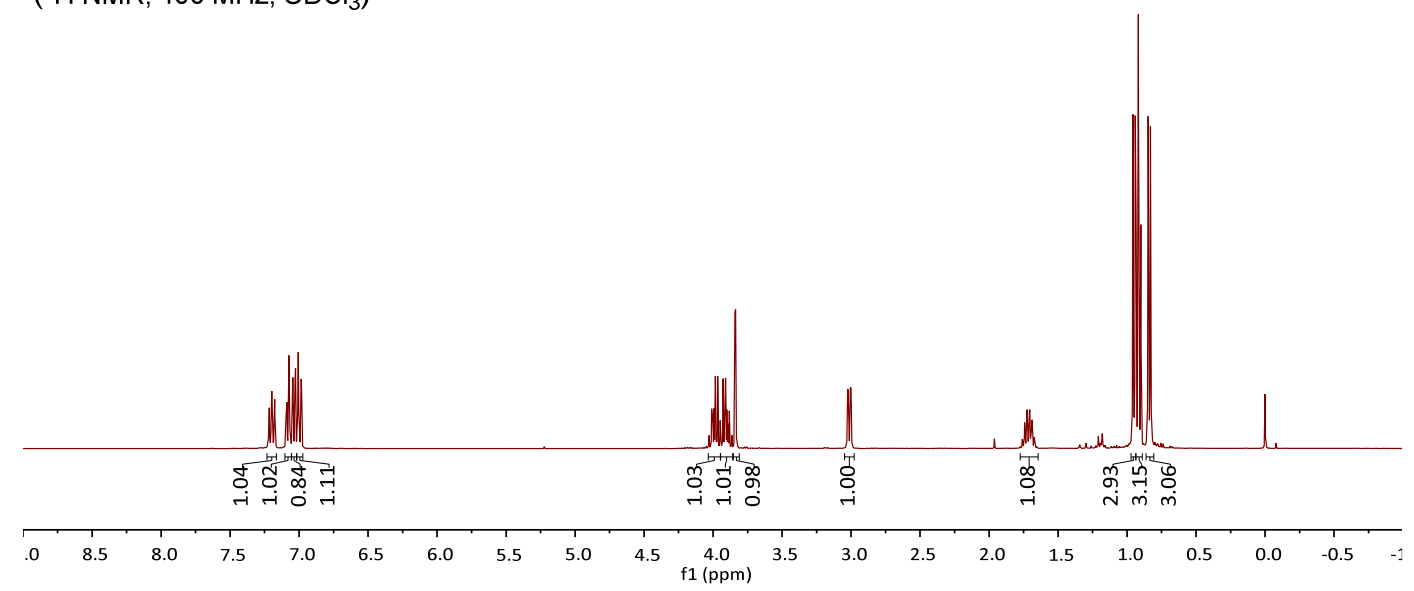

(-)-Ethyl-4-cyclohexyl-2-oxochromane-3-carboxylate (5f)

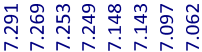

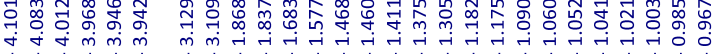

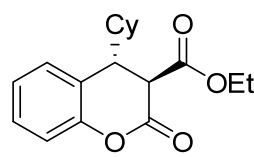

$(-)-5 f$

$\left({ }^{1} \mathrm{H}\right.$ NMR, $\left.400 \mathrm{MHz}, \mathrm{CDCl}_{3}\right)$

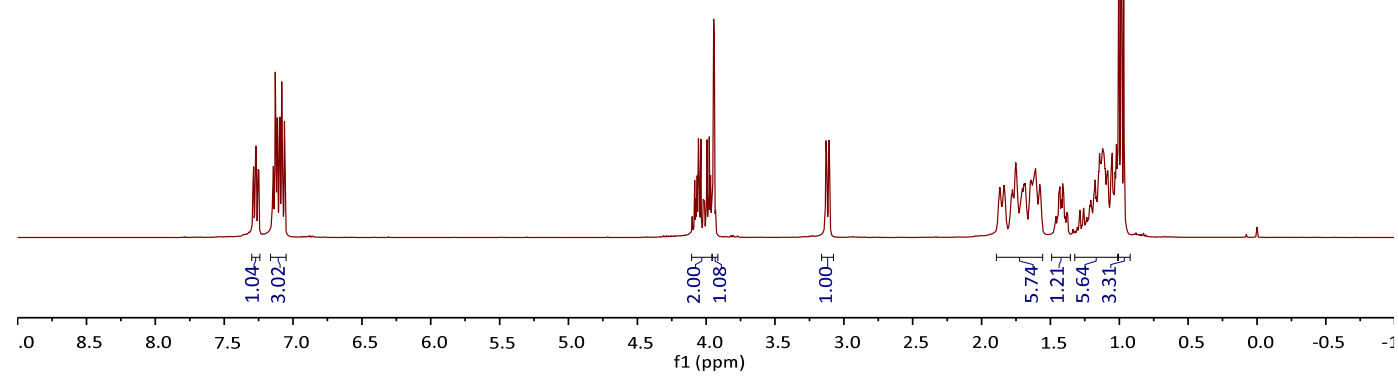


<smiles>CCOC(=O)C1Cc2ccccc2OC1=O</smiles>

$(-)-5 f$

$\left({ }^{13} \mathrm{C} \mathrm{NMR}, 101 \mathrm{MHz}, \mathrm{CDCl}_{3}\right)$

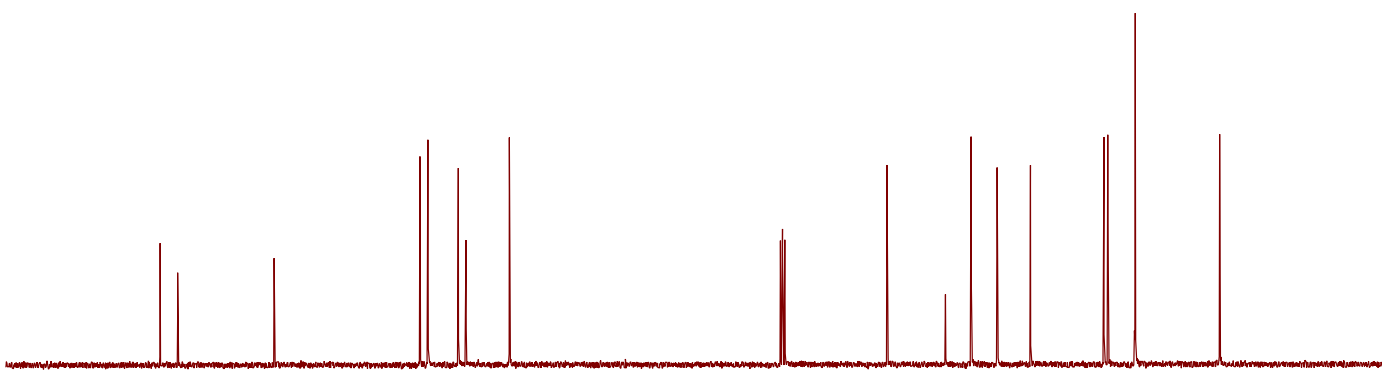

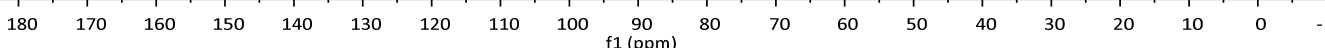

(+)-Ethyl-4-benzyl-2-oxochromane-3-carboxylate (5g)

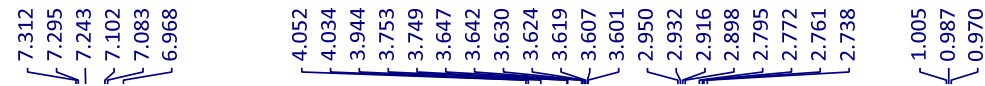<smiles>CCOC(=O)[C@H]1C(=O)Oc2ccccc2[C@H]1Cc1ccccc1</smiles>

$(+)-5 \mathbf{g}$

$\left({ }^{1} \mathrm{H} \mathrm{NMR}, 400 \mathrm{MHz}, \mathrm{CDCl}_{3}\right)$

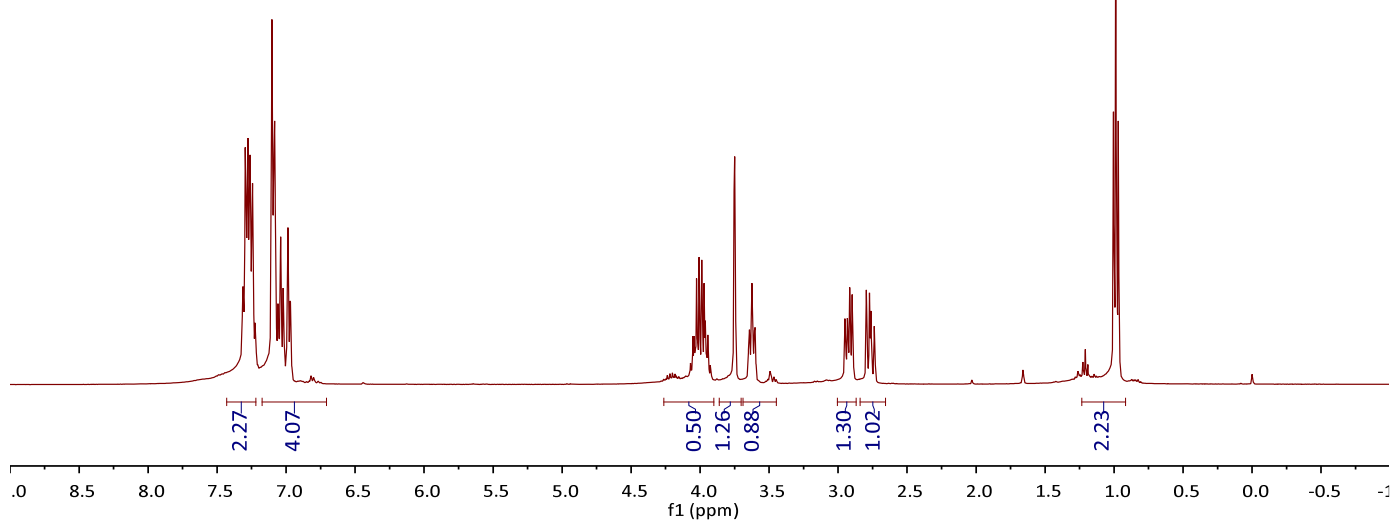


(+)-Ethyl-2-oxo-4-phenethylchromane-3-carboxylate (5h)

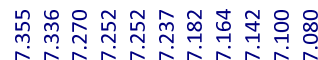

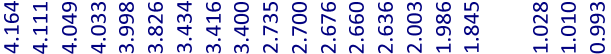

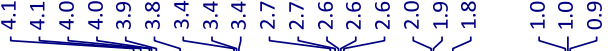<smiles>CCOC(=O)[C@H]1C(=O)Oc2ccccc2[C@H]1CCc1ccccc1</smiles>

$(+)-5 \mathrm{~h}$

$\left({ }^{1} \mathrm{H} \mathrm{NMR}, 400 \mathrm{MHz}, \mathrm{CDCl}_{3}\right)$

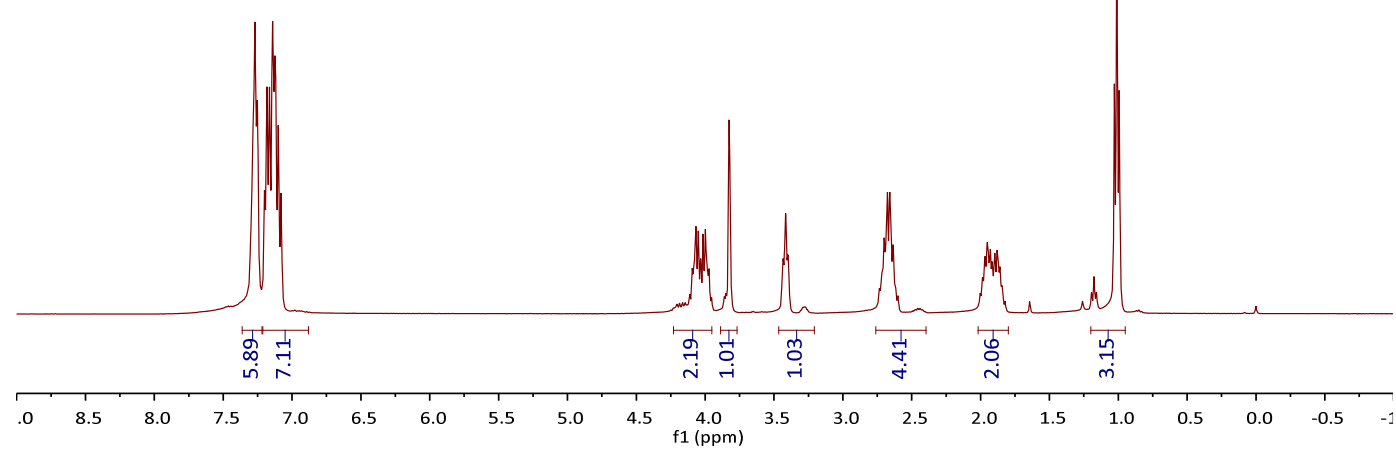

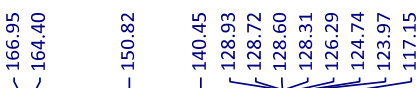

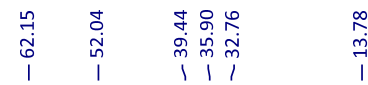<smiles>CCOC(=O)[C@H]1C(=O)Oc2ccccc2[C@H]1CCc1ccccc1</smiles>

$(+)-5 \mathrm{~h}$

$\left({ }^{13} \mathrm{C}\right.$ NMR, $\left.101 \mathrm{MHz}, \mathrm{CDCl}_{3}\right)$

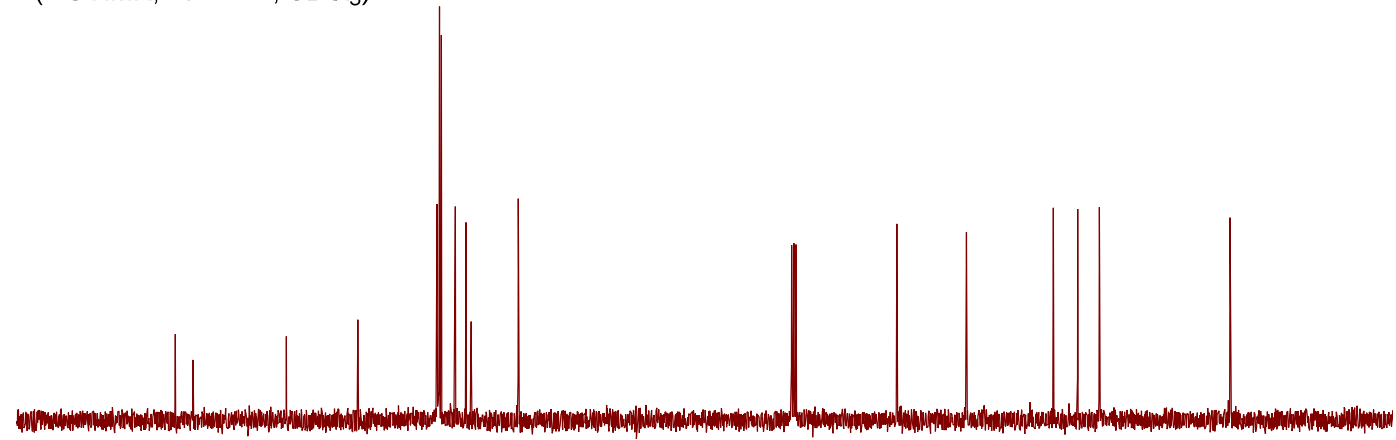

$\begin{array}{llllllllllllllllllll}90 & 180 & 170 & 160 & 150 & 140 & 130 & 120 & 110 & 100 & 90 & 80 & 70 & 60 & 50 & 40 & 30 & 20 & 10 & 0\end{array}$ 
(+)-Ethyl-4-(4-ethoxy-4-oxobutyl)-2-oxochromane-3-carboxylate (5i)

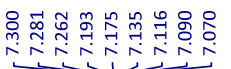

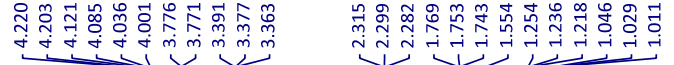<smiles>CCOCCC1c2ccccc2OC(=O)C1C(=O)OCC</smiles>

$(+)-5 \mathbf{i}$

( ${ }^{1} \mathrm{H} \mathrm{NMR}, 400 \mathrm{MHz}, \mathrm{CDCl}_{3}$ )

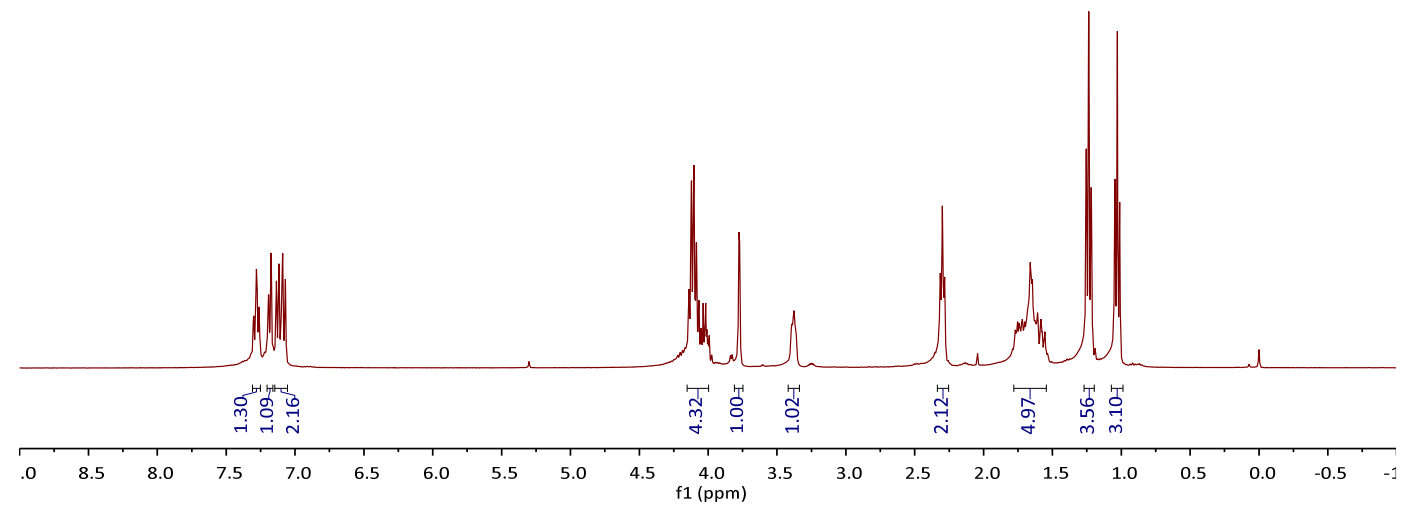

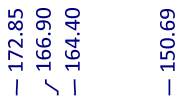

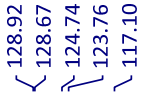

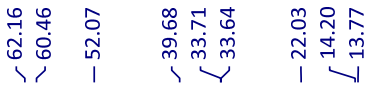

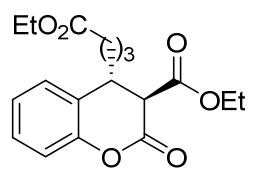

$(+)-5 \mathbf{i}$

$\left({ }^{13} \mathrm{C} \mathrm{NMR}, 101 \mathrm{MHz}, \mathrm{CDCl}_{3}\right)$

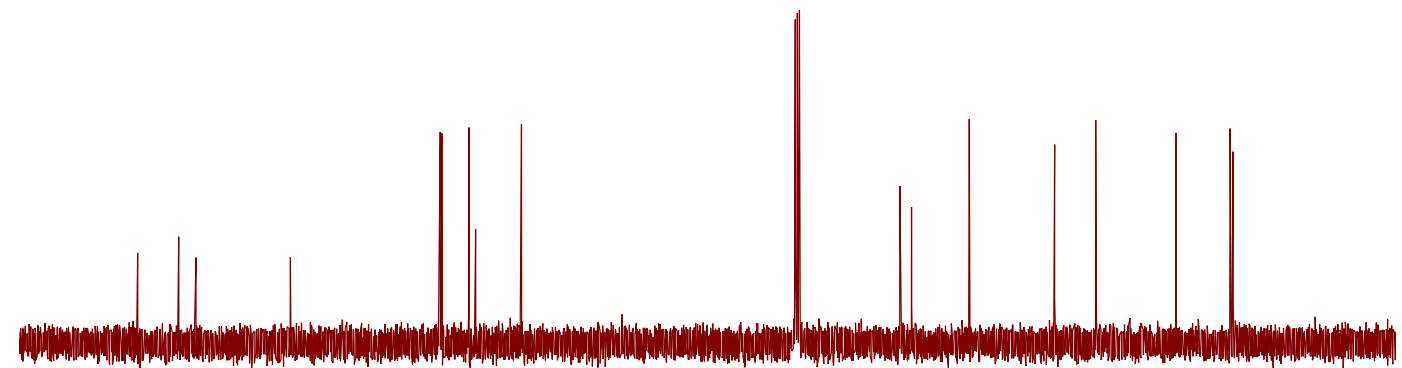

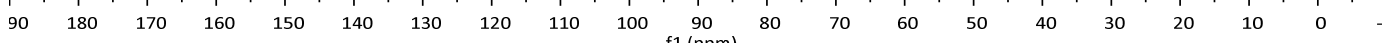


(+)-Ethyl-4-(2-((tert-butoxycarbonyl)(methyl)amino)ethyl)-2-oxochromane-3-carboxylate (5j)

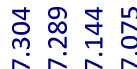

inctir

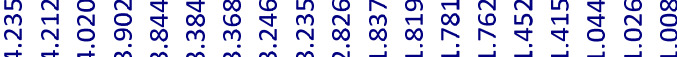<smiles>CCOC(=O)[C@H]1C(=O)Oc2ccccc2[C@H]1CN(C)C(=O)OCc1ccccc1</smiles>

$(+)-5 j$

$\left({ }^{1} \mathrm{H} \mathrm{NMR}, 400 \mathrm{MHz}, \mathrm{CDCl}_{3}\right)$

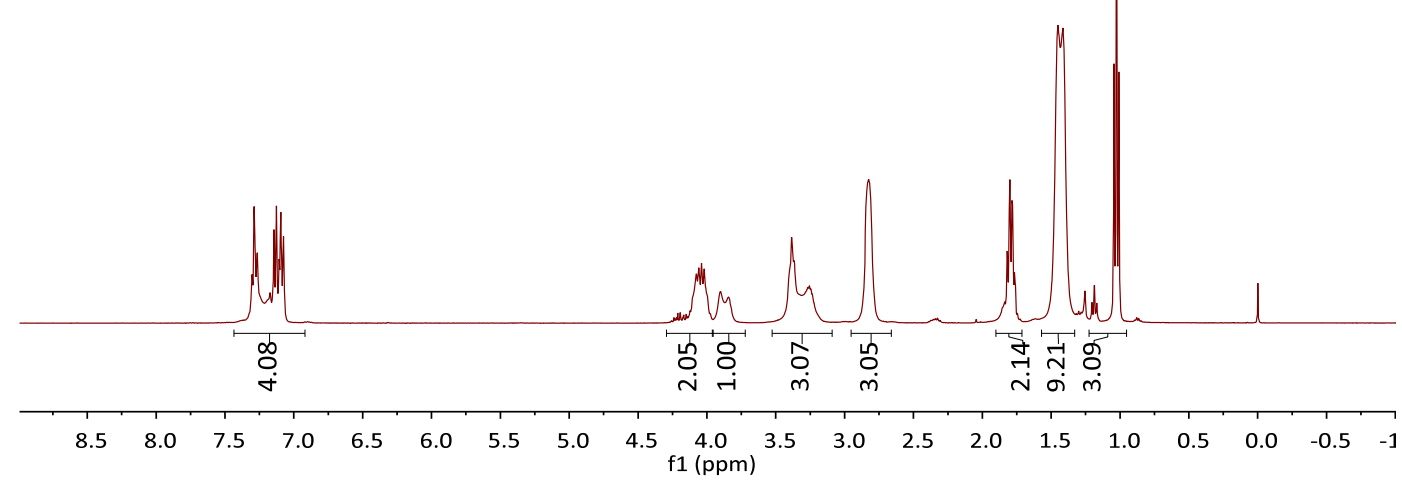

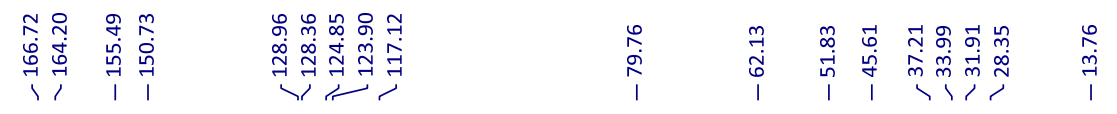

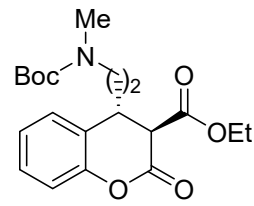

$(+)-5$ j

$\left({ }^{13} \mathrm{C} \mathrm{NMR}, 101 \mathrm{MHz}, \mathrm{CDCl}_{3}\right)$

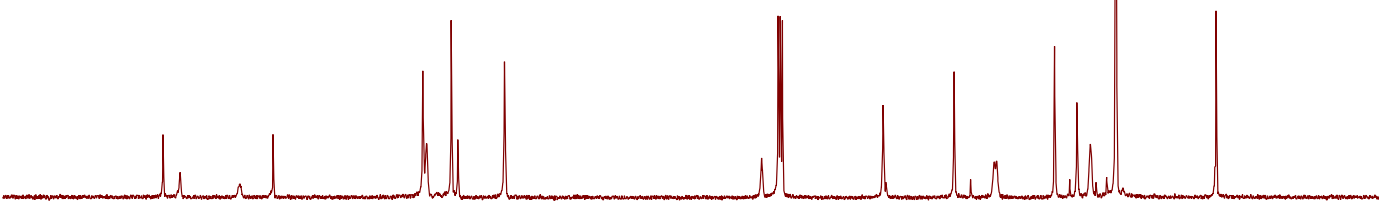

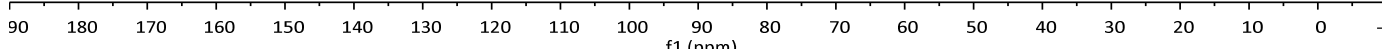


$(+)-(3 S, 4 R)$-Ethyl-7-methoxy-2-oxo-4-pentylchromane-3-carboxylate ((3S,4R)-5k)

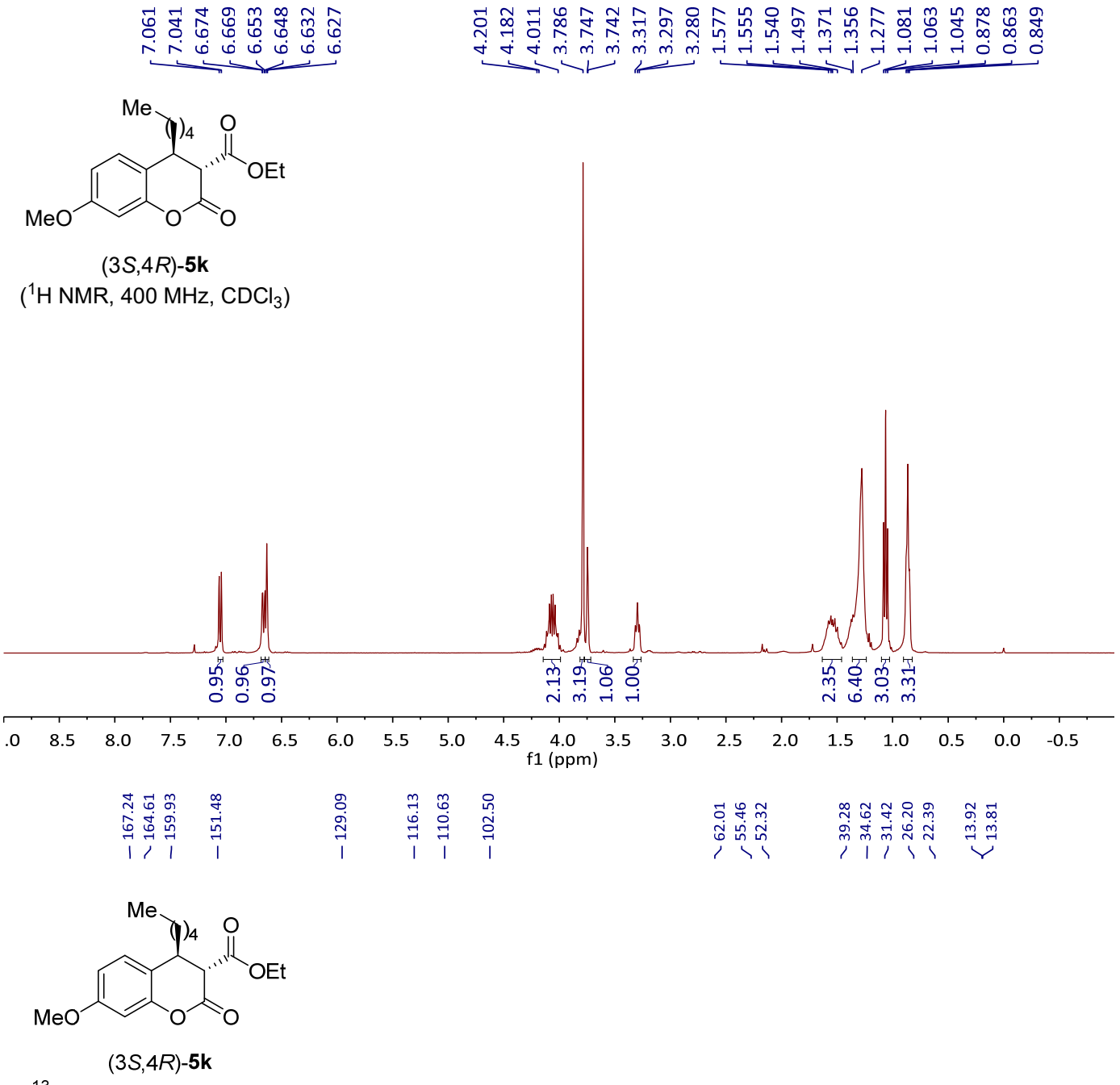

$\left({ }^{13} \mathrm{C} \mathrm{NMR}, 101 \mathrm{MHz}, \mathrm{CDCl}_{3}\right)$

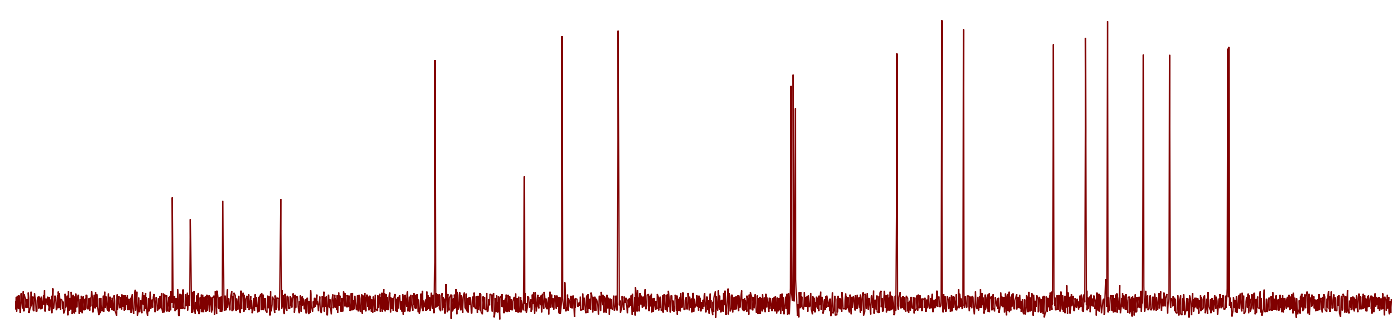

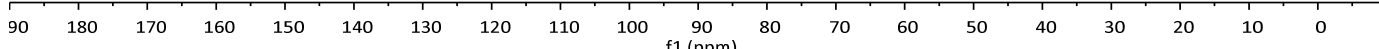


(+)-Ethyl-4-cyclopropyl-2-oxochromane-3-carboxylate (5l)

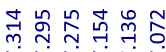

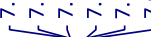

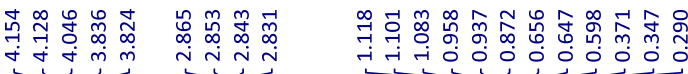<smiles>CCOC(=O)C1C(=O)Oc2ccccc2C1C1CC1</smiles>

$(+)-51$

$\left({ }^{1} \mathrm{H}\right.$ NMR, $400 \mathrm{MHz}, \mathrm{CDCl}_{3}$ )

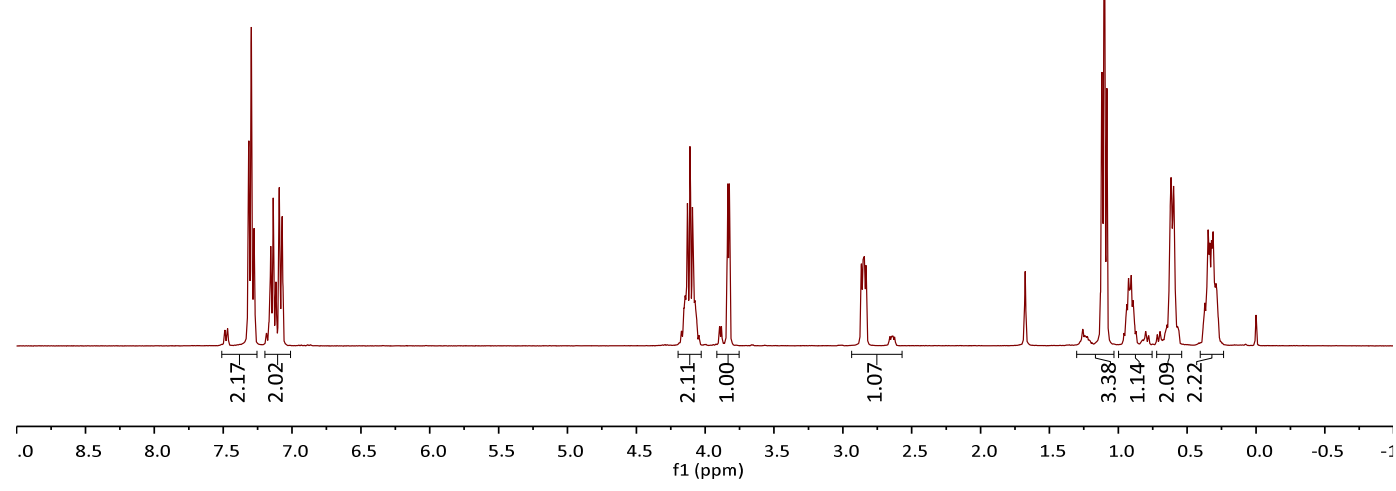

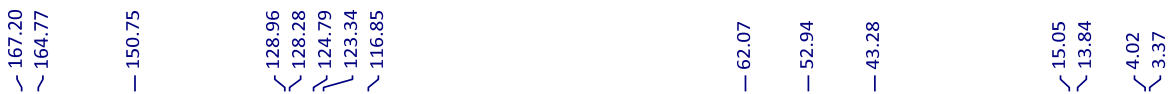

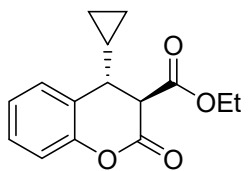

$(+)-5$ I

$\left({ }^{13} \mathrm{C} \mathrm{NMR}, 101 \mathrm{MHz}, \mathrm{CDCl}_{3}\right)$

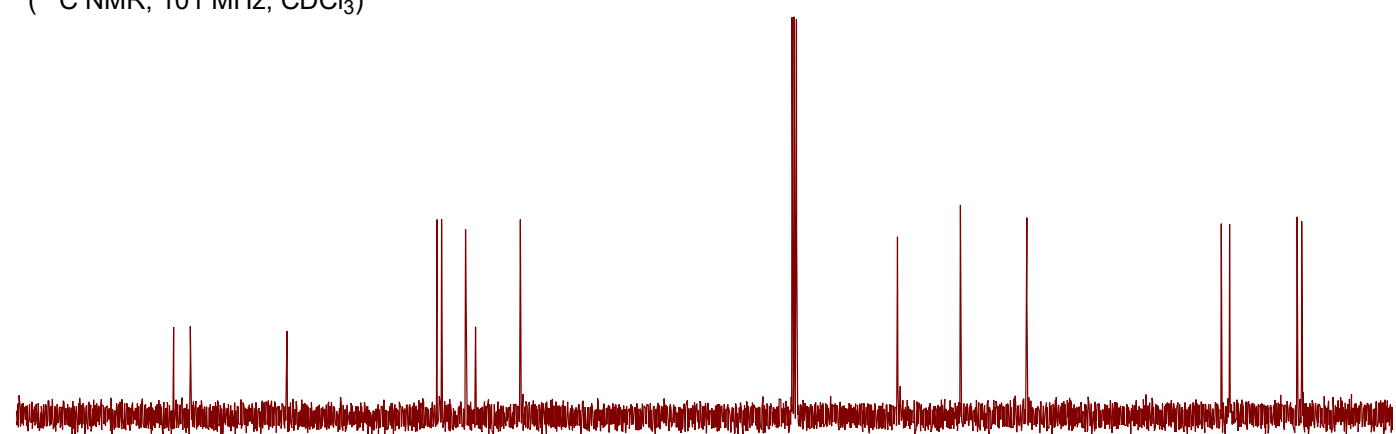

$\begin{array}{llllllllllllllllllll}90 & 180 & 170 & 160 & 150 & 140 & 130 & 120 & 110 & 100 & 90 & 80 & 70 & 60 & 50 & 40 & 30 & 20 & 10 & 0\end{array}$ 
(+)-Ethyl-4-cyclopropyl-7-methoxy-2-oxochromane-3-carboxylate (5m)

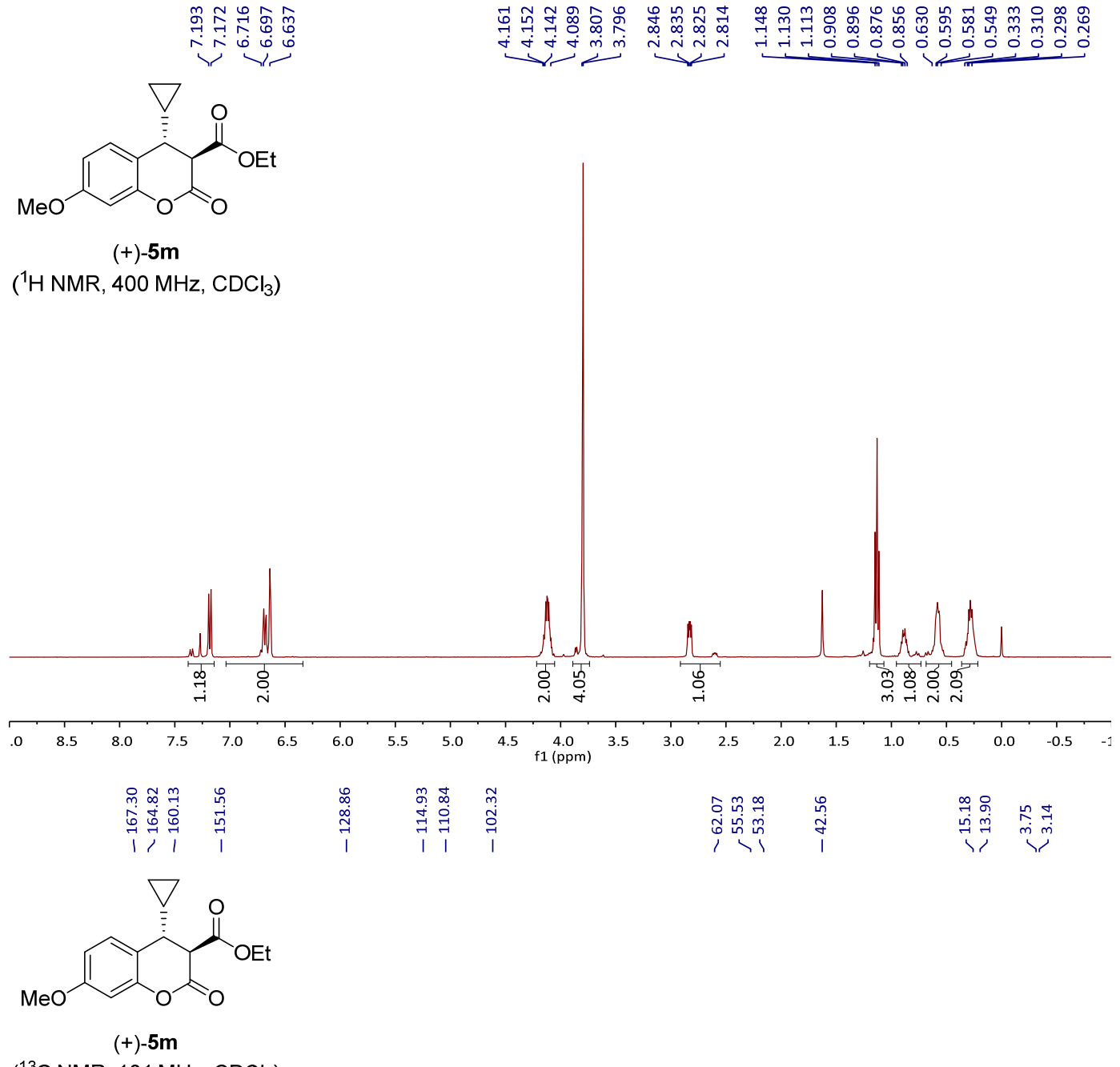

$\left({ }^{13} \mathrm{C} \mathrm{NMR}, 101 \mathrm{MHz}, \mathrm{CDCl}_{3}\right)$

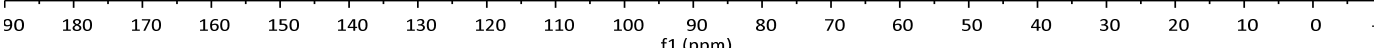


(+)-Ethyl-4-cyclopropyl-6-methoxy-2-oxochromane-3-carboxylate (5n)

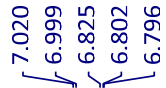

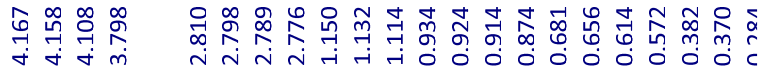<smiles>CCOC(=O)C1C(=O)Oc2ccc(OC)cc2C1C1CC1</smiles>

$(+)-5 n$

$\left({ }^{1} \mathrm{H} \mathrm{NMR}, 400 \mathrm{MHz}, \mathrm{CDCl}_{3}\right)$

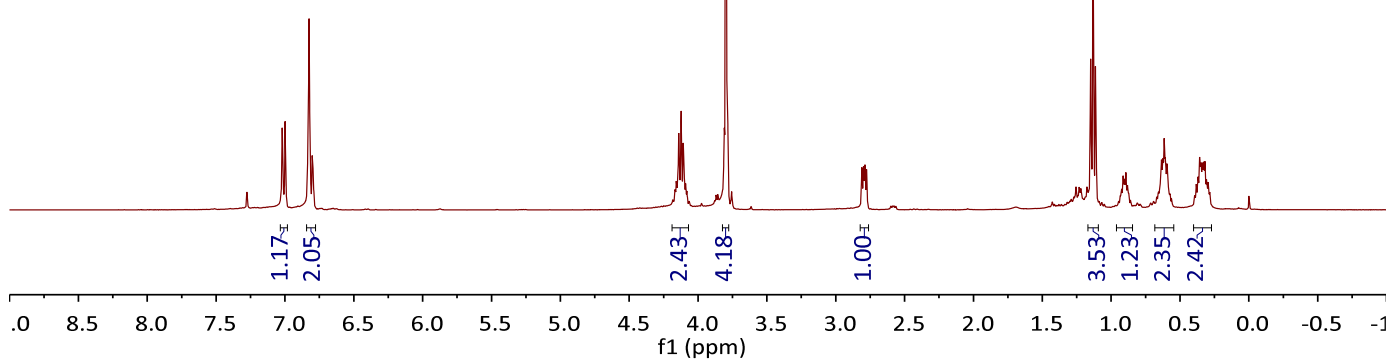

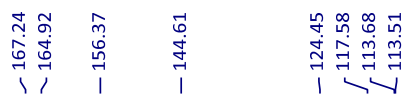

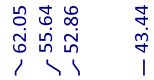

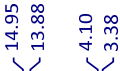<smiles>CCOC(=O)C1C(=O)Oc2ccc(OC)cc2C1C1CC1</smiles>

$(+)-5 n$

$\left({ }^{13} \mathrm{C} \mathrm{NMR}, 101 \mathrm{MHz}, \mathrm{CDCl}_{3}\right)$

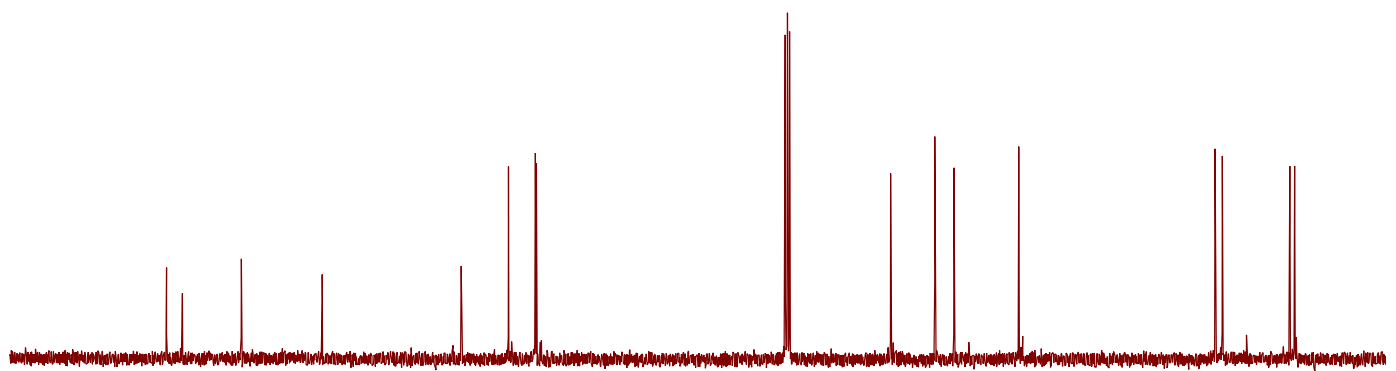

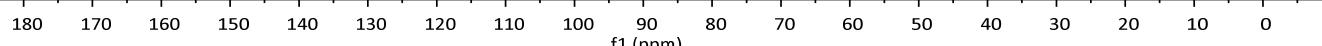


(-)-(3R,4R)-Ethyl-2-oxo-4-phenylchromane-3-carboxylate (5o)

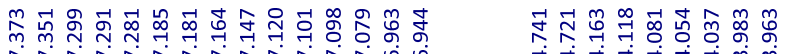

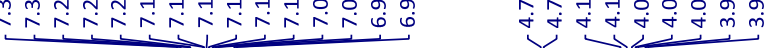<smiles>CCOC(=O)[C@H]1C(=O)Oc2ccccc2[C@@H]1c1ccccc1</smiles>

(-)-50

$\left({ }^{1} \mathrm{H}\right.$ NMR, $\left.400 \mathrm{MHz}, \mathrm{CDCl}_{3}\right)$

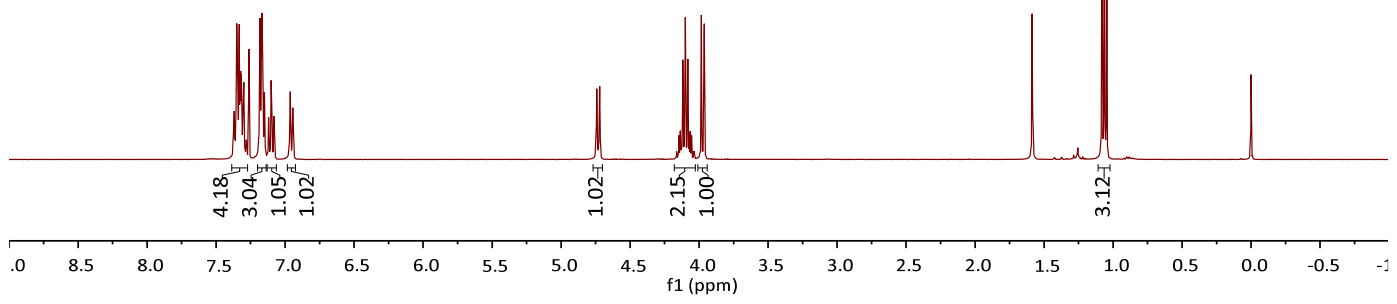

(-)-(S)-7-methoxy-4-pentylchroman-2-one ((S)-6)

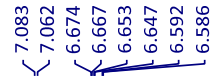

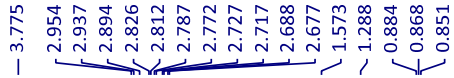<smiles>CCC1CC(=O)Oc2cc(OC)ccc21</smiles>

(S)-6

( ${ }^{1} \mathrm{H}$ NMR, $400 \mathrm{MHz}, \mathrm{CDCl}_{3}$ )

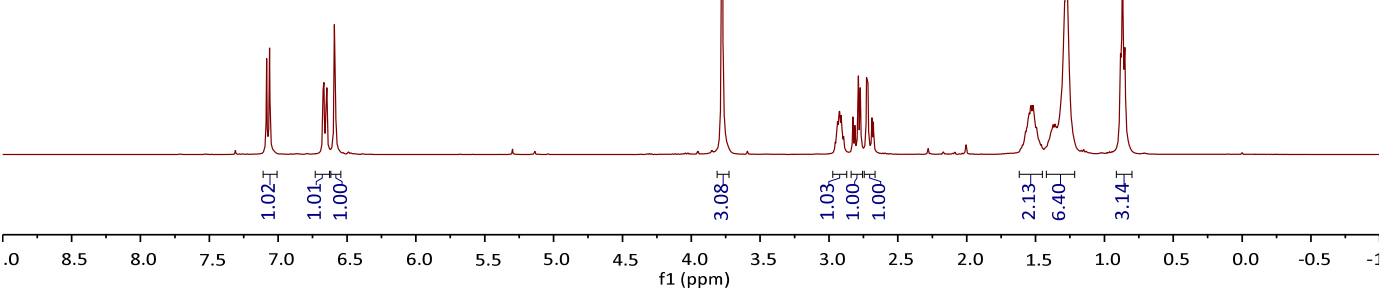




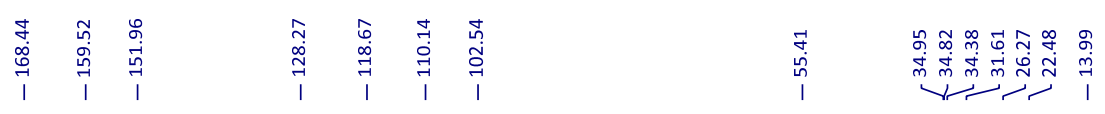<smiles>C=CC1CC(=O)Oc2cc(OC)ccc21</smiles>

(S)-6

$\left({ }^{13} \mathrm{C} \mathrm{NMR,}, 101 \mathrm{MHz}, \mathrm{CDCl}_{3}\right)$

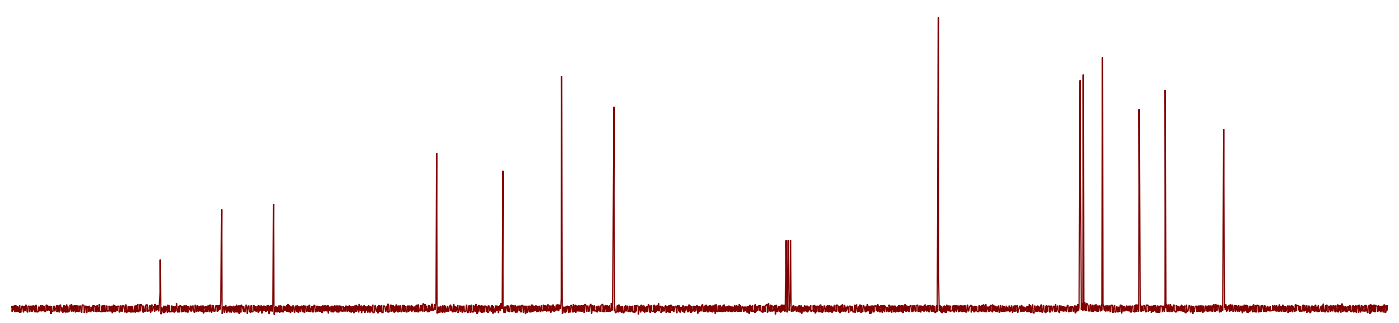

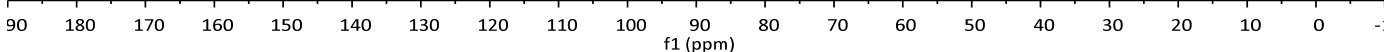

(+)-(S)-3-(2,4-dimethoxyphenyl)octanoic acid ((S)-7)

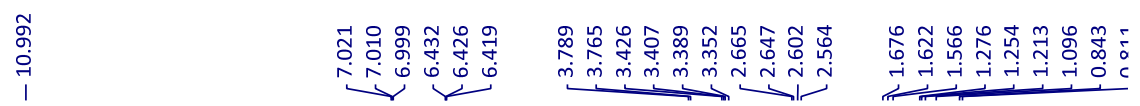<smiles>CCC(CC(=O)O)c1ccc(OC)cc1OC</smiles>

(S)-7

$\left({ }^{1} \mathrm{H}\right.$ NMR, $400 \mathrm{MHz}, \mathrm{CDCl}_{3}$ )

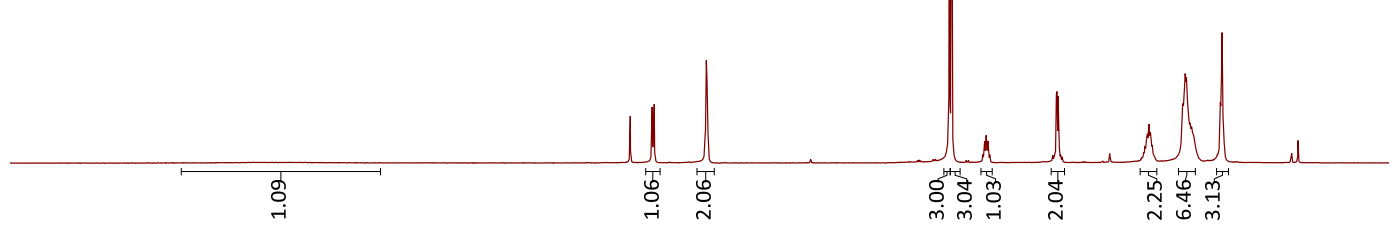

$\begin{array}{llllllllllllllllllllllllllllllllllllllllllllll}13.5 & 13.0 & 12.5 & 12.0 & 11.5 & 11.0 & 10.5 & 1 & 10.0 & 9.5 & 9.0 & 8.5 & 8.0 & 7.5 & 7.0 & 6.5 & 6.0 & 5.5 & 5.0 & 4.5 & 4.0 & 3.5 & 3.0 & 2.5 & 2.0 & 1.5 & 1.0 & 0.5 & 0.0 & -0.5 & -1\end{array}$ 
(+)-(3S,4S)-4-Ethyl-3-methyl-2-oxo-1-phenyl-1,2,3,4-tetrahydroquinoline-3-carboxylic acid $((3 S, 4 S)-8)$

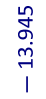

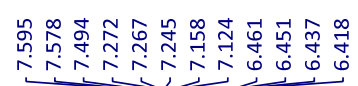

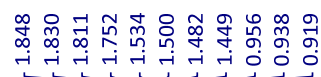

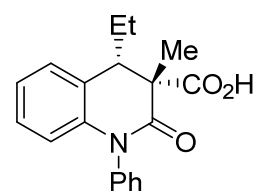

$(3 S, 4 S)-8$

$\left({ }^{1} \mathrm{H} N M R, 400 \mathrm{MHz}, \mathrm{CDCl}_{3}\right)$

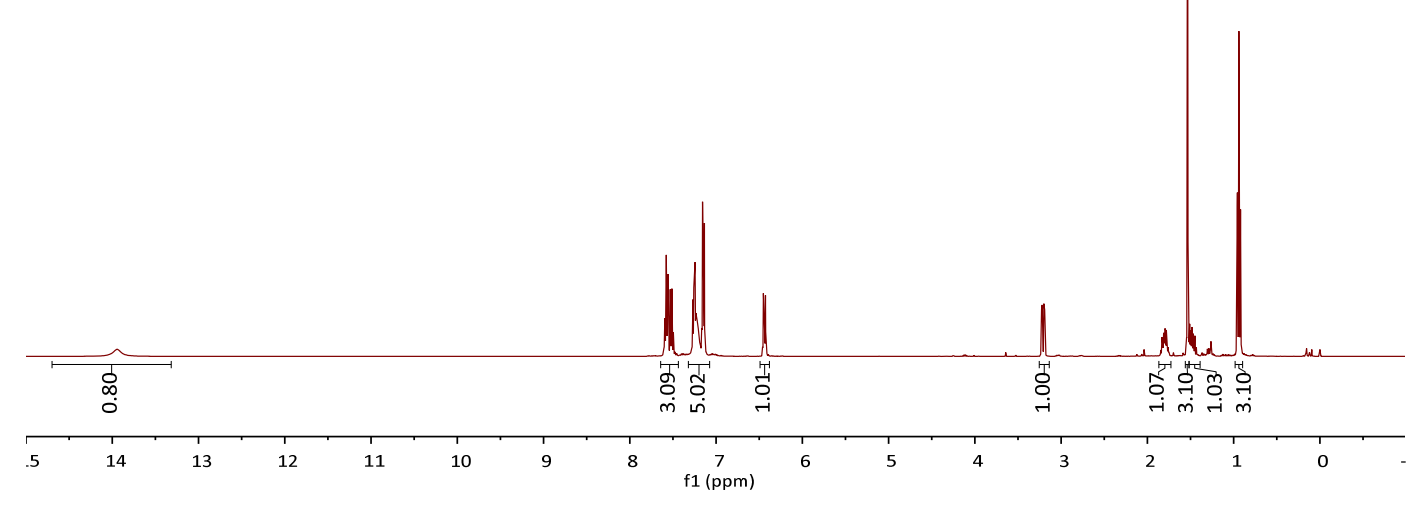

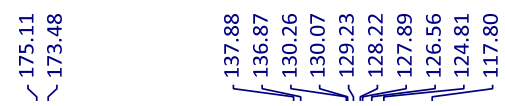

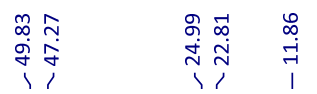

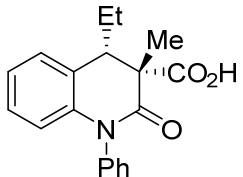

$(3 S, 4 S)-8$

$\left({ }^{13} \mathrm{C} \mathrm{NMR}, 101 \mathrm{MHz}, \mathrm{CDCl}_{3}\right)$

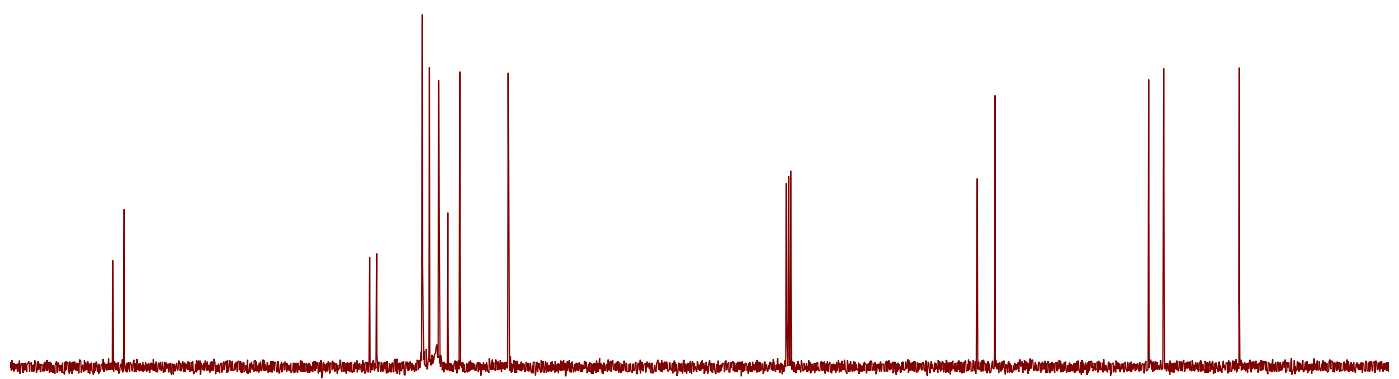

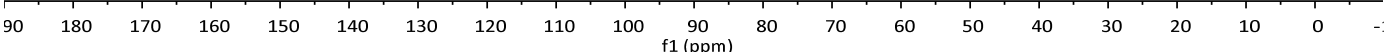


(-)-(3S,4S)-3-Amino-4-ethyl-3-methyl-1-phenyl-3,4-dihydroquinolin-2(1H)-one $((3 S, 4 S)-9)$

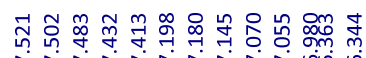

ririsirision

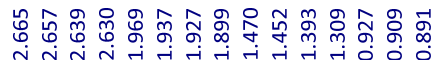

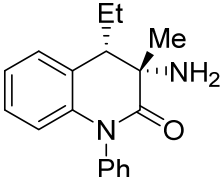

(3S,4S)-9

( ${ }^{1} \mathrm{H} \mathrm{NMR}, 400 \mathrm{MHz}, \mathrm{CDCl}_{3}$ )
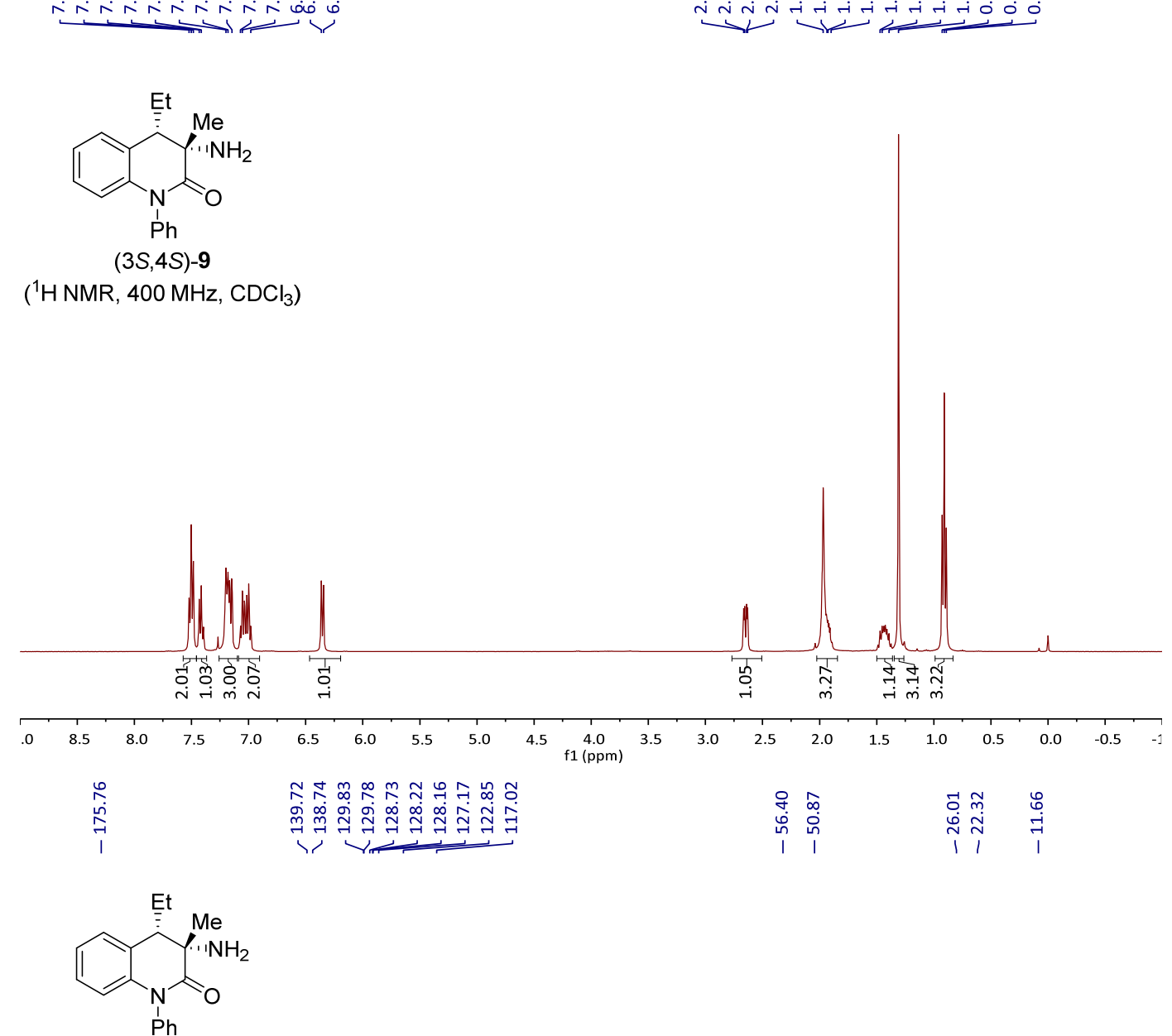

(3S,4S)-9

$\left({ }^{13} \mathrm{C} \mathrm{NMR}, 101 \mathrm{MHz}, \mathrm{CDCl}_{3}\right)$

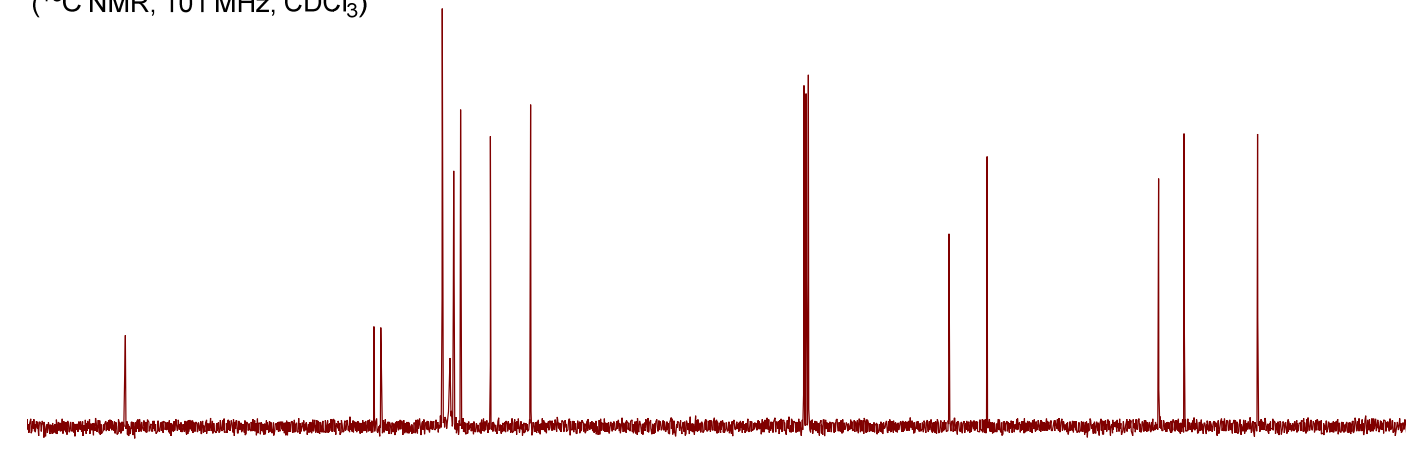

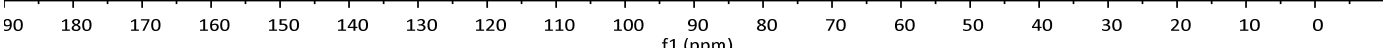


(+)-(3S,4S)-4-ethyl-3-methyl-1-phenyl-1,2,3,4-tetrahydroquinolin-3-amine (MPR3160)

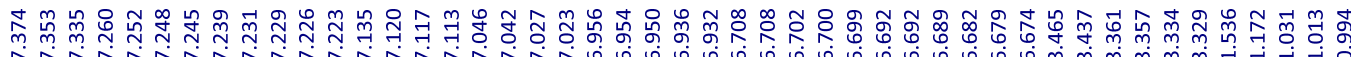

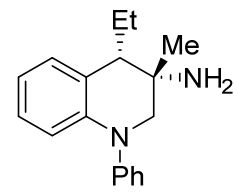

MPR3160

( ${ }^{1} \mathrm{H}$ NMR, $400 \mathrm{MHz}, \mathrm{CDCl}_{3}$ )

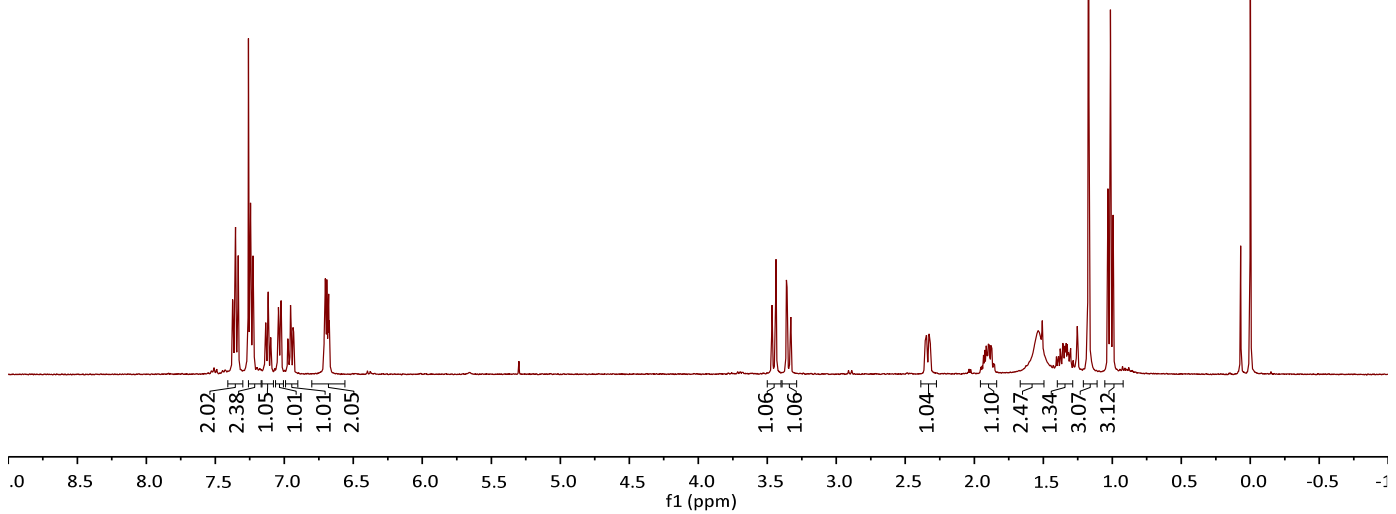


(F) HPLC Charts for Hydrogenation Products

(+)-Ethyl 1,4-dimethyl-2-oxo-1,2,3,4-tetrahydroquinoline-3-carboxylate (3a)
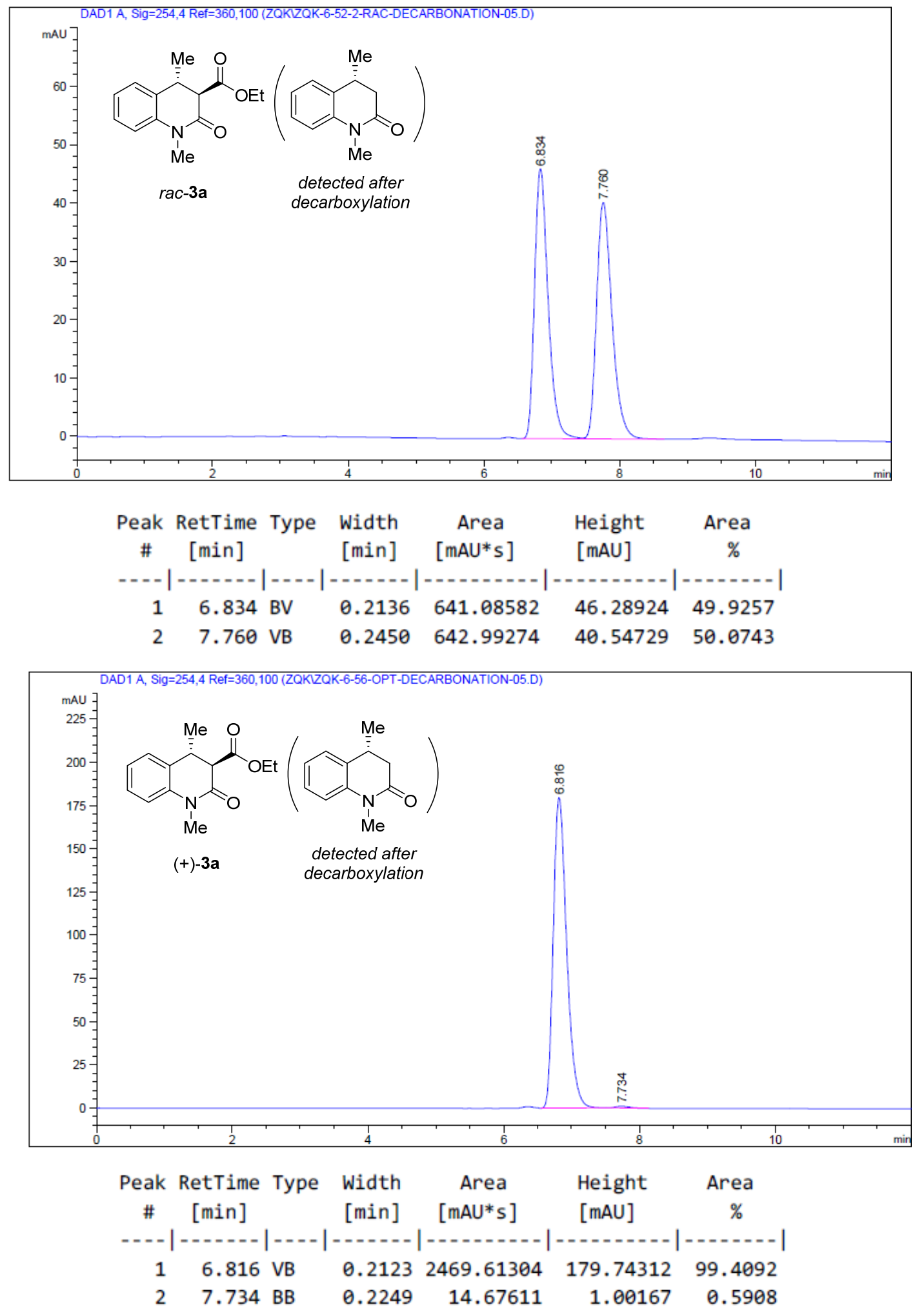
(-)-Ethyl 4-ethyl-1-methyl-2-oxo-1,2,3,4-tetrahydroquinoline-3-carboxylate (3b)
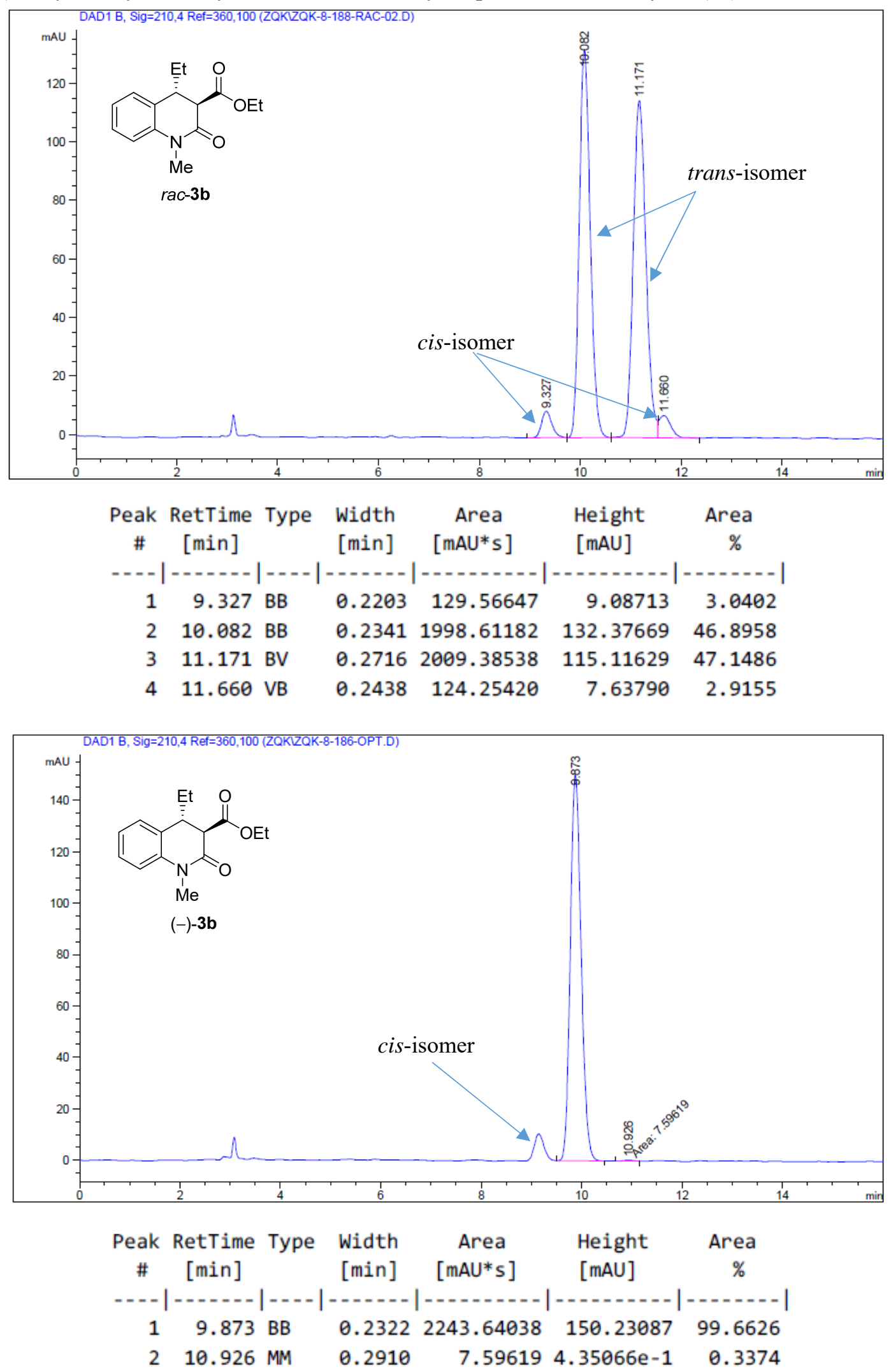
(+)-Ethyl 1-methyl-2-oxo-4-propyl-1,2,3,4-tetrahydroquinoline-3-carboxylate (3c)

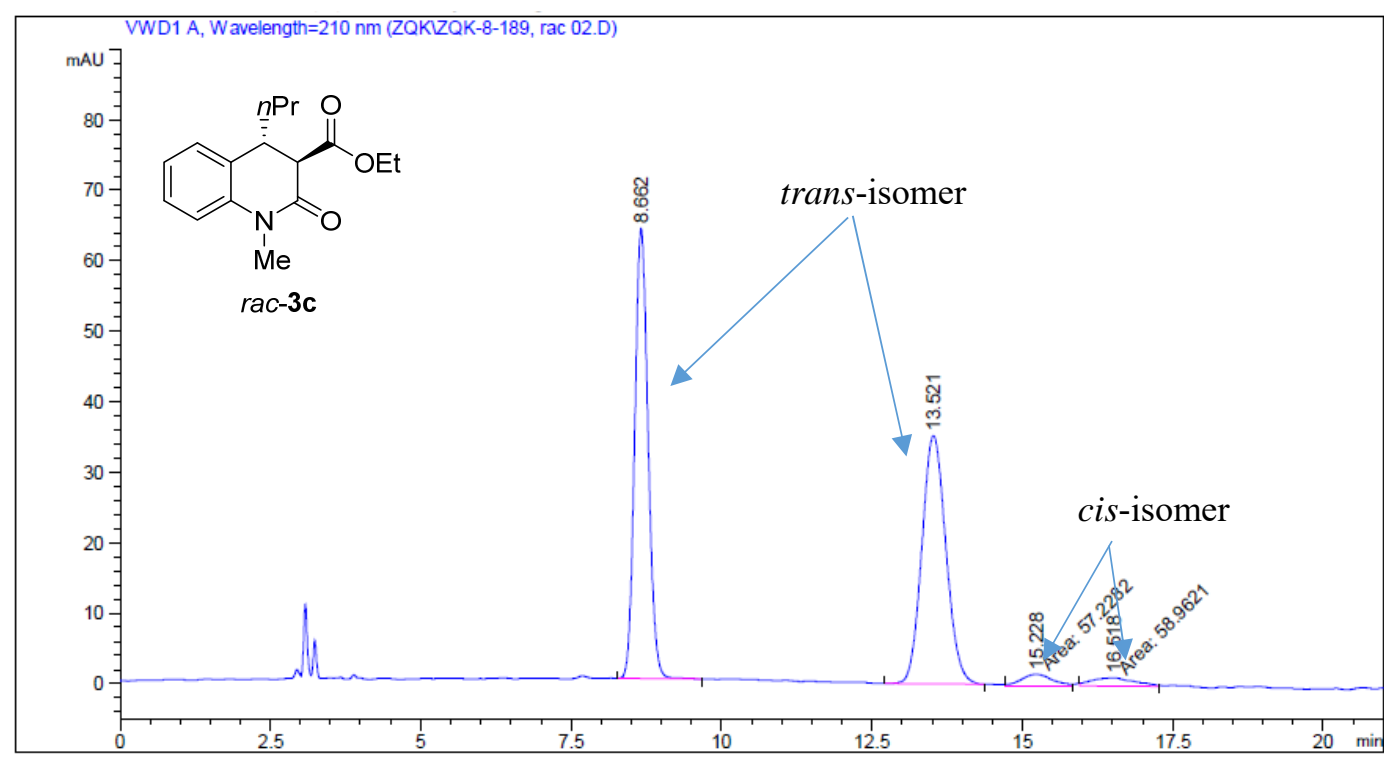

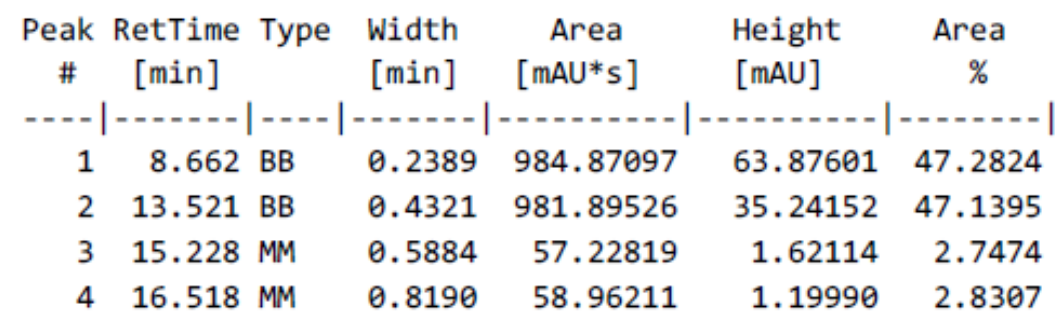

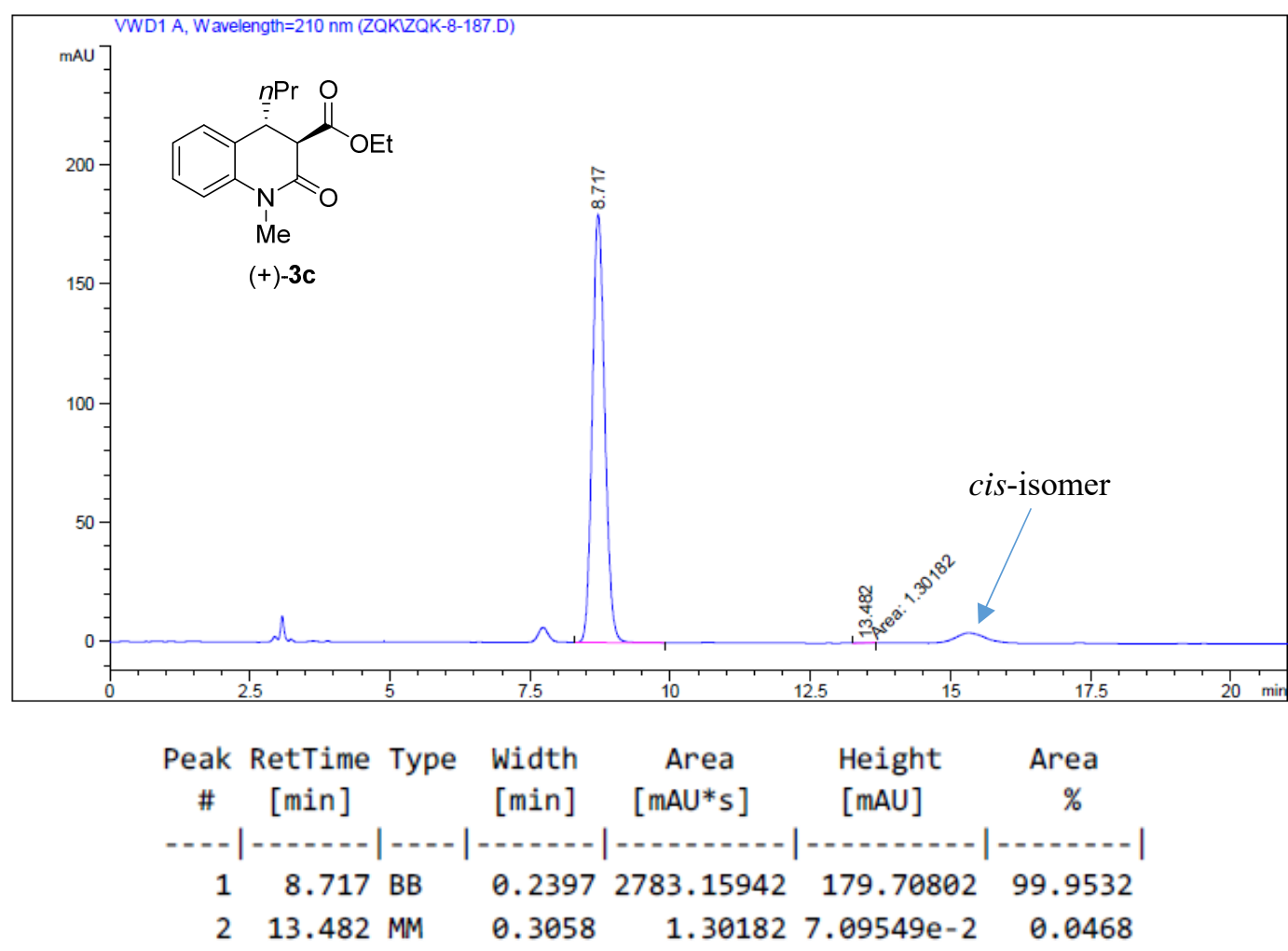


(+)-Ethyl 4-isobutyl-1-methyl-2-oxo-1,2,3,4-tetrahydroquinoline-3-carboxylate (3d)
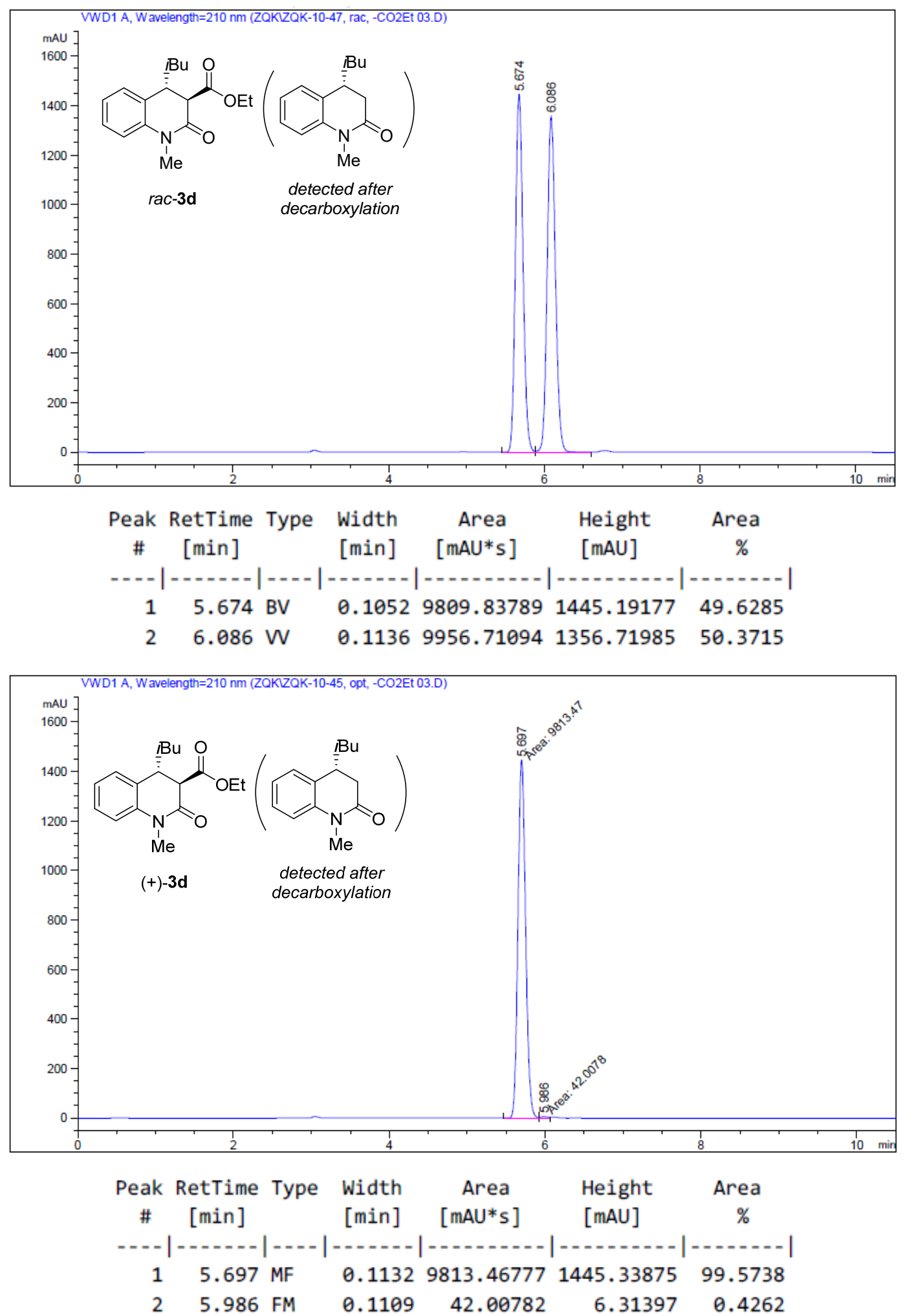
(+)-Ethyl 4-benzyl-1-methyl-2-oxo-1,2,3,4-tetrahydroquinoline-3-carboxylate (3e)
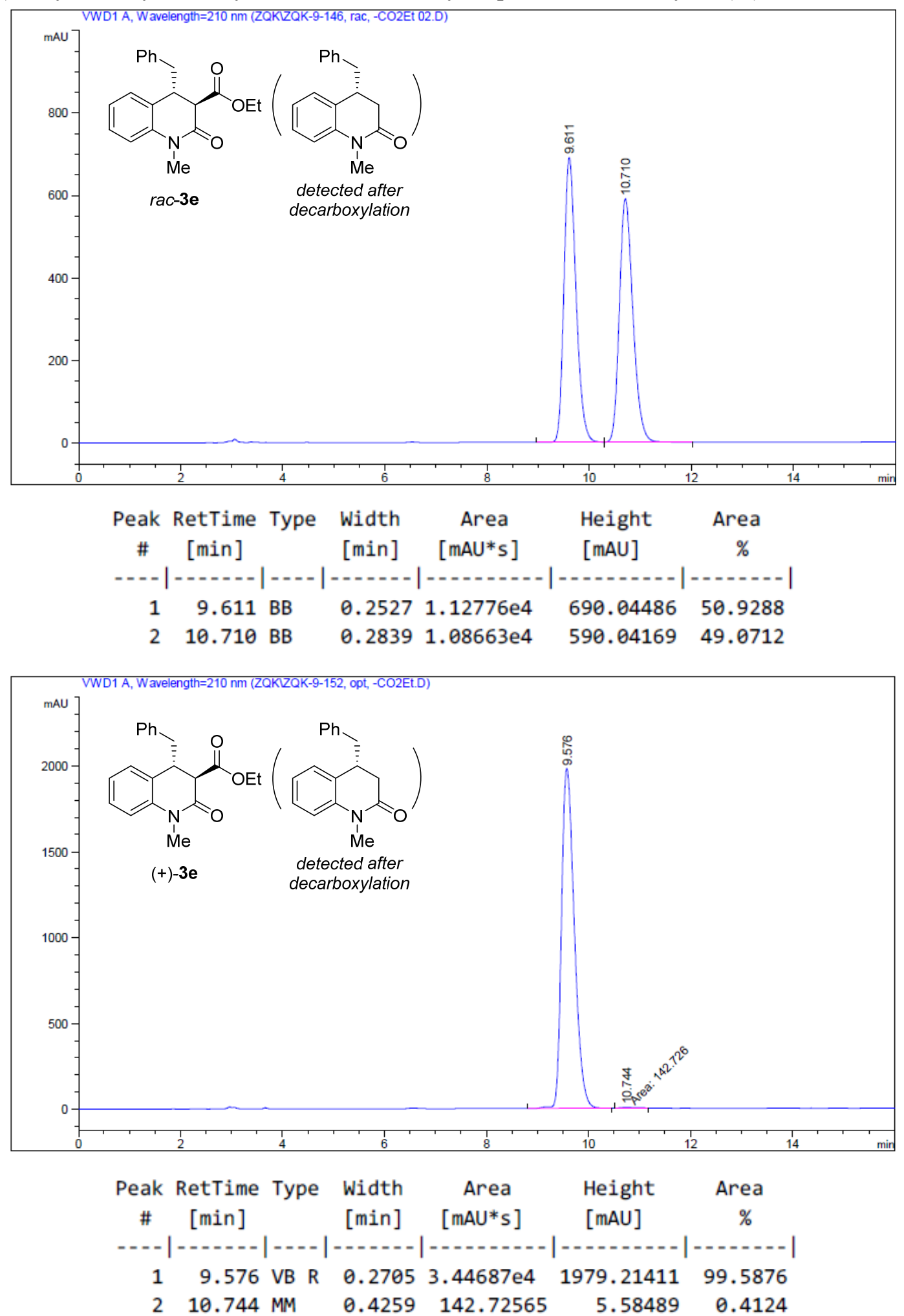
(+)-Ethyl 1-methyl-2-oxo-4-phenethyl-1,2,3,4-tetrahydroquinoline-3-carboxylate (3f)
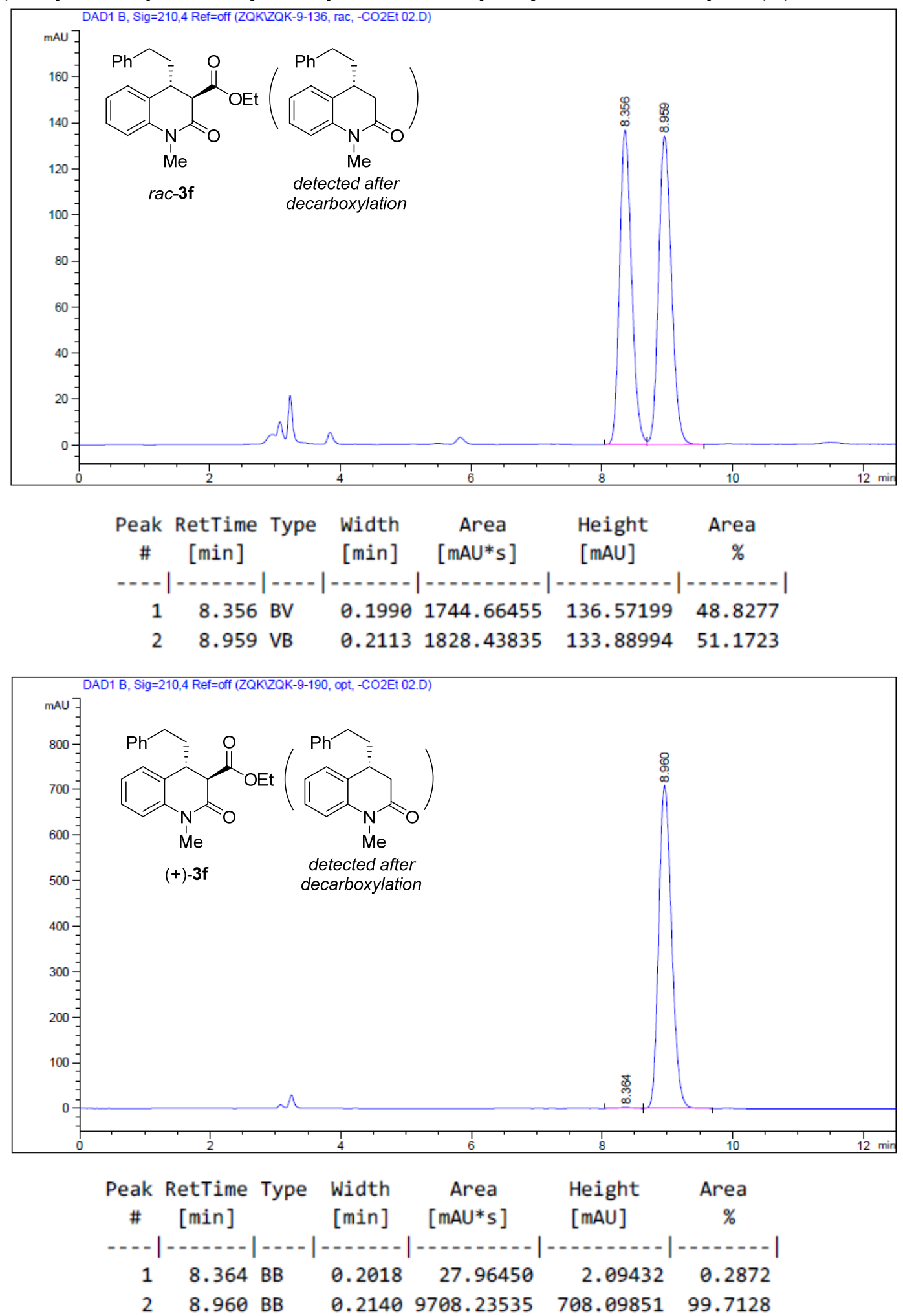
(+)-Ethyl 1-methyl-2-oxo-4-pentyl-1,2,3,4-tetrahydroquinoline-3-carboxylate (3g)
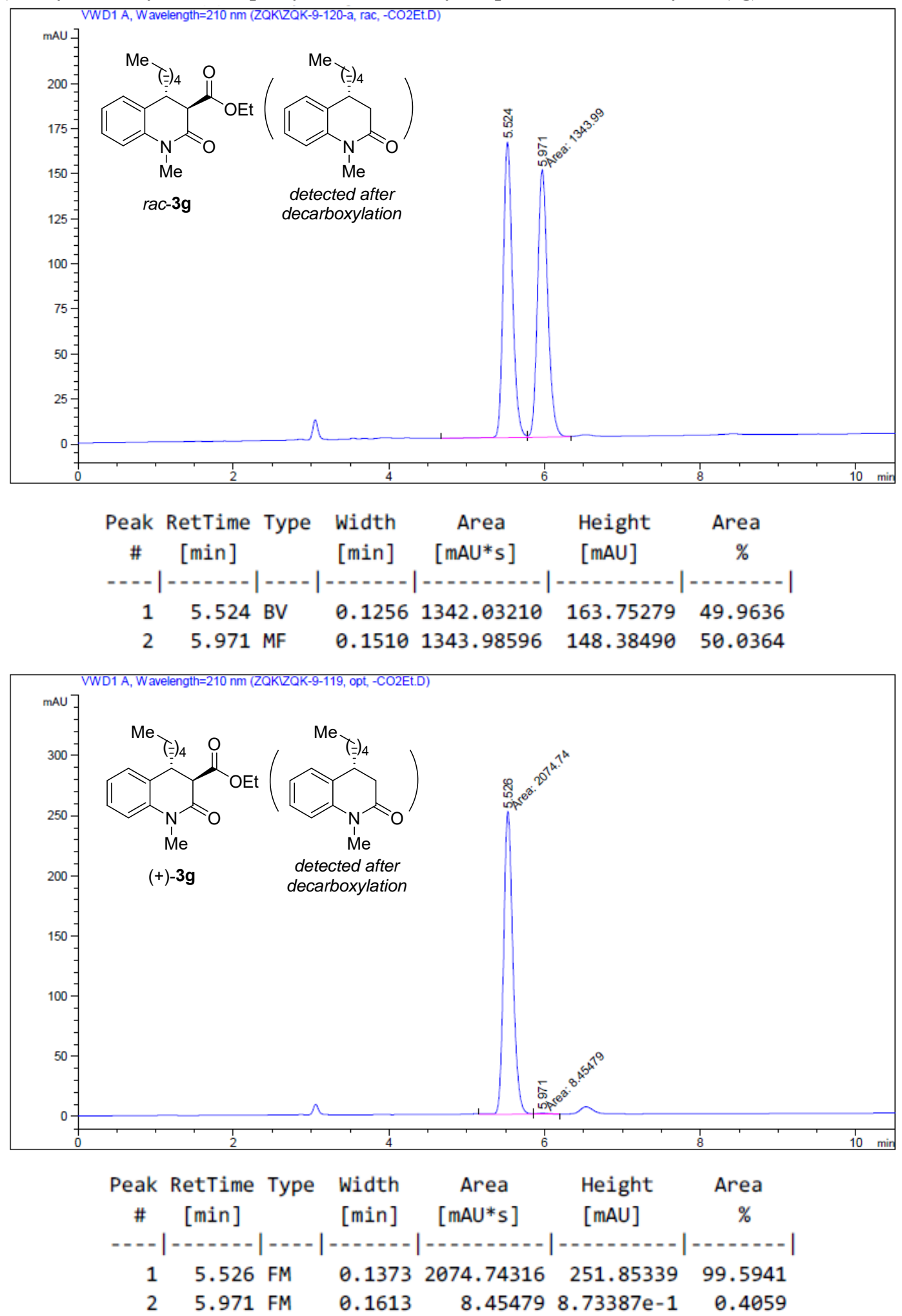
(+)-Ethyl 4-cyclopropyl-1-methyl-2-oxo-1,2,3,4-tetrahydroquinoline-3-carboxylate (3h)
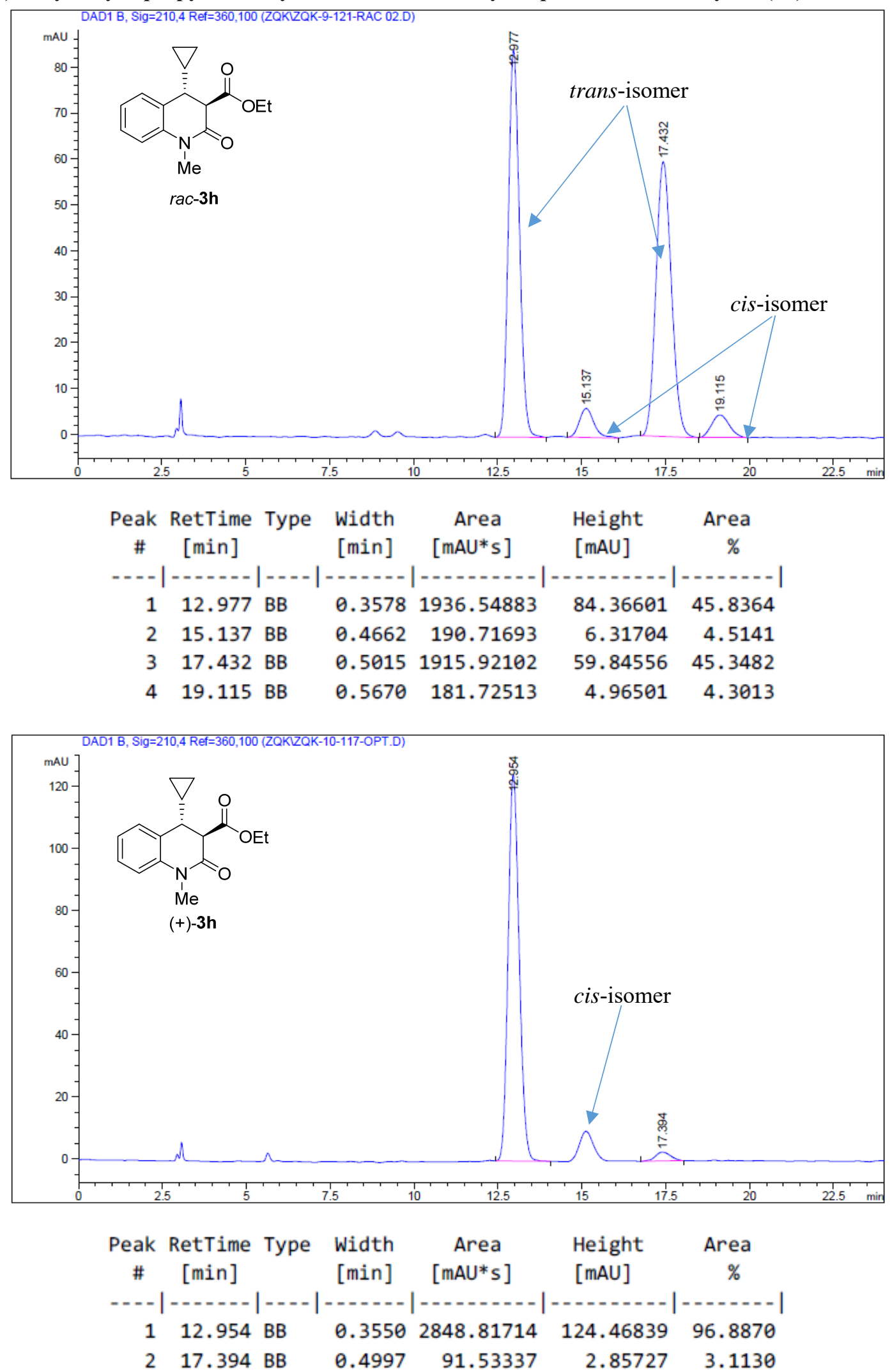
(-)-(3S,4R)-Ethyl 1-methyl-2-oxo-4-phenyl-1,2,3,4-tetrahydroquinoline-3-carboxylate ((3S,4R)-3i)
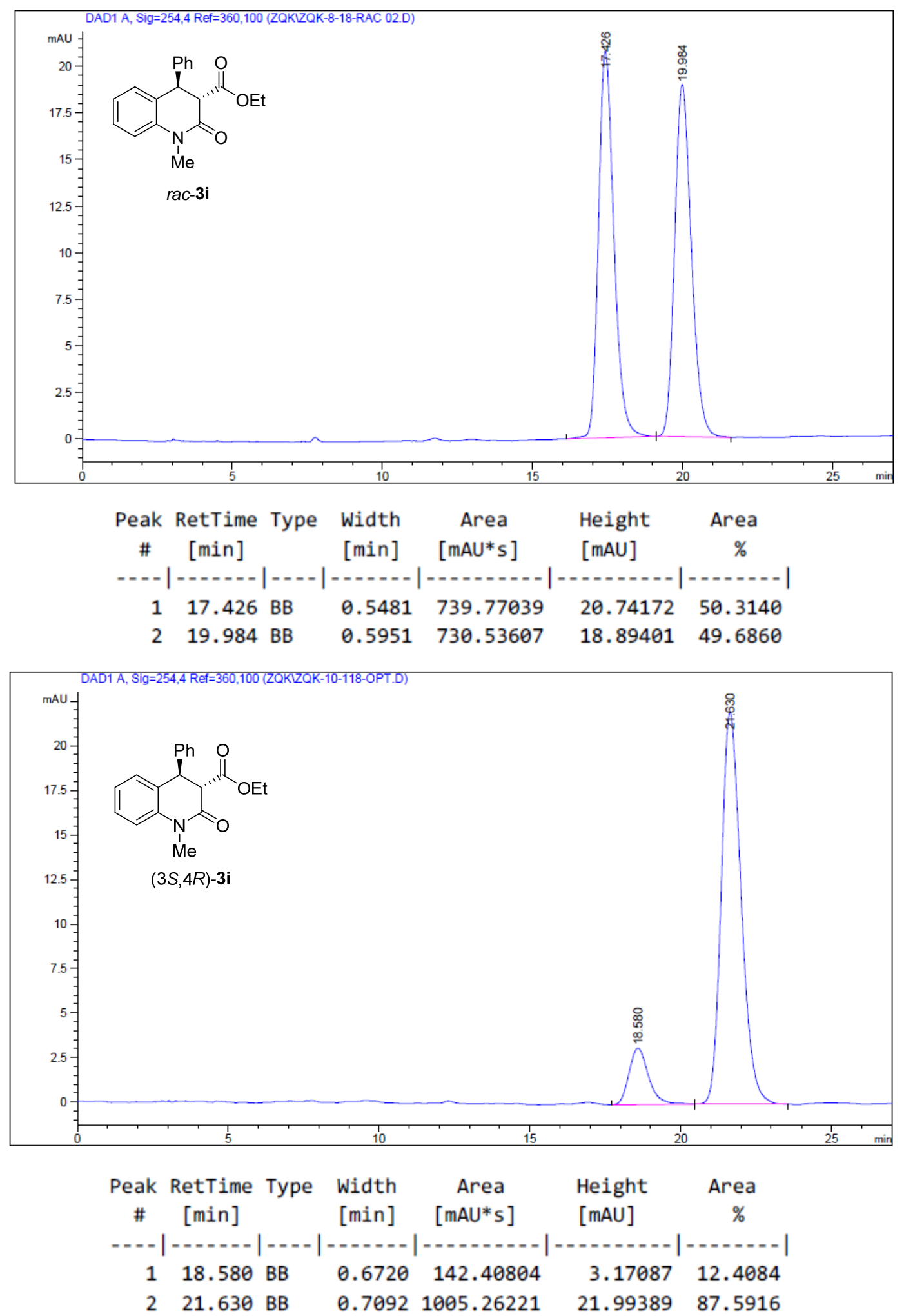
(+)-Ethyl 6-bromo-1,4-dimethyl-2-oxo-1,2,3,4-tetrahydroquinoline-3-carboxylate (3j)
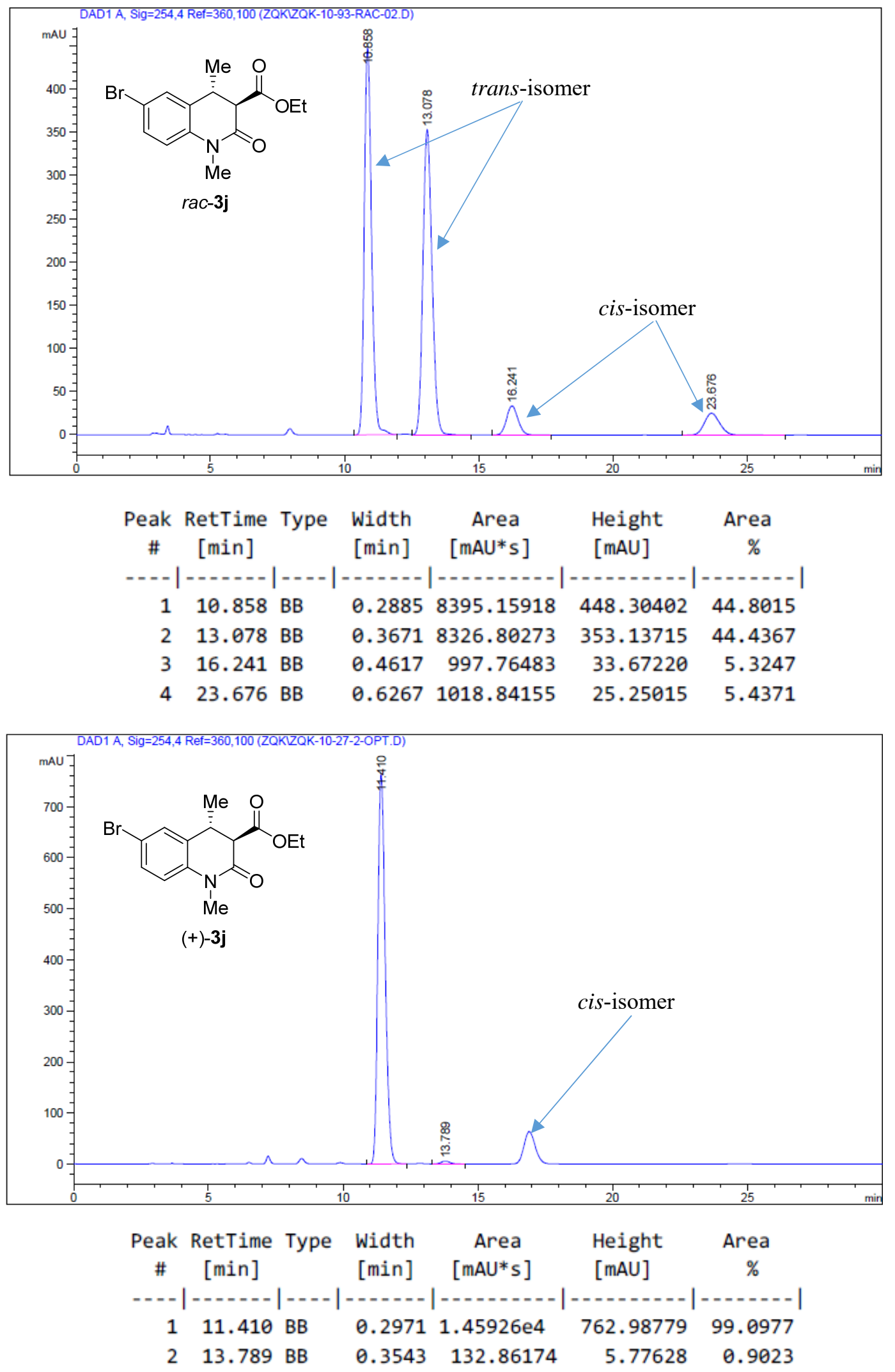
(-)-Ethyl 6-methoxy-1,4-dimethyl-2-oxo-1,2,3,4-tetrahydroquinoline-3-carboxylate (3k)
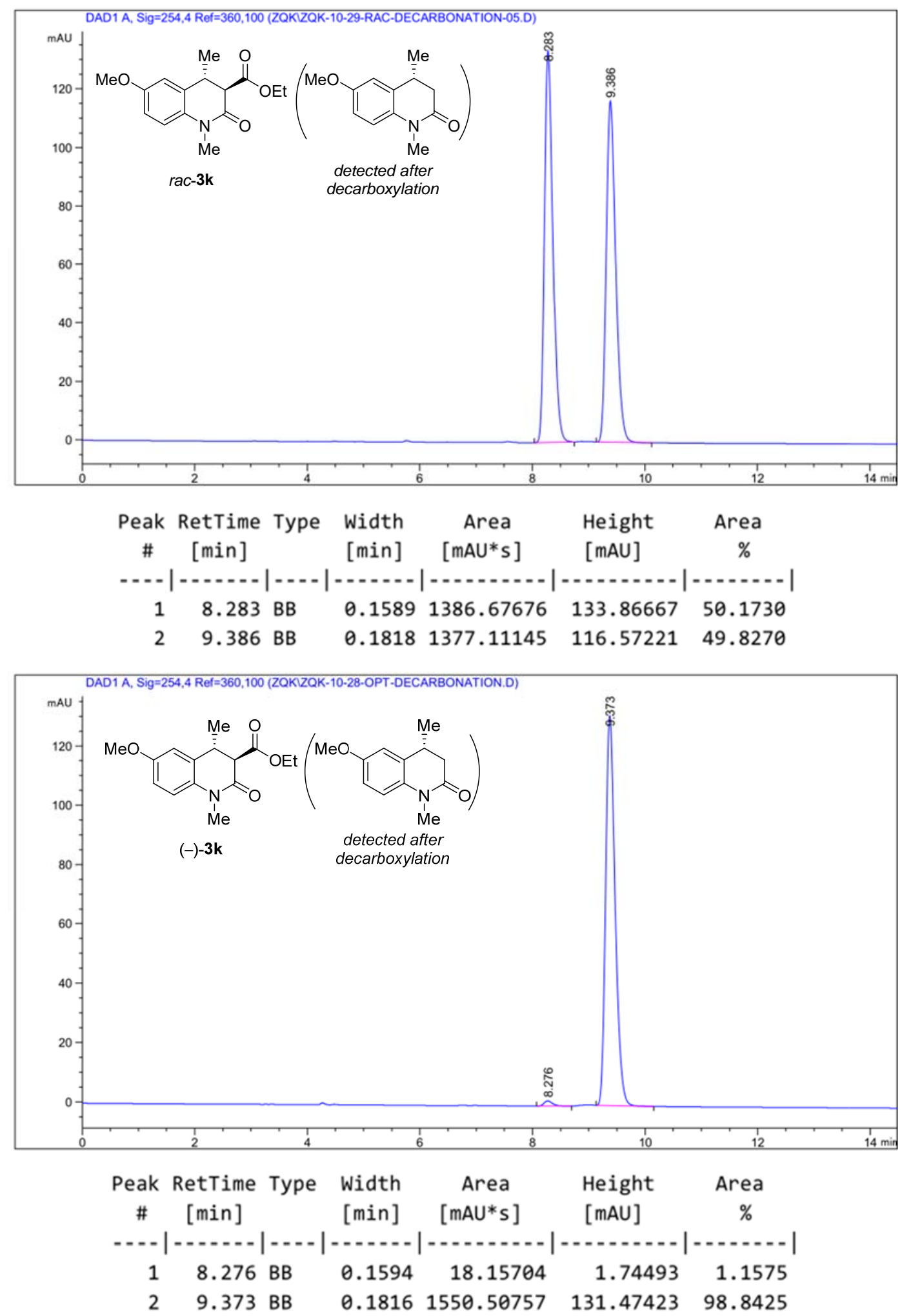
(+)-Ethyl 1-ethyl-4-methyl-2-oxo-1,2,3,4-tetrahydroquinoline-3-carboxylate (3I)
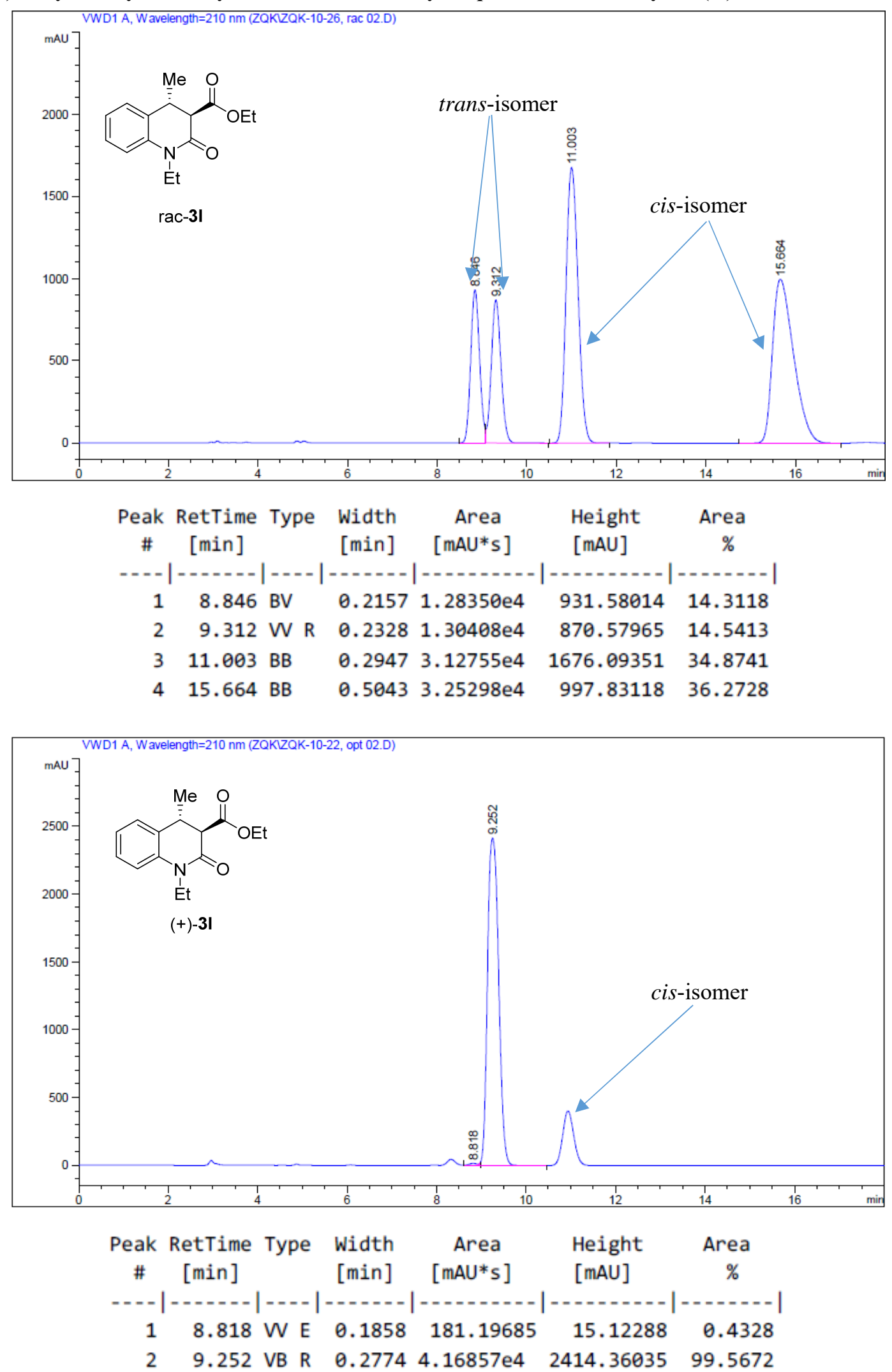
(+)-Ethyl 1-benzyl-4-methyl-2-oxo-1,2,3,4-tetrahydroquinoline-3-carboxylate (3m)
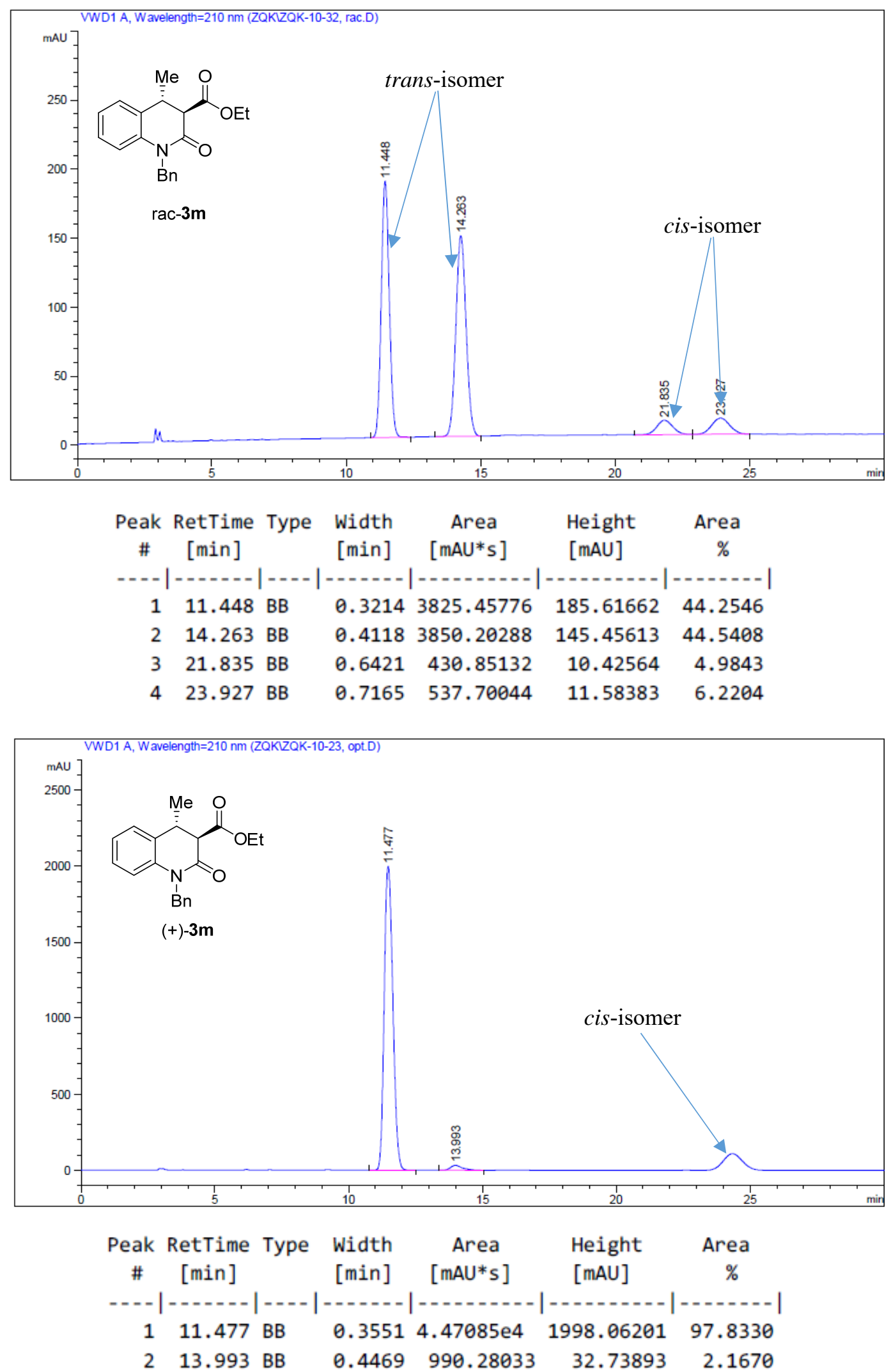
(-)-Ethyl 4-methyl-2-oxo-1-phenyl-1,2,3,4-tetrahydroquinoline-3-carboxylate (3n)
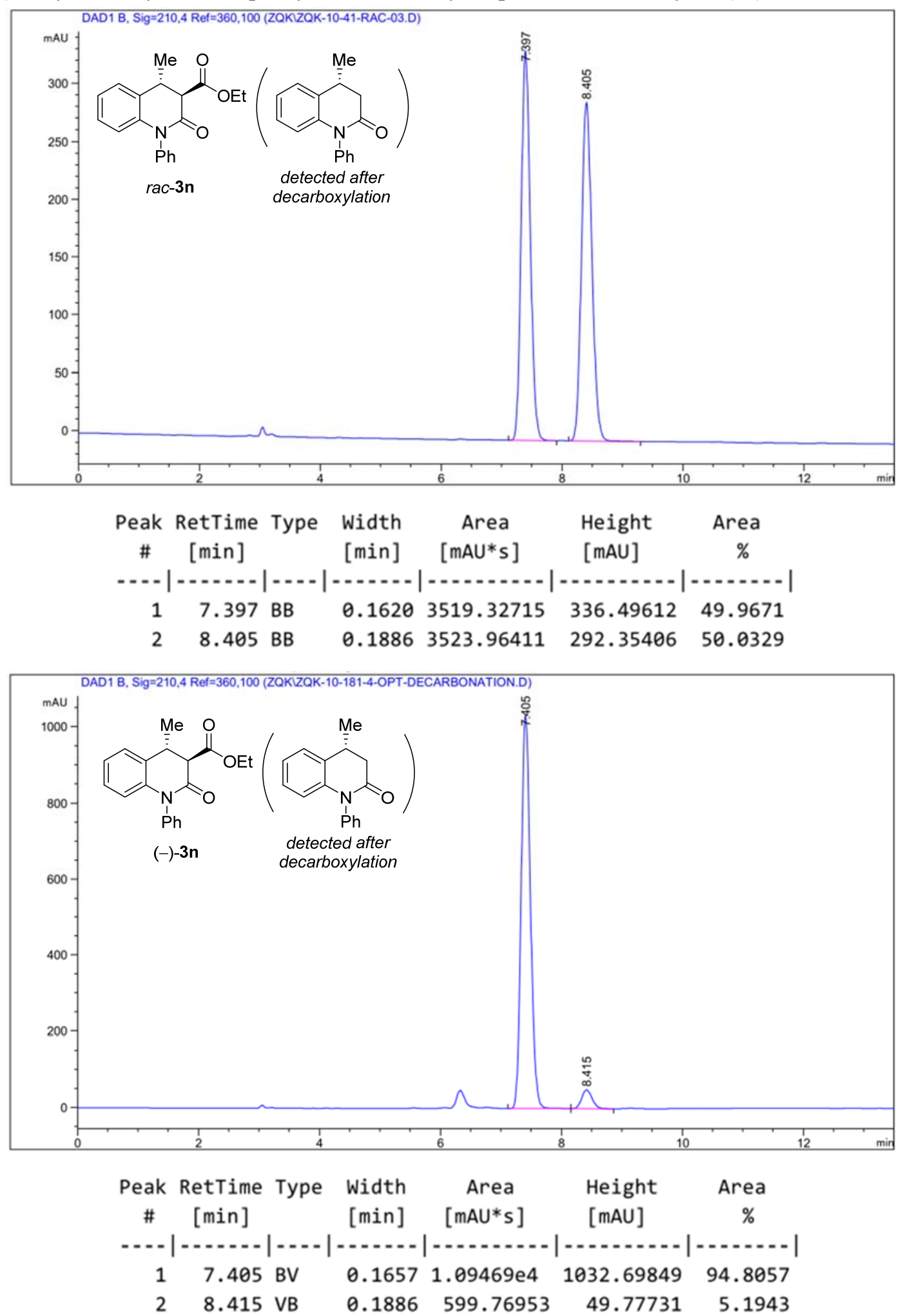
(-)-(3R,4R)-ethyl 4-ethyl-2-oxo-1-phenyl-1,2,3,4-tetrahydroquinoline-3-carboxylate $((3 R, 4 R)-30)$

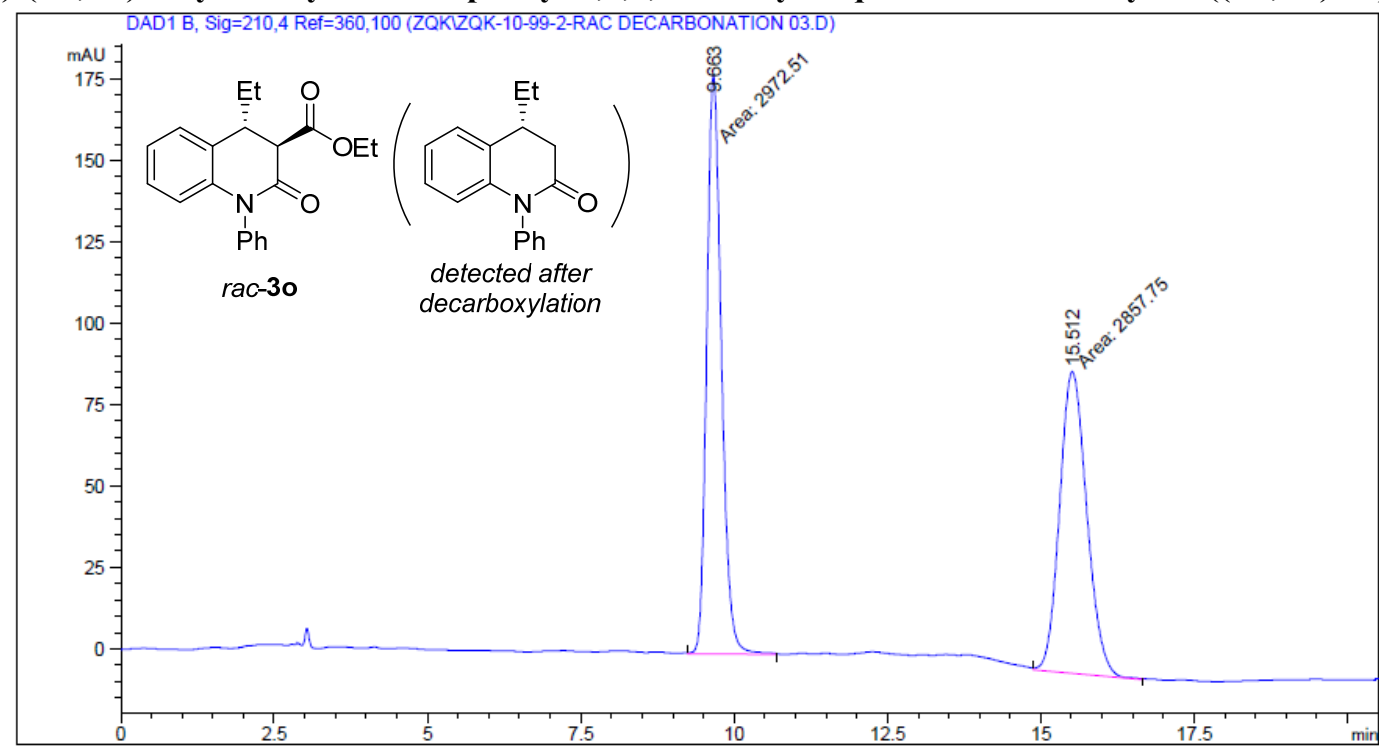

\begin{tabular}{cccccc}
$\begin{array}{c}\text { Peak RetTime Type } \\
\text { \# }\end{array}$ & $\begin{array}{c}\text { Width } \\
\text { [min] }\end{array}$ & $\begin{array}{c}\text { Area } \\
\text { [min] }\end{array}$ & $\begin{array}{c}\text { Height } \\
\text { [mAU] }\end{array}$ & $\begin{array}{c}\text { Area } \\
\%\end{array}$ \\
\hline 1 & 9.663 MM & 0.2787 & 2972.51074 & 177.74701 & 50.9841 \\
2 & 15.512 MM & 0.5140 & 2857.75415 & 92.66589 & 49.0159
\end{tabular}

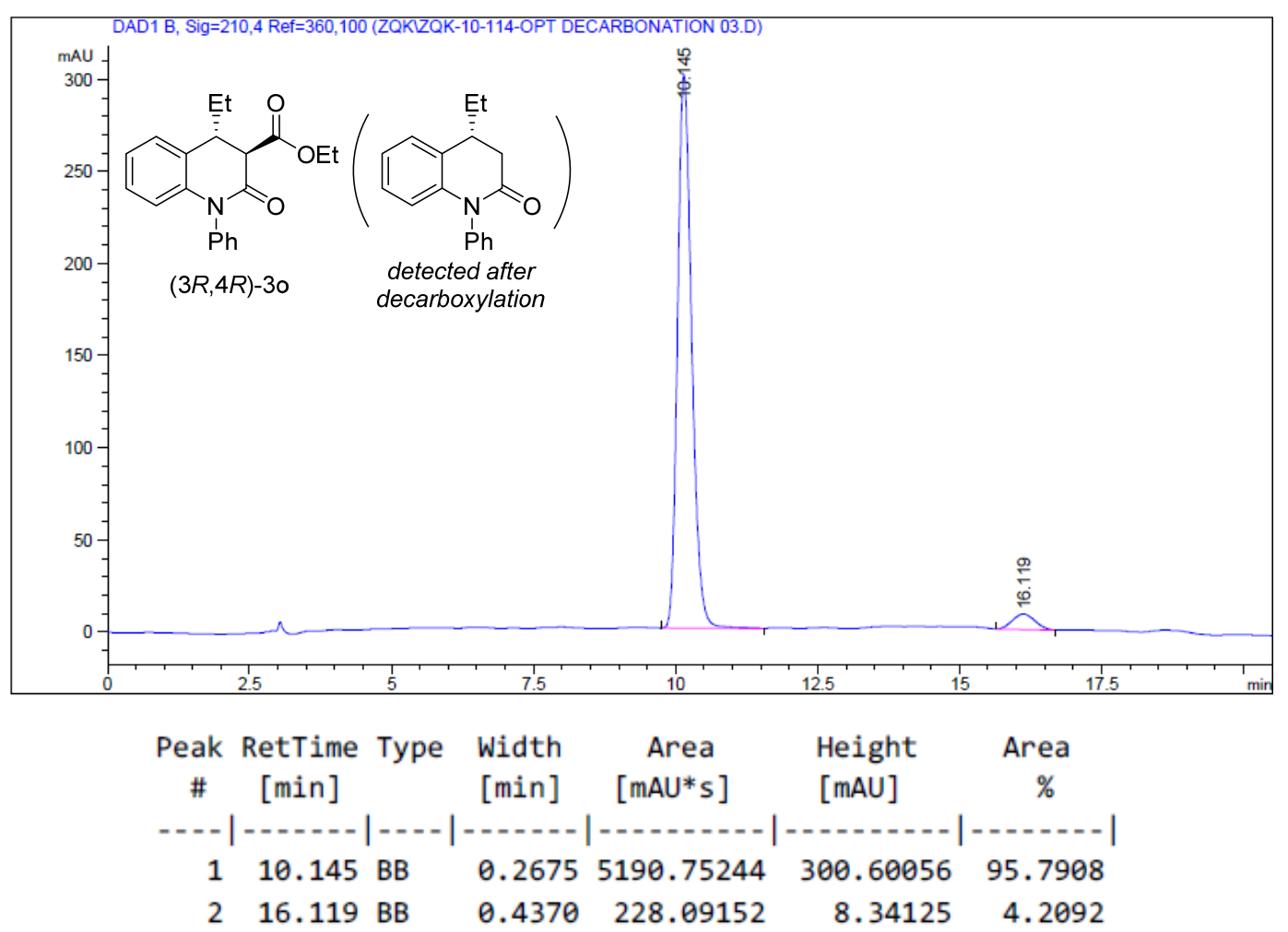


(+)-Ethyl 4-methyl-2-oxo-1,2,3,4-tetrahydroquinoline-3-carboxylate (3p)
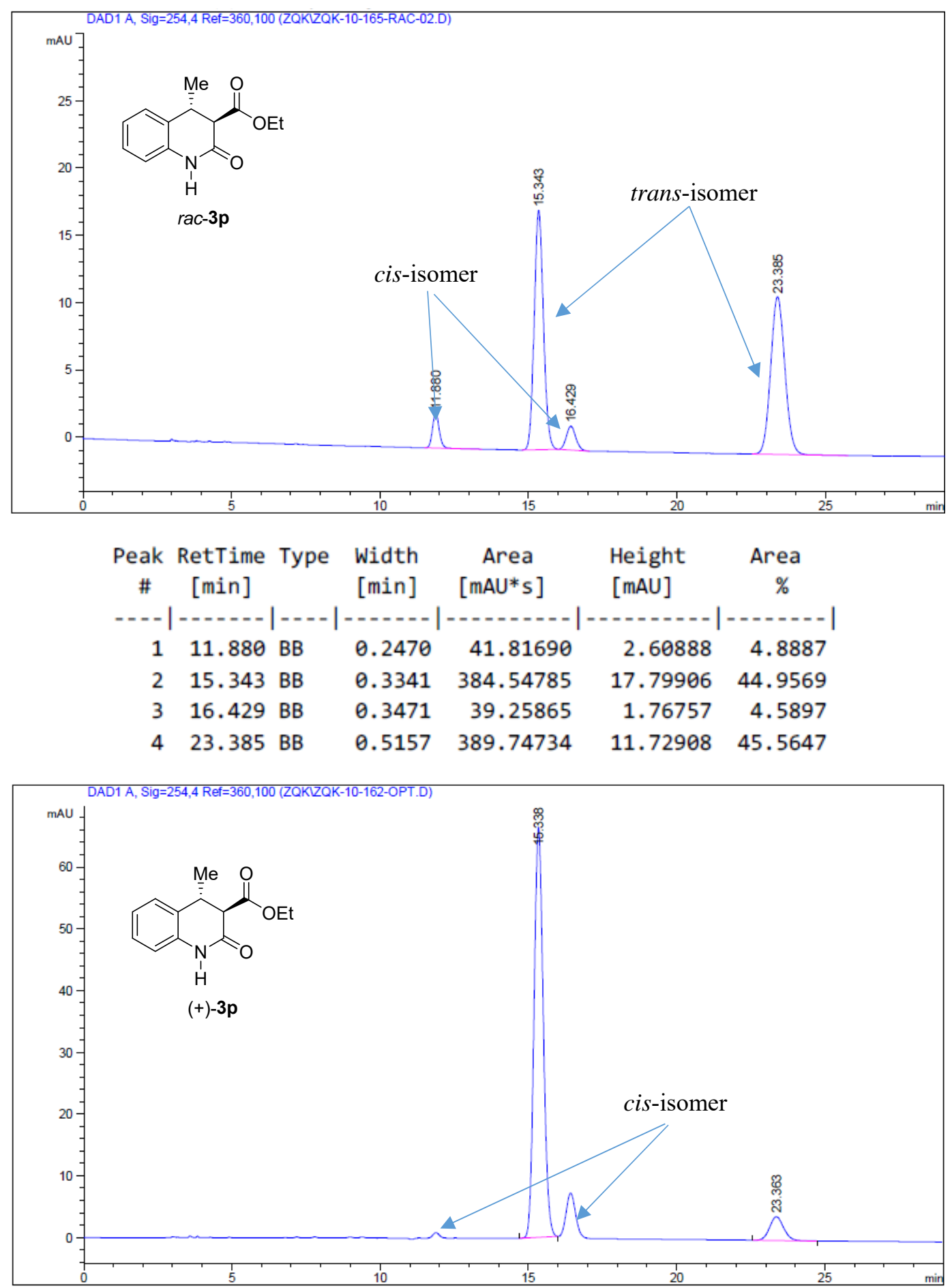

\begin{tabular}{cccccc}
$\begin{array}{c}\text { Peak RetTime Type } \\
\text { \# } \\
{[\text { Win] }}\end{array}$ & $\begin{array}{c}\text { Width } \\
{[\text { min] }}\end{array}$ & $\begin{array}{c}\text { Area } \\
{[\text { mAU* }]}\end{array}$ & $\begin{array}{l}\text { Height } \\
{[\mathrm{mAU}]}\end{array}$ & $\begin{array}{c}\text { Area } \\
\%\end{array}$ \\
\hdashline & 15.338 BB & 0.3317 & 1420.36426 & 66.37112 & 91.8289 \\
2 & 23.363 BB & 0.5067 & 126.38706 & 3.83287 & 8.1711
\end{tabular}


(+)-(3S,4R)-Ethyl-4-methyl-2-oxochromane-3-carboxylate (5a)
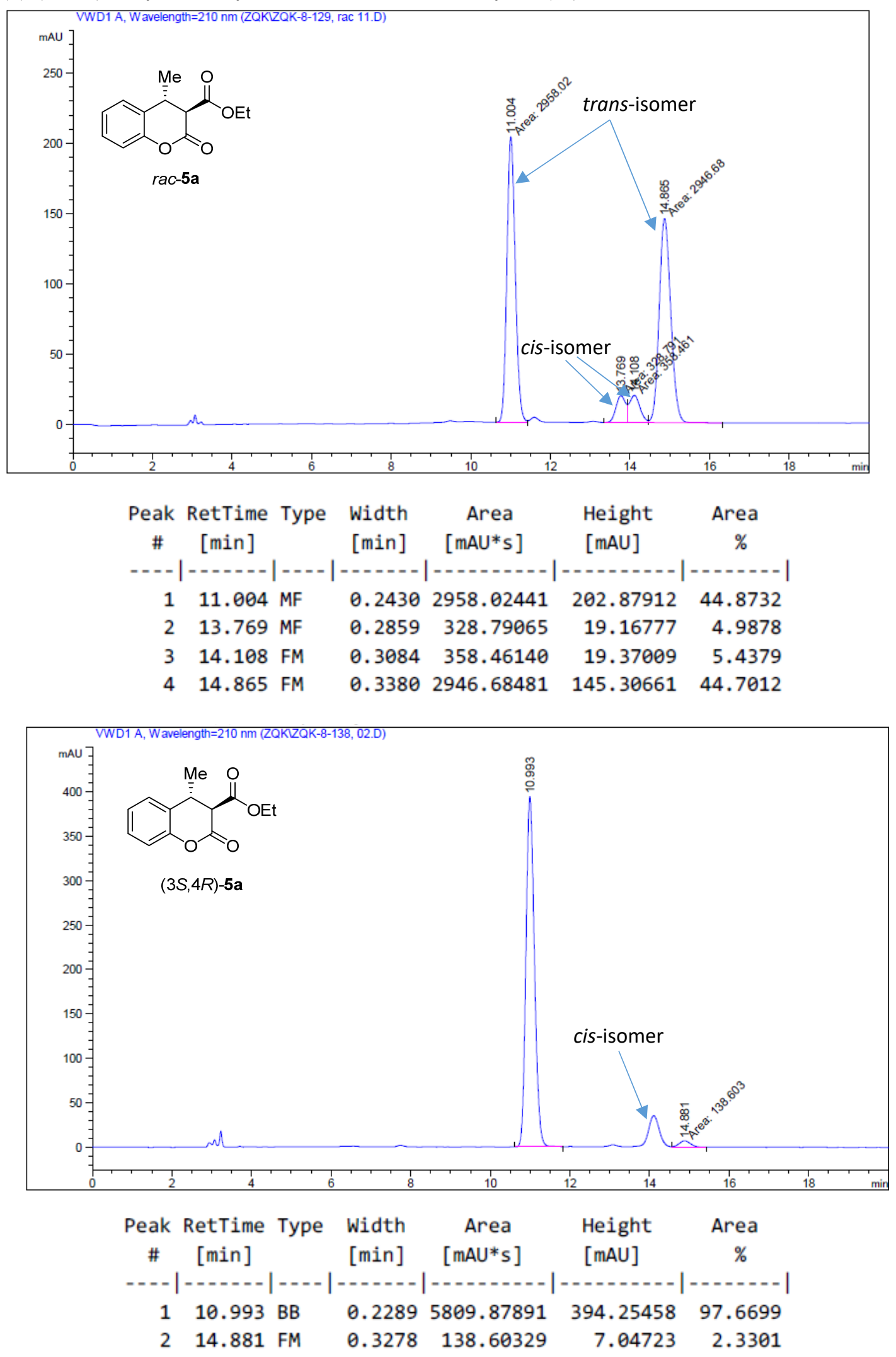
(+)-(3S,4R)-Ethyl-4-ethyl-2-oxochromane-3-carboxylate (5b)
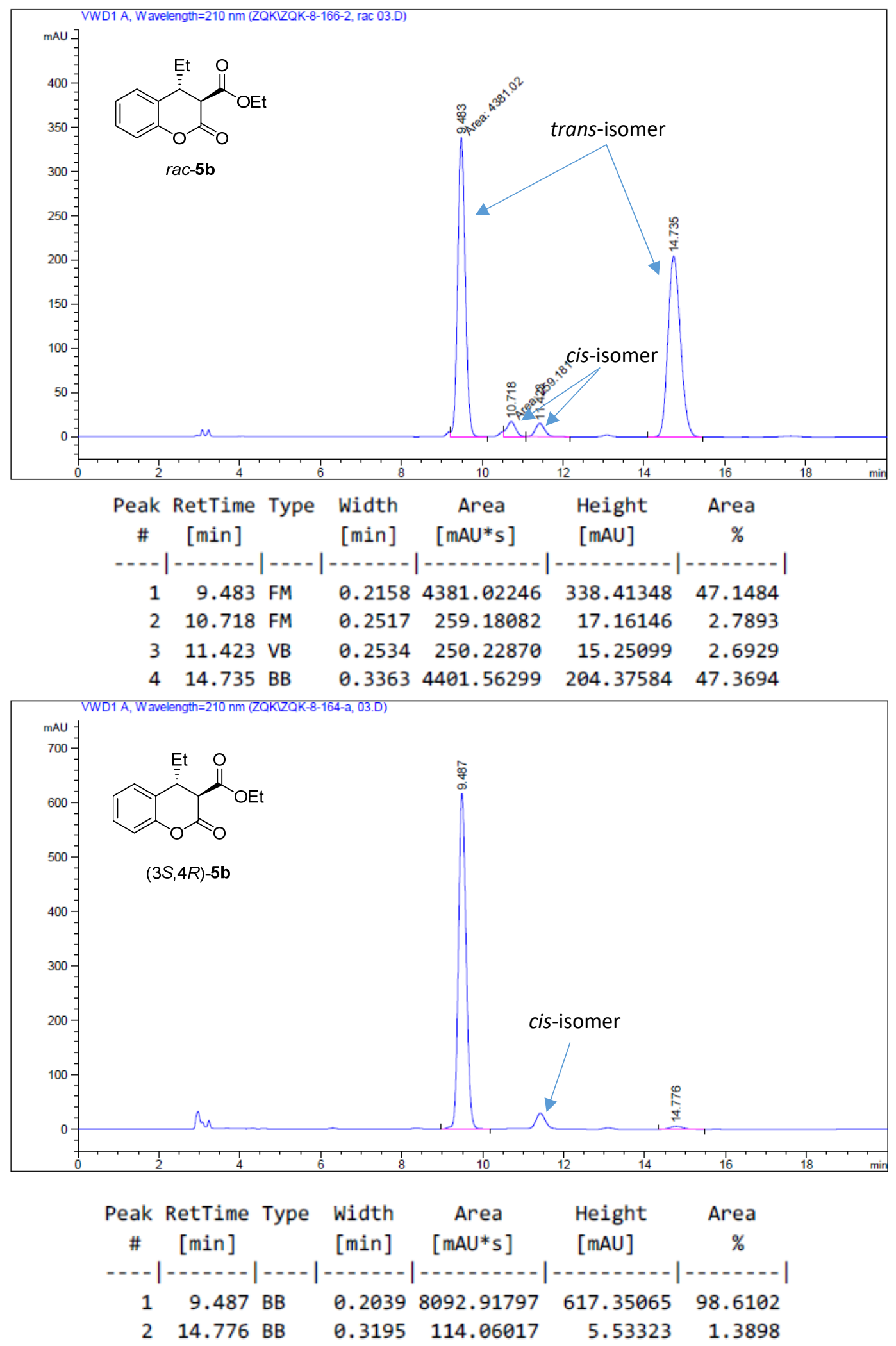
(+)-Ethyl 2-oxo-4-propylchromane-3-carboxylate (5c)
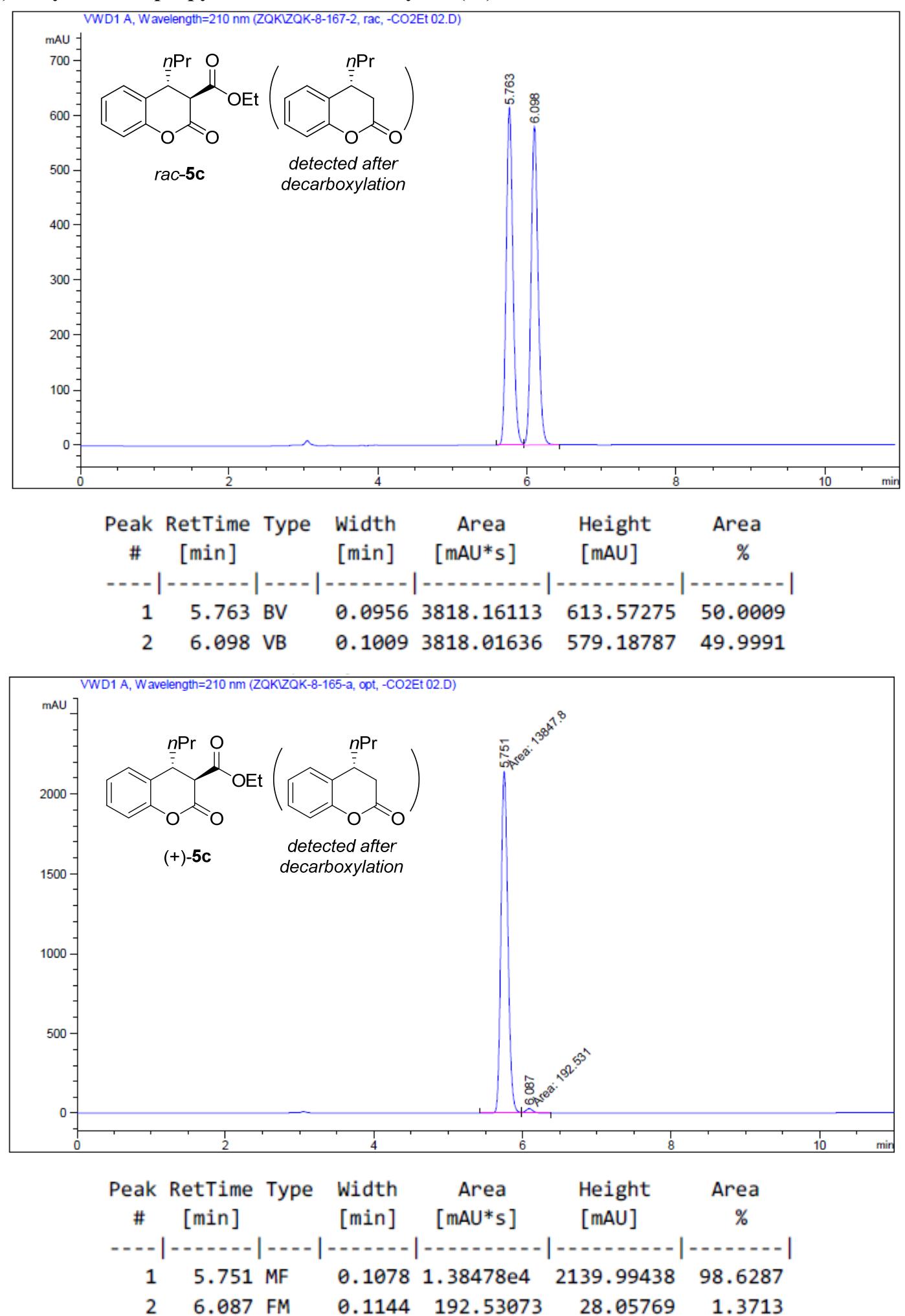
(+)-Ethyl-4-butyl-2-oxochromane-3-carboxylate (5d)
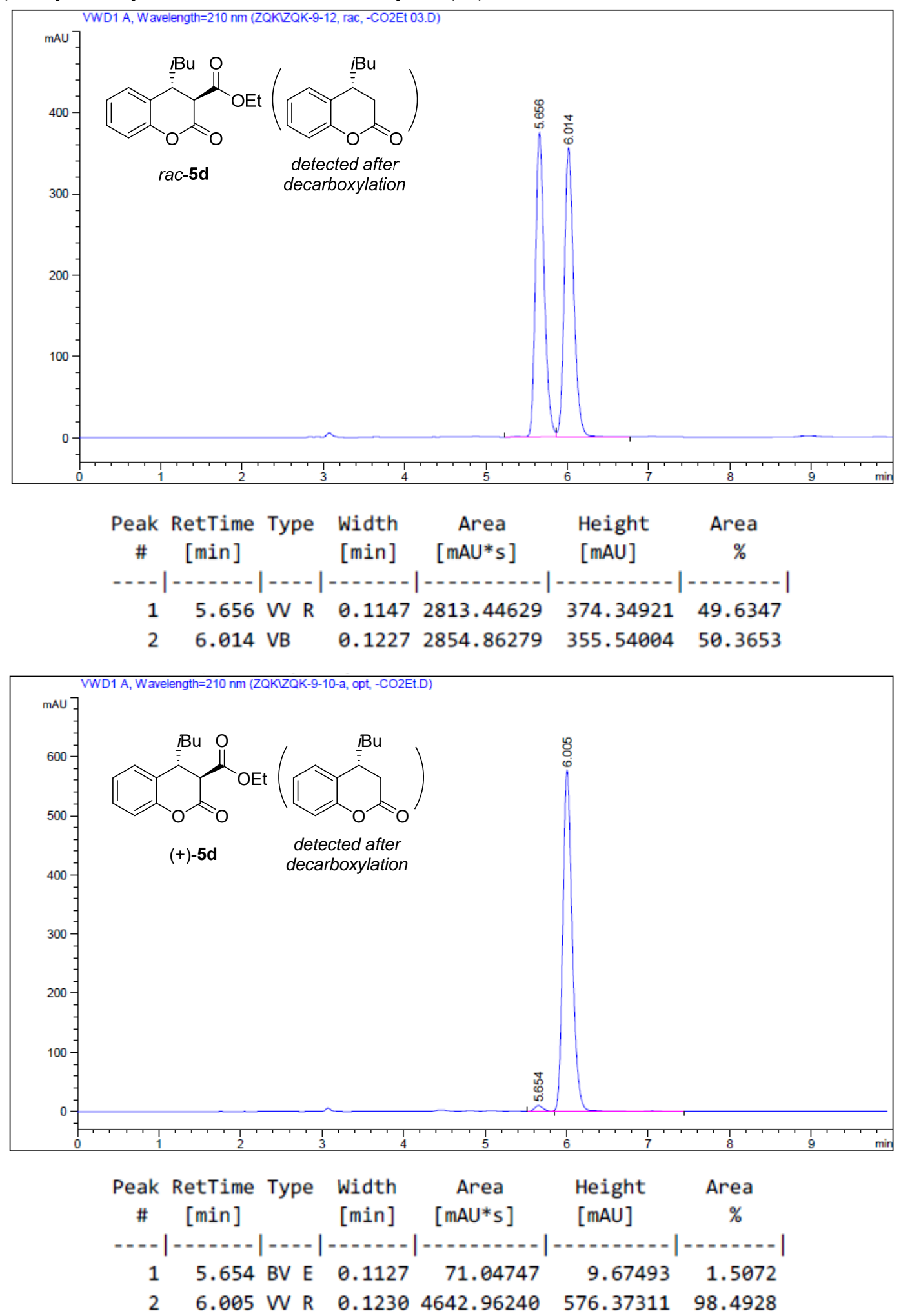
(-)-Ethyl-4-isopropyl-2-oxochromane-3-carboxylate (5e)
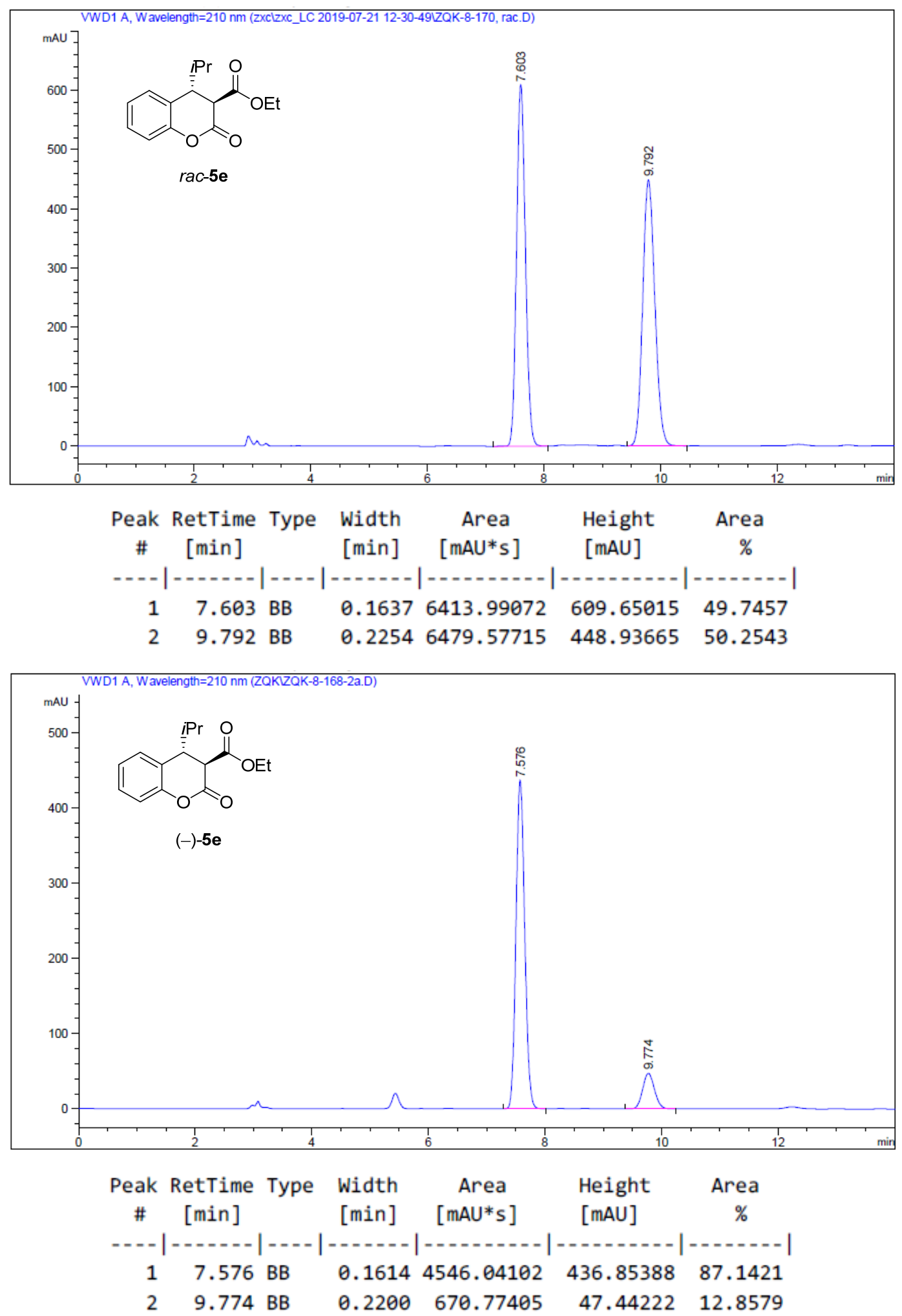
(-)-Ethyl-4-cyclohexyl-2-oxochromane-3-carboxylate (5f)
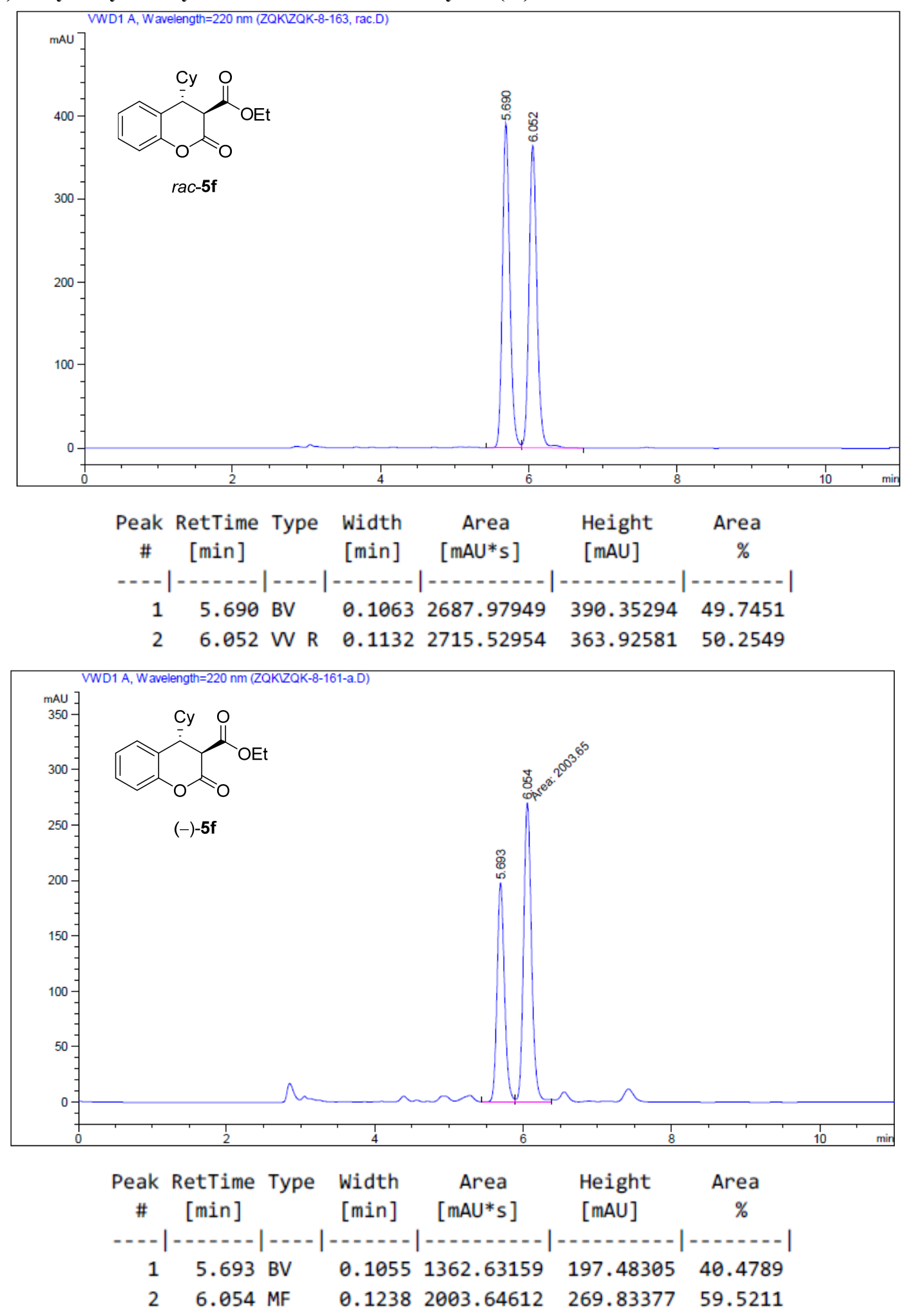
(+)-Ethyl-4-benzyl-2-oxochromane-3-carboxylate (5g)

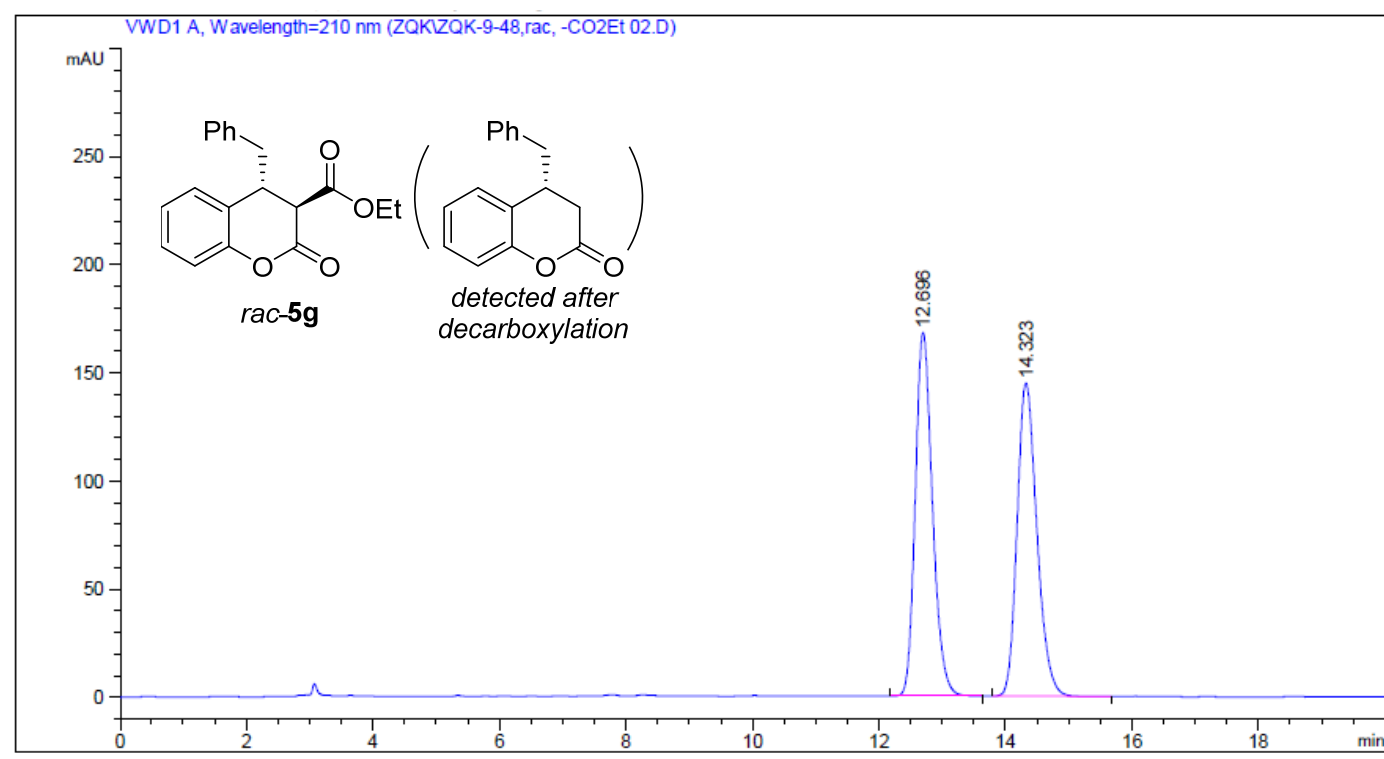
Peak RetTime Type Width Area Height Area
\# [min]
[min] [mAU*s] [mAU]
$\%$

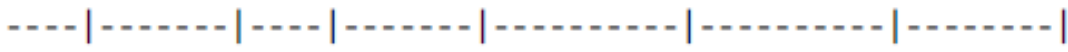
112.696 BB
$\begin{array}{lllll}0.2893 & 3173.82495 & 168.09372 & 50.2157\end{array}$
$214.323 \mathrm{BB}$
$\begin{array}{llll}0.3338 & 3146.55688 & 144.65884 & 49.7843\end{array}$

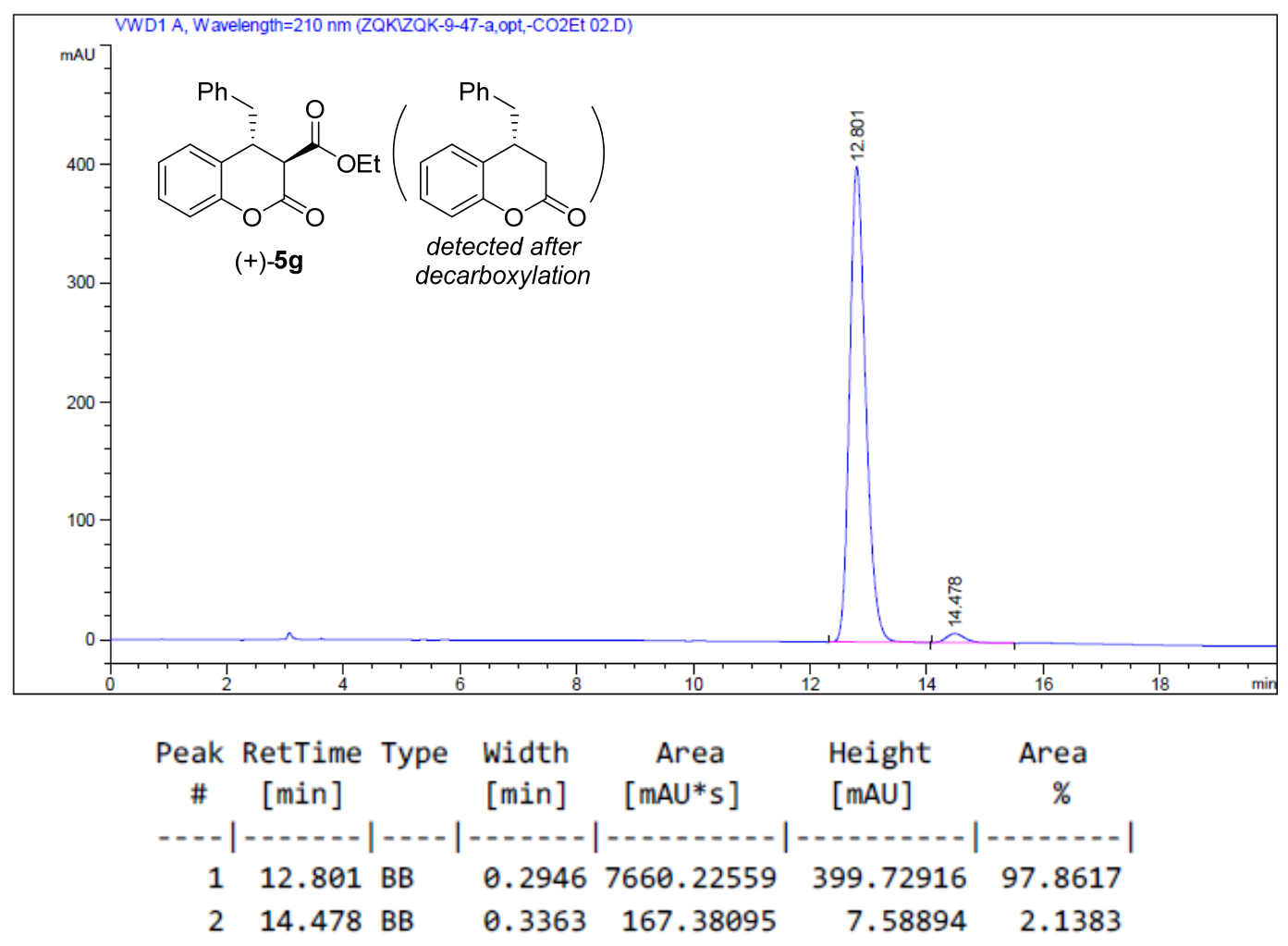


(+)-Ethyl-2-oxo-4-phenethylchromane-3-carboxylate (5h)
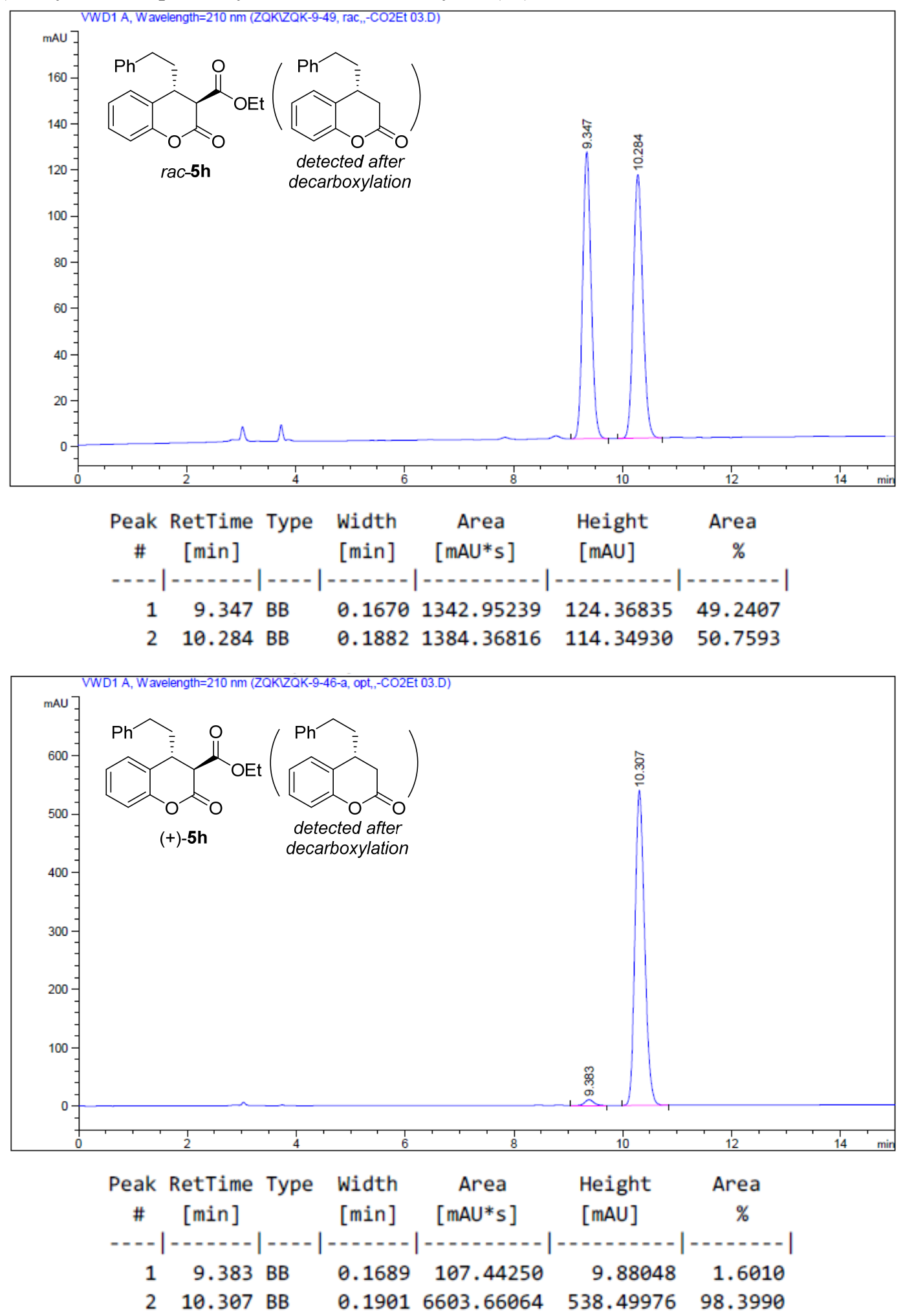
(+)-Ethyl-4-(4-ethoxy-4-oxobutyl)-2-oxochromane-3-carboxylate (5i)
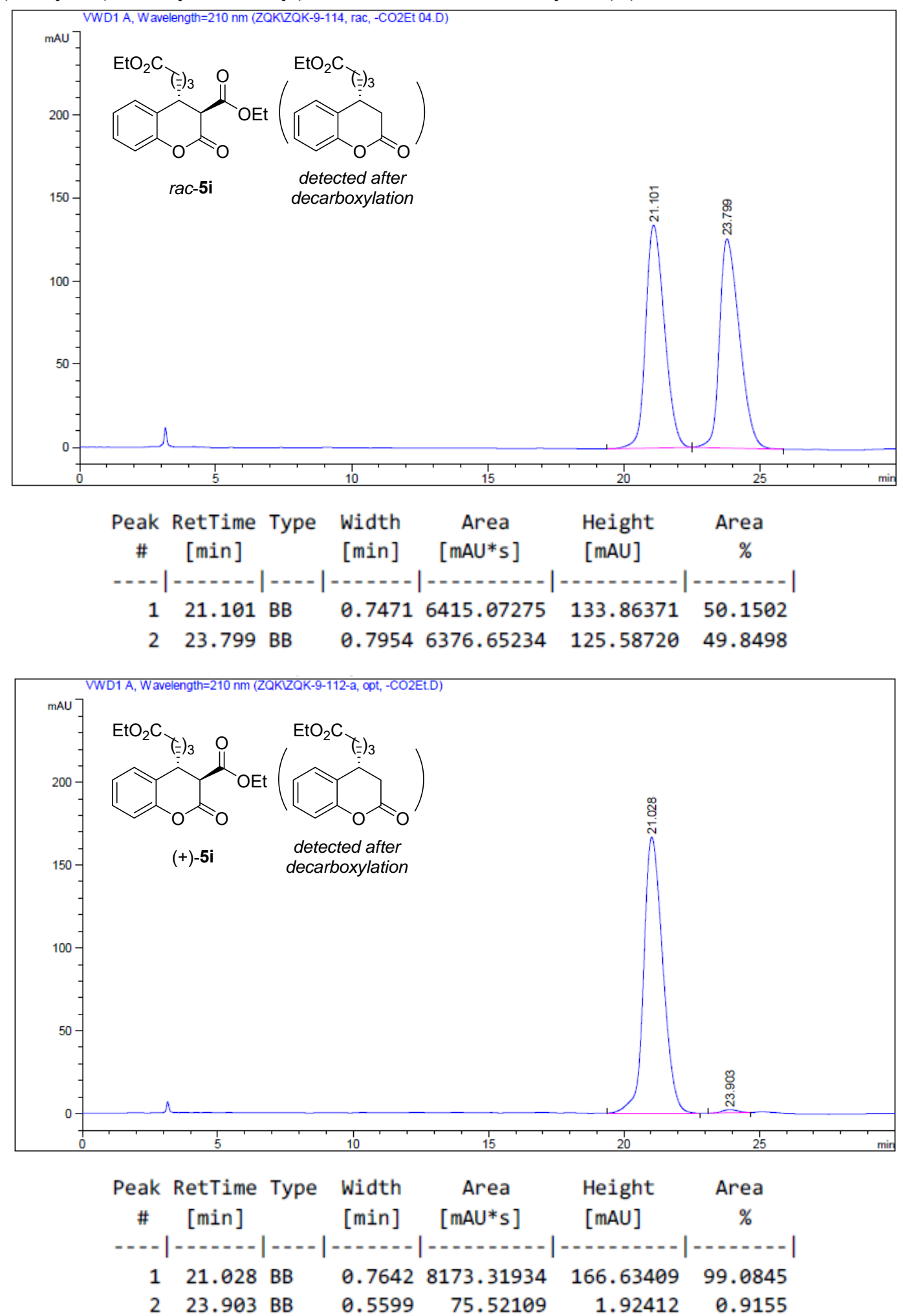
(+)-Ethyl-4-(2-((tert-butoxycarbonyl)(methyl)amino)ethyl)-2-oxochromane-3-carboxylate (5j)
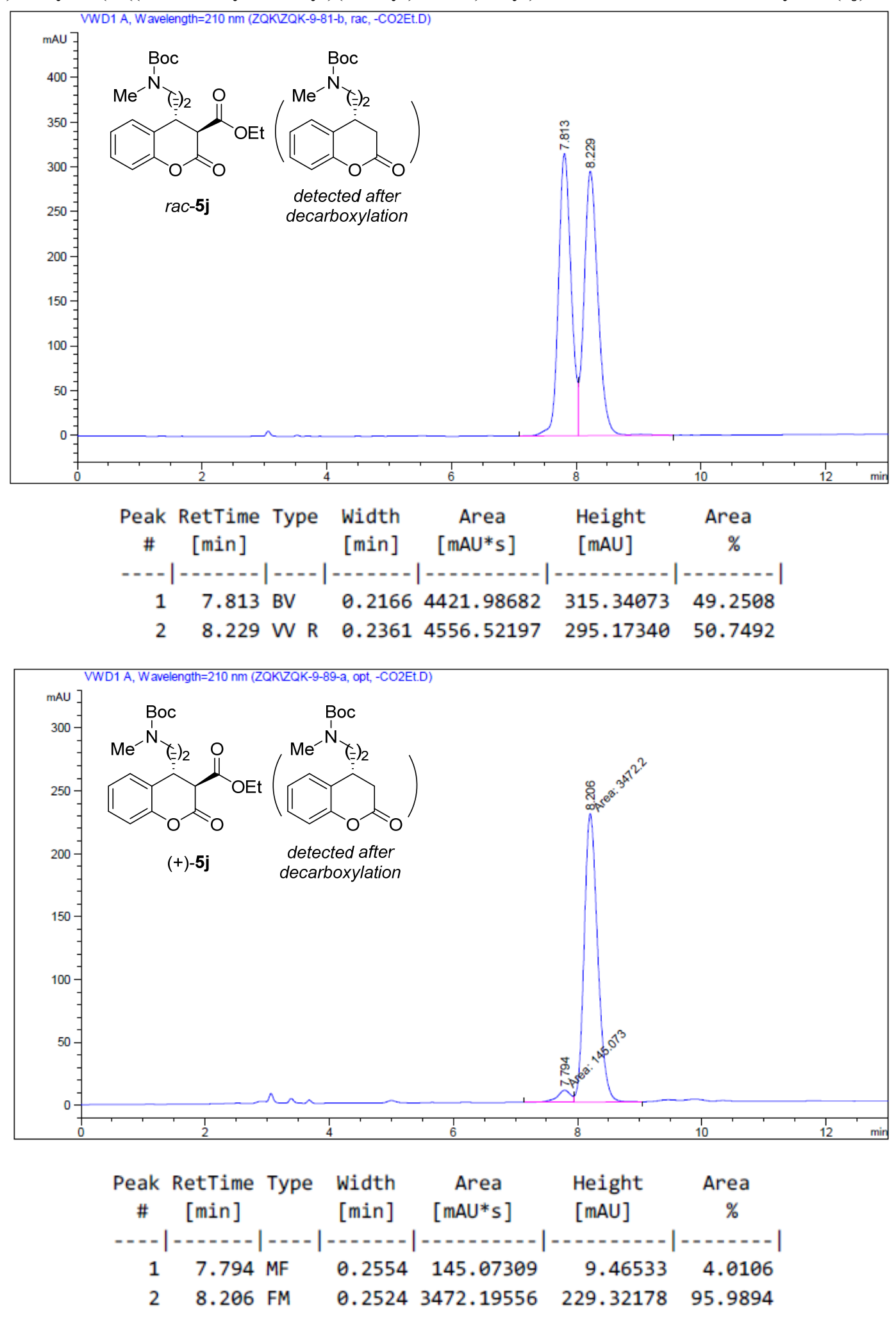
(-)-(3R,4S)-Ethyl-7-methoxy-2-oxo-4-pentylchromane-3-carboxylate ((3R,4S)-5k)
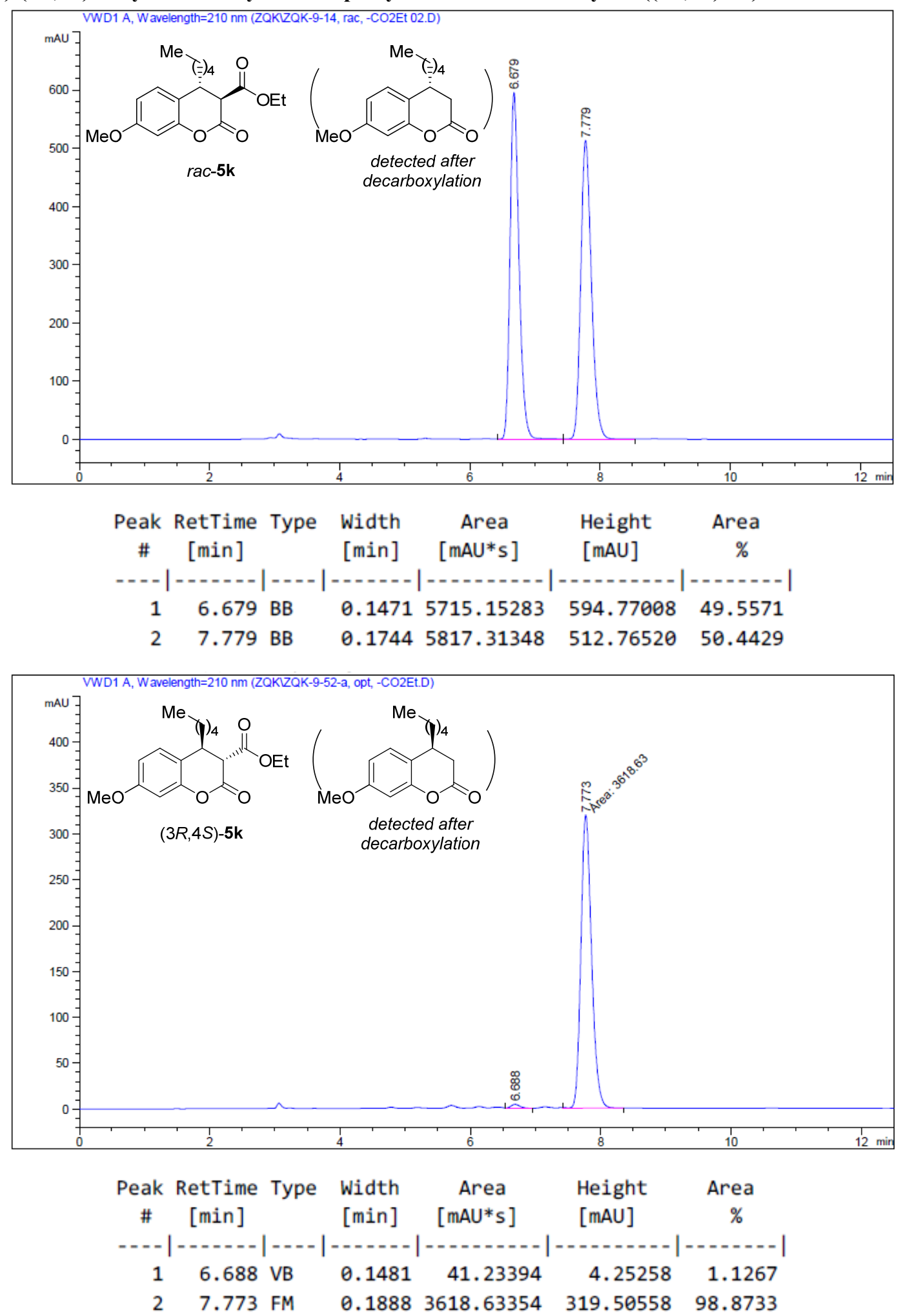
(+)-Ethyl-4-cyclopropyl-2-oxochromane-3-carboxylate (5l)
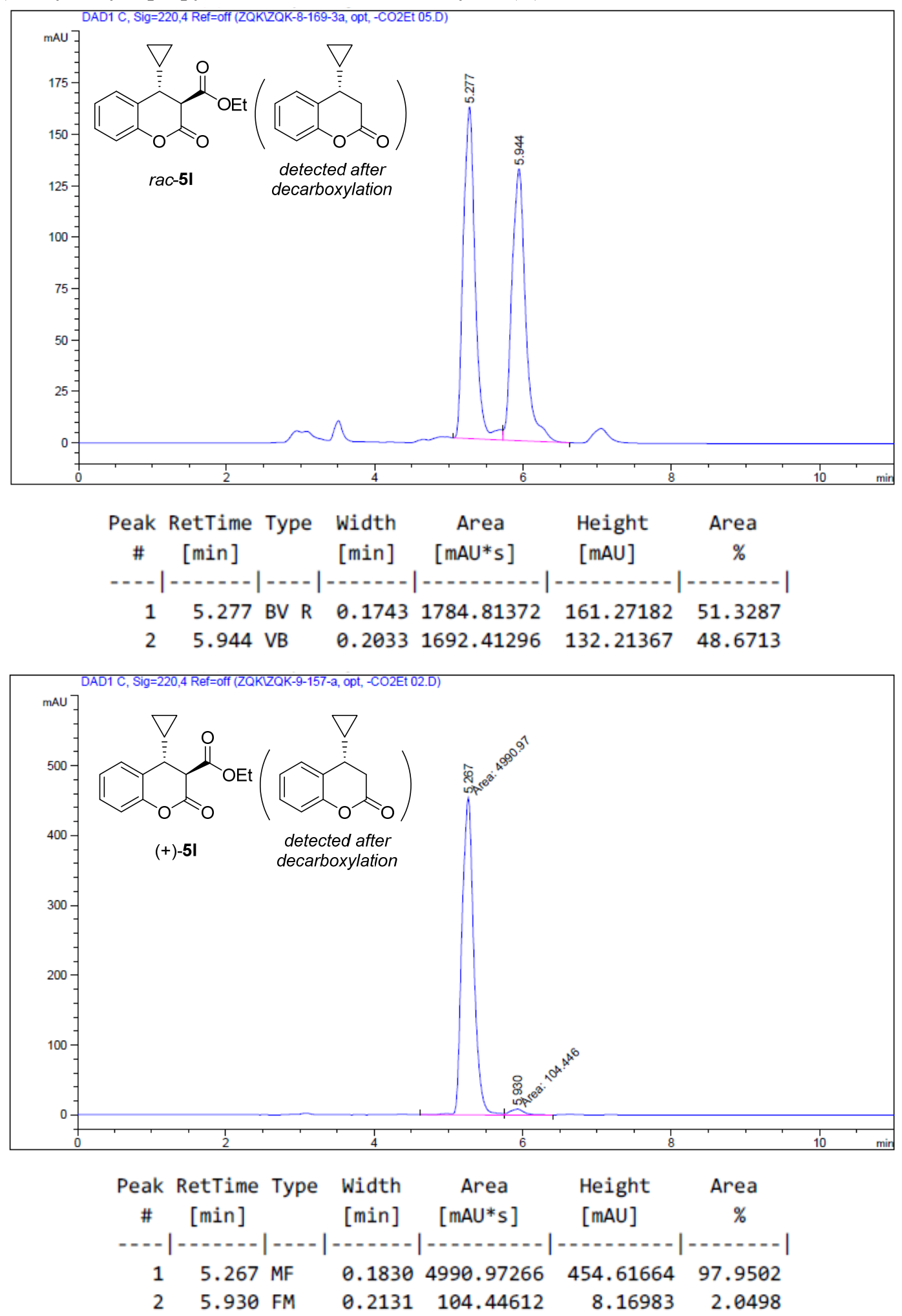
(+)-Ethyl-4-cyclopropyl-7-methoxy-2-oxochromane-3-carboxylate (5m)
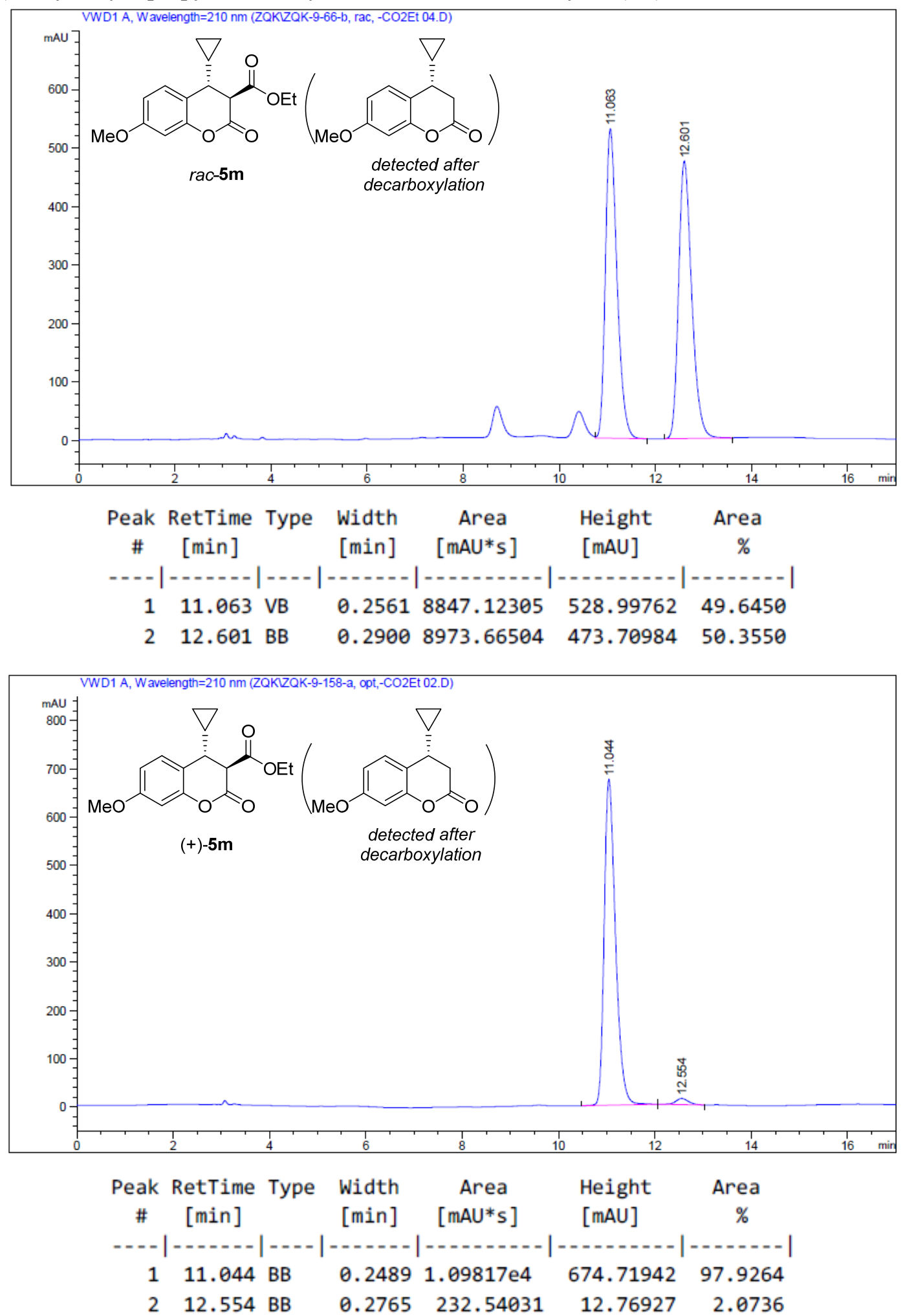
(+)-Ethyl-4-cyclopropyl-6-methoxy-2-oxochromane-3-carboxylate (5n)
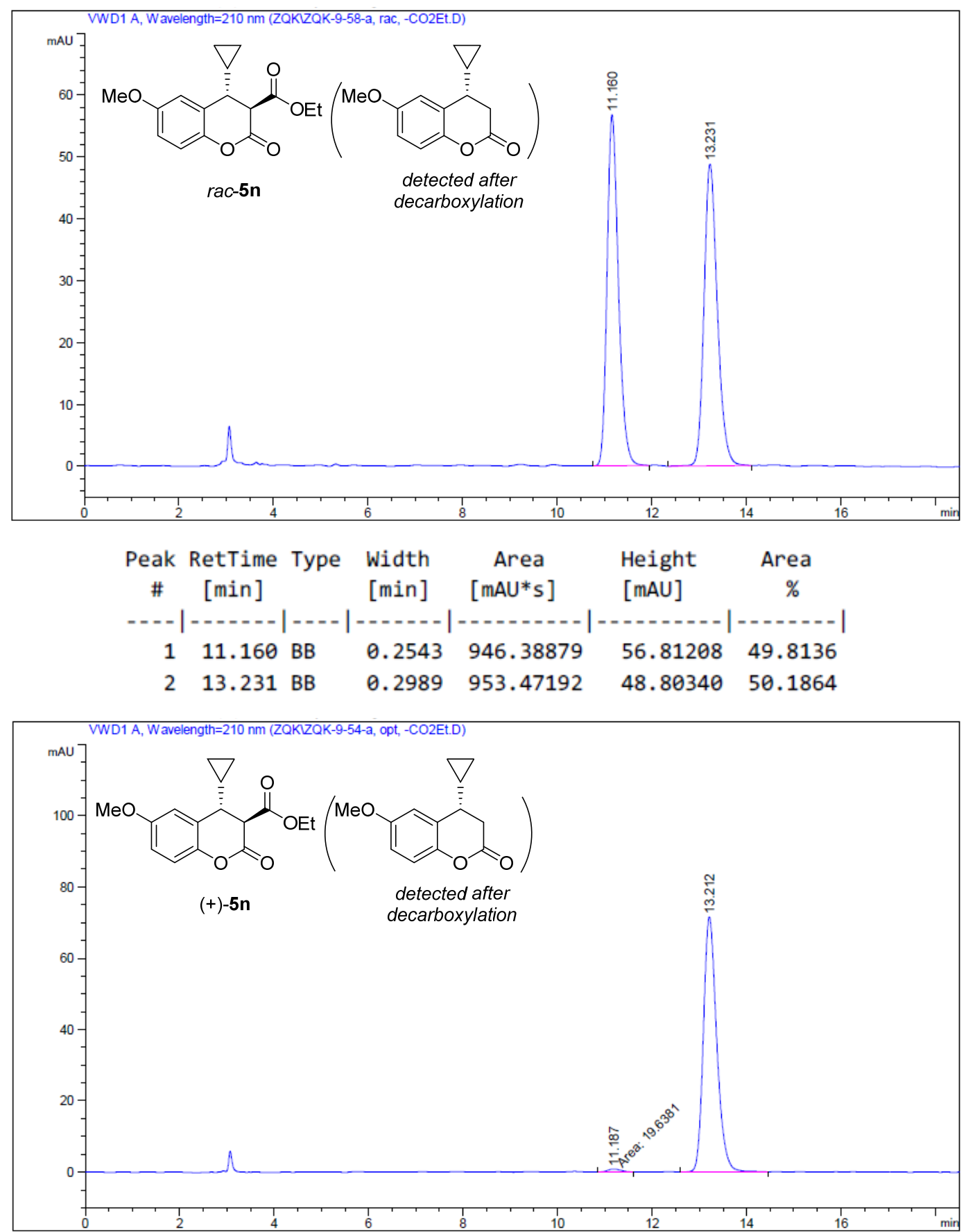
Peak RetTime Type Width Area Height Area \# $[\mathrm{min}] \quad[\mathrm{min}] \quad[\mathrm{mAU} * \mathrm{~s}] \quad[\mathrm{mAU}] \quad \%$

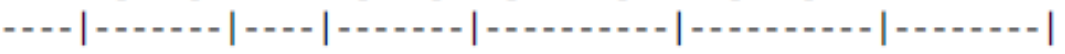
$111.187 \mathrm{MM}$
$0.3574 \quad 19.638119 .15913 \mathrm{e}-1$
1.3791
$213.212 \mathrm{BB}$
0.29961404 .32068
71.6546398 .6209 
(-)-(3R,4R)-Ethyl-2-oxo-4-phenylchromane-3-carboxylate $((3 R, 4 R)-50)$
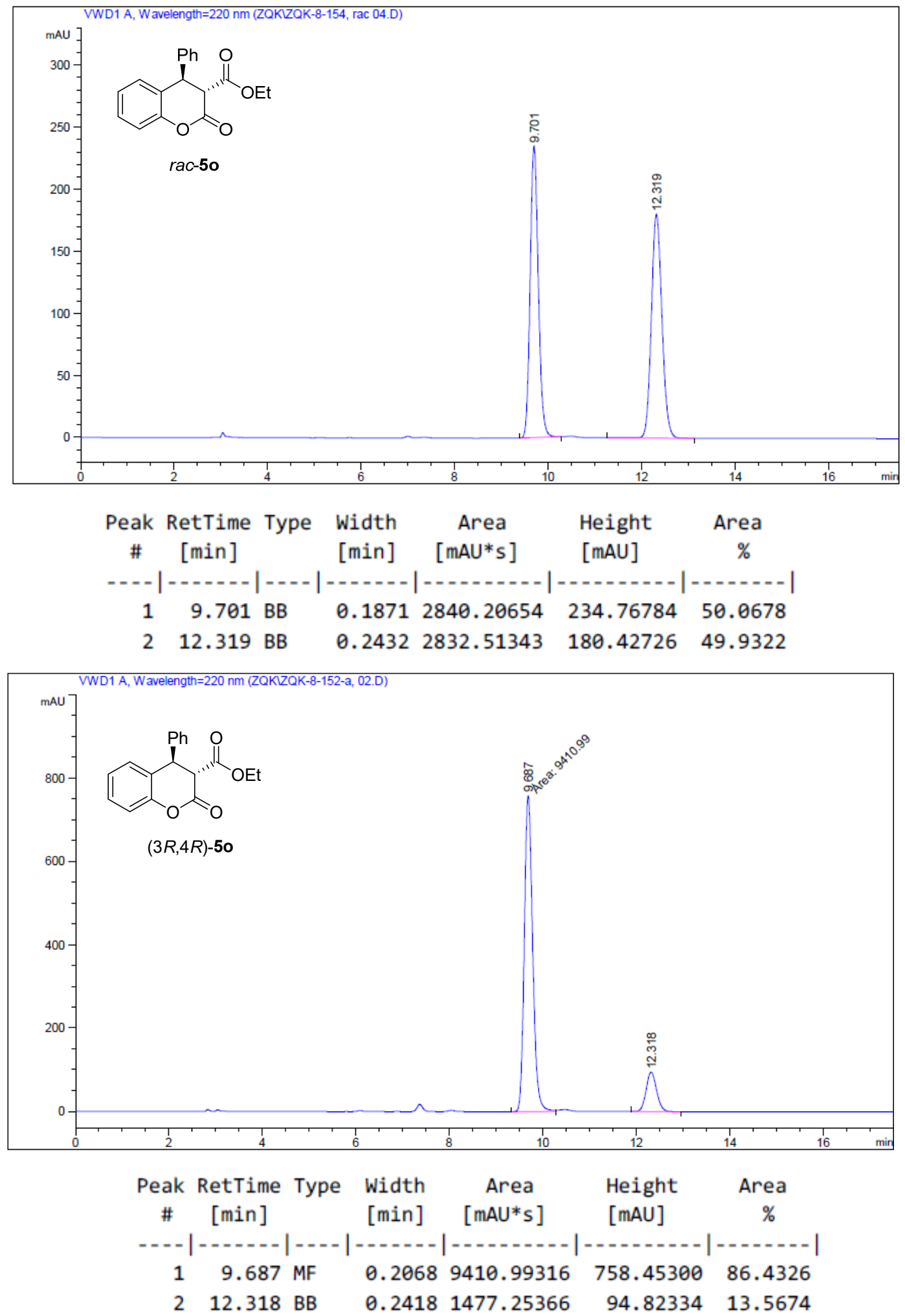
(G). Cartesian Coordinates and Energies of the Optimized Structures.

\section{TS-RR (entry 1):}

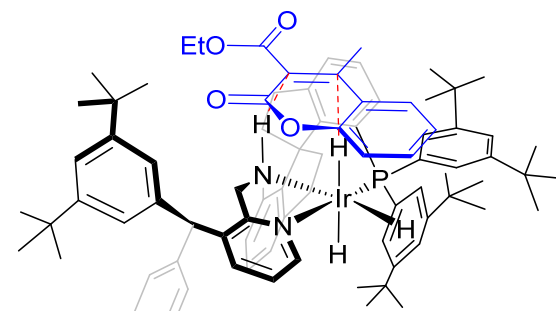

TS-RR

SCF energy [B3LYP-GD3BJ/6-311+G**/CPCM (MeOH)]: -4470.10756959 a.u.

Thermal correction to Gibbs free energy at 298 K: 1.77138000 a.u.

Gibbs free energy at $298 \mathrm{~K}$ [B3LYP-GD3BJ/6-311+G**/CPCM $(\mathrm{MeOH})]$ :

-4468.33618959 a.u.

\begin{tabular}{|c|c|c|c|}
\hline $\mathrm{Ir}$ & 0.924723 & -0.89194 & -0.50545 \\
\hline $\mathrm{P}$ & 3.086738 & -0.49355 & 0.095642 \\
\hline $\mathrm{C}$ & 2.250029 & 1.21113 & 3.093976 \\
\hline $\mathrm{C}$ & 1.437453 & 1.117834 & 4.410162 \\
\hline $\mathrm{C}$ & 2.171448 & 0.072753 & 5.267492 \\
\hline $\mathrm{C}$ & 2.931994 & -0.74417 & 4.251297 \\
\hline $\mathrm{C}$ & 2.838105 & -0.19916 & 2.955295 \\
\hline $\mathrm{C}$ & 3.467442 & -0.86027 & 1.87335 \\
\hline $\mathrm{C}$ & 4.255325 & -1.9899 & 2.163814 \\
\hline $\mathrm{C}$ & 4.377774 & -2.49141 & 3.461672 \\
\hline $\mathrm{C}$ & 3.686398 & -1.88782 & 4.510762 \\
\hline $\mathrm{C}$ & 3.484467 & 2.139376 & 3.349562 \\
\hline $\mathrm{C}$ & 3.012458 & 3.548466 & 3.020166 \\
\hline $\mathrm{C}$ & 1.959252 & 3.305521 & 1.973387 \\
\hline $\mathrm{C}$ & 1.487215 & 1.966702 & 1.998271 \\
\hline $\mathrm{C}$ & 0.425495 & 1.61363 & 1.132935 \\
\hline $\mathrm{C}$ & 0.04235 & 2.587954 & 0.172185 \\
\hline $\mathrm{C}$ & 0.534101 & 3.882904 & 0.160638 \\
\hline $\mathrm{C}$ & 1.489372 & 4.271296 & 1.09732 \\
\hline $\mathrm{N}$ & -0.26849 & 0.350079 & 1.041115 \\
\hline $\mathrm{C}$ & -1.66772 & 0.463005 & 0.595518 \\
\hline $\mathrm{C}$ & -2.04677 & -0.45457 & -0.53189 \\
\hline $\mathrm{C}$ & -3.39703 & -0.56822 & -0.91626 \\
\hline $\mathrm{C}$ & -3.70466 & -1.40397 & -1.98868 \\
\hline $\mathrm{C}$ & -2.683 & -2.0952 & -2.64387 \\
\hline $\mathrm{C}$ & -1.37993 & -1.94879 & -2.19743 \\
\hline $\mathrm{N}$ & -1.0727 & -1.14415 & -1.1603 \\
\hline $\mathrm{C}$ & 4.274446 & -1.56626 & -0.84088 \\
\hline
\end{tabular}




\begin{tabular}{|c|c|c|c|}
\hline C & 5.443477 & -1.07108 & -1.42632 \\
\hline $\mathrm{C}$ & 6.284402 & -1.89874 & -2.18575 \\
\hline $\mathrm{C}$ & 5.933647 & -3.24865 & -2.32338 \\
\hline $\mathrm{C}$ & 4.772881 & -3.78091 & -1.74165 \\
\hline $\mathrm{C}$ & 3.948322 & -2.91697 & -1.01333 \\
\hline $\mathrm{C}$ & 7.572621 & -1.32506 & -2.79907 \\
\hline $\mathrm{C}$ & 8.637791 & -1.23689 & -1.68529 \\
\hline $\mathrm{C}$ & 7.318666 & 0.082632 & -3.37656 \\
\hline $\mathrm{C}$ & 8.115102 & -2.21268 & -3.93193 \\
\hline $\mathrm{C}$ & 4.353117 & -5.24839 & -1.91826 \\
\hline $\mathrm{C}$ & 4.11304 & -5.88708 & -0.53456 \\
\hline $\mathrm{C}$ & 5.419626 & -6.07777 & -2.64939 \\
\hline $\mathrm{C}$ & 3.049905 & -5.28811 & -2.74488 \\
\hline $\mathrm{C}$ & 3.810042 & 1.17293 & -0.19598 \\
\hline $\mathrm{C}$ & 3.259875 & 2.002282 & -1.17924 \\
\hline $\mathrm{C}$ & 3.897058 & 3.190875 & -1.54616 \\
\hline $\mathrm{C}$ & 5.084403 & 3.539822 & -0.87968 \\
\hline $\mathrm{C}$ & 5.663756 & 2.731332 & 0.10633 \\
\hline $\mathrm{C}$ & 5.006841 & 1.533676 & 0.431893 \\
\hline $\mathrm{C}$ & 3.374361 & 4.0825 & -2.68301 \\
\hline $\mathrm{C}$ & 2.064153 & 3.5407 & -3.27428 \\
\hline $\mathrm{C}$ & 3.125895 & 5.512389 & -2.16164 \\
\hline $\mathrm{C}$ & 4.43371 & 4.1232 & -3.80553 \\
\hline $\mathrm{C}$ & 7.004817 & 3.077849 & 0.777241 \\
\hline $\mathrm{C}$ & 6.852424 & 3.0791 & 2.311257 \\
\hline $\mathrm{C}$ & 7.528637 & 4.456469 & 0.346735 \\
\hline $\mathrm{C}$ & 8.046399 & 2.013116 & 0.375752 \\
\hline $\mathrm{H}$ & 1.121883 & 0.194075 & -1.78401 \\
\hline $\mathrm{H}$ & 1.509559 & -1.92271 & -1.53951 \\
\hline $\mathrm{H}$ & 1.354721 & 2.102236 & 4.893789 \\
\hline $\mathrm{H}$ & 0.425855 & 0.788739 & 4.170056 \\
\hline $\mathrm{H}$ & 2.867684 & 0.541521 & 5.984313 \\
\hline $\mathrm{H}$ & 1.480322 & -0.561 & 5.841686 \\
\hline $\mathrm{H}$ & 4.764008 & -2.50935 & 1.353209 \\
\hline $\mathrm{H}$ & 4.994033 & -3.37557 & 3.641112 \\
\hline $\mathrm{H}$ & 3.737223 & -2.29479 & 5.523622 \\
\hline $\mathrm{H}$ & 3.870862 & 2.017796 & 4.370946 \\
\hline $\mathrm{H}$ & 4.283528 & 1.859228 & 2.659576 \\
\hline $\mathrm{H}$ & 2.568064 & 4.049595 & 3.898855 \\
\hline $\mathrm{H}$ & 3.82088 & 4.199241 & 2.6532 \\
\hline $\mathrm{H}$ & -0.63983 & 2.313645 & -0.62687 \\
\hline $\mathrm{H}$ & 0.170824 & 4.578613 & -0.59777 \\
\hline $\mathrm{H}$ & 1.887332 & 5.287574 & 1.124274 \\
\hline $\mathrm{H}$ & -0.32663 & -0.40042 & 1.724046 \\
\hline
\end{tabular}




\begin{tabular}{|c|c|c|c|}
\hline $\mathrm{H}$ & -1.89651 & 1.484206 & 0.291594 \\
\hline $\mathrm{H}$ & -2.32123 & 0.290872 & 1.458318 \\
\hline $\mathrm{H}$ & -4.74349 & -1.51729 & -2.30159 \\
\hline $\mathrm{H}$ & 5.694606 & -0.02133 & -1.30483 \\
\hline $\mathrm{H}$ & 6.575997 & -3.90208 & -2.90702 \\
\hline $\mathrm{H}$ & 3.017432 & -3.28261 & -0.58473 \\
\hline $\mathrm{H}$ & 9.578024 & -0.81281 & -2.07517 \\
\hline $\mathrm{H}$ & 8.85477 & -2.23461 & -1.27291 \\
\hline $\mathrm{H}$ & 8.292305 & -0.59956 & -0.85941 \\
\hline $\mathrm{H}$ & 6.525965 & 0.059179 & -4.14061 \\
\hline $\mathrm{H}$ & 8.237153 & 0.468933 & -3.84619 \\
\hline $\mathrm{H}$ & 7.017322 & 0.804859 & -2.60464 \\
\hline $\mathrm{H}$ & 7.364161 & -2.3592 & -4.72395 \\
\hline $\mathrm{H}$ & 8.428648 & -3.20309 & -3.5695 \\
\hline $\mathrm{H}$ & 8.999456 & -1.73902 & -4.38562 \\
\hline $\mathrm{H}$ & 5.034007 & -5.87155 & 0.06906 \\
\hline $\mathrm{H}$ & 3.79147 & -6.93534 & -0.64342 \\
\hline $\mathrm{H}$ & 3.335281 & -5.35854 & 0.034802 \\
\hline $\mathrm{H}$ & 6.378992 & -6.0749 & -2.10901 \\
\hline $\mathrm{H}$ & 5.599263 & -5.70686 & -3.66989 \\
\hline $\mathrm{H}$ & 5.08542 & -7.12354 & -2.73259 \\
\hline $\mathrm{H}$ & 2.698874 & -6.32639 & -2.86596 \\
\hline $\mathrm{H}$ & 3.213764 & -4.86247 & -3.74702 \\
\hline $\mathrm{H}$ & 2.247878 & -4.70764 & -2.26797 \\
\hline $\mathrm{H}$ & 2.33599 & 1.676053 & -1.65353 \\
\hline $\mathrm{H}$ & 5.580356 & 4.466994 & -1.16091 \\
\hline $\mathrm{H}$ & 5.445944 & 0.852111 & 1.162478 \\
\hline $\mathrm{H}$ & 1.702932 & 4.215427 & -4.06586 \\
\hline $\mathrm{H}$ & 1.279389 & 3.463549 & -2.51031 \\
\hline $\mathrm{H}$ & 2.19866 & 2.54287 & -3.71749 \\
\hline $\mathrm{H}$ & 2.76108 & 6.15914 & -2.97599 \\
\hline $\mathrm{H}$ & 4.044128 & 5.967612 & -1.76068 \\
\hline $\mathrm{H}$ & 4.639042 & 3.109715 & -4.18428 \\
\hline $\mathrm{H}$ & 5.385189 & 4.550003 & -3.45352 \\
\hline $\mathrm{H}$ & 4.079052 & 4.74005 & -4.64741 \\
\hline $\mathrm{H}$ & 6.108488 & 3.822613 & 2.634413 \\
\hline $\mathrm{H}$ & 7.813498 & 3.327936 & 2.788954 \\
\hline $\mathrm{H}$ & 6.537481 & 2.098118 & 2.694704 \\
\hline $\mathrm{H}$ & 7.722077 & 4.502548 & -0.73569 \\
\hline $\mathrm{H}$ & 8.477819 & 4.671303 & 0.861369 \\
\hline $\mathrm{H}$ & 6.819636 & 5.258843 & 0.60311 \\
\hline $\mathrm{H}$ & 7.739102 & 1.008331 & 0.70154 \\
\hline $\mathrm{H}$ & 9.023375 & 2.231663 & 0.836739 \\
\hline $\mathrm{H}$ & 8.181155 & 1.98638 & -0.71628 \\
\hline
\end{tabular}




\begin{tabular}{|c|c|c|c|}
\hline $\mathrm{H}$ & 2.374094 & 5.509315 & -1.3601 \\
\hline $\mathrm{H}$ & 0.802272 & -1.95964 & 0.423058 \\
\hline $\mathrm{H}$ & -2.89564 & -2.76108 & -3.48098 \\
\hline $\mathrm{H}$ & -0.54471 & -2.48287 & -2.64701 \\
\hline $\mathrm{C}$ & -4.44826 & 0.229947 & -0.1527 \\
\hline $\mathrm{H}$ & -4.23898 & 0.058082 & 0.91311 \\
\hline $\mathrm{C}$ & -5.84454 & -0.31808 & -0.37929 \\
\hline $\mathrm{C}$ & -6.18076 & -1.4977 & 0.293227 \\
\hline $\mathrm{C}$ & -6.75636 & 0.253586 & -1.26524 \\
\hline $\mathrm{C}$ & -7.40827 & -2.13032 & 0.077089 \\
\hline $\mathrm{H}$ & -5.4498 & -1.91887 & 0.986477 \\
\hline $\mathrm{C}$ & -8.0045 & -0.34526 & -1.50248 \\
\hline $\mathrm{H}$ & -6.48541 & 1.179412 & -1.77426 \\
\hline $\mathrm{C}$ & -8.30618 & -1.53654 & -0.82634 \\
\hline $\mathrm{H}$ & -9.26568 & -2.01409 & -1.00357 \\
\hline $\mathrm{C}$ & -4.26004 & 1.71994 & -0.39965 \\
\hline $\mathrm{C}$ & -4.30971 & 2.60309 & 0.683333 \\
\hline $\mathrm{C}$ & -3.91335 & 2.203317 & -1.66561 \\
\hline $\mathrm{C}$ & -3.97577 & 3.954652 & 0.534571 \\
\hline $\mathrm{H}$ & -4.56121 & 2.198913 & 1.663595 \\
\hline $\mathrm{C}$ & -3.58645 & 3.55179 & -1.86149 \\
\hline $\mathrm{H}$ & -3.85748 & 1.4989 & -2.49338 \\
\hline $\mathrm{C}$ & -3.61775 & 4.400796 & -0.74628 \\
\hline $\mathrm{H}$ & -3.33057 & 5.445113 & -0.87504 \\
\hline $\mathrm{C}$ & -7.71223 & -3.43456 & 0.82951 \\
\hline $\mathrm{C}$ & -6.62101 & -4.47495 & 0.494362 \\
\hline $\mathrm{C}$ & -7.69584 & -3.15536 & 2.347576 \\
\hline $\mathrm{C}$ & -9.08026 & -4.02117 & 0.452231 \\
\hline $\mathrm{H}$ & -6.59578 & -4.68052 & -0.58801 \\
\hline $\mathrm{H}$ & -5.62371 & -4.12558 & 0.79881 \\
\hline $\mathrm{H}$ & -6.82 & -5.4239 & 1.018534 \\
\hline $\mathrm{H}$ & -8.45927 & -2.40816 & 2.615773 \\
\hline $\mathrm{H}$ & -7.90619 & -4.07919 & 2.911073 \\
\hline $\mathrm{H}$ & -6.71823 & -2.77357 & 2.677161 \\
\hline $\mathrm{H}$ & -9.25217 & -4.95571 & 1.008462 \\
\hline $\mathrm{H}$ & -9.90239 & -3.33182 & 0.699223 \\
\hline $\mathrm{H}$ & -9.14175 & -4.25627 & -0.6218 \\
\hline $\mathrm{C}$ & -8.97963 & 0.32153 & -2.48583 \\
\hline $\mathrm{C}$ & -9.32553 & 1.736062 & -1.97435 \\
\hline $\mathrm{C}$ & -8.31046 & 0.427358 & -3.87279 \\
\hline $\mathrm{C}$ & -10.2868 & -0.47033 & -2.64069 \\
\hline $\mathrm{H}$ & -9.80319 & 1.686673 & -0.98338 \\
\hline $\mathrm{H}$ & -8.42833 & 2.365983 & -1.88219 \\
\hline $\mathrm{H}$ & -10.021 & 2.237867 & -2.66695 \\
\hline
\end{tabular}




\begin{tabular}{|c|c|c|c|}
\hline $\mathrm{H}$ & -8.04842 & -0.57095 & -4.25753 \\
\hline $\mathrm{H}$ & -8.9919 & 0.905414 & -4.59537 \\
\hline $\mathrm{H}$ & -7.38819 & 1.026131 & -3.83524 \\
\hline $\mathrm{H}$ & -10.9511 & 0.042323 & -3.3536 \\
\hline $\mathrm{H}$ & -10.1061 & -1.486 & -3.02503 \\
\hline $\mathrm{H}$ & -10.8271 & -0.55674 & -1.6856 \\
\hline $\mathrm{C}$ & -3.90715 & 4.921374 & 1.726609 \\
\hline $\mathrm{C}$ & -4.45284 & 4.28753 & 3.015467 \\
\hline $\mathrm{C}$ & -2.42872 & 5.30299 & 1.958359 \\
\hline $\mathrm{C}$ & -4.73209 & 6.188749 & 1.425608 \\
\hline $\mathrm{H}$ & -5.50079 & 3.969972 & 2.90061 \\
\hline $\mathrm{H}$ & -3.86226 & 3.413121 & 3.324411 \\
\hline $\mathrm{H}$ & -4.41138 & 5.019823 & 3.836519 \\
\hline $\mathrm{H}$ & -1.99626 & 5.802265 & 1.078474 \\
\hline $\mathrm{H}$ & -2.33665 & 5.988374 & 2.81689 \\
\hline $\mathrm{H}$ & -1.81699 & 4.410225 & 2.158747 \\
\hline $\mathrm{H}$ & -4.6876 & 6.885119 & 2.278379 \\
\hline $\mathrm{H}$ & -4.35781 & 6.724604 & 0.540859 \\
\hline $\mathrm{H}$ & -5.7885 & 5.935598 & 1.244434 \\
\hline $\mathrm{C}$ & -3.15076 & 4.102836 & -3.22803 \\
\hline $\mathrm{C}$ & -1.69867 & 4.614588 & -3.12129 \\
\hline $\mathrm{C}$ & -3.20156 & 3.029916 & -4.32678 \\
\hline $\mathrm{C}$ & -4.08244 & 5.262248 & -3.63611 \\
\hline $\mathrm{H}$ & -1.60279 & 5.418299 & -2.37587 \\
\hline $\mathrm{H}$ & -1.01898 & 3.800171 & -2.8293 \\
\hline $\mathrm{H}$ & -1.35434 & 5.011861 & -4.08982 \\
\hline $\mathrm{H}$ & -4.21714 & 2.623971 & -4.45334 \\
\hline $\mathrm{H}$ & -2.89442 & 3.467574 & -5.28909 \\
\hline $\mathrm{H}$ & -2.52121 & 2.192019 & -4.11145 \\
\hline $\mathrm{H}$ & -3.78178 & 5.672213 & -4.61391 \\
\hline $\mathrm{H}$ & -5.12513 & 4.916609 & -3.71474 \\
\hline $\mathrm{H}$ & -4.05621 & 6.084439 & -2.90565 \\
\hline $\mathrm{C}$ & -0.34702 & -5.53082 & -1.00701 \\
\hline C & -1.7389 & -5.59751 & -1.17104 \\
\hline $\mathrm{C}$ & -2.55786 & -4.72794 & -0.46199 \\
\hline $\mathrm{C}$ & -1.97633 & -3.79469 & 0.404578 \\
\hline $\mathrm{C}$ & -0.5977 & -3.71337 & 0.603542 \\
\hline $\mathrm{C}$ & 0.205584 & -4.60429 & -0.1298 \\
\hline $\mathrm{C}$ & -0.05164 & -2.71319 & 1.567112 \\
\hline $\mathrm{C}$ & -0.93535 & -1.88155 & 2.207054 \\
\hline $\mathrm{C}$ & -0.73313 & -1.41691 & 3.616053 \\
\hline $\mathrm{C}$ & -2.39322 & -2.02122 & 1.976497 \\
\hline $\mathrm{O}$ & -2.82413 & -2.95839 & 1.056769 \\
\hline $\mathrm{O}$ & -0.12707 & -1.98515 & 4.497569 \\
\hline
\end{tabular}




$\begin{array}{llcl}\mathrm{C} & 1.183358 & -3.19669 & 2.299135 \\ \mathrm{O} & -3.30083 & -1.43793 & 2.538688 \\ \mathrm{O} & -1.38758 & -0.25398 & 3.829424 \\ \mathrm{C} & -2.69997 & 1.27398 & 5.071615 \\ \mathrm{C} & -1.80526 & 0.056749 & 5.162943 \\ \mathrm{H} & 0.306852 & -6.20172 & -1.56657 \\ \mathrm{H} & -2.17977 & -6.32572 & -1.85555 \\ \mathrm{H} & -3.64325 & -4.7348 & -0.5674 \\ \mathrm{H} & 1.28628 & -4.55241 & -0.02266 \\ \mathrm{H} & 1.938625 & -3.58645 & 1.611138 \\ \mathrm{H} & 1.633443 & -2.40826 & 2.895861 \\ \mathrm{H} & 0.881153 & -3.99676 & 2.993128 \\ \mathrm{H} & -3.07671 & 1.55094 & 6.068231 \\ \mathrm{H} & -2.14766 & 2.132528 & 4.659054 \\ \mathrm{H} & -3.55602 & 1.062214 & 4.414311 \\ \mathrm{H} & -2.33775 & -0.81613 & 5.572137 \\ \mathrm{H} & -0.92498 & 0.238783 & 5.799144\end{array}$

\section{TS-SS (entry 2):}

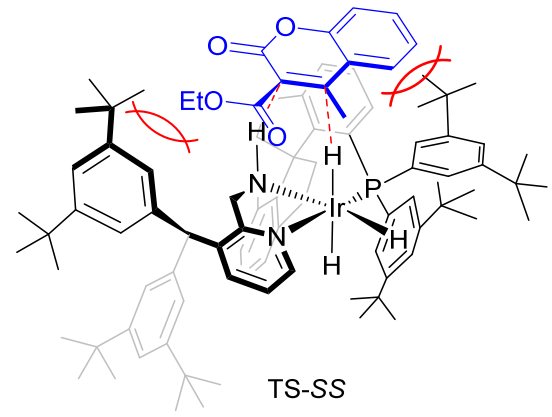

SCF energy [B3LYP-GD3BJ/6-311+G**/CPCM (MeOH)]: -4470.09852978 a.u. Thermal correction to Gibbs free energy at $298 \mathrm{~K}$ : 1.76711000 a.u.

Gibbs free energy at $298 \mathrm{~K}$ [B3LYP-GD3BJ/6-311+G**/CPCM $(\mathrm{MeOH})]$ : -4468.33141978 a.u.

$\begin{array}{cccc}01 & & & \\ \mathrm{Ir} & 0.885654 & -0.82243 & -0.81152 \\ \mathrm{P} & 2.96001 & -0.21097 & -0.14114 \\ \mathrm{C} & 2.013887 & 0.936123 & 3.072148 \\ \mathrm{C} & 1.384701 & 0.527976 & 4.432824 \\ \mathrm{C} & 2.492533 & -0.27259 & 5.134038 \\ \mathrm{C} & 3.279387 & -0.84415 & 3.977923 \\ \mathrm{C} & 2.884676 & -0.28892 & 2.741865 \\ \mathrm{C} & 3.493256 & -0.75053 & 1.548996 \\ \mathrm{C} & 4.516644 & -1.71145 & 1.657894 \\ \mathrm{C} & 4.932585 & -2.20701 & 2.894161 \\ \mathrm{C} & 4.299856 & -1.78759 & 4.062255 \\ & & \mathrm{~S} 134 & \end{array}$




\begin{tabular}{|c|c|c|c|}
\hline $\mathrm{C}$ & 3.019505 & 2.107086 & 3.339533 \\
\hline $\mathrm{C}$ & 2.19308 & 3.384458 & 3.2672 \\
\hline $\mathrm{C}$ & 1.084984 & 3.007633 & 2.321222 \\
\hline $\mathrm{C}$ & 0.979953 & 1.603181 & 2.1576 \\
\hline $\mathrm{C}$ & -0.05537 & 1.1045 & 1.337086 \\
\hline $\mathrm{C}$ & -0.85335 & 2.050323 & 0.646398 \\
\hline $\mathrm{C}$ & -0.72565 & 3.419264 & 0.827971 \\
\hline $\mathrm{C}$ & 0.244571 & 3.916398 & 1.694801 \\
\hline $\mathrm{N}$ & -0.34751 & -0.28154 & 1.070948 \\
\hline $\mathrm{C}$ & -1.76039 & -0.5129 & 0.711909 \\
\hline $\mathrm{C}$ & -2.07892 & -0.80656 & -0.73188 \\
\hline $\mathrm{C}$ & -3.41818 & -0.84768 & -1.16695 \\
\hline $\mathrm{C}$ & -3.66887 & -1.25542 & -2.47811 \\
\hline $\mathrm{C}$ & -2.60295 & -1.60406 & -3.31078 \\
\hline $\mathrm{C}$ & -1.31102 & -1.53114 & -2.81214 \\
\hline $\mathrm{N}$ & -1.06203 & -1.14112 & -1.54679 \\
\hline $\mathrm{C}$ & 4.247529 & -0.83775 & -1.31757 \\
\hline $\mathrm{C}$ & 5.041036 & 0.056297 & -2.05038 \\
\hline $\mathrm{C}$ & 5.929709 & -0.39316 & -3.03057 \\
\hline $\mathrm{C}$ & 6.021973 & -1.77576 & -3.2506 \\
\hline $\mathrm{C}$ & 5.233943 & -2.69755 & -2.5505 \\
\hline $\mathrm{C}$ & 4.331974 & -2.20728 & -1.59163 \\
\hline $\mathrm{C}$ & 6.808565 & 0.583013 & -3.82917 \\
\hline $\mathrm{C}$ & 8.267099 & 0.438215 & -3.34524 \\
\hline $\mathrm{C}$ & 6.368094 & 2.043949 & -3.63362 \\
\hline $\mathrm{C}$ & 6.730405 & 0.259449 & -5.3348 \\
\hline $\mathrm{C}$ & 5.323366 & -4.21028 & -2.80154 \\
\hline $\mathrm{C}$ & 5.807613 & -4.89336 & -1.50612 \\
\hline $\mathrm{C}$ & 6.305143 & -4.56315 & -3.92916 \\
\hline $\mathrm{C}$ & 3.928609 & -4.73932 & -3.19559 \\
\hline $\mathrm{C}$ & 3.387442 & 1.582699 & -0.13513 \\
\hline $\mathrm{C}$ & 2.614286 & 2.509421 & -0.84003 \\
\hline $\mathrm{C}$ & 3.052294 & 3.826743 & -1.0057 \\
\hline $\mathrm{C}$ & 4.271812 & 4.199887 & -0.41319 \\
\hline $\mathrm{C}$ & 5.06466 & 3.298672 & 0.308649 \\
\hline $\mathrm{C}$ & 4.602878 & 1.979431 & 0.429662 \\
\hline $\mathrm{C}$ & 2.273457 & 4.845292 & -1.85232 \\
\hline $\mathrm{C}$ & 0.98182 & 4.241261 & -2.42528 \\
\hline $\mathrm{C}$ & 1.902299 & 6.068432 & -0.99006 \\
\hline $\mathrm{C}$ & 3.163391 & 5.295027 & -3.03081 \\
\hline $\mathrm{C}$ & 6.411595 & 3.687757 & 0.940222 \\
\hline $\mathrm{C}$ & 6.361973 & 3.421266 & 2.459253 \\
\hline $\mathrm{C}$ & 6.747936 & 5.17023 & 0.720661 \\
\hline $\mathrm{C}$ & 7.533144 & 2.836171 & 0.308235 \\
\hline
\end{tabular}




\begin{tabular}{|c|c|c|c|}
\hline $\mathrm{H}$ & 0.845005 & 0.549775 & -1.69427 \\
\hline $\mathrm{H}$ & 1.579442 & -1.40097 & -2.10217 \\
\hline $\mathrm{H}$ & 1.053024 & 1.411968 & 4.997502 \\
\hline $\mathrm{H}$ & 0.510486 & -0.11093 & 4.262121 \\
\hline $\mathrm{H}$ & 3.135503 & 0.371895 & 5.758723 \\
\hline $\mathrm{H}$ & 2.090898 & -1.0701 & 5.77235 \\
\hline $\mathrm{H}$ & 5.007927 & -2.07626 & 0.75783 \\
\hline $\mathrm{H}$ & 5.738461 & -2.94347 & 2.93248 \\
\hline $\mathrm{H}$ & 4.585966 & -2.19866 & 5.033403 \\
\hline $\mathrm{H}$ & 3.552181 & 1.972593 & 4.289608 \\
\hline $\mathrm{H}$ & 3.766457 & 2.117792 & 2.543584 \\
\hline $\mathrm{H}$ & 1.776385 & 3.663372 & 4.251638 \\
\hline $\mathrm{H}$ & 2.770539 & 4.249584 & 2.904777 \\
\hline $\mathrm{H}$ & -1.58903 & 1.714382 & -0.07855 \\
\hline $\mathrm{H}$ & -1.39171 & 4.088235 & 0.284293 \\
\hline $\mathrm{H}$ & 0.357925 & 4.98797 & 1.871156 \\
\hline $\mathrm{H}$ & -0.06783 & -1.02801 & 1.701665 \\
\hline $\mathrm{H}$ & -2.37668 & 0.311964 & 1.079694 \\
\hline $\mathrm{H}$ & -2.13168 & -1.38938 & 1.244391 \\
\hline $\mathrm{H}$ & -4.69667 & -1.30159 & -2.84065 \\
\hline $\mathrm{H}$ & 4.947977 & 1.119778 & -1.86521 \\
\hline $\mathrm{H}$ & 6.722361 & -2.13915 & -3.99987 \\
\hline $\mathrm{H}$ & 3.671582 & -2.89527 & -1.06054 \\
\hline $\mathrm{H}$ & 8.93248 & 1.118557 & -3.90215 \\
\hline $\mathrm{H}$ & 8.635452 & -0.58957 & -3.48657 \\
\hline $\mathrm{H}$ & 8.346946 & 0.67961 & -2.27382 \\
\hline $\mathrm{H}$ & 5.321176 & 2.195846 & -3.93858 \\
\hline $\mathrm{H}$ & 6.999052 & 2.7079 & -4.2447 \\
\hline $\mathrm{H}$ & 6.46181 & 2.369106 & -2.58789 \\
\hline $\mathrm{H}$ & 5.692477 & 0.326644 & -5.69658 \\
\hline $\mathrm{H}$ & 7.102234 & -0.75037 & -5.56227 \\
\hline $\mathrm{H}$ & 7.341682 & 0.973044 & -5.9104 \\
\hline $\mathrm{H}$ & 6.816936 & -4.54186 & -1.23946 \\
\hline $\mathrm{H}$ & 5.848219 & -5.98791 & -1.63394 \\
\hline $\mathrm{H}$ & 5.140092 & -4.67906 & -0.66173 \\
\hline $\mathrm{H}$ & 7.332218 & -4.24403 & -3.69431 \\
\hline $\mathrm{H}$ & 6.012293 & -4.1034 & -4.88573 \\
\hline $\mathrm{H}$ & 6.323557 & -5.65439 & -4.07547 \\
\hline $\mathrm{H}$ & 3.957759 & -5.83165 & -3.34222 \\
\hline $\mathrm{H}$ & 3.59185 & -4.27542 & -4.13592 \\
\hline $\mathrm{H}$ & 3.176863 & -4.52065 & -2.42625 \\
\hline $\mathrm{H}$ & 1.678357 & 2.162774 & -1.27181 \\
\hline $\mathrm{H}$ & 4.61549 & 5.225454 & -0.53716 \\
\hline $\mathrm{H}$ & 5.208756 & 1.237102 & 0.9521 \\
\hline
\end{tabular}




\begin{tabular}{|c|c|c|c|}
\hline $\mathrm{H}$ & 0.442629 & 5.001765 & -3.01118 \\
\hline $\mathrm{H}$ & 0.314659 & 3.888373 & -1.62702 \\
\hline $\mathrm{H}$ & 1.190519 & 3.389795 & -3.09019 \\
\hline $\mathrm{H}$ & 1.353548 & 6.811248 & -1.59175 \\
\hline $\mathrm{H}$ & 2.794467 & 6.564616 & -0.57878 \\
\hline $\mathrm{H}$ & 3.45322 & 4.432578 & -3.65148 \\
\hline $\mathrm{H}$ & 4.086346 & 5.783374 & -2.68337 \\
\hline $\mathrm{H}$ & 2.623065 & 6.012769 & -3.66951 \\
\hline $\mathrm{H}$ & 5.561354 & 4.008113 & 2.935296 \\
\hline $\mathrm{H}$ & 7.318621 & 3.701811 & 2.928727 \\
\hline $\mathrm{H}$ & 6.178114 & 2.360588 & 2.683725 \\
\hline $\mathrm{H}$ & 6.828121 & 5.416964 & -0.34914 \\
\hline $\mathrm{H}$ & 7.715795 & 5.406399 & 1.18925 \\
\hline $\mathrm{H}$ & 5.990157 & 5.830889 & 1.169458 \\
\hline $\mathrm{H}$ & 7.351649 & 1.759111 & 0.439186 \\
\hline $\mathrm{H}$ & 8.504836 & 3.073756 & 0.770725 \\
\hline $\mathrm{H}$ & 7.613271 & 3.0334 & -0.77179 \\
\hline $\mathrm{H}$ & 1.264233 & 5.766189 & -0.14759 \\
\hline $\mathrm{H}$ & 0.886471 & -2.32356 & 0.009377 \\
\hline $\mathrm{H}$ & -2.77078 & -1.92804 & -4.33898 \\
\hline $\mathrm{H}$ & -0.43545 & -1.77816 & -3.41135 \\
\hline $\mathrm{C}$ & -4.52915 & -0.39646 & -0.2229 \\
\hline $\mathrm{H}$ & -4.34319 & -0.87198 & 0.752683 \\
\hline $\mathrm{C}$ & -5.90254 & -0.88244 & -0.66995 \\
\hline $\mathrm{C}$ & -6.23012 & -2.22938 & -0.47889 \\
\hline $\mathrm{C}$ & -6.82086 & -0.04317 & -1.30059 \\
\hline $\mathrm{C}$ & -7.45075 & -2.75391 & -0.91998 \\
\hline $\mathrm{H}$ & -5.50424 & -2.87644 & 0.019869 \\
\hline $\mathrm{C}$ & -8.05623 & -0.52629 & -1.75683 \\
\hline $\mathrm{H}$ & -6.56809 & 1.007636 & -1.43322 \\
\hline $\mathrm{C}$ & -8.34855 & -1.88233 & -1.55929 \\
\hline $\mathrm{H}$ & -9.30085 & -2.27179 & -1.90688 \\
\hline C & -4.45519 & 1.110905 & -0.01375 \\
\hline $\mathrm{C}$ & -4.64544 & 1.65087 & 1.260193 \\
\hline $\mathrm{C}$ & -4.20819 & 1.965118 & -1.09378 \\
\hline $\mathrm{C}$ & -4.57777 & 3.033367 & 1.479921 \\
\hline $\mathrm{H}$ & -4.83179 & 0.967762 & 2.088754 \\
\hline $\mathrm{C}$ & -4.17022 & 3.354823 & -0.92585 \\
\hline $\mathrm{H}$ & -4.04991 & 1.528029 & -2.07727 \\
\hline $\mathrm{C}$ & -4.34703 & 3.859678 & 0.370705 \\
\hline $\mathrm{H}$ & -4.29637 & 4.937766 & 0.523735 \\
\hline $\mathrm{C}$ & -7.75947 & -4.24271 & -0.69486 \\
\hline $\mathrm{C}$ & -6.69018 & -5.09432 & -1.41177 \\
\hline $\mathrm{C}$ & -7.7291 & -4.54534 & 0.818113 \\
\hline
\end{tabular}




\begin{tabular}{|c|c|c|c|}
\hline $\mathrm{C}$ & -9.13945 & -4.64077 & -1.24027 \\
\hline $\mathrm{H}$ & -6.68973 & -4.89152 & -2.4944 \\
\hline $\mathrm{H}$ & -5.67962 & -4.88655 & -1.03 \\
\hline $\mathrm{H}$ & -6.89016 & -6.16791 & -1.26394 \\
\hline $\mathrm{H}$ & -8.48076 & -3.94345 & 1.352451 \\
\hline $\mathrm{H}$ & -7.94779 & -5.60973 & 1.001666 \\
\hline $\mathrm{H}$ & -6.74597 & -4.32505 & 1.259577 \\
\hline $\mathrm{H}$ & -9.31592 & -5.71199 & -1.05806 \\
\hline $\mathrm{H}$ & -9.95028 & -4.08212 & -0.74809 \\
\hline $\mathrm{H}$ & -9.21541 & -4.47156 & -2.32542 \\
\hline $\mathrm{C}$ & -9.02841 & 0.447302 & -2.44104 \\
\hline $\mathrm{C}$ & -9.40751 & 1.563742 & -1.44466 \\
\hline $\mathrm{C}$ & -8.3395 & 1.073939 & -3.6723 \\
\hline $\mathrm{C}$ & -10.3165 & -0.24681 & -2.90815 \\
\hline $\mathrm{H}$ & -9.89834 & 1.142537 & -0.55345 \\
\hline $\mathrm{H}$ & -8.52318 & 2.124365 & -1.10661 \\
\hline $\mathrm{H}$ & -10.1021 & 2.279434 & -1.91382 \\
\hline $\mathrm{H}$ & -8.0482 & 0.296963 & -4.3966 \\
\hline $\mathrm{H}$ & -9.02081 & 1.776556 & -4.17887 \\
\hline $\mathrm{H}$ & -7.43251 & 1.630961 & -3.3926 \\
\hline $\mathrm{H}$ & -10.9788 & 0.484985 & -3.39577 \\
\hline $\mathrm{H}$ & -10.1086 & -1.0455 & -3.63681 \\
\hline $\mathrm{H}$ & -10.8716 & -0.68723 & -2.06583 \\
\hline $\mathrm{C}$ & -4.68684 & 3.64353 & 2.885579 \\
\hline $\mathrm{C}$ & -5.19642 & 2.623232 & 3.915735 \\
\hline $\mathrm{C}$ & -3.28156 & 4.119141 & 3.313537 \\
\hline $\mathrm{C}$ & -5.65962 & 4.83959 & 2.878366 \\
\hline $\mathrm{H}$ & -6.18205 & 2.220689 & 3.634701 \\
\hline $\mathrm{H}$ & -4.50204 & 1.780665 & 4.036818 \\
\hline $\mathrm{H}$ & -5.29642 & 3.106015 & 4.899927 \\
\hline $\mathrm{H}$ & -2.89241 & 4.887374 & 2.629087 \\
\hline $\mathrm{H}$ & -3.31087 & 4.54761 & 4.328755 \\
\hline $\mathrm{H}$ & -2.5624 & 3.286354 & 3.30601 \\
\hline $\mathrm{H}$ & -5.75039 & 5.260083 & 3.892428 \\
\hline $\mathrm{H}$ & -5.31804 & 5.649788 & 2.217905 \\
\hline $\mathrm{H}$ & -6.66255 & 4.530361 & 2.544453 \\
\hline $\mathrm{C}$ & -3.99854 & 4.315873 & -2.11352 \\
\hline $\mathrm{C}$ & -2.86379 & 5.32302 & -1.84026 \\
\hline $\mathrm{C}$ & -3.66607 & 3.569605 & -3.41542 \\
\hline $\mathrm{C}$ & -5.32744 & 5.077775 & -2.30878 \\
\hline $\mathrm{H}$ & -3.03638 & 5.905747 & -0.9236 \\
\hline $\mathrm{H}$ & -1.89656 & 4.810069 & -1.74126 \\
\hline $\mathrm{H}$ & -2.78075 & 6.036983 & -2.67499 \\
\hline $\mathrm{H}$ & -4.47644 & 2.888991 & -3.71818 \\
\hline
\end{tabular}




\begin{tabular}{|c|c|c|c|}
\hline $\mathrm{H}$ & -3.52203 & 4.29317 & -4.23236 \\
\hline $\mathrm{H}$ & -2.74004 & 2.981814 & -3.32079 \\
\hline $\mathrm{H}$ & -5.25513 & 5.770824 & -3.16278 \\
\hline $\mathrm{H}$ & -6.1544 & 4.376696 & -2.50419 \\
\hline $\mathrm{H}$ & -5.59009 & 5.664654 & -1.41562 \\
\hline $\mathrm{C}$ & 2.745555 & -5.91089 & 0.043272 \\
\hline $\mathrm{C}$ & 3.581131 & -5.95622 & 1.168847 \\
\hline $\mathrm{C}$ & 3.326261 & -5.12318 & 2.25029 \\
\hline $\mathrm{C}$ & 2.228015 & -4.25548 & 2.205698 \\
\hline $\mathrm{C}$ & 1.373498 & -4.19418 & 1.102468 \\
\hline $\mathrm{C}$ & 1.665223 & -5.03638 & 0.013094 \\
\hline $\mathrm{C}$ & 0.185325 & -3.2974 & 1.162233 \\
\hline $\mathrm{C}$ & -0.04803 & -2.5984 & 2.3175 \\
\hline $\mathrm{C}$ & -1.43962 & -2.3965 & 2.856465 \\
\hline $\mathrm{C}$ & 0.834787 & -2.78889 & 3.485241 \\
\hline $\mathrm{O}$ & 2.000551 & -3.50146 & 3.307204 \\
\hline $\mathrm{O}$ & -2.3894 & -3.13888 & 2.70954 \\
\hline $\mathrm{C}$ & -0.97071 & -3.79214 & 0.305272 \\
\hline $\mathrm{O}$ & 0.598134 & -2.45628 & 4.624539 \\
\hline $\mathrm{O}$ & -1.53305 & -1.25146 & 3.557292 \\
\hline $\mathrm{C}$ & -2.4616 & 0.164898 & 5.216211 \\
\hline $\mathrm{C}$ & -2.72913 & -1.00955 & 4.299173 \\
\hline $\mathrm{H}$ & 2.948012 & -6.55237 & -0.8156 \\
\hline $\mathrm{H}$ & 4.432448 & -6.63981 & 1.195286 \\
\hline $\mathrm{H}$ & 3.945622 & -5.12785 & 3.147199 \\
\hline $\mathrm{H}$ & 1.039356 & -4.99588 & -0.87739 \\
\hline $\mathrm{H}$ & -0.65861 & -3.95949 & -0.73025 \\
\hline $\mathrm{H}$ & -1.33014 & -4.7401 & 0.734996 \\
\hline $\mathrm{H}$ & -1.82401 & -3.11501 & 0.293219 \\
\hline $\mathrm{H}$ & -3.36042 & 0.401853 & 5.805469 \\
\hline $\mathrm{H}$ & -1.63818 & -0.07131 & 5.905806 \\
\hline $\mathrm{H}$ & -2.17888 & 1.05679 & 4.636755 \\
\hline $\mathrm{H}$ & -3.55414 & -0.80325 & 3.593913 \\
\hline $\mathrm{H}$ & -2.99936 & -1.92086 & 4.854106 \\
\hline
\end{tabular}

